\title{
Angle Resolved Photoemission Spectroscopy Studies of the Mott Insulator to Superconductor Evolution in $\mathrm{Ca} 2-\mathrm{xNaxCuO2Cl2}$
}

Kyle Michael Shen

Ph.D. Thesis 


\title{
ANGLE-RESOLVED PHOTOEMISSION SPECTROSCOPY STUDIES OF THE MOTT INSULATOR TO SUPERCONDUCTOR EVOLUTION IN $\mathrm{CA}_{2-X} \mathrm{NA}_{X} \mathrm{CUO}_{2} \mathrm{CL}_{2}$
}

\author{
A DISSERTATION \\ SUBMITTED TO THE DEPARTMENT OF APPLIED PHYSICS \\ AND THE COMMITTEE ON GRADUATE STUDIES \\ OF STANFORD UNIVERSITY \\ IN PARTIAL FULFILLMENT OF THE REQUIREMENTS \\ FOR THE DEGREE OF \\ DOCTOR OF PHILOSOPHY
}

Kyle Michael Shen

May 2005 
(C) Copyright by Kyle Michael Shen 2005

All Rights Reserved 
I certify that I have read this dissertation and that, in my opinion, it is fully adequate in scope and quality as a dissertation for the degree of Doctor of Philosophy.

\section{Zhi-Xun Shen Principal Adviser}

I certify that I have read this dissertation and that, in my opinion, it is fully adequate in scope and quality as a dissertation for the degree of Doctor of Philosophy.

Shoucheng Zhang

I certify that I have read this dissertation and that, in my opinion, it is fully adequate in scope and quality as a dissertation for the degree of Doctor of Philosophy.

\section{Martin Greven}

Approved for the University Committee on Graduate Studies. 


\section{To Mom and Dad}




\section{Everything I needed to know about doing research, I learned from The Simpsons}

Facts are meaningless! You could use facts to prove anything that's even remotely true!

- Homer J. Simpson

Ah, there's nothing more exciting than science. You get all the fun of sitting still, being quiet, writing down numbers, paying attention... Science has it all.

- Principal Seymour Skinner

How is education supposed to make me feel smarter? Besides, every time I learn something new, it pushes some old stuff out of my brain. Remember when I took that home winemaking course, and I forgot how to drive?

- Homer J. Simpson

Well, it is just a prototype. With proper funding, I'm confident this little baby could destroy an area the size of New York City.

- Professor John Frink

The internet wasn't created for mockery. It was supposed to help researchers at different universities share data sets. It was!

- Homer J. Simpson

You tried your best and you failed miserably. The lesson is, never try. - Homer J. Simpson

Well, it appears science has faltered once again in the face of overwhelming religious evidence.

- Rev. Timothy Lovejoy 


\section{Abstract}

It is widely believed that many of the exotic physical properties of the high- $\mathrm{T}_{\mathrm{c}}$ cuprate superconductors arise from the proximity of these materials to the strongly correlated, antiferromagnetic Mott insulating state. Therefore, one of the fundamental questions in the field of high-temperature superconductivity is to understand the insulator-tosuperconductor transition and precisely how the electronic structure of Mott insulator evolves as the first holes are doped into the system. This dissertation presents highresolution, doping dependent angle-resolved photoemission (ARPES) studies of the cuprate superconductor $\mathrm{Ca}_{2-x} \mathrm{Na}_{x} \mathrm{CuO}_{2} \mathrm{Cl}_{2}$, spanning from the undoped parent Mott insulator to a high-temperature superconductor with a $\mathrm{T}_{\mathrm{c}}$ of $22 \mathrm{~K}$. A phenomenological model is proposed to explain how the spectral lineshape, the quasiparticle band dispersion, and the chemical potential all progress with doping in a logical and selfconsistent framework. This model is based on Franck-Condon broadening observed in polaronic systems where strong electron-boson interactions cause the quasiparticle residue, $Z$, to be vanishingly small. Comparisons of the low-lying states to different electronic states in the valence band strongly suggest that the coupling of the photohole to the lattice (i.e. lattice polaron formation) is the dominant broadening mechanism for the lower Hubbard band states.

Combining this polaronic framework with high-resolution ARPES measurements finally provides a resolution to the long-standing controversy over the behavior of the chemical potential in the high- $\mathrm{T}_{\mathrm{c}}$ cuprates. This scenario arises from replacing the conventional Fermi liquid quasiparticle interpretation of the features in the Mott insulator by a Franck-Condon model, allowing the reassignment of the position of the quasiparticle pole. As a function of hole doping, the chemical potential shifts 
smoothly into the valence band while spectral weight is transferred from incoherent weight at high energies to a coherent quasiparticle peak near $\mathrm{E}_{\mathrm{F}}$. The combined shift in the chemical potential and Fermi wavevector, $\mathbf{k}_{\mathrm{F}}$, closely corresponds to a rigid band shift into the faint excitation branch defined by the low-energy quasiparticles.

In addition, the detailed doping evolution of the low-energy excitations has been studied throughout $\mathbf{k}$-space. A large, hole-like contour emerges with doping, although well-defined quasiparticle peaks are only observed along a small arc around the $(0,0)-(\pi, \pi)$ nodal line. Near the $(\pi, 0)$ antinodes, faint parallel segments are found with a nesting wavevector and energy dependence very similar to the $4 a_{0} \times 4 a_{0}$ checkerboard pattern observed by scanning tunneling microscopy. However, these measurements reveal a striking dichotomy between the real and momentum-space probes, where antinodal charge ordering is emphasized in the tunnelling measurements, while ARPES is most sensitive to excitations near the $d$-wave nodal line. These results emphasize the importance of momentum anisotropy in determining the complex electronic properties of the cuprates and places strong constraints on theoretical models of the charge ordered state. 


\section{Acknowledgments}

\section{A long time ago, at a beamline far, far away...}

The beamline wasn't that far away, but seven years likely qualifies as a long time ago. Luckily, the old adage "time flies when you're having fun" certainly rings true in this case. It has been a lot of fun.

First of all, I would like to thank my advisor, Zhi-Xun Shen, for being a terrific boss. Z.-X.'s incredible enthusiasm for doing science, his boundless energy, and his limitless creativity have always been an inspiration to me. Thanks to him, I have led an extremely privileged life as a graduate student : I was able to address some of the most stimulating problems in physics using some of the world's most sophisticated and expensive toys, all while traveling around the world (on his dime) to give talks in Japan, Brazil, Germany, Britain, and Taiwan and interacting with many of the leading physicists in the field.

Even since I was a first-year student, Z.-X. has allowed me a great deal of independence and latitude to pursue my own ideas and projects, while at the same time providing just the right amount of encouragement and advice. Perhaps his most valuable lessons have not been in the niceties of equations that could otherwise be learned from a textbook, but in demonstrating, by example, those subtle intangibles 
necessary to be a world-class scientist. However, the best thing that I can say about Z.-X. is that he cares a great deal about the welfare and success of every one of his group members, both professionally and personally. I owe him an enormous debt of gratitude and feel very fortunate and proud to count myself amongst his (now former!) students.

I entered Z.-X.'s group at a particularly opportune time, with many senior graduate students and postdocs to help mentor me along, and learned a tremendous amount from this talented core group in my first couple years. Given that head start, one would think that it could only be through either sheer incompetence or complete laziness that it would have taken me seven years to graduate (I like to think of it as the subtle interplay of the two). They were not only great to work with and learn from, but I count them all as good friends.

First, I must start by thanking Donghui Lu, who has been the only member to overlap with me for my whole duration in the group. I can think of no better description of Donghui than "Beamline Jesus". He has made the lives of everyone in Z.-X.'s group, particularly mine, so much easier through his selfless dedication and his instant recall of the model, serial number, and life history of every part on the endstation. Andrea Damascelli acted as my surrogate advisor through my first year or two in the group, and the psychological trauma from his mentoring is now starting to slowly heal, thanks to years of counseling and therapy. I inherited the oxychloride project from Filip Ronning, and he really deserves a lot of credit for laying down the foundation upon which the work in this thesis is built up. Plus, he was such a multitalented, easygoing, well-adjusted, and all-around great guy that it just sickened me. Peter Armitage and I shared a mutual fondness for biting sarcasm, schadenfreude, The Onion, and "Herring-in-the-Window". I learned a great deal about condensed matter physics by talking with Pete in my first couple years, and for that I am very grateful. Donglai Feng took me under his wing in my first few months in the group and really got me directly involved in the science, and I certainly benefitted from working with him. Finally, "Crazy Old" Changyoung Kim taught me, amongst many other things, the importance of a good pair of beamline pants.

Among the more current generation of students, I interacted the most with 
Weisheng Lee and "King Non" Meevasana, and it has been a lot of fun to watch them mature as scientists. You couldn't meet two nicer guys, and I must say that they tolerated my "big-brothering" (read : abuse) amazingly well. I just hope that I haven't scarred them for life. Nik Ingle and Felix Baumberger were always fun to talk to - I learned a lot about how to do UHV the right way from Nik, and I had interesting and worthwhile scientific discussions with Felix. Also, Yulin Chen, Norman Mannella, Xingjiang Zhou, and Wanli Yang were always very helpful on my forays to Berkeley to work on the spin chamber.

I have been very fortunate throughout my graduate school career to have benefitted from the interactions, advice, and mentoring of many other scientists in our field. Takashi Imai was my undergraduate thesis adviser and started off my research career, and he always continued to give valued advice and encouragement. One of the highlights of my graduate career was the opportunity to collaborate with Hide Takagi and his group. Certainly, our work on the oxychloride was very successful, but Hide also took an active interest in my own personal career and development as a scientist, for which I am very appreciative. I greatly enjoyed my time as a visitor in his group at the University of Tokyo. Hiroshi Eisaki and Naoto Nagaosa deserve a very special thanks, as well. I have very much appreciated and enjoyed discussions and/or collaborations with : Atsushi Fujimori, Teppei Yoshida, Kiyohisa Tanaka, Akihiro Ino, Igor Mazin, David Singh, Dunghai Lee, Olle Gunnarsson, Takashi Mizokawa, J.C. Séamus Davis, George Sawatzky, Andrei Mishchenko, and Tom Devereaux. Sample growers hold a very dear place in the heart of this photoemitter, and I have been very fortunate to collaborate with many talented sample providers : Lance Miller, Takao Sasagawa, Andy Mackenzie, Naoki Kikugawa, Setsuko Tajima, Yoshiteru Maeno, Satoru Nakatsuji, Yoshinori Tokura, and Yoichi Ando. In particular, the oxychloride project would never have happened without the talent and dedication of Yuhki Kohsaka, and he deserves special thanks along with a great amount of credit for the success of this project.

Behind every freshly minted Ph.D., there is always a cast of unsung heroes. Marilyn Gordon was terrific in her role as "Shen Group Mother", taking care of absolutely everyone and everything for many years. Lily Tsukakoshi stepped right into Marilyn's 
shoes without missing a beat and amazed everyone with her dedication and thoughtfulness. I wouldn't be here without all the help of Paula Perron, who took care of every problem with such devastating effectiveness that there is little doubt who really runs the Applied Physics department. I don't even want to imagine where I would be right now without Marilyn, Lily, and Paula. Also, Mark Gibson, Ann Mueller, Pat Bartz, Jennifer Peck, Larry Candido, and Corrina Peng provided helping hands more times than I can count. I would also like to thank Martin Greven and Shoucheng Zhang, who were not only on my reading committee, but were also very good to talk with throughout my time at Stanford. They, along with Mac Beasley and Mark Brongersma, sacrificed a good chunk of their morning on January 20, 2005, to listen to yet another thesis defense. I would also like to acknowledge the Stanford Graduate Fellowships and the National Science and Engineering Research Council of Canada for providing financial support for my first four years of graduate school.

I have to give thanks to the most important people in my life - my friends and family. In particular, my friends in "da22" - Jamie, Sergio, Charles, Miller, Jeff, and Teller - have really helped to keep me sane (sane being a relative term) over the past seven years. Also, many thanks to (in no particular order) : Jason, Cammy, Darrell, Allan, Jon, Jamie, Derek, Andy, Steve, Sheldon, friends from hockey, and many others. A very very special "thank you" goes to Gladys for all her support, sunny optimism, and encouragement. Finally, thanks to Mom and Dad for EVERYTHING, but particularly for always making me feel like I could do anything that I set my mind to, and then backing me $100 \%$ in whatever path I decided to follow - this thesis is dedicated to you.

OK, we're done!

Stanford, California

Kyle M. Shen

May 28, 2005 


\section{Contents}

Abstract $\quad$ vi

Acknowledgments viii

1 Introduction 1

1.1 The Theory of Everything . . . . . . . . . . . . . . . . 1

1.2 Conventional Superconductivity . . . . . . . . . . . . . . 3

1.3 High-Temperature Superconductivity . . . . . . . . . . . . . . . 7

1.3.1 A Brief History of High- $\mathrm{T}_{\mathrm{c}}$ Superconductivity . . . . . . . . 7

1.3.2 Correlated Electron Physics . . . . . . . . . . . . . . . . . 9

1.3.3 Properties of the High-T $\mathrm{T}_{\mathrm{c}}$ Superconductors . . . . . . . . . . . 14

1.3.4 Key Experimental Results from ARPES . . . . . . . . . . . . 20

2 Photoemission Spectroscopy 23

2.1 General Aspects of Photoemission . . . . . . . . . . . . . . . . . 24

2.2 Energetics and Kinematics of ARPES . . . . . . . . . . . . 26

2.3 Spectral Function Interpretation of Photoemission Spectra . . . . . . 33

2.4 Interpretation and Data Analysis of ARPES Spectra . . . . . . . . 40

2.4.1 EDC and MDC Analysis . . . . . . . . . . . . . . . 40

2.4.2 Matrix Element Effects . . . . . . . . . . . . . . . 44

2.5 Experimental Details of the ARPES System at SSRL Beamline 5-4 . 46

2.5.1 Scienta Electron Analyzer and Experimental Endstation . . . 46

2.5.2 Resolution and Detector Issues . . . . . . . . . . 51

2.5.3 Transformation from angles to k . . . . . . . . . . . 53 
3 Properties of $\mathrm{Ca}_{2-x} \mathrm{Na}_{x} \mathrm{CuO}_{2} \mathrm{Cl}_{2} \quad 56$

3.1 Physical Properties of $\mathrm{Ca}_{2-x} \mathrm{Na}_{x} \mathrm{CuO}_{2} \mathrm{Cl}_{2} \ldots \ldots \ldots$. . . . . . 57

3.2 Preparation and Characterization of $\mathrm{Ca}_{2-x} \mathrm{Na}_{x} \mathrm{CuO}_{2} \mathrm{Cl}_{2}$ Samples . . . 61

4 Spectral Analysis of the Mott Insulating Cuprates and the FranckCondon Model $\quad 65$

4.1 General Electronic Structure . . . . . . . . . . . . . . . 66

4.1.1 ARPES Studies of the Valence Band . . . . . . . . . . . . . 68

4.1.2 ARPES Studies of the Lower Hubbard Band . . . . . . . . . . 72

4.1.3 Failure of the Coherent Quasiparticle Scenario . . . . . . . . 76

4.2 Franck-Condon Model . . . . . . . . . . . . . . . . . . . . 81

5 Evidence for Lattice Polaron Formation in the Undoped Cuprates 89

5.1 Photoemission Studies of Small Polaron Systems . . . . . . . . . . . . 92

5.2 Lattice Polaron Formation in $\mathrm{Ca}_{2} \mathrm{CuO}_{2} \mathrm{Cl}_{2}$. . . . . . . . . . . . 95

5.2.1 Polaron Formation in $\mathrm{O} 2 p_{\pi}$ States . . . . . . . . . . . . . 95

5.3 Temperature Dependence of the Franck-Condon Broadening . . . . . 98

5.3.1 Temperature Dependence of Valence Band States . . . . . . . 98

5.3.2 Temperature Dependence of Ca $3 p$ Core Levels . . . . . . . . 101

5.3.3 Temperature Dependence of Franck-Condon Broadening . . . 103

5.3.4 Possible Extrinsic Broadening Factors . . . . . . . . . . . . . . 105

5.4 Theoretical Studies of Small Polaron Systems . . . . . . . . . . . . 108

5.5 Momentum Dependence of Franck-Condon Broadening . . . . . . . . 112

5.6 Polaron Formation and Hierarchy of Energy Scales . . . . . . . . . . 115

6 Doping Dependence of the Electronic Structure 120

6.1 Determination of the Chemical Potential Shift . . . . . . . . . . . . 122

6.2 Doping Evolution of the Low Energy States . . . . . . . . . . . . . . 127

6.3 Theoretical Interpretations of the Doping Evolution . . . . . . . . . . 132

6.3.1 Doping Dependence of Electron-Boson Coupling . . . . . . . . 132

6.3.2 Possible Relationship to Electron-Electron Correlations . . . . 136

6.4 Doping Dependence of Franck-Condon Broadening for O2p $p_{\pi}$. . . . 139 
7 Evolution of the Fermi Surface in $\mathrm{Ca}_{2-x} \mathrm{Na}_{x} \mathrm{CuO}_{2} \mathrm{Cl}_{2}$

7.1 Low-Lying Spectral Weight in $\mathrm{Ca}_{2-x} \mathrm{Na}_{x} \mathrm{CuO}_{2} \mathrm{Cl}_{2} \ldots \ldots \ldots \ldots$

7.2 Determining the "Fermi Surface" in $\mathrm{Ca}_{2-x} \mathrm{Na}_{x} \mathrm{CuO}_{2} \mathrm{Cl}_{2} \ldots \ldots$.

7.2.1 Methodology for Quantifying the Fermi Surface . . . . . . . 153

7.2.2 Application of MDC Methodology to $\mathrm{Ca}_{2-x} \mathrm{Na}_{x} \mathrm{CuO}_{2} \mathrm{Cl}_{2} \ldots 156$

7.3 Charge Ordering in $\mathrm{Ca}_{2-x} \mathrm{Na}_{x} \mathrm{CuO}_{2} \mathrm{Cl}_{2} \ldots \ldots \ldots \ldots$

7.3.1 Evidence from STM of Charge Ordering . . . . . . . . . 158

7.3.2 Evidence for Charge Ordering from ARPES . . . . . . . . 160

7.3.3 Comparison with Other Charge-Ordered Systems Studied by ARPES . . . . . . . . . . . . . . . . 165

7.3.4 Luttinger Volumes in $\mathrm{Ca}_{2-x} \mathrm{Na}_{x} \mathrm{CuO}_{2} \mathrm{Cl}_{2} \ldots \ldots \ldots \ldots$

8 Conclusions and Future Prospects $\quad 175$

8.1 Conclusions . . . . . . . . . . . . . . . . . 175

8.2 Future Directions . . . . . . . . . . . . . . . . 177

A Fully Gapped Excitations in the Lightly Doped Cuprates $\quad 180$

A.1 Background . . . . . . . . . . . . . . . . . . 180

A.2 Experimental Details and Results . . . . . . . . . . . . 182

A.3 Discussion and Implications of Experimental Results . . . . . . . 186

B Anomalous Photon Energy Dependence of ARPES Spectra from $\begin{array}{lr}\mathrm{Ca}_{2-x} \mathrm{Na}_{x} \mathrm{CuO}_{2} \mathrm{Cl}_{2} & 190\end{array}$

B.1 Background . . . . . . . . . . . . . . . . . 190

B.2 Experimental Details and Results . . . . . . . . . . . . . 191

B.3 Discussion of Experimental Results . . . . . . . . . . . . 196

C Surface Electronic Structure of $\mathrm{Sr}_{2} \mathrm{RuO}_{4} \quad 200$

C.1 Background . . . . . . . . . . . . . . . . . . . . 200

C.2 Experimental Details and Results . . . . . . . . . . . . 202

C.3 Discussion and Comparison with Theory . . . . . . . . . 206 
D Electronic Structure of the high-temperature BCS superconductor $\begin{array}{ll}\mathrm{MgB}_{2} & \mathbf{2 1 0}\end{array}$

D.1 Background . . . . . . . . . . . . . . . . . 210

D.2 Experimental Details and Results . . . . . . . . . . . . . 211

D.3 Discussion . . . . . . . . . . . . . . . . . . . . . 217

$\begin{array}{ll}\text { Bibliography } & 219\end{array}$ 


\section{List of Tables}

3.1 Lattice Constants of $\mathrm{Ca}_{2} \mathrm{CuO}_{2} \mathrm{Cl}_{2} \ldots \ldots \ldots \ldots$ 


\section{List of Figures}

1.1 Illustration of the Meissner Effect . . . . . . . . . . . . . 4

1.2 Cooper Pairing in a BCS Superconductor . . . . . . . . . . . 5

1.3 Periodic Table of Superconductors . . . . . . . . . . . . . . . . 6

1.4 Highest $\mathrm{T}_{\mathrm{c}}$ Superconductor Discovered as a Function of Time . . . . 7

1.5 Charge Reservoir Layers in the Cuprates . . . . . . . . . . . . . . 9

1.6 Conceptualization of Landau Quasiparticles . . . . . . . . . . . 10

1.7 Schematic of a Zhang-Rice Singlet . . . . . . . . . . . . . . . 12

1.8 Generic Cuprate Phase Diagram . . . . . . . . . . . . . . . . . 15

1.9 Resistivity of $\mathrm{La}_{2-x} \mathrm{Sr}_{x} \mathrm{CuO}_{4} \ldots \ldots \ldots \ldots \ldots \ldots$

1.10 Optical Conductivity Results from $\mathrm{La}_{2-x} \mathrm{Sr}_{x} \mathrm{CuO}_{4} \ldots \ldots \ldots$

1.11 Phase Sensitive Measurements of the $d$-wave Order Parameter . . . 19

1.12 Shifting and Pinned Chemical Potentials . . . . . . . . . . . 21

2.1 Improvements in the Energy Resolution of Photoemission . . . . . . 25

2.2 Energetics of Photoemission Process _ . . . . . . . . . . 27

2.3 Kinematics of Photoemission Process . . . . . . . . . . . . 28

2.4 The Three-Step Model . . . . . . . . . . . . . . . . . . . . . . . 31

2.5 Photoelectron "Universal Curve" . . . . . . . . . . . . . . . . . . . 32

2.6 Interactions and ARPES $\ldots \ldots \ldots \ldots \ldots \ldots$

2.7 Simulation of $\mathcal{A}(\mathbf{k}, \omega) \ldots \ldots \ldots \ldots \ldots$

2.8 Illustration of EDCs and MDCs . . . . . . . . . . . . . 41

2.9 Electron-Phonon Interactions in $\operatorname{Be}(0001) \quad \ldots \ldots \ldots \ldots \ldots$

2.10 Two-Pole Treatment of Electron-Phonon Interaction . . . . . . . . . 43

2.11 Photoelectron Matrix Elements . . . . . . . . . . . . . . 44 
2.12 SSRL Beamline $5-4 \ldots \ldots \ldots \ldots \ldots \ldots \ldots$

2.13 Schematic of Scienta Analyzer on Beamline . . . . . . . . . . 48

$2.14200 \mathrm{~mm}$ Gammadata Scienta Electron Analyzers . . . . . . . . . . . 49

2.15 Demonstration of Angle-Mode Improvements . . . . . . . . . . 50

2.16 Improvements in Data Throughput and Collection Efficiency . . . . 50

2.17 Low Temperature Sample Manipulator . . . . . . . . . . . . . 51

2.18 Angle-to-Momentum Conversion . . . . . . . . . . . . . . . . 54

3.1 Structure and Growth of $\mathrm{Ca}_{2-x} \mathrm{Na}_{x} \mathrm{CuO}_{2} \mathrm{Cl}_{2} \ldots \ldots \ldots \ldots$

3.2 Lattice Parameters and Superconductivity in $\mathrm{Ca}_{2-x} \mathrm{Na}_{x} \mathrm{CuO}_{2} \mathrm{Cl}_{2} \ldots \quad$. 59

3.3 Transport and Optical Measurements of $\mathrm{Ca}_{2-x} \mathrm{Na}_{x} \mathrm{CuO}_{2} \mathrm{Cl}_{2} \ldots \ldots .60$

$3.4 \mu$-SR Measurements of $\mathrm{Ca}_{2-x} \mathrm{Na}_{x} \mathrm{CuO}_{2} \mathrm{Cl}_{2} \ldots \ldots \ldots \ldots \ldots$

3.5 Laue Measurements of $\mathrm{Sr}_{2} \mathrm{CuO}_{2} \mathrm{Cl}_{2}, \mathrm{Ca}_{2-x} \mathrm{Na}_{x} \mathrm{CuO}_{2} \mathrm{Cl}_{2}$, and $\mathrm{Sr}_{2} \mathrm{RuO}_{4} \quad 62$

3.6 LEED Measurements of $\mathrm{Ca}_{2-x} \mathrm{Na}_{x} \mathrm{CuO}_{2} \mathrm{Cl}_{2} \ldots \ldots \ldots$

4.1 Calculated Band Structure and DOS of $\mathrm{Ca}_{2} \mathrm{CuO}_{2} \mathrm{Cl}_{2} \ldots \ldots \ldots 67$

4.2 Crystal Field Splitting and Bonding of $\mathrm{Cu}$ and $\mathrm{O} \ldots \ldots$

4.3 Valence Band of $\mathrm{Ca}_{2} \mathrm{CuO}_{2} \mathrm{Cl}_{2} \ldots \ldots \ldots \ldots \ldots$

4.4 Different Valence Band Types in $\mathrm{Ca}_{2} \mathrm{CuO}_{2} \mathrm{Cl}_{2} \ldots \ldots \ldots \ldots \ldots$

4.5 EDCs of $\mathrm{Ca}_{2} \mathrm{CuO}_{2} \mathrm{Cl}_{2}$ Taken Along High Symmetry Lines . . . . . . 73

4.6 Dispersion along High Symmetry Lines: Experiment and Theory . . . 74

4.7 Fits to $\mathrm{Ca}_{2} \mathrm{CuO}_{2} \mathrm{Cl}_{2}$ and Comparison with $\mathrm{Sr}_{2} \mathrm{RuO}_{4} \ldots \ldots \ldots$

4.8 Evolution of $\mu$ and Spectral Weight with Doping . . . . . . . . . . 80

4.9 Illustration of the Franck Condon Principle . . . . . . . . . . . 83

4.10 Results from the Independent Electron-Boson Model . . . . . . . . 88

5.1 Cartoon Schematic of Lattice Polaron Formation _ . . . . . . . . 90

5.2 Core Level Spectroscopy of Alkali Halides . . . . . . . . . . . . . . 93

5.3 Franck-Condon Broadening of $\mathrm{O} 2 p_{\pi}$ States . . . . . . . . . . 96

5.4 Temperature Dependence of LHB States . . . . . . . . . . . . . 99

5.5 Temperature Dependence of $\mathrm{O} 2 p_{\pi}$ States . . . . . . . . . . 100

5.6 Temperature Dependence of Ca $3 p$ Core Levels . . . . . . . . . . . . . 102 
5.7 Temperature Dependence of Franck-Condon Broadening . . . . . . . 104

5.8 Quantum Monte Carlo Calculations for the $t-J+$ Phonon Model . . . 110

5.9 Calculations of Electron-Phonon Coupling on the Spectral Function . 111

5.10 Momentum Dependence of Franck-Condon Broadening . . . . . . . . 113

5.11 Theoretical Momentum Dependence of LHB Width . . . . . . . . . . 115

5.12 Evidence for the Incoherent High-Energy $t$ Branch . . . . . . . . . . . 116

5.13 Schematic of the Effects of $t, J$, and Phonon Effects on ARPES . . . 118

6.1 Valence Band EDC Stacks for $\mathrm{Ca}_{2-x} \mathrm{Na}_{x} \mathrm{CuO}_{2} \mathrm{Cl}_{2}$. . . . . . . . . . . 123

6.2 Oxygen Valence Bands for $\mathrm{Ca}_{2-x} \mathrm{Na}_{x} \mathrm{CuO}_{2} \mathrm{Cl}_{2}$. . . . . . . . . . . . . 124

6.3 Shift of Chemical Potential from Various Cuprates . . . . . . . . . . . 126

6.4 Proposed Model for Density of States . . . . . . . . . . . . . . . . . . 128

6.5 Scenarios for Doping a Band Insulator . . . . . . . . . . . . . . . . . 130

6.6 Doping Dependence of Nodal States: EDCs . . . . . . . . . . . . . . 131

6.7 Doping Dependence of Low Energy States . . . . . . . . . . . . . . 133

6.8 Results from Dynamical Mean Field Theory . . . . . . . . . . . . . . 137

6.9 Doping Dependence of $\mathrm{O} 2 p_{\pi} \mathrm{FWHM}$. . . . . . . . . . . . . . . . . . 140

6.10 Electron-phonon Coupling from O2p $p_{\pi}$ FWHM . . . . . . . . . . . . 144

7.1 Pictorials of Fermi Surface Evolution in Doped Mott Insulator . . . . 147

7.2 Possibilities for Fermi Surface Evolution . . . . . . . . . . . . . . 148

7.3 Fermi Surface Intensity Maps for $\mathrm{Ca}_{2-x} \mathrm{Na}_{x} \mathrm{CuO}_{2} \mathrm{Cl}_{2}$. . . . . . . . . 150

7.4 "Bird's Eye View" of Near-E $E_{\mathrm{F}}$ Spectral Intensity . . . . . . . . . . . . 151

7.5 Angular Distribution of Spectral Weight . . . . . . . . . . . . . . . 152

7.6 EDCs Along the $\mathrm{Ca}_{2-x} \mathrm{Na}_{x} \mathrm{CuO}_{2} \mathrm{Cl}_{2}$ FS . . . . . . . . . . . . . 153

7.7 Application of MDC $\mathbf{k}_{\mathrm{F}}$ Determination Method . . . . . . . . . . . . 154

7.8 Extracted $\mathrm{k}_{\mathrm{F}} \mathrm{S}$ from $\mathrm{Ca}_{1.9} \mathrm{Na}_{0.10} \mathrm{CuO}_{2} \mathrm{Cl}_{2} \ldots \ldots \ldots \ldots$

7.9 Extracted $\mathbf{k}_{\mathrm{F}} \mathrm{s}$ from $\mathrm{Ca}_{2-x} \mathrm{Na}_{x} \mathrm{CuO}_{2} \mathrm{Cl}_{2}$. . . . . . . . . . . . . 158

7.10 Comparison of $\mathbf{k}_{\mathrm{F}}$ with Tight-Binding Fits . . . . . . . . . . . . . . . 159

7.11 STM Constant-Current Images of $\mathrm{Ca}_{2-x} \mathrm{Na}_{x} \mathrm{CuO}_{2} \mathrm{Cl}_{2}$. . . . . . . . 160

$7.124 a_{0} \times 4 a_{0}$ Charge-Ordering observed by STM . . . . . . . . . . . 161

7.13 ARPES Spectral Maps versus STS $d I / d V$ maps . . . . . . . . . . . . 162 
7.14 Nodal and Antinodal MDCs . . . . . . . . . . . . . . . . 163

7.15 Comparison of Fermi Surfaces with Luttinger Sum Rule . . . . . . . 168

7.16 Minimum Luttinger Volume for $x=0.10 \ldots \ldots \ldots$

7.17 Spectral Maps from Cluster Dynamical Mean-Field Theory . . . . . . 171

7.18 Comparison between $\mathrm{La}_{2-x} \mathrm{Sr}_{x} \mathrm{CuO}_{4}$ and $\mathrm{Ca}_{2-x} \mathrm{Na}_{x} \mathrm{CuO}_{2} \mathrm{Cl}_{2} \ldots \ldots$

A.1 EDCs of $\mathrm{Ca}_{1.9} \mathrm{Na}_{0.10} \mathrm{CuO}_{2} \mathrm{Cl}_{2}$ With a Small Leading-Edge Gap . . . 183

A.2 Summary of LEGs from Different Samples . . . . . . . . . . . . 184

A.3 Summary of Leading Edge Gaps . . . . . . . . . . . . . . . 187

B.1 Image Plots Showing Photon Energy Dependence of Spectra . . . . 192

B.2 Photon Energy Dependence of EDC Lineshapes _ . . . . . . . . . 195

B.3 Coherent and Incoherent Weight versus Doping and Photon Energy . 197

C.1 Fermi Surfaces of Bulk and Surface $\mathrm{Sr}_{2} \mathrm{RuO}_{4} \ldots \ldots \ldots \ldots$

C.2 EDCs and MDCs from $\mathrm{Sr}_{2} \mathrm{RuO}_{4} \ldots \ldots \ldots \ldots \ldots \ldots$

C.3 Data from $\mathrm{Sr}_{2} \mathrm{RuO}_{4}$ Surface and Bulk-Derived States near X . . . . 205

C.4 Calculations for Magnetic and Nonmagnetic $\mathrm{Sr}_{2} \mathrm{RuO}_{4}$ Surfaces . . . 208

D.1 EDCs of $\mathrm{MgB}_{2}$ Along High-Symmetry Directions . . . . . . . . 213

D.2 Second-Derivative Plots of $\mathrm{MgB}_{2} \quad \ldots \ldots \ldots \ldots \ldots \ldots$

D.3 Surface State of $\mathrm{MgB}_{2} \ldots \ldots \ldots \ldots \ldots \ldots$ 


\section{Chapter 1}

\section{Introduction}

\subsection{The Theory of Everything}

The beautiful paradox of solid state physics is that it is a field which is always rife with new surprises and controversies, yet the microscopic interactions which govern all behavior have been known for the past eighty years, and can be written down on a cocktail napkin by an undergraduate physics student. This "master equation" is simply the non-relativistic Schrodinger's equation for electrons and nuclei interacting via the Coulomb interaction, shown as Equation 1.1

$\mathcal{H}=-\sum_{j}^{N_{e}} \frac{\hbar^{2}}{2 m} \nabla_{j}^{2}-\sum_{\alpha}^{N_{i}} \frac{\hbar^{2}}{2 M_{\alpha}} \nabla_{\alpha}^{2}-\sum_{j}^{N_{e}} \sum_{\alpha}^{N_{i}} \frac{Z_{\alpha} e^{2}}{\left|\vec{r}_{j}-\vec{R}_{\alpha}\right|}+\sum_{j \ll k}^{N_{e}} \frac{e^{2}}{\left|\vec{r}_{j}-\vec{r}_{k}\right|}+\sum_{\alpha \ll \beta}^{N_{j}} \frac{Z_{\alpha} Z_{\beta} e^{2}}{\left|\vec{R}_{\alpha}-\vec{R}_{\beta}\right|}$

where $\hbar$ is Planck's constant, $e$ and $m$ are the charge and mass of the electron, $r_{j}$ is the location of the $j^{\text {th }}$ electron, and $Z \alpha$ and $M \alpha$ are the atomic number and mass of the $\alpha^{\text {th }}$ nucleus at position $R \alpha$. This so-called "theory of everything" (as coined by Laughlin and Pines [1]) describes virtually everything we see around us, from human beings to high-temperature superconductivity, and certainly encapsulates all of chemistry, biology, and solid-state physics. Only a few things that we encounter in our day-to-day lives are not described by this Hamiltonian: the sun and stars 
(thermonuclear fusion) or apples falling to the ground (gravity). The question, then, is why do people continue to study seemingly "solved" topics (such as high-temperature superconductivity or organic chemistry) when the basic Hamiltonian determining all the underlying physics is already well known?

The answer is that Equation 1.1 cannot be solved analytically for any system beyond a two-particle system (such as the hydrogen atom), and cannot be solved numerically for anything beyond a handful of particles $(N>10)$ with even the most powerful computers in existence (or likely will ever come into existence). Seeing as how even a single silicon atom has 14 electrons, it becomes immediately obvious that attempting to understand the properties of large molecules, let alone entire crystals, with a brute force, first-principles approach with Equation 1.1, is utterly futile. Knowledge of the underlying Hamiltonian may provide little or no insight or predictive power into the important physical properties of the system in question. The good news is that the insolubility of Equation 1.1 certainly guarantees employment for future generations of solid state physicists, chemists, and biologists. Despite the general insolubility and complexity of Equation 1.1, Nature is not a hopelessly complicated mess. Beautiful and elegant laws and phenomena can emerge with higher organizing principles than can be robust against changes in the nitty-gritty details of the starting constituents. To take the example of condensed matter physics, some of the best-known "emergent states" include crystallinity, Landau Fermi liquid theory, superconductivity, magnetism, superfluidity, and quantum Hall states. Discovering and understanding such new states of matter remains at the heart of the quantum many-body problem in physics.

One can often attempt to reduce the complexity of the Hamiltonian in Equation 1.1 to a more easily solved model, in the hopes that this simpler model, while incomplete, may still capture the essential properties in question. For instance, while even attempting an exact numerical solution for a single $\mathrm{Cu}$ atom is essentially impossible, rather simple models are able to provide an excellent description of the relevant properties of crystalline $\mathrm{Cu}$ with $10^{23} \mathrm{Cu}$ atoms. Simple "one-electron band theories" have provided a remarkably accurate description of a wide range of materials. In such models, one effectively neglects the electron-electron interactions and 
the interaction between nuclei, and reduces the problem of $10^{24}$ interacting electrons to a bare model of a single electron propagating through a static periodic potential defined by the crystal lattice. Such a concept dates back to the work of Bloch and Wilson in the 1930's, before it was even clear why such a simple approach should be so successful. Today, band theory remains alive and well, and is the basis of the semiconductor industry which in turn is the foundation for virtually all of high-technology today. Despite the success of the one-electron picture in explaining the properties of a wide number of compounds, it should not be terribly surprising that there can be instances where band theory not only fails, but fails spectacularly. The motivation behind this dissertation is to investigate such novel quantum states of matter which arise as a result of strong quantum many-body interactions - the Mott insulator and the high-temperature superconductor being two of the most prominent examples of such states.

\subsection{Conventional Superconductivity}

Superconductivity was discovered by Heike Kammerlingh Onnes in elemental mercury in 1911 [2], only three years after his successful liquefaction of helium, and was awarded the Nobel Prize in Physics shortly thereafter in 1913. At the time, little was known about the effect, other than it manifested itself as an abrupt drop of the electrical resistivity to absolutely zero (such that a persistent current set up in a superconducting material should not dissipate in any less than $10^{5}$ years!), and that it was observed in a number of elemental metals such as tin and lead. The next hallmark of superconductivity was discovered by Meissner and Ochsenfeld in 1933 [3] was perfect diamagnetism - the complete expulsion of a magnetic field from the interior of a superconductor, now known as the Meissner effect, shown in Figure 1.1.

However, to this point, there was very little theoretical understanding of the mechanism of superconductivity. This changed in the 1930's with the work of the London brothers who introduced a phenomenological theory of the electrodynamics of superconductors [4], and later in the 1950's by the work of Ginzburg and Landau in applying their powerful theory of second-order phase transitions to superconductivity 


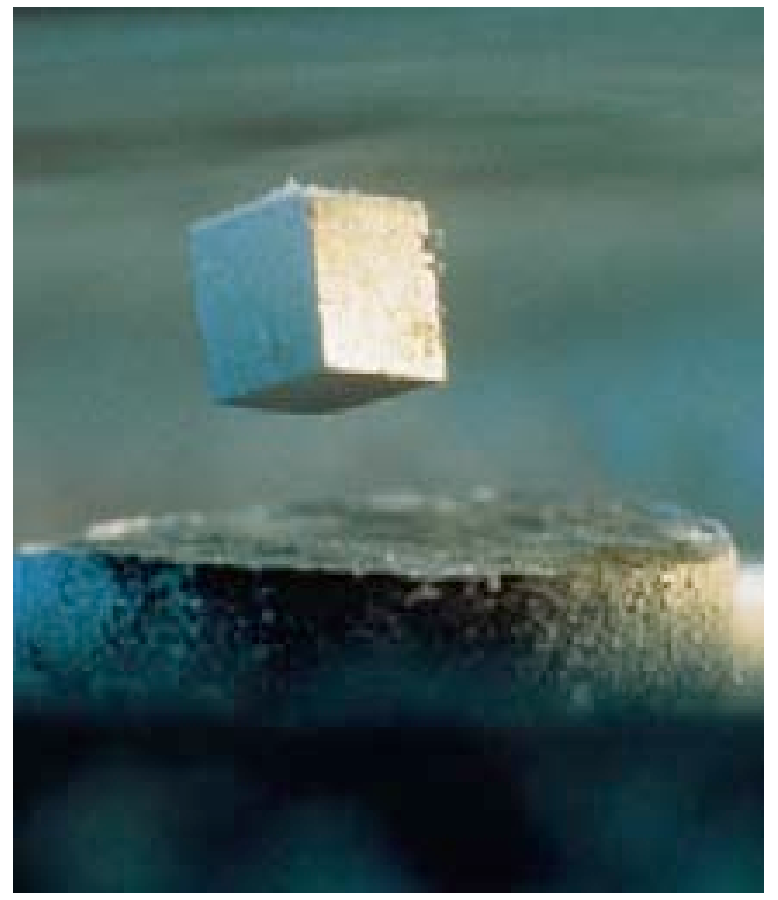

Figure 1.1: Demonstration of the Meissner effect (perfect diamagnetism) in a high- $\mathrm{T}_{\mathrm{c}}$ superconductor. The force provided by the expulsion of the permanent magnet's field from the interior of the superconductor counteracts gravity and results in levitation.

[5]. However, a microscopic theory of superconductivity remained elusive until the breakthrough by Bardeen, Cooper, and Schrieffer and the development of the BCS theory of superconductivity [6], for which they won the Nobel Prize in 1972. They explained superconductivity to arise from the pairing of individual electrons into composite bosons via an attractive effective retarded interaction mediated by the lattice, and the the subsequent condensation of these bosons into the superfluid. The main problem in understanding how electrons could form bosonic pairs was that electrons should repel each other through the Coulomb interaction. This was addressed by the BCS theory, which explained that the electrons were paired through an intermediary, being the lattice, shown in Figure 1.2. As one electron propagates through the lattice, it causes a local positive polarization of the lattice. However, the electron moves much more quickly than the lattice, so the original electron will have moved far away, before the lattice polarization can relax back to its equilibrium position. This positive 


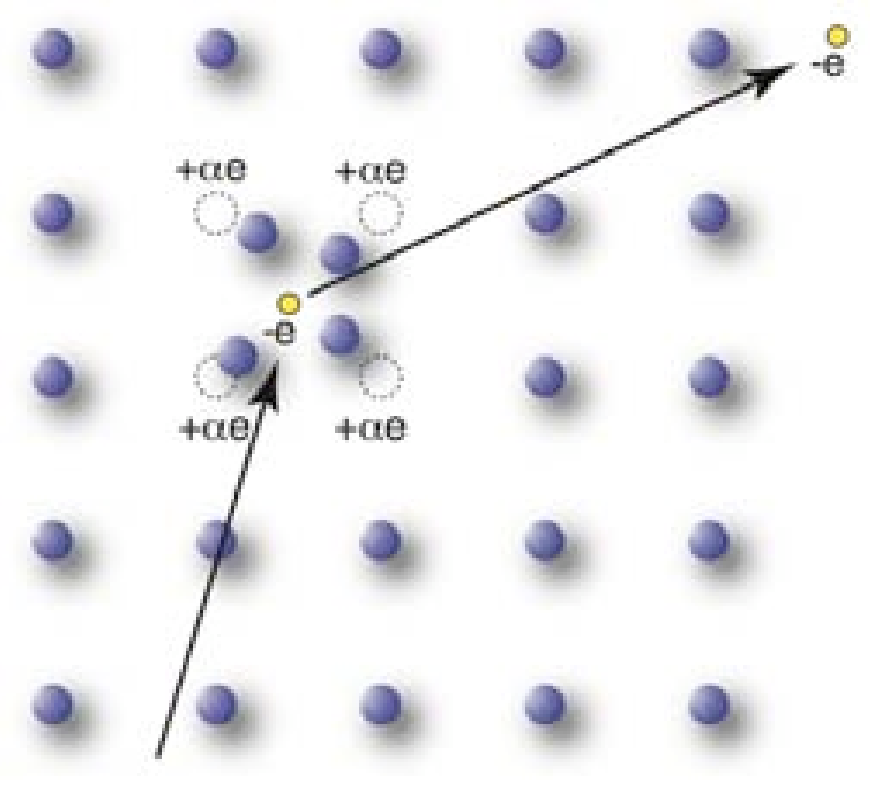

Figure 1.2: Schematic of the retarded effective pairing interaction between electrons in a Cooper pair mediated by the lattice.

polarization can then attract a second electron, resulting in an effective pairing of the first and second electron into a boson. The BCS ground state wavefunction can be expressed as follows

$$
\left|\psi_{B C S}\right\rangle=\prod_{\mathbf{k}}\left(u_{\mathbf{k}}+v_{\mathbf{k}} c_{\mathbf{k} \uparrow}^{\dagger} c_{-\mathbf{k} \downarrow}^{\dagger}\right)\left|\phi_{0}\right\rangle
$$

where $\left|u_{\mathbf{k}}\right|^{2}+\left|v_{\mathbf{k}}\right|^{2}=1, u_{\mathbf{k}}$ and $v_{\mathbf{k}}$ are complex numbers, and $\left|\phi_{0}\right\rangle$ is the vacuum state. There is an order parameter associated with the superconducting state

$$
\Delta e^{i \phi}
$$

where $\Delta$ is the magnitude of the order parameter and the superconducting energy gap, i.e. the energy cost needed to break apart the composite boson or Cooper pair into individual electrons. $\phi$ represents the phase of the superfluid, and macroscopic phase coherence is one of the unique and distinguishing characteristics of the superconducting state from the band metal. Equation 1.2 should also make clear that the superconducting state is entirely different than a perfect conductor, since 

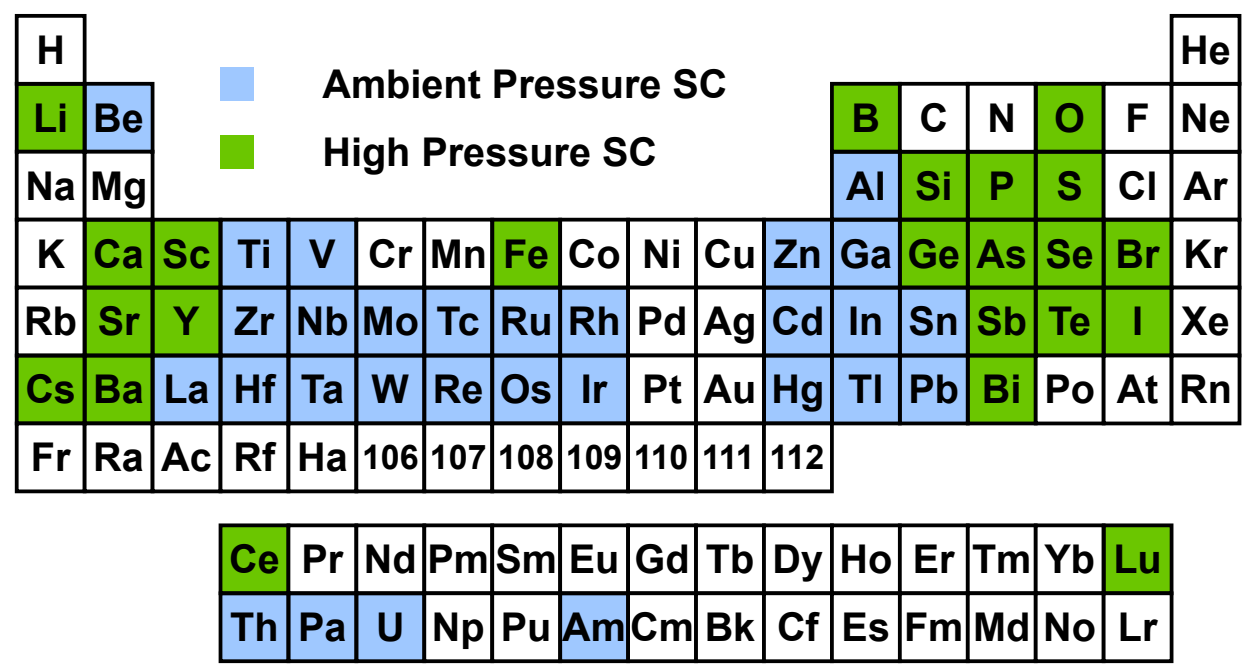

Figure 1.3: Periodic table showing elemental superconductors, including those which superconduct only under high pressures.

the ground state is orthogonal to the Fermi liquid. For instance, a Fermi gas or any perfect crystal will have zero resistance at $\mathrm{T}=0$. However, in any real system, the existence of some minute concentration of defects will exist simply due to entropy considerations. On the other hand, in a superconductor, the transport of electrons is insensitive to the inevitable presence of defects. Today, many superconductors have been discovered. In fact, since the basic ingredients for superconductivity are found in every metal (phonons and conduction electrons), it should not be surprising that superconductivity manifests itself in a wide variety of materials. A periodic table of known elemental superconductors is shown in Figure 1.3.

While many materials exhibit a superconducting ground state, the temperatures at which they superconduct are typically below $\sim 15 \mathrm{~K}$. It was generally believed that due to competing effects (such as charge-density-wave formation or structural instabilities), the maximum superconducting transition temperature for an electronphonon mechanism was on the order of 10-20 K. Today, we known that this is not true, since there are obvious examples of BCS superconductivity with $\mathrm{T}_{\mathrm{c}} \mathrm{s}$ of up to $39 \mathrm{~K}$, such as $\mathrm{MgB}_{2}[7]$. 


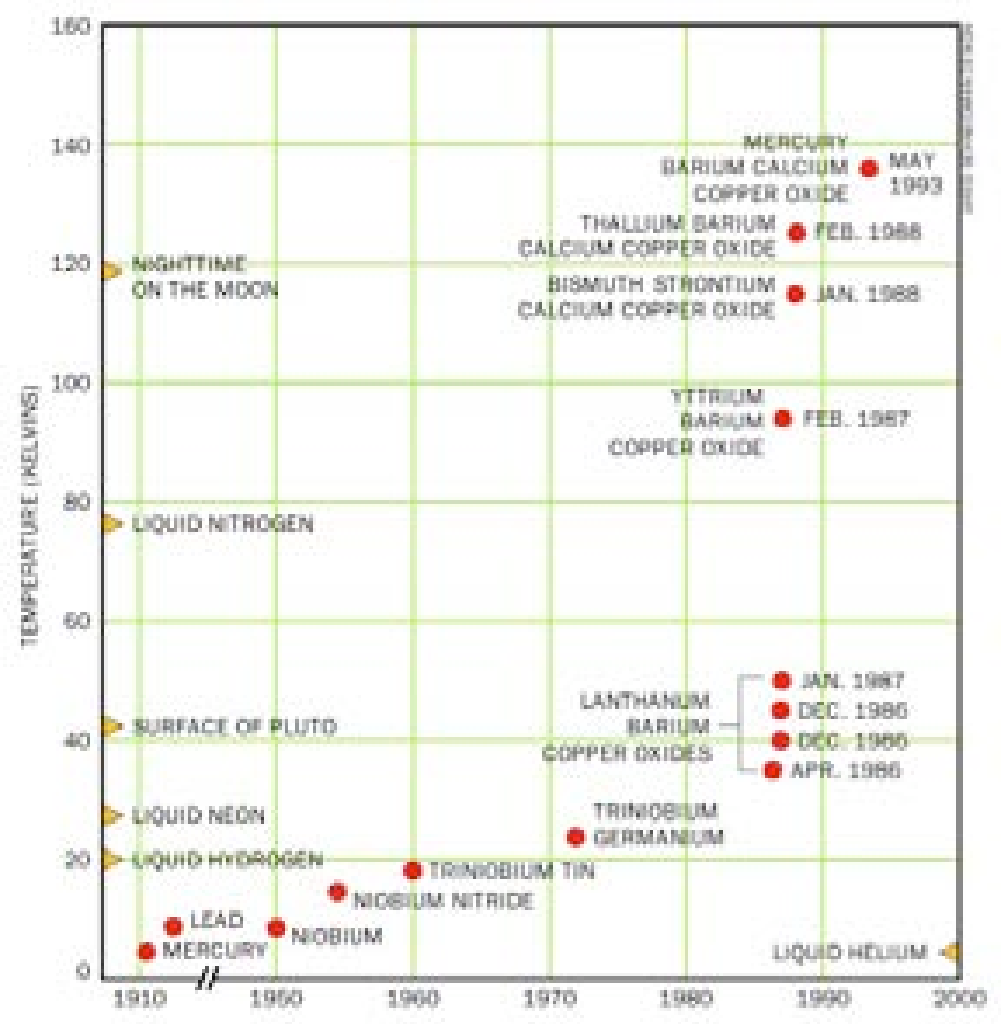

Figure 1.4: Plot showing highest $\mathrm{T}_{\mathrm{c}}$ superconductor discovered as a function of time.

\subsection{High-Temperature Superconductivity}

\subsubsection{A Brief History of High- $\mathrm{T}_{\mathrm{c}}$ Superconductivity}

The field of superconductivity changed dramatically in 1986 with a report of superconductivity at $35 \mathrm{~K}$ in $\mathrm{La}_{2-x} \mathrm{Ba}_{x} \mathrm{CuO}_{4+\delta}$ by Bednorz and Mueller [8]. An enormous burst of activity followed (now often referred to as high- $T_{\mathrm{c}}$ fever), resulting in the discovery of superconductivity at $90 \mathrm{~K}$ in the $\mathrm{YBa}_{2} \mathrm{Cu}_{3} \mathrm{O}_{7-\delta}$ family by $\mathrm{Wu}$ and $\mathrm{Chu}$ just one year later [9]. The ubiquitous plot of " $\mathrm{T}_{\mathrm{c}}$ vs. time" is shown in Figure 1.4.

Over the course of a mere 2 years, $\mathrm{T}_{\mathrm{c}} \mathrm{s}$ skyrocketed up past a balmy $120 \mathrm{~K}$. Currently, the highest $\mathrm{T}_{\mathrm{c}}$ is over $160 \mathrm{~K}$, but this occurs only under the application of high pressure. The first families of high- $\mathrm{T}_{\mathrm{c}}$ compounds discovered were hole-doped compounds, but in 1989, Tokura, Takagi, and Uchida discovered the first class of 
electron-doped high- $\mathrm{T}_{\mathrm{c}}$ superconductors, $\mathrm{Nd}_{2-x} \mathrm{Ce}_{x} \mathrm{CuO}_{4}$ [10]. It was determined that the main constituent of the cuprates was a square $\mathrm{CuO}_{2}$ plane which, when undoped, has one electron per $\mathrm{Cu}$ atom, but was insulating due to strong electronelectron repulsion. Carrier doping occurs either by adding or removing oxygen atoms (typically interstitially) or by cation substitution, both of which take place away from the $\mathrm{CuO}_{2}$ plane, in the so-called "blocking" or "charge reservoir" layers, similar to modulation doping in semiconductor, as shown in Figure 1.5. In fact, these "blocking layers" were for the most part electrically inactive and virtually all charge conduction was found to take place within the $\mathrm{CuO}_{2}$ planes. For typical cuprates, the in-plane to out-of-plane resistivity anisotropy, $\rho_{c} / \rho_{a b}$ is typically on the order of $10^{3}$ to $10^{4}$, implying that the electrons are essentially confined to move solely within the $\mathrm{CuO}_{2}$ plane. As a result, the high- $\mathrm{T}_{\mathrm{c}}$ cuprates are often idealized as a single square $\mathrm{CuO}_{2}$ sheet, since it is generally believed that the single $\mathrm{CuO}_{2}$ plane contains all of the essential physics in the cuprates. A further simplification of this problem is to ignore the details of the individual copper and oxygen orbitals and reduce the problem to a single 2D square grid with zero, one, or two spin- $1 / 2$ fermions per site. While this may capture many aspects of the high- $\mathrm{T}_{\mathrm{C}} \mathrm{s}$, we know this cannot encompass all of the important features of the cuprates. In particular, the high- $T_{c}$ cuprates have optimal $\mathrm{T}_{\mathrm{c}} \mathrm{s}$ which can vary by up to a factor of 5 , so there are clearly chemical and structural effects beyond the idealized $\mathrm{CuO}_{2}$ plane which can dramatically effect the physical properties.

The high transition temperatures observed in the cuprates immediately suggested that a pairing mechanism other than the conventional electron-phonon interaction was responsible for superconductivity. This notion was supported by the fact that the parent material of the cuprates is an antiferromagnetic Mott insulator driven by strong electron-electron correlations. Over the past two decades, a wide array of experimental probes have uncovered some very unusual behaviors in the cuprates, as will be discussed later in this chapter. 


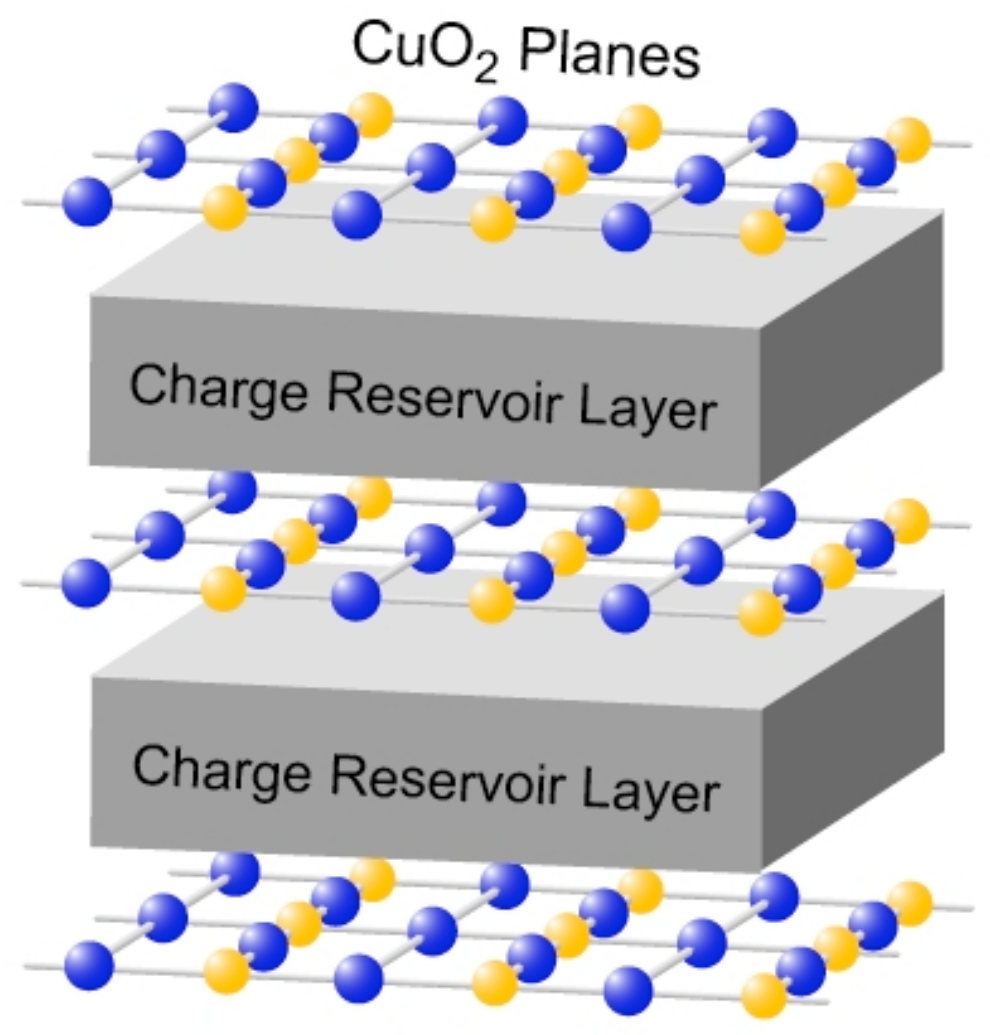

Figure 1.5: Figure showing the $\mathrm{CuO}_{2}$ plane and the charge reservoir layer where chemical doping takes place.

\subsubsection{Correlated Electron Physics}

Given that all real materials possess rather sizable electron-electron correlations, it is rather remarkable that one-electron band theory works so well for such a wide class of materials. Although band theory originated with Bloch in 1928 [11], it was not until Landau's description of quasiparticles and the adiabatic continuity of the quasiparticle liquid to the non-interacting Fermi gas, that one understood exactly why band theory worked as well as it did. Today, the quasiparticle concept is typically referred to as "Fermi liquid" theory, or "Landau Fermi liquid quasiparticles". To be specific, the quasiparticle concept is more general than Fermi liquid theory, which typically addresses only the effects of electron-electron scattering. On the other hand, Landau's general quasiparticle concept can be explained rather intuitively in the sense that 


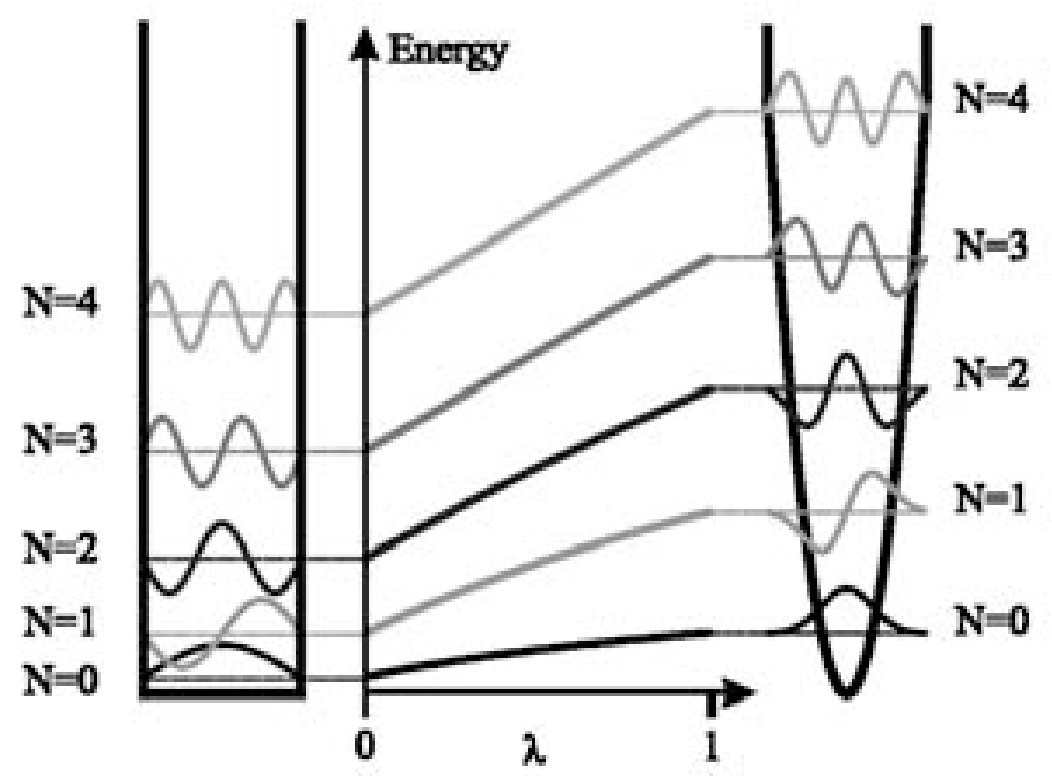

Figure 1.6: A simple conceptualization of the Landau quasiparticle using the eigenstates of the square and parabolic potential wells, from Schofield [15].

the eigenstates of the interacting system can be mapped one-to-one or adiabatically continued onto the eigenstates of the non-interacting system [12, 13, 14]. This is illustrated in a cartoon fashion in Figure 1.6, taken from Schofield [15]. In this case, the "non-interacting" system is the infinite square well, and the "interacting" system is the parabolic potential well. Although the eigenstates of the parabolic potential are clearly distinct from and inhabit a different Hilbert space than those of the square well, if we use the number of nodes in the wavefunction as a method of labeling the eigenstates, we find that the eigenstates of the parabolic well can be mapped monotonically and one-to-one onto the eigenstates of the "non-interacting" square well. A number of consequences extend naturally from Landau Fermi liquid theory, such as Luttinger's sum rule [16] and having zero scattering rate or infinitely long lived quasiparticles at $\mathrm{E}_{\mathrm{F}}$ (neglecting impurity scattering).

While the combination of one-electron band theory and Landau Fermi liquid theory provides an excellent starting point for understanding the solid-state, there are many instances where band theory breaks down. Perhaps the first realization of this came in 1937, when deBoer and Verwey reported the case of transition metal 
oxides which had partially filled $d$-bands and were expected to be metals but were often insulators or poor conductors [17]. It was then suggested by Peierls and Mott that the electrostatic interaction between the conduction electrons led to insulating behavior [18], similar to the ideas proposed by Wigner in 1934 [19] on Wigner crystallization. In the following years, the concept of the Mott insulator was further developed $[20,21,22]$. The importance of the electron-electron interactions should not be surprising, since the electron-electron interaction term is $\sum_{j \ll k}^{N_{e}} \frac{e^{2}}{\left|\vec{r}_{j}-\vec{r}_{k}\right|}$, which can be comparable to the kinetic energy term, $\sum_{j}^{N_{e}} \frac{\hbar^{2}}{2 m} \nabla_{j}^{2}$, and therefore can often make the non-interacting Fermi gas a poor choice for a starting ground state. This is obviously the case for the parent high- $\mathrm{T}_{\mathrm{c}}$ cuprates, since the Mott insulating state cannot be arrived at perturbatively from a starting ground state of the non-interacting Fermi gas.

Although the effects of electron correlations in solids was certainly an active field in the years following the initial work of Mott, this topic vaulted to the forefront of the solid-state physics field with the discovery of spectacular changes in the physical properties of certain transition metal oxides, such as the high- $T_{c}$ cuprates and the colossal magnetoresistive manganites. One common thread in many of these strongly correlated transition metal oxides is that many of the half-filled compounds which are predicted by band theory to be metals are, in fact, insulators.

While the parent compounds of the cuprates are often loosely referred to as "Mott insulators", they should in fact be considered as "charge-transfer insulators" as denoted from the famous Zaanen-Sawatzky-Allen (ZSA) [23] classification of 3d transition metal compounds. The key distinction is that Mott insulators refer to an on site Coulomb interaction which gives rise to the insulating behavior; for the insulating cuprates, this would mean that both the first electron removal and addition states have $3 d$ character, such that the insulating gap, $U$, is of the $d-d$ type $\left(d_{i}^{n} d_{j}^{n} \rightarrow d_{i}^{n+1} d_{j}^{n-1}\right)$. However, for the case of the cuprates, it is known that the first electron removal state is on the O $2 p$ ligand orbital. Therefore, the insulating gap is associated with the charge transfer energy, $\Delta$, between the ligand orbital and the transition metal ion $\left(d_{i}^{n} \rightarrow d_{i}^{n+1} \underline{L}\right)$.

The fact that the charge transfer between the $\mathrm{O} 2 p$ and $\mathrm{Cu} 3 d$ orbitals is critical to 


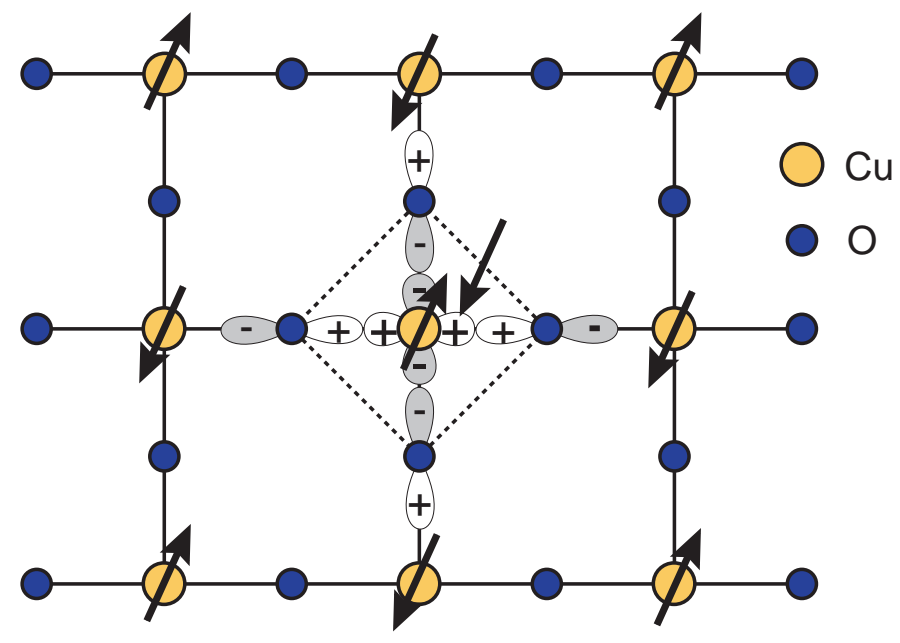

Figure 1.7: Schematic showing the proposed Zhang-Rice singlet wavefunction, with a single hole injected into the antiferromagnetic $\mathrm{CuO}_{2}$ plane.

describing the properties of the cuprates suggests that a three-band model, where the $\mathrm{Cu} 3 d_{x^{2}-y^{2}}$, O $2 p_{x}$, and $\mathrm{O} 2 p_{y}$ orbitals are explicitly considered, is the best description of the low-energy electronic structure of the cuprates. Early in the history of the field, Zhang and Rice suggested that the lowest-lying electron removal state was that the hole delocalized on the four oxygen $2 p$ orbitals surrounding the $\mathrm{Cu}^{2+}$ ion, and hybridized with the $\mathrm{Cu} 3 d_{x^{2}-y^{2}}$ orbital, forming a singlet state [24].

This work by Zhang and Rice allowed the further simplification of the three-band model into a single-band of Zhang-Rice singlets, suggesting that one might neglect the internal orbital structure of this lowest-lying state. By doing so, one can effectively recover a simplified single-band Hubbard model where the charge-transfer insulator is treated as a Mott insulator. In this case, the effective "lower Hubbard band" is the largely $\mathrm{O} 2 p$ derived Zhang-Rice singlet-like band, the "upper Hubbard band" is a primarily $\mathrm{Cu} 3 d^{10}$ derived band, and the "Mott gap" is the $\mathrm{Cu}-\mathrm{O}$ charge-transfer energy, $\Delta \sim 2 \mathrm{eV}$. Throughout this thesis, we will use this terminology interchangably, and most of the existing literature on the cuprates typically use the Mott-Hubbard terminology. There is, in fact, little experimental evidence that the Zhang-Rice singlet state is actually the lowest-lying electron removal state. Recent spin-resolved 
photoemission experiments have confirmed that the lowest-lying electronic states indeed have a predominantly singlet character, consistent with the $3 d^{9} \underline{L}$ Zhang-Rice singlet character [25]. As we will discuss in Appendix B, we believe that while the single band model may be sufficient for discussing the general behavior of the lowlying excitations, the hybridization of the $\mathrm{Cu} 3 d$ and $\mathrm{O} 2 p$ orbitals (which would be included in the three-band model) is necessary for a truly accurate description of the single-particle excitations, and particularly matrix element effects.

The reduction of the three-band to a single band model allows one to write down the Hamiltonian for a single-band Hubbard model as follows:

$$
\mathcal{H}=-t \sum_{\langle i j\rangle, \sigma}\left(c_{i \sigma}^{\dagger} c_{j \sigma}+\text { h.c. }\right)+U \sum_{i} n_{i \uparrow} n_{i \downarrow}
$$

where $t$ is the kinetic energy term of moving an electron from site $i$ to one of its four nearest neighbors, $j$, and $U$ is the on-site electron Coulomb repulsion. For the cuprates, we will effectively replace the onsite $U$ term with the charge-transfer energy, $\Delta$. However, even this seemingly simple Hamiltonian is impossible to solve exactly, and even further restraints must be placed on this model. In the limit where $U \gg t$, the double occupancy of sites becomes prohibitively costly. In the actual materials, $U \rightarrow \Delta \sim 2 \mathrm{eV}$, and $t \sim 0.35 \mathrm{eV}$, so this may be a reasonable approximation. Then, in this case, we can reduce the Hubbard model to a so-called $t-J$ model, where

$$
\mathcal{H}=-t \sum_{\langle i j\rangle, \sigma}\left(c_{i \sigma}^{\dagger} c_{j \sigma}+\text { h.c. }\right)+J \sum_{\langle i j\rangle, \sigma}\left(\mathbf{S}_{i} \cdot \mathbf{S}_{j}-\frac{n_{i} n_{j}}{4}\right)
$$

where $J$ is the antiferromagnetic exchange interaction and $\mathbf{S}_{i}$ is the on-site spin operator. One can obtain the exchange interaction by considering the lowering of kinetic energy through a virtual hop of an electron to a neighboring site of opposite spin via first-order perturbation theory, where $J=\sum_{j} \frac{\left|\left\langle j\left|t_{i j} c_{j}^{\dagger} c_{i}\right| i\right\rangle\right|^{2}}{U}$.

The key point of the $t-J$ model is that it simplifies the problem through a Gutzwiller projection - that is, by taking the $U \rightarrow \infty$ limit, one removes all doubly occupied states from the Hilbert space. Even under this approximation, exact 
solutions of the $t-J$ model are still impossible. Nevertheless, respectable numerical calculations of the $t-J$ model can be performed on finite clusters (typically on the order of $4 \times 4$ ) to obtain insight into the properties of these strongly correlated systems.

At a certain level, the study of these $t-J$ or Hubbard models is largely academic, and one can simply ask the question of how close the numerical results come to approximating the actual solution of the Hamiltonian. However, a more fundamental question is how close these model Hamiltonians come to describing the real materials in question. For instance, how important is the effect of double occupancy and the fact that $U$ is not infinite? What are the effects of higher order hopping terms $\left(t^{\prime}, t^{\prime \prime}\right)$ or longer-ranged Coulomb interactions? Is it appropriate to neglect the effects of electron-phonon interactions? In Chapter 5 will deal with some of these questions, particularly the effects of electron-lattice interactions in the real materials.

\subsubsection{Properties of the High- $\mathrm{T}_{\mathrm{c}}$ Superconductors}

One of the reasons that many physicists assumed, justifiably, that the high- $\mathrm{T}_{\mathrm{c}}$ superconductors were fundamentally different from the conventional BCS variety was not only their extremely high $\mathrm{T}_{\mathrm{c}} \mathrm{s}$, but also their anomalous physical properties. Shown in Figure 1.8 is the canonical phase diagram of the high- $\mathrm{T}_{\mathrm{c}}$ cuprates, spanning both electron and hole doping. Aside from the unusually high- $\mathrm{T}_{\mathrm{c}} \mathrm{s}$, the most dramatic aspect of the phase diagram is that the parent compounds are antiferromagnetic Mott insulators, clearly signaling the breakdown of Fermi liquid theory upon approaching the underdoped and undoped side of the phase diagram. In fact, the normal state properties of the underdoped cuprates exhibit various non-Fermi liquid or "bad metal" properties, as will be detailed later. In general, the hole doped cuprates heavily outnumber the electron doped cuprates. In the electron doped cuprates, superconductivity is heavily suppressed and exists only in a narrow doping range with fairly low $\mathrm{T}_{\mathrm{c}} \mathrm{s}$. In this thesis, we will focus only on the doping evolution of the hole doped systems, and in particular the cuprate $\mathrm{Ca}_{2-x} \mathrm{Na}_{x} \mathrm{CuO}_{2} \mathrm{Cl}_{2}$, away from the Mott insulator, $\mathrm{Ca}_{2} \mathrm{CuO}_{2} \mathrm{Cl}_{2}$; the doping evolution of the electron doped cuprate $\mathrm{Nd}_{2-x} \mathrm{Ce}_{x} \mathrm{CuO}_{4}$ 


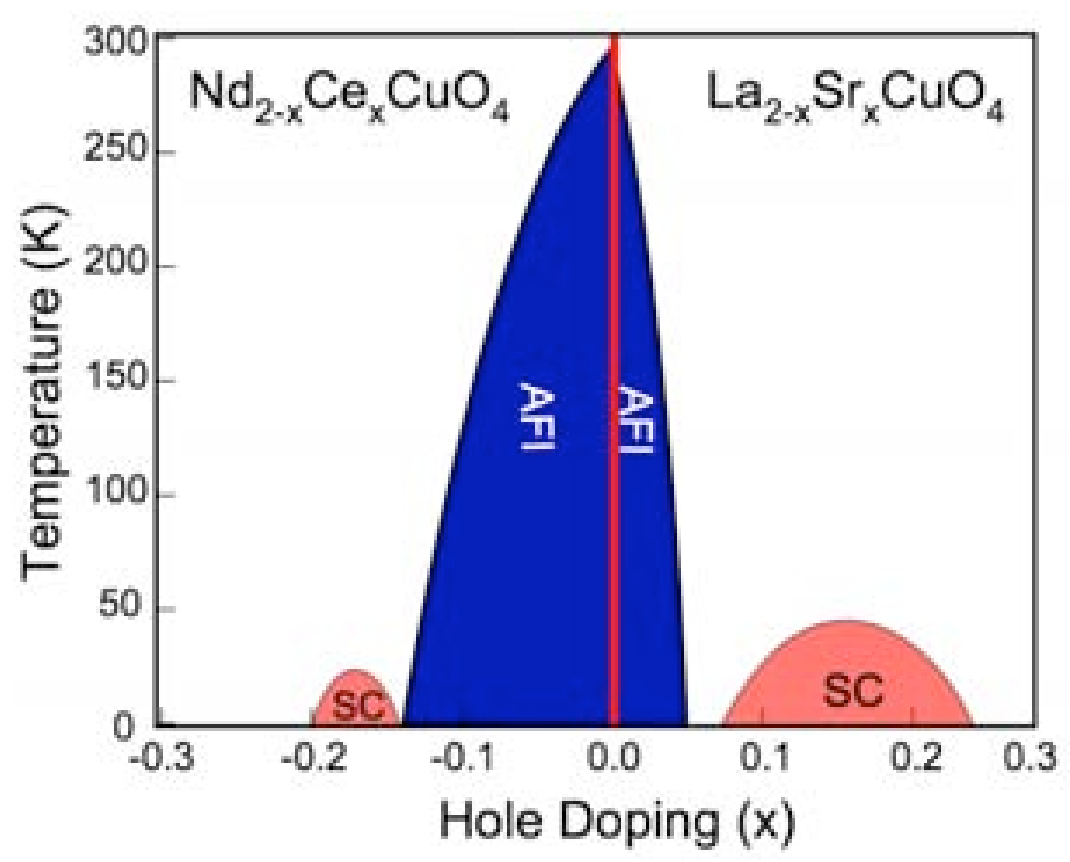

Figure 1.8: Phase diagram of the hole-doped $\left(\mathrm{La}_{2-x} \mathrm{Sr}_{x} \mathrm{CuO}_{4}\right)$ and electron-doped $\left(\mathrm{Nd}_{2-x} \mathrm{Ce}_{x} \mathrm{CuO}_{4}\right)$ cuprates.

is detailed in $[26,27]$.

Long-range antiferromagnetic Néel order persists in a narrow range around halffilling. $\mathrm{T}_{N}$ for typical cuprates is typically around $\sim 300 \mathrm{~K}$ at half-filling, and Néel order vanishes usually around $x>0.03$. We note that because these compounds are highly two-dimensional, $\mathrm{T}_{N}$ reflects the three-dimensional ordering and interlayer coupling strength, while the in-plane, nearest neighbor antiferromagnetic exchange energy $J \sim 150 \mathrm{meV} \sim 1500 \mathrm{~K}$. However, very strong short-range antiferromagnetic correlations persist up to rather high doping levels, even after long range order is destroyed, and a detailed discussion of the magnetic properties of the cuprates can be found in [28]. In addition, some doped cuprates, particularly $\mathrm{La}_{2-x} \mathrm{Sr}_{x} \mathrm{CuO}_{4}$ and its variants ( $\mathrm{Nd}, \mathrm{Ba}$, and Eu-substituted samples) exhibit incommensurate magnetic order which has been attributed to the formation of the doped holes into static onedimensional spin and charge "stripes" [29], potentially explaining why antiferromagnetism remains so robust while at the same time allowing the holes to delocalize and gain kinetic energy [30, 31, 32]. The basic idea of charge ordering (not necessarily as 
one-dimensional stripes) remains a very important and hotly debated topic in the field of high- $\mathrm{T}_{\mathrm{c}}$ superconductivity. Charge ordering can be seen as one particular example of a more global concept of competing orders in the cuprate superconductors. The intervening region between the Mott insulator and the top of the superconducting dome is often to referred to as the "pseudogap" region where the cuprates exhibit a wide array of anomalous normal state properties in the magnetic and charge sectors which are consistent with the opening of some kind of gap, although without the presence of any obvious long-range order. The term "pseudogap" borrows its name from the field of charge-density-wave systems where gapped behavior would manifest itself well before the phase transition itself, due to the formation of incipient short-range CDW order. This is mainly because of the large degree of anisotropy in the 1D Peierls systems where CDW ordering is driven by the one-dimensionality of the system, while the phase transition to the long-range ordered CDW state requires three-dimensional order, caused by interchain coupling. The pseudogap in the cuprates is often attributed to the formation of preformed Cooper pairs above the ordering temperature, due to an extremely strong pairing interaction. In this case, the ordering transition is related to the onset of phase coherence of the preformed pairs. Another viewpoint that we have already mentioned is that the pseudogap is representative of another ordered phase, such as charge ordering or orbital currents [33], which competes with superconductivity and is therefore responsible for the anomalous normal state properties and also for driving down $\mathrm{T}_{\mathrm{c}}$ in the underdoped regime. A comprehensive overview of the pseudogap in the cuprates can be found in the review by Timusk and Statt [34].

In the following, we briefly summarize some of the normal state charge properties of the underdoped cuprates. Perhaps one of the most vaunted aspects of the underdoped cuprates was the approximately T-linear resistivity, which was interpreted to be a clear sign of non-Fermi liquid behavior, which should have a scattering rate $\propto \mathrm{T}^{2}$. Moreover, the evidence of T-linear behavior was often taken to imply that electronphonon interactions (which typically give a $\mathrm{T}^{5}$ term) were also unimportant, although such resistivity data are often difficult to interpret literally. Recent work by Ando et al. on lightly doped $\mathrm{La}_{2-x} \mathrm{Sr}_{x} \mathrm{CuO}_{4}$ show a surprisingly high mobility per carrier 


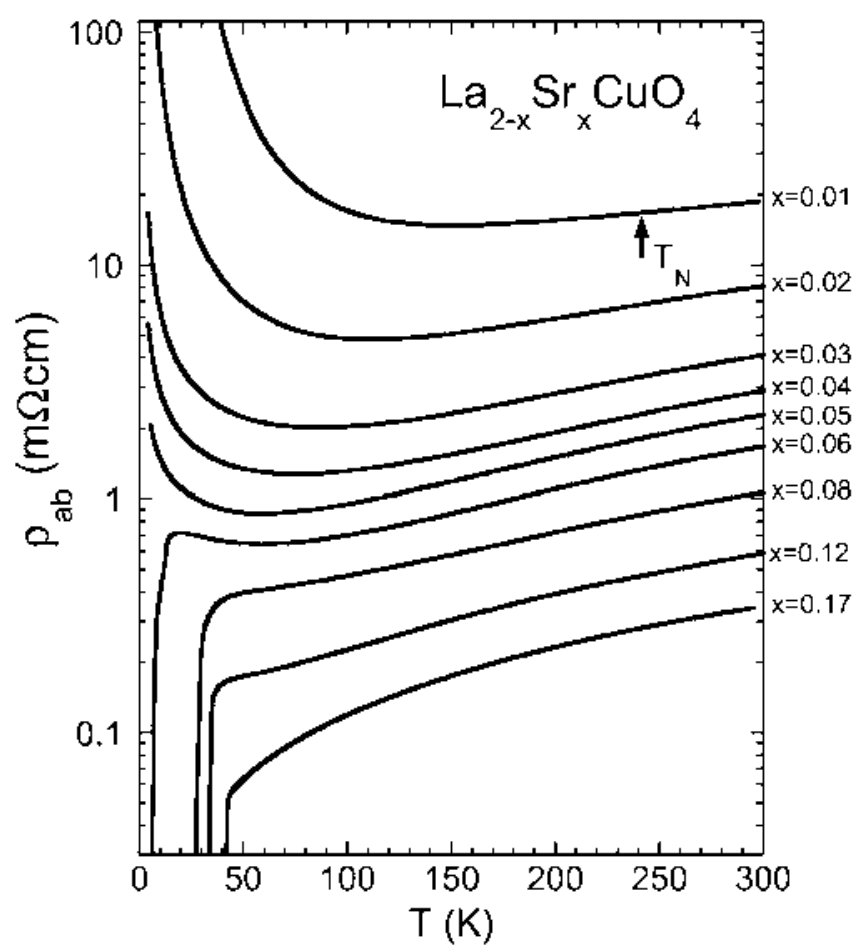

Figure 1.9: Resistivity of $\mathrm{La}_{2-x} \mathrm{Sr}_{x} \mathrm{CuO}_{4}$ from $x=0.01$ to 0.17, from Ando et al. [36].

and high temperature metallic behavior for $x=0.01$ even below $\mathrm{T}_{N}$ where the material should be an antiferromagnetic insulator! At low temperatures, the most lightly doped compositions $(x<0.05)$ are insulating and the heavily underdoped compounds experience an insulating upturn before going superconducting, and a crossover to a $\mathrm{T}=0$, field-induced metallic state is found near optimal doping inside the SC dome [35]. The most recent data on high quality single crystals of $\mathrm{La}_{2-x} \mathrm{Sr}_{x} \mathrm{CuO}_{4}$ are shown in Figure 1.9.

Optical conductivity measurements of $\mathrm{La}_{2-x} \mathrm{Sr}_{x} \mathrm{CuO}_{4}$ have shown that the undoped insulator has a charge-transfer gap of $\sim 2 \mathrm{eV}$, although this values varies considerably from compound to compound. As $\mathrm{La}_{2-x} \mathrm{Sr}_{x} \mathrm{CuO}_{4}$ is doped, a small Drude peak appears at low energy and spectral weight fills into the mid-infrared region between the Drude peak and the charge transfer gap. The optical conductivity data are shown in Figure 1.10, along with a plot of the extracted Drude intensity, both from [37]. This obviously implies that the charge transfer gap does not immediately 

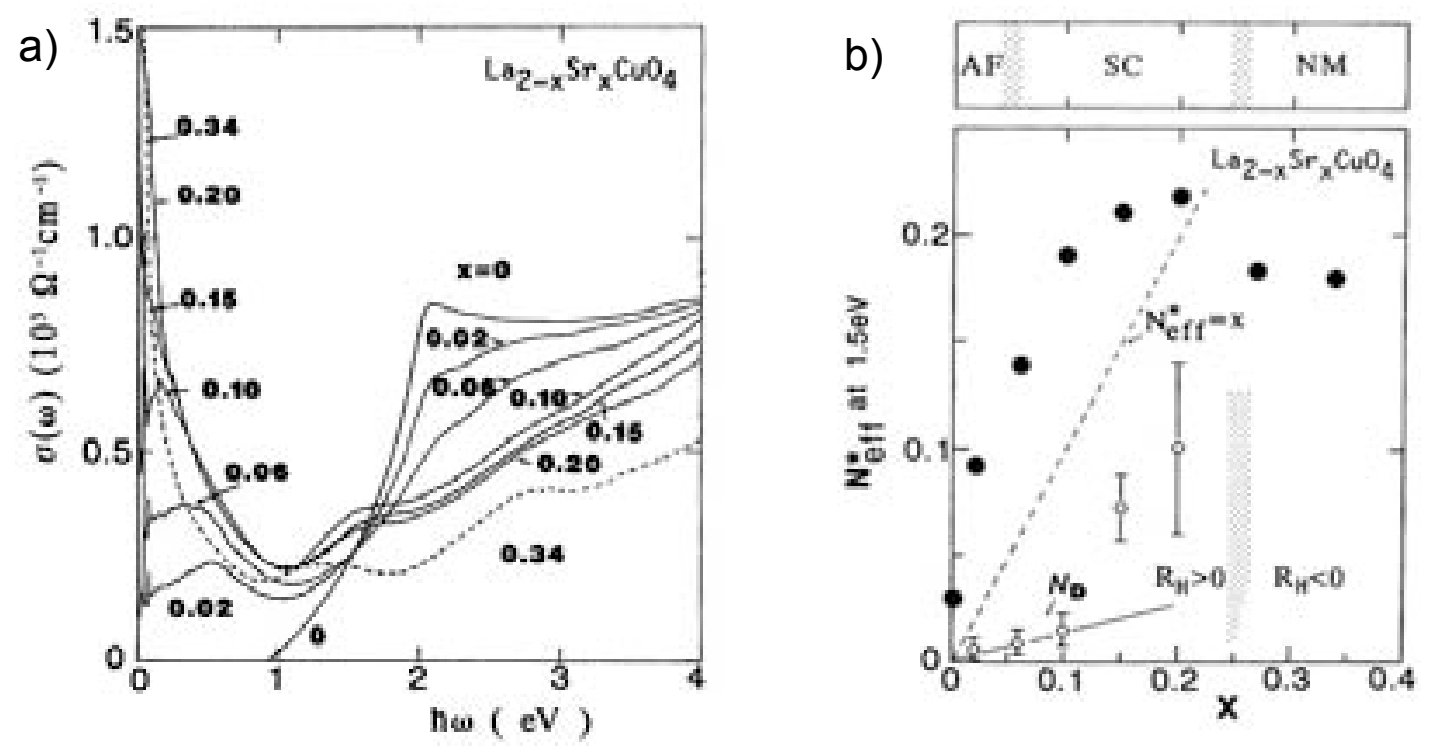

Figure 1.10: (a) Optical conductivity $\sigma(\omega)$ of $\mathrm{La}_{2-x} \mathrm{Sr}_{x} \mathrm{CuO}_{4}$ as obtained from Kramers-Kronig transformation of the $\mathrm{E} \perp \mathrm{c}$ reflectivity. (b) Effective electron number $\mathrm{N}_{\text {eff }}^{*}$ at $1.5 \mathrm{eV}$ and the Drude weight, $\mathrm{N}_{D}$ as a function of doping. At low doping levels, $\mathrm{N}_{D}$ is found to increase proportionally to $x$. From Uchida et al. [37].

collapse upon doping, and the material remains strongly correlated with a large onsite Coulomb repulsion, even if there are metallic carriers at $\mathrm{E}_{\mathrm{F}}$.

The superconducting properties of the hole doped cuprates are also highly anomalous. Perhaps the most distinctive feature of the high- $\mathrm{T}_{\mathrm{c}}$ cuprates, aside from the high transition temperatures, is the fact that the symmetry of the superconducting gap has a $d_{x^{2}-y^{2}}$ symmetry, as opposed to the isotropic $s$-wave gap found in virtually all conventional superconductors. Out of the vast number of known superconductors, only a small handful have been confirmed (or have sufficient evidence to be considered as) non- $s$ wave superconductors, including the cuprates, $\mathrm{Sr}_{2} \mathrm{RuO}_{4}$, and some heavy fermion and organic superconductors. However, virtually all other known non- $s$ wave superconductors have low $\mathrm{T}_{\mathrm{c}} \mathrm{s}\left(\mathrm{T}_{\mathrm{c}}<10 \mathrm{~K}\right)$, so the high- $\mathrm{T}_{\mathrm{c}} \mathrm{s}$ are truly unconventional in this sense. In fact, many conventional superconductors can have gapless excitations, or at least variations in the gap size at different points on the Fermi surface. However, the important question is whether the phase of the superconducting order parameter changes sign at any point in $\mathbf{k}$ space. In that sense, the $d_{x^{2}-y^{2}}$ superconducting gap 


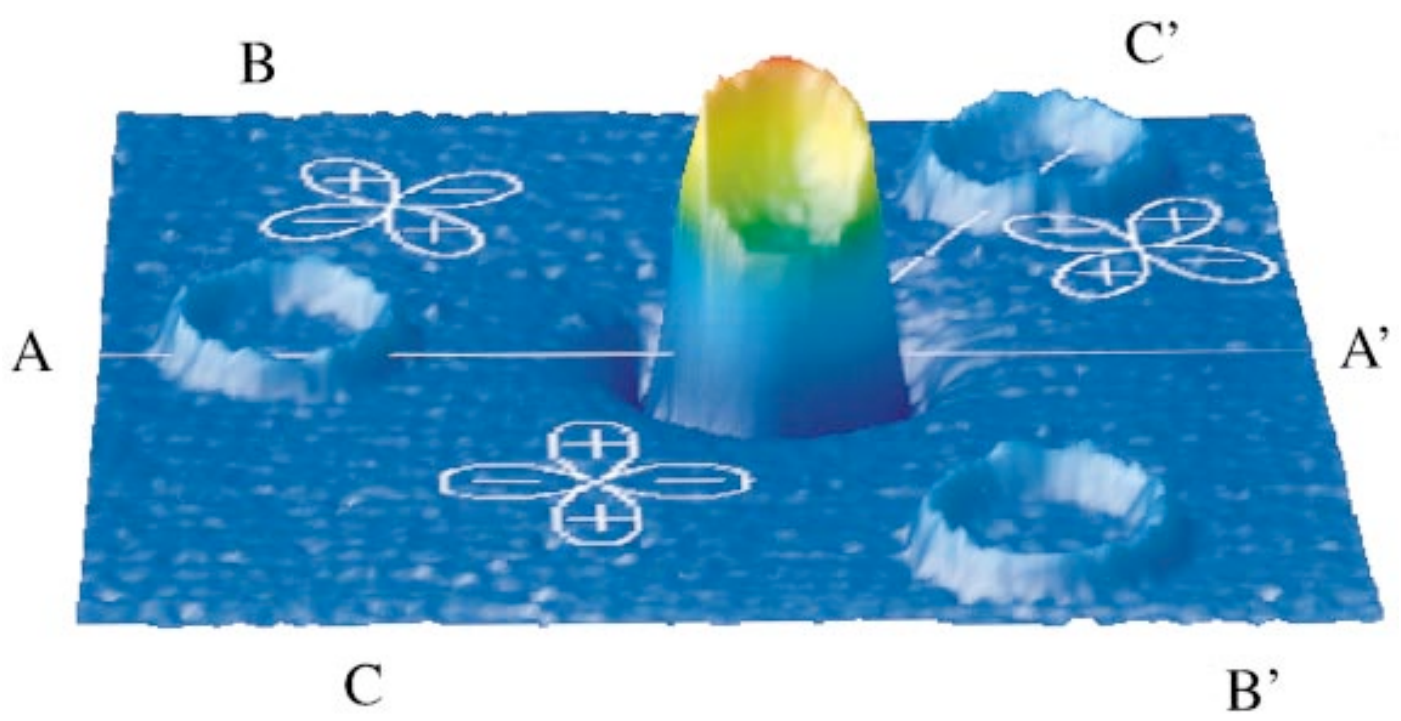

Figure 1.11: A half-flux quantum spontaneously generated at a tricrystal junction, demonstrating the $d_{x^{2}-y^{2}}$ phase of the order parameter. From Tsuei and Kirtley [38].

changes sign four times (every $\pi / 2$ radians) as one goes around the Fermi surface. The superconducting gap maximum is along the $(\pi, 0)$, or $\mathrm{Cu}-\mathrm{O}$ bond direction, while there is a node in the superconducting gap along the $(\pi, \pi)$ direction (diagonal to the $\mathrm{Cu}-\mathrm{O}$ bond). This means that even deep in the superconducting state (in the $\mathrm{T}=$ 0 limit), there are gapless quasiparticles that can be excited, coexisting with the superfluid condensate. The $d$-wave nature of the cuprates was confirmed dramatically by phase-sensitive experiments of Tsuei and Kirtley, shown in Figure 1.11.

Another aspect of the unusual superconducting state is the very low superfluid density (i.e. the total number of electrons participating in the condensate) in the underdoped regime. $\mu \mathrm{SR}$ experiments from Uemura et al. [39] resulted in the so-called "Uemura plot" which showed that the superfluid density scaled with $T_{c}$, suggesting that a large number of electrons are still not able to condense into the superfluid. This might imply that in the underdoped regime, the effects of strong correlations prevent a large $(1-x)$ number of electrons of participating in the low-energy physics and thus cannot form part of the condensate. 


\subsubsection{Key Experimental Results from ARPES}

Photoemission spectroscopy has played a critical role in shaping our understanding of the cuprate superconductors [40], but the two fields have emerged in parallel, and it is difficult to say whether photoemission has had more impact on the cuprates, or vice versa. One reason for the prominence of photoemission in the cuprates was the lack of any other Fermi surface probes, such as de Haas-van Alphen or Shubnikov-de Haas oscillations. This is because the cuprates are doped oxides - notoriously dirty materials and therefore the electronic scattering rate is too high to support such quantum oscillation experiments. However, very recently angular magnetoresistance oscillation experiments have been performed in heavily overdoped $\mathrm{Tl}_{2} \mathrm{Ba}_{2} \mathrm{CuO}_{6+\delta}$ which have found a coherent three-dimensional Fermi surface [41] which corresponds rather closely to the Fermi surface deduced from ARPES [42]. In addition, because of the nature of the strong interactions electron-electron (and electron-boson) interactions in the cuprates, understanding the excitation spectrum at higher energies, not just at zero energy $\left(E_{F}\right)$, is desirable in order to address, for instance, the frequency-dependent scattering rate or transfers of spectral weight with doping, something impossible from transport or quantum oscillation experiments.

Even with the previous generation of electron analyzers, ARPES was able to contribute significantly to our understanding of the cuprates. In the early days of the cuprates, it was believed that the materials were so exotic and non-Fermi liquid-like, that they possessed no well-defined single-electron excitations. The observation of dispersive peaks by Olson [43] and Takahashi [44] was therefore an extremely important discovery. Perhaps the greatest single contribution to the field of high- $\mathrm{T}_{\mathrm{c}}$ superconductivity from ARPES was the detection of the anisotropic $d_{x^{2}-y^{2}}$ superconducting gap in $\mathrm{Bi}_{2} \mathrm{Sr}_{2} \mathrm{CaCu}_{2} \mathrm{O}_{8+\delta}$ by Wells [45] and Shen [46] in 1993. At that time, there was little consensus as to the symmetry of the superconducting gap, and most believed that the cuprates were $s$-wave superconductors. Together with the microwave conductivity measurements of Hardy [47], ARPES was instrumental in turning the tide towards $d$-wave pairing in the cuprates. The detection of the $d$-wave gap by ARPES was rather fortunate, since the experimental resolution at the time was $\sim 20-30 \mathrm{meV}$ 

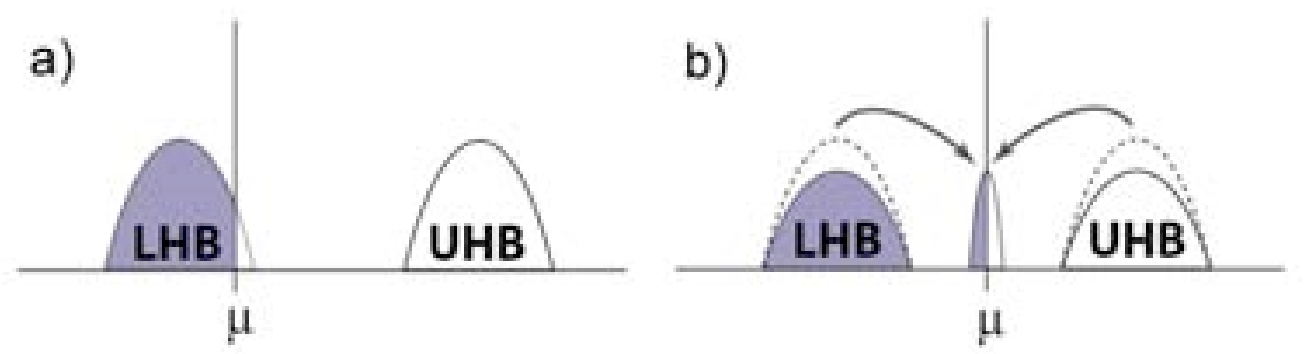

Figure 1.12: Schematic of the doping evolution for (a) a shifting chemical potential, as in a heavily doped band insulator, and (b) a pinned chemical potential forming mid-gap states, as in a lightly doped semiconductor.

and $\mathrm{Bi}_{2} \mathrm{Sr}_{2} \mathrm{CaCu}_{2} \mathrm{O}_{8+\delta}$ has a rather high transition temperature and a resulting superconducting gap of $\sim 40 \mathrm{meV}$. If such experiments were performed on a material such as $\mathrm{La}_{2-x} \mathrm{Sr}_{x} \mathrm{CuO}_{4}$, the $d$-wave gap would likely have eluded detection. After the detection of the $d$-wave $\mathrm{SC}$ gap, work on underdoped $\mathrm{Bi}_{2} \mathrm{Sr}_{2} \mathrm{CaCu}_{2} \mathrm{O}_{8+\delta}$ revealed that the $d$-wave gap did not close above $\mathrm{T}_{\mathrm{c}}$, as one would expect above the phase transition temperature $[48,49]$ and exhibited pseudogap behavior.

In 1995, the single-electron excitation spectrum of the undoped parent compound $\mathrm{Sr}_{2} \mathrm{CuO}_{2} \mathrm{Cl}_{2}$ was studied by Wells [50], and showed qualitatively good agreement with calculations from the $t-J$ model. Later spin-polarized photoemission work verified that the electronic states closest to $\mathrm{E}_{\mathrm{F}}$ have a predominantly singlet character [25, 51], as first predicted by Zhang and Rice [24]. In addition, a great deal of work was also centered on the study of the doping dependence of the chemical potential, $\mu$, since this is a fundamental thermodynamic quantity that could be directly compared with theoretical predictions. However, different studies of the chemical potential often gave conflicting results, including reports that the chemical potential was either pinned in mid-gap upon doping [52, 53], or that it shifted to the top of the valence band (bottom of the conduction band) upon hole (electron) doping $[54,55,26,56]$, posing a significant problem for the understanding of the Mott insulator-to-superconductor transition in the cuprates. These two scenarios for the evolution of the chemical potential are shown in Figure 1.12. Later, with the advent of sophisticated two-dimensional electron analyzers, dispersion anomalies were 
observed, first in $\mathrm{Bi}_{2} \mathrm{Sr}_{2} \mathrm{CaCu}_{2} \mathrm{O}_{8+\delta}[57,58]$, and later in a wide variety of cuprates [59]. This demonstrated a fairly strong coupling of the electrons to a bosonic field.

In this thesis, we will synthesize many of the topics listed above - the spectral features in the undoped Mott insulator, the doping dependence of the chemical potential, and strong electron-boson coupling - into a coherent framework by which to understand the doping evolution of the electronic structure of the cuprates. In particular, we can describe the spectra of the Mott insulator using a model based on small polaron formation, thereby naturally explaining how the quasiparticle residue, $Z$, evolves from near zero in the parent Mott insulator. 


\section{Chapter 2}

\section{Photoemission Spectroscopy}

Angle-resolved photoemission spectroscopy is a technique which has rather illustrious roots. The explanation of the photoelectric effect by Albert Einstein during his "miracle year" of 1905 has been widely heralded as one of the greatest scientific breakthroughs in human history. While his work on relativity captured more of the spotlight and the public's imagination, it is "especially for his discovery of the law of the photoelectric effect" for which he was awarded the 1921 Nobel Prize. Moreover, the elucidation of the photoelectric effect opened the door to the quantum world, which has formed our basis for understanding all of modern science.

The actual photoelectric effect was discovered by Hertz in 1887 and also found by others (Hallwach, 1888 and von Lenard, 1900). The photoelectric effect was inexplicable under Maxwell's electromagnetic wave formulation of light, where the square of the amplitude or intensity of the light wave (the magnitude of the Poynting vector), and not the frequency, represented the energy of the incident light. However, it was found that the maximum kinetic energy of electrons emitted from a clean metallic cathode when illuminated with ultraviolet light went as

$$
E_{k i n, \max }=h \nu-\phi
$$

where $h$ was Planck's constant and $\nu$ was the frequency of the incident light, and $\phi$ was the work function of the cathode, but nowhere does the intensity of the 
incident light beam factor into this relationship. This led Einstein to postulate that light was quantized, and that each photon carried an energy $h \nu$. Ironically, while Einstein's special and general theories of relativity overturned the Newtonian universe, the explanation of the photoelectric effect revived Newton's concept of the corpuscular nature of light.

\subsection{General Aspects of Photoemission}

It was not until the late 1950's and early 1960's that it was observed that the photoelectric effect could yield interesting information on the nature of the electronic states of the illuminated cathode. Owing to the conservation of energy, which also led to Equation 2.1, it was noticed that the energy distribution of photoemitted electrons could provide information on the density of electronic states in the cathode material. In particular, the 1964 photoemission work of Berglund and Spicer [60] on $\mathrm{Cu}$ and $\mathrm{Ag}$ showed the edges of the $d$ bands at $2 \mathrm{eV}$ and $4 \mathrm{eV}$ below the Fermi energy, in agreement with the predictions of the non-interacting band theory. Later, Kai Siegbahn would share the 1981 Nobel Prize in Physics for his development of electron spectroscopy.

As early as 1964, Kane argued that the momentum-dependent band structure could be mapped from the angle and energy dependence of the photoemission spectra $[61,62]$. However, during the formative years of photoemission, all experiments were exclusively angle-integrated. In the early 1970's, work began in earnest on measuring the angular distribution of the photoelectrons. It was not until 1974 that the first angular dependent band-mapping was performed using photoemission by Smith, Traum and DiSalvo $[63,64,65,66]$. As a prelude to the current state-of-the-art ARPES experiments in this thesis, Smith et al. also used the two-dimensional layered compounds $\mathrm{TaS}_{2}$ and $\mathrm{TaSe}_{2}$ to avoid the complications of the uncertainty in the transverse momentum, $k_{z}$, and were able to show good agreement with band structure calculations $[65,66,67]$.

However, the energy resolution of photoemission experiments in the late 1970's and 1980's were on the order of $100 \mathrm{meV}$. In contrast, the thermodynamic properties 


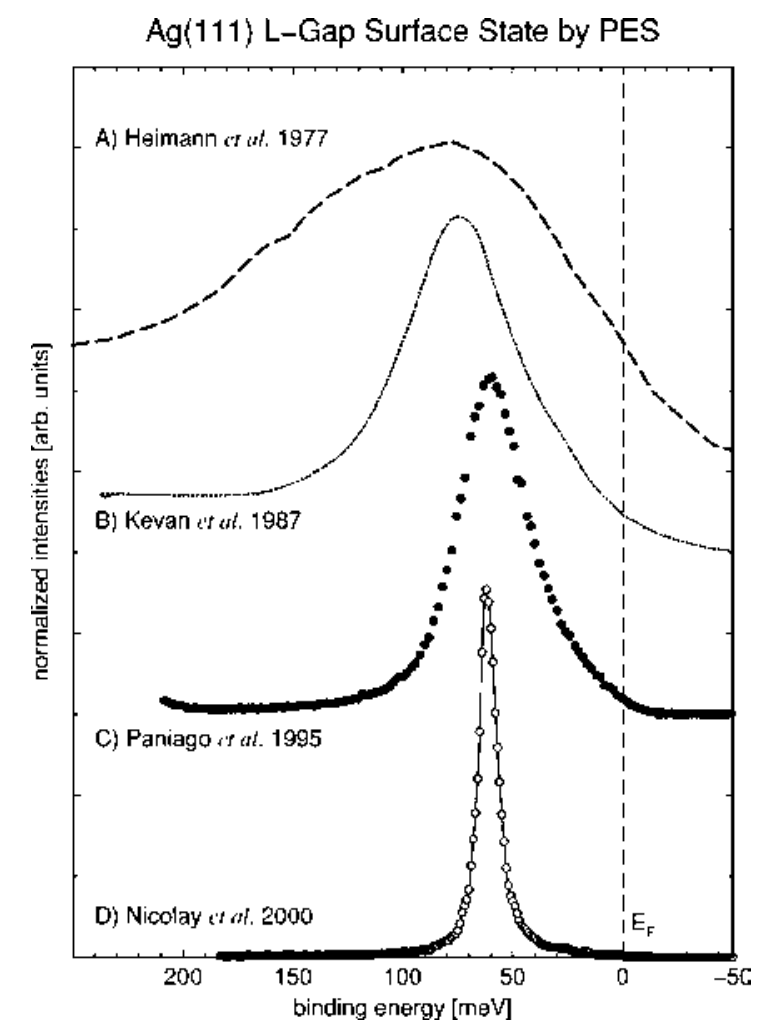

Figure 2.1: Progression of the $L$-gap surface state for $\mathrm{Ag}(111)$ with time, showing the vast improvements in instrumental resolution. For data at the bottom, the peak width is due primarily to intrinsic lifetime broadening, and not instrumental resolution. In fact, the improvements stem not only from instrumental resolution, but also improvements in the surface quality as well as lowered measuring temperatures. From [68].

of solids are determined by the electronic states within a thin strip of energy about $k T$ wide around the Fermi energy. At room temperature, this corresponds to a thermal energy of $30 \mathrm{meV}$, but at low temperatures where phenomena such as superconductivity occur $(\sim 10 \mathrm{~K})$, this corresponds to an energy of roughly $1 \mathrm{meV}$. Therefore, an energy resolution of $\sim 100 \mathrm{meV}$ (corresponding to roughly $1000 \mathrm{~K}$, on the order of the melting temperature of most solids) was clearly insufficient to address anything beyond the gross electronic structure of solids. However, substantial advances in detector technology in the late 1990's and early 2000's resulted in order-of-magnitude improvements in the energy and angular resolutions, along with the data acquisition 
efficiency. The chronology of improvements in the resolution of electron spectroscopy is shown in Figure 2.1. At present, multichannel angular and energy detection in normal ARPES experiments typically occur using $\Delta E \sim 10 \mathrm{meV}, \Delta \theta \sim 0.3^{\circ}$, and with 100 angular channels acquired simultaneously. Analysis of ARPES spectra using the latest detector technology will be discussed later in this chapter. Using the latest generation of analyzers and laser-based light sources, sub-meV energy resolutions have recently been demonstrated $[69,70,71]$.

\subsection{Energetics and Kinematics of ARPES}

In Figure 2.2, we show the energetics of the photoemission process in a simplified density-of-states picture. Within the solid, there is an electronic density of states governed by the intrinsic band structure and interaction effects, where states are filled up to the Fermi energy, $\mathrm{E}_{\mathrm{F}}$. As discussed earlier, there is a finite potential energy barrier between the first occupied electronic state $($ at $T=0)$ at $\mathrm{E}_{\mathrm{F}}$, and the vacuum level (the potential energy zero at $x \rightarrow \infty$ ), which is the work function of the material, $\phi$, and this is what holds the electrons inside the crystal. Therefore, when an electron absorbs a photon (and does not experience any inelastic losses), the binding energy of the electron in the initial state relative to the Fermi energy, $E_{B}$, can be related to the measured kinetic energy, $E_{k i n}$, by $h \nu-\phi-E_{F}$. The work function in question is that of the detector, since all materials have slightly different work functions depending on the characteristics of the surface. If the work function of the sample and detector are different, then a potential will be set up between the sample and detector, $\phi_{\text {sample }}-\phi_{\text {detector }}$.

To relate the relationship of the photoelectron momentum to the momentum of the photohole left in the crystal, we consider an electron in a crystal with a welldefined quasimomentum $\mathbf{k}$, and energy $E_{B}$. If the incident photon has enough energy to promote the electron above $E_{v a c}$, then the electron can be photoemitted into free space. Because the electron has to propagate a macroscopic distance $(\sim 1 \mathrm{~m})$ to the detector, it can essentially be treated as a free plane wave with $E=\hbar \mathbf{k}^{2} / 2 m$. Therefore, by knowing the takeoff angles of the electron relative to the crystal axes 


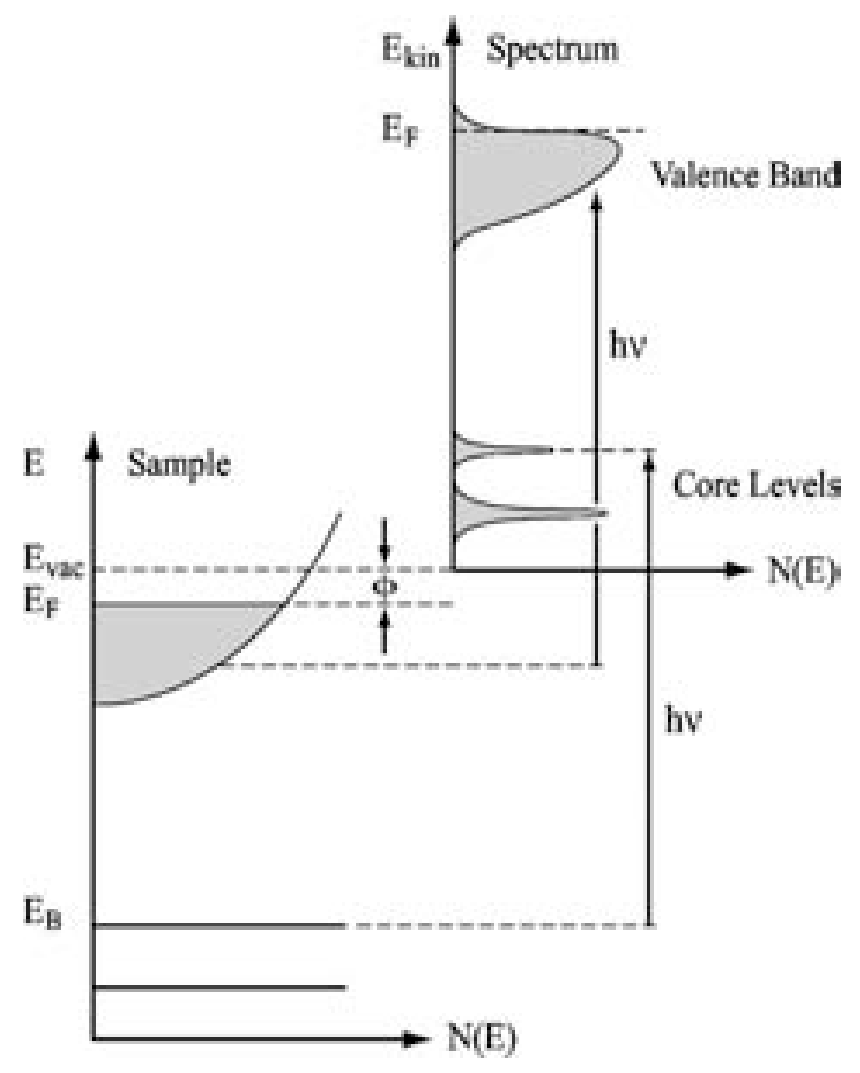

Figure 2.2: On the left, the energy levels in the crystal in the initial state. Photoemission process occurs with the absorption of a photon with energy $h \nu$. On the right, the measured photoemission spectrum, starting from the vacuum level. From [40].

and the detector, we can determine the momentum wavevector of the outcoming photoelectron. From this we can use momentum conservation to relate this to the quasimomentum of the electron in the initial state. We can roughly generalize the real situation to a semi-infinite crystal (infinite in the lateral directions, but with a discontinuous step - the crystal surface - in the $z$ direction). In this case, the momentum in the lateral direction, $\mathbf{k}_{\|}$is conserved due to translational symmetry, but the momentum in the $z$ direction is clearly not conserved, due to the work function step potential at the surface (the lost $\mathbf{k}_{\perp}$ momentum of the photoelectron across the barrier is then taken up by a recoil of the crystal). Therefore, we can obtain an exact measure of the in-plane wavevectors of the electron in the initial state, 

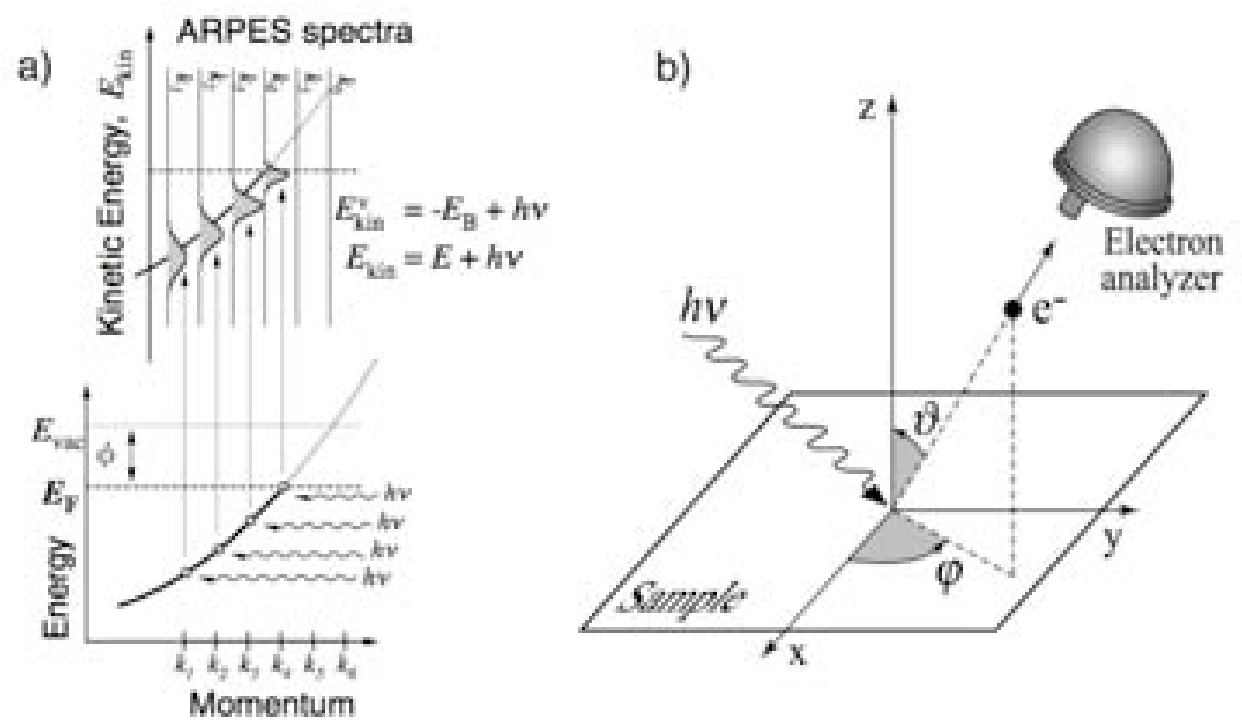

Figure 2.3: (a) Illustration of optical (direct) transitions from electrons in a band in the crystal, to photoelectrons in free space. From [73]. (b) ARPES and measured observables, being photoelectron counts, photoelectron kinetic energy, and polar angles of photoelectron relative to sample crystal axes. From [40].

$\mathbf{k}_{\|}$, but extracting $\mathbf{k}_{\perp}$ is more challenging. To determine $\mathbf{k}_{\perp}$, one should determine the so-called "inner potential" of the crystal, which is essentially determining which photoelectron kinetic energy corresponds to $\mathbf{k}_{\perp}=0$ (the $(0,0,0)$ point) [72]. This is typically rather involved and requires spanning a wide range of incident photon energies, $h \nu$, in our measurements. The measurements related here were performed on highly two-dimensional materials where $\mathbf{k}_{\perp}$ should not be a particularly relevant quantum number, and for the rest of this thesis, we will neglect $\mathbf{k}_{\perp}$.

First, it is most appropriate to view the experimentally measured photocurrent as a photoinduced transition between electron initial and final states. In ultraviolet photoemission spectroscopy (UPS), the incoming photon carries negligible momentum (for $\left.h \nu=10 \mathrm{eV}, \mathbf{q} \sim 5 \times 10^{-3} \AA^{-1}\right)$, so all transitions are essentially direct $(\mathbf{q}=$ $0)$. To reach an available final state, the photoelectron should then be translated by a reciprocal lattice vector, $\mathbf{G}$, so that the wavevector of the outgoing photoelectron $\mathbf{K}=\mathbf{k}+\mathbf{G}$, demonstrating that the photoemission process requires the presence of a lattice potential to conserve momentum. The above analysis allows us to relate 
the photoelectrons measured at some kinetic energy and momentum to electrons with some different binding energy and momentum in the initial state, inside the crystal before the photoemission process. However, it does not tell us the direct relationship between the electronic states of the $N$ and $N-1$ system. For this, one must make further assumptions beyond simple kinematics and conservation relations to obtain meaningful information about the $N-1$ system of the photohole from the experimentally measured photocurrent. Using Fermi's golden rule, the measured photocurrent can be expressed as a transition matrix element

$$
w_{f i}=\frac{2 \pi}{\hbar}\left|\left\langle\Psi_{f}^{N}\left|\mathcal{H}_{\text {rad }}\right| \psi_{i}^{N}\right\rangle\right|^{2} \delta\left(E_{f}^{N}-E_{i}^{N}-h \nu\right)
$$

where $E_{i}^{N}$ is the energy of the $N$ electron system in the initial state, and $E_{f}^{N}=$ $E_{f}^{N-1}+E_{k i n}$ is the energy of the $N$ electron system in the final state, corresponding to the $N-1$ system left behind plus the ejected photoelectron. $\mathcal{H}_{\text {rad }}$ is the interaction of the electron with the radiation field and can be expressed as

$$
\mathcal{H}_{\text {rad }}=-\frac{e}{2 m c}(\mathbf{A} \cdot \mathbf{p}+\mathbf{p} \cdot \mathbf{A}) \approx-\frac{e}{m c} \mathbf{A} \cdot \mathbf{p}
$$

where we consider only the linear term in $\mathbf{A}$ and use the commutation relation $[\mathbf{p}, \mathbf{A}]=-i \hbar \nabla \cdot \mathbf{A}$ and use the dipole approximation to set $\nabla \cdot \mathbf{A}=0$. We note that this neglects effects at the surface-vacuum interface where a large change in the dielectric constant can lead to sizable contributions from $\nabla \cdot \mathbf{A}$. However, the key aspect of our photoemission experiments is to extract information about the excited state $N-1$ electron system from the photoelectrons which propagate into free space.

Rigorously speaking, the best theoretical description of the photoemission process is the so-called "one-step model". In the one-step model, the photoemission process is treated as a single, entirely quantum-mechanical process. The absorption of the photon by an electron, the excitation of the electron to a higher energy state, and the emission of the electron into vacuum are all treated simultaneously, hence the name "one-step model". Furthermore, in the one-step model, one must also take into account the bulk, the surface, changes in the dielectric constant, interference between bulk and surface transitions, scattering of photoelectrons by plasmons, and evanescent 
(damped electrons in the near-field) states, among other things [74, 75, 76]. Therefore, the one-step model, while exact, is not a particularly useful approximation for helping us understand and interpret our experimental spectra, much like Equation 1.1 is not particularly helpful for solving high- $\mathrm{T}_{\mathrm{c}}$. We should note that some model one-step calculations have been performed for the cuprates using sophisticated band structure calculations for the initial and final states [77, 78]. However, these should only be appropriate for materials near the optimally or overdoped side of the phase diagram where $\psi_{i}$ might potentially be reasonably approximated by a Bloch wave obtained from band calculations; clearly, such calculations should fail for the Mott insulating compounds. Secondly, spending a month of supercomputer processing time on an hour's worth of ARPES beamtime is obviously neither a efficient nor practical approach to data analysis. Finally, the motivation for studying many of these problems is to gain information about the nature of the $N-1$ initial state wavefunction. Since the rigorous one-step model requires a priori knowledge of $\psi_{i}$, this makes the one-step model very impractical for the interpretation of ARPES studies of systems where the electronic states are very poorly understood from theoretical grounds. If the initial state wavefunction of the cuprates was already known, then we would have already solved the problem of high-temperature superconductivity.

The phenomenological model which is nearly exclusively used in the photoemission community is the "three-step" model, popularized by Berglund and Spicer in 1964 [60]. The steps in the three-step model are as follows:

1. Optical excitation of the electron inside the solid

2. Transport of the photoelectron to the surface

3. Escape of the photoelectron into the vacuum

The independent steps in the three-step model are shown in Figure 2.4. In this semiclassical picture, only the first step is treated in a quantum mechanically, and the three steps are treated as independent of one another, in obvious contrast to the one-step approach. The beauty of the three-step model is that it actually removes the complicated photoemission process itself from photoemission! The three-step 


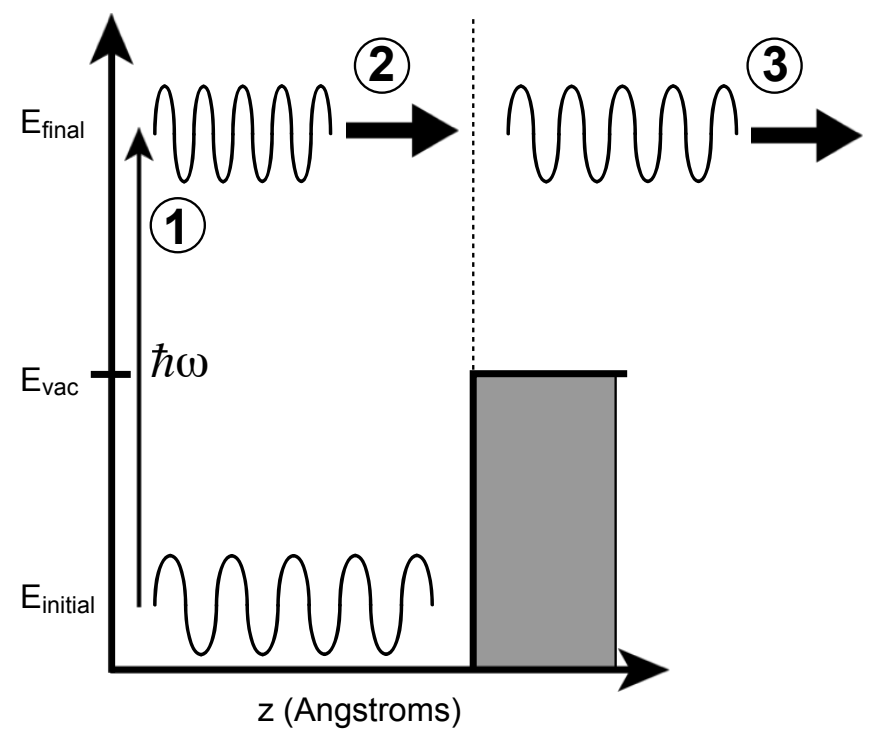

Figure 2.4: Illustration of the three step model, with (1) optical excitation of an electron in the bulk, (2) transport of the photoelectron to the surface, and (3) escape of the photoelectron into vacuum free space.

model effectively reduces the photoemission process to an optical excitation. Steps (2) and (3) in the three-step model are simply attenuation factors and kinematic considerations, respectively, leaving (1) as the only nontrivial process.

Given the dramatic simplifications of the three-step model, it works remarkably well and provides surprisingly accurate results. Certainly, there are many obvious instances where the three-step model breaks down (such as surface-bulk interference on $\operatorname{Ag}(111)$ [79], to name just one example). However, its general reliability even at the level of quantitatively analyzing detailed lineshapes is remarkably good. Here we will discuss a few additional aspects of the photoemission process, before later describing a formal mathematical description of photoemission within the three-step framework. First, photoemission is a highly surface-sensitive process because of the strong Coulomb interactions between the photoelectron and the rest of the electrons left in the solid. In Figure 2.5, we show the well-known "universal curve" plotting the photoelectron mean free path length versus photoelectron kinetic energy. In fact, the "universal curve" is not particularly universal (it is roughly universal, and only 


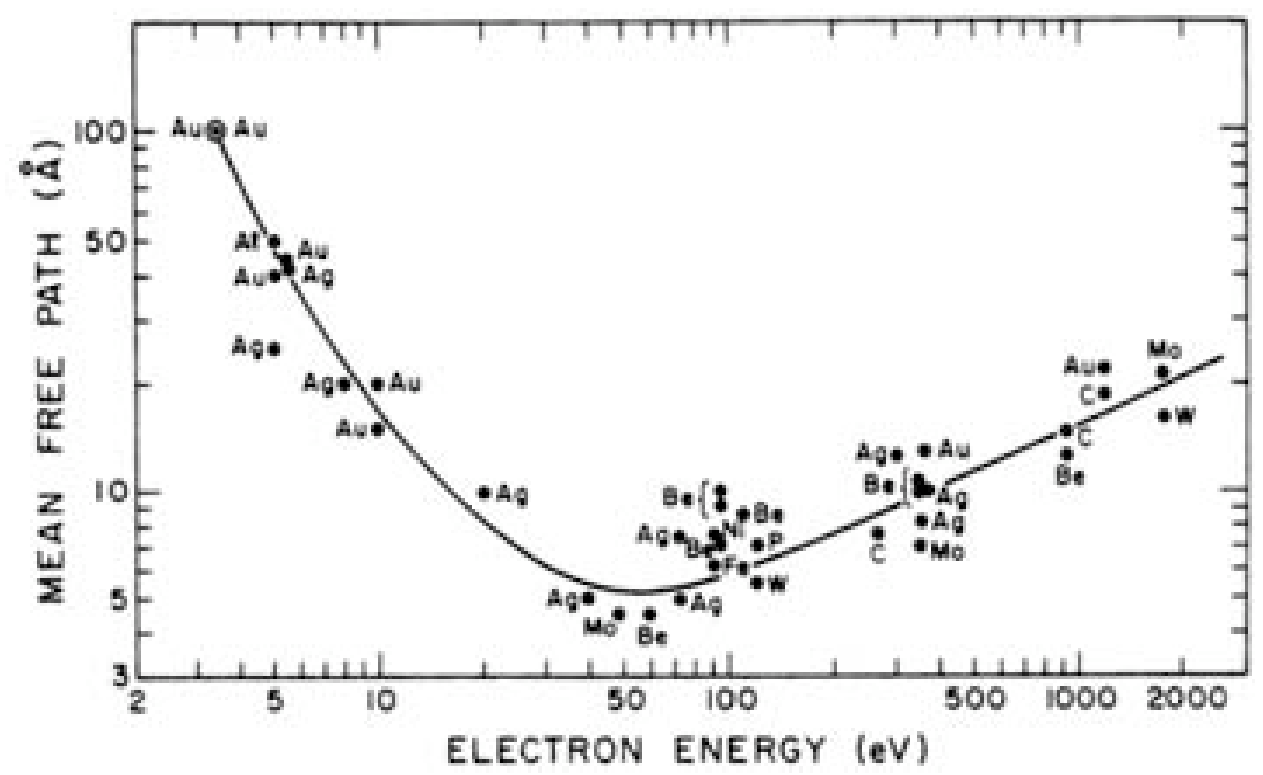

Figure 2.5: The so-called "universal curve" of photoelectron mean free path versus kinetic energy. Taken from Hufner [72] and originally compiled by Seah and Dench [81].

on a logarithmic scale), and was generally compiled using good metals. Because the mean free path depends on the cross-section of the photoelectrons to scatter with plasmons, the mean free path should be even lower for "bad metals" with low plasmon energies such as many strongly correlated transition metal oxides. This has been discussed previously in the cuprates [80], but is an important topic which should be explored further in the field of ARPES on strongly correlated materials, especially at very low kinetic energies. This high degree of surface sensitivity is the reason for extremely stringent ultra-high vacuum conditions necessary for reliable, highresolution ARPES experiments, and we typically perform experiments at pressures of better than $5 \times 10^{-11}$ torr. Samples are usually cleaved in situ at low temperatures and base pressure to ensure an atomically clean surface layer. Even at these pressures, we can occasionally observe surface degradation, although this varies significantly on the particular compound and contaminants in the vacuum chamber. 


\subsection{Spectral Function Interpretation of Photoemission Spectra}

As discussed earlier, we would like to infer information about the details of the electronic states of the $N-1$ electronic system from the experimentally measured photocurrent. The following is a brief summary of how to relate the measured photocurrent to the single particle spectral function, but a more detailed discussion of the photoemission process can be found in the literature [40, 82, 83, 72]. We start with the three-step model and Equations 2.2 and 2.3. We would first like to decompose the $\Psi_{f}^{N}$ final state into a $\Psi_{f}^{N-1}$ state and the outgoing photoelectron. This becomes simple if we assume that the photoemission process is so fast that the photoelectron is ejected instantaneously and the remaining $N-1$ system does not interact with the photoelectron on its way out of the solid, an assumption otherwise known as the sudden approximation [84]. This is a crucial assumption upon which the rest of the following analysis is based. In this case, we can express $\Psi_{f}^{N}=\mathcal{A} \phi_{f}^{\mathbf{k}} \Psi_{f}^{N-1}$ where $\mathcal{A}$ is the antisymmetrization operator for fermions, and $\phi_{f}^{\mathbf{k}}$ is the photoelectron wavefunction with momentum k. For the cuprates, it is generally believed that the sudden approximation should hold for photoelectrons above at least $20 \mathrm{eV}$ [85]. Generally speaking, $\Psi_{f}^{N-1}$ is not an eigenstate of the $N-1$ system, but can be expressed as a superposition of the eigenstates of the $N-1$ system, $\Psi_{m}^{N-1}$, which have energies $E_{m}^{N-1}$

$$
\Psi_{f}^{N-1}=\sum_{m} c_{m} \Psi_{m}^{N-1}
$$

To simplify things, we assume that the initial state can be expressed as a single Slater determinant, at the Hartree-Fock level, so that one can express the initial state as the product of a one-electron orbital $\phi_{i}^{\mathbf{k}}$ and a $N-1$ term, much like we did for the final state wavefunction

$$
\Psi_{i}^{N}=\mathcal{A} \phi_{i}^{\mathbf{k}} \Psi_{i}^{N-1}
$$

In general, any exact many-body wave function can be expressed as a sum of Slater 
determinants such that $\Psi=\sum_{\mathbf{k}} c_{\mathbf{k}} D_{\mathbf{k}}$, as often utilized in "configuration interaction" approaches in quantum chemistry. $D_{\mathbf{k}}$ is a particular Slater determinant, and the $D_{\mathbf{k}}$ S fully span the allowed Hilbert space. For simplicity, we will take the case of just a single Slater determinant for $\Psi_{i}^{N}$, and this can easily be generalized to a case of sum over Slater determinants. $D_{0}$ is the ground state Hartree-Fock reference determinant, and should account for the majority of the exact total energy, while higher order determinants should add smaller and smaller corrections to the ground state energy. So, in general, $\Psi_{i}^{N-1}=c_{\mathbf{k}} \Psi_{i}^{N}$, where $c_{\mathbf{k}}$ is the electron annihilation operator. $\Psi_{i}^{N-1}$ is also not an eigenstate of the $N-1$ system, unless the system is non-interacting. Using the simplification in Equation 2.5, we can then express the matrix element in Equation 2.2 as

$$
\left\langle\Psi_{f}^{N}\left|\mathcal{H}_{\text {rad }}\right| \psi_{i}^{N}\right\rangle=\left\langle\phi_{f}^{\mathbf{k}}\left|\mathcal{H}_{\text {rad }}\right| \phi_{i}^{\mathbf{k}}\right\rangle\left\langle\Psi_{m}^{N-1} \mid \Psi_{i}^{N-1}\right\rangle
$$

since we need to sum over different final states, this allows us to express the measured photocurrent as

$$
I\left(\mathbf{k}, E_{k i n}\right)=\sum_{f, i}\left|M_{f, i}^{\mathbf{k}}\right|^{2} \sum_{m}\left|c_{m, i}\right|^{2} \delta\left(E_{k i n}+E_{m}^{N-1}-E_{i}^{N}-h \nu\right)
$$

where $M_{f, i}^{\mathbf{k}}=\left\langle\phi_{f}^{\mathbf{k}}\left|\mathcal{H}_{\text {rad }}\right| \phi_{i}^{\mathbf{k}}\right\rangle$ and is the one-electron dipole matrix element, and $\left|c_{m, i}\right|^{2}=\left|\left\langle\Psi_{m}^{N-1} \mid \Psi_{i}^{N-1}\right\rangle\right|^{2}$ is the overlap of the removal of an electron from the initial state with the eigenstates of the $N-1$ systems. For a non-interacting system, $c_{\mathbf{k}} \Psi_{i}^{N}$ is one of the eigenstates of the $N-1$ system, $\Psi_{m}^{N-1}$, so the emission is then a $\delta$ function peaked at the energy corresponding to $E_{m}^{N-1}$. If there are a discrete number of eigenstates of the $N-1$ system and the system is interacting, then the intensity will be distributed into a series of $\delta$-functions at energies $E_{m}^{N-1}$, as is the case for molecular photoemission, as illustrated in Figure 4.9. In correlated solids, $c_{\mathbf{k}} \Psi_{i}^{N}$ will overlap with a nearly infinite number of eigenstates, such that there will be many finite $\left|c_{m, i}\right|^{2} \mathrm{~s}$.

It turns out that the mathematical forms derived above appear very similar to the imaginary part of the one-electron Green's function, as we will discuss here. The Green's function approach is discussed in many texts, including Mahan [86]. Green's 
functions are widely used in physics as a method of characterizing the response of some system to an impulse. In this example in condensed matter physics, our example of an impulse is the annihilation or creation of an electron with a given quantum number $\mathbf{k}$ at a particular time $t$. The Green's function then represents the probability amplitude to find that electron at a later time $t^{\prime}$. Fourier transforming $t$ into $w$, the Green's function can be expressed as

$$
G^{ \pm}(\mathbf{k}, \omega)=\sum_{m} \frac{\left|\left\langle\Psi_{m}^{N \pm 1}\left|c_{\mathbf{k}}^{ \pm}\right| \Psi_{i}^{N}\right\rangle\right|^{2}}{\omega-E_{m}^{N \pm 1}+E_{i}^{N} \pm i \eta}
$$

where $c_{\mathbf{k}}^{+}$and $c_{\mathbf{k}}^{-}$represent $c_{\mathbf{k}}^{\dagger}$ and $c_{\mathbf{k}}$, respectively. Taking the imaginary part of this Green's function, the so-called single-particle spectral function, one obtains

$$
\mathcal{A}^{ \pm}(\mathbf{k}, \omega)=\sum_{m}\left|\left\langle\Psi_{m}^{N \pm 1}\left|c_{\mathbf{k}}^{ \pm}\right| \Psi_{i}^{N}\right\rangle\right|^{2} \delta\left(\omega-E_{m}^{N \pm 1}+E_{i}^{N}\right)
$$

where $\mathcal{A}^{-}(\mathbf{k}, \omega)$ and $\mathcal{A}^{+}(\mathbf{k}, \omega)$ correspond to the annihilation and creation of one electron and thus to direct and inverse photoemission, respectively. Because of causality, the real and imaginary parts of the Green's function are related through KramersKronig transformation, and therefore knowledge of $\mathcal{A}(\mathbf{k}, \omega)$ provides full knowledge of $G(\mathbf{k}, \omega)$. In the case of the electron Green's function, it can be shown formally that all many-body interactions can be encapsulated into a complex "self-energy"

$$
\Sigma=\Sigma^{\prime}(\mathbf{k}, \omega)+i \Sigma^{\prime \prime}(\mathbf{k}, \omega)
$$

where the real and imaginary parts are again related through Kramers-Kronig and contain all the information about the renormalization and lifetime of excitations, respectively. The Green's function and spectral function can then be expressed in a form known as Dyson's equation

$$
\begin{gathered}
G(\mathbf{k}, \omega)=\frac{1}{\omega-\varepsilon_{\mathbf{k}}-\Sigma(\mathbf{k}, \omega)} \\
\mathcal{A}(\mathbf{k}, \omega)=-\frac{1}{\pi} \frac{\Sigma^{\prime \prime}(\mathbf{k}, \omega)}{\left[\omega-\varepsilon_{\mathbf{k}}-\Sigma^{\prime}(\mathbf{k}, \omega)\right]^{2}+\left[\Sigma^{\prime \prime}(\mathbf{k}, \omega)\right]^{2}}
\end{gathered}
$$


In general, calculating the form of $G(\mathbf{k}, \omega)$ and $\mathcal{A}(\mathbf{k}, \omega)$ for arbitrary interaction Hamiltonians is extremely difficult, and only very few cases can be solved exactly. Generally speaking, one would typically need to use quantum field theoretic approaches to calculating the electron (or hole) propagator for an interacting manybody system, which is well beyond the scope of this thesis. Instead, we will use very simplified and physically intuitive pictures to describe the effects of many-body interactions on the single-particle spectral function. An illustration of the expectations for $\mathcal{A}(\mathbf{k}, \omega)$ in the case of no interactions and weak interactions is shown in Figure 2.6. In the trivial non-interacting case, $\Sigma(\mathbf{k}, \omega)$ is identically zero, and $\mathcal{A}(\mathbf{k}, \omega)$ is simply a branch of $\delta$-function poles which lie on the non-interacting band dispersion, $\varepsilon_{k}$. When interactions are turned on the $\delta$-functions gain some finite, $\omega$-dependent width and the position of the poles shifts from $\varepsilon_{k}$ to $\varepsilon_{k}+\Sigma^{\prime}(\mathbf{k}, \omega)$. When interactions are turned on, the spectral weight encompassed within the pole is reduced from 1 to a reduced factor $Z_{\mathbf{k}}$, as also shown in Figure 2.6.In the language of the photoemission discussion above, this would correspond to $\left|c_{m, i}\right|$ being finite for many different eigenstates $\Psi_{m}^{N-1}$, and not just a single eigenstate. A particular example of this case is the Fermi liquid where the self-energy can be expressed as

$$
\left.\Sigma_{F L}(\mathbf{k}, \omega)=\beta \omega+i \beta\left[\omega^{2}+\left(\pi k_{B} T\right)^{2}\right)\right]
$$

For the Fermi liquid, away from $\mathrm{E}_{\mathrm{F}}$, the quasiparticles acquire a finite lifetime, $\Sigma^{\prime \prime}$, which grows as $\omega^{2}$. The value of $Z_{\mathbf{k}}$ can be determined from linearly expanding $\Sigma$ around the position of the pole, $E_{\mathbf{k}}^{*}=\varepsilon_{\mathbf{k}}+\Sigma^{\prime}(\mathbf{k}, \omega)$. For a small region in $\omega$ around $E_{\mathbf{k}}^{*}$, we can expand as follows

$$
\omega-\varepsilon_{\mathbf{k}}-\Sigma^{\prime}(\mathbf{k}, \omega) \approx \frac{1}{Z_{\mathbf{k}}}\left(\omega-E_{\mathbf{k}}^{*}\right)
$$

where

$$
Z_{\mathbf{k}}=\left[1-\left.\frac{\partial \Sigma^{\prime}}{\partial \omega}\right|_{\omega=E_{\mathbf{k}}^{*}}\right]^{-1}
$$

In this expansion around the vicinity of the quasiparticle pole, 


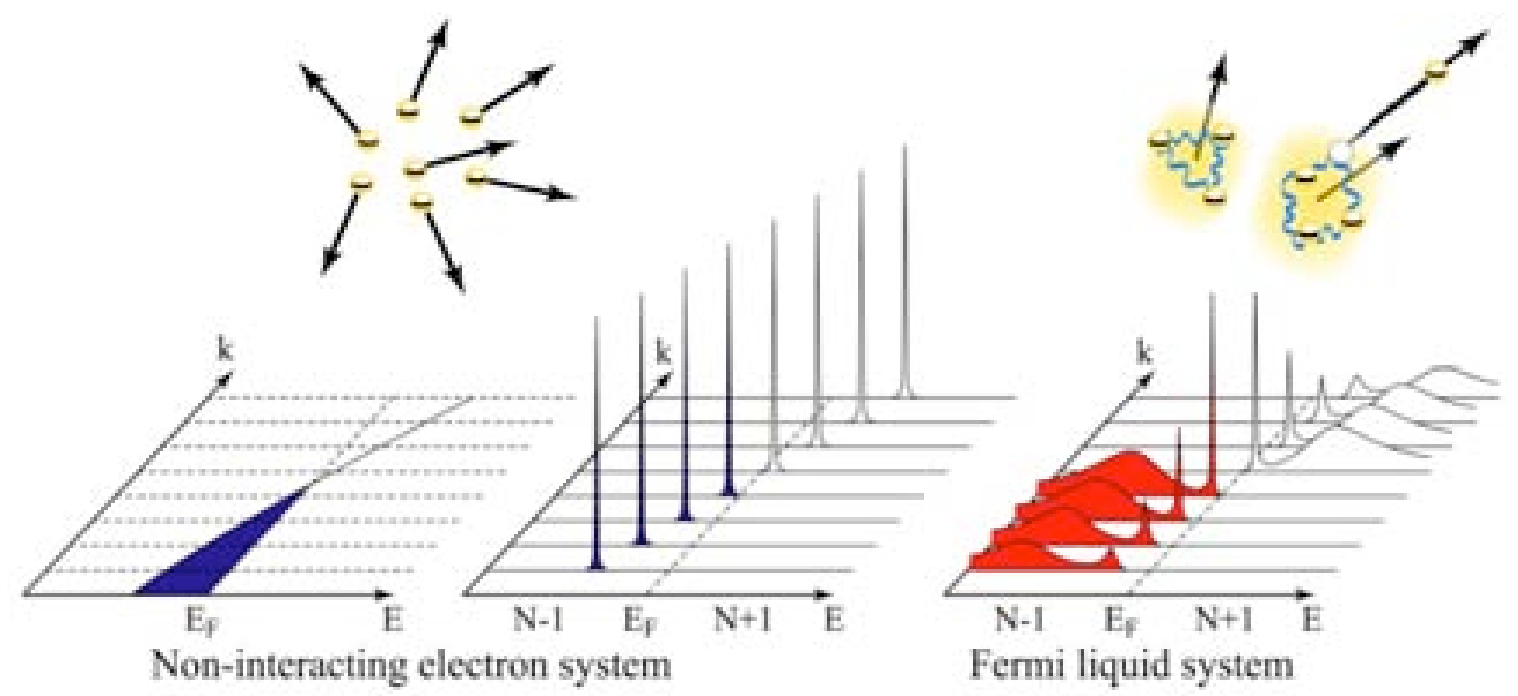

Figure 2.6: Illustration of the effects of interactions on the single-particle spectral function as it would be observed by ARPES. On the left is the case of non-interacting electrons where the single-particle excitations are $\delta$-functions. On the right is the moderately interacting case where electron-electron interactions cause a renormalization of the band dispersion and a finite lifetime. Adapted from [40].

$$
\mathcal{A}(\mathbf{k}, \omega)=Z_{\mathbf{k}} \frac{\Gamma_{\mathbf{k}} / \pi}{\left(\omega-\epsilon_{\mathbf{k}}\right)^{2}+\Gamma_{\mathbf{k}}^{2}}+\mathcal{A}_{i n c}
$$

giving a familiar Lorentzian form for the first term, the "coherent" part of the self-energy, $\mathcal{A}_{c o h}$. We use renormalized values which are defined only in the vicinity of the pole: $\epsilon_{\mathbf{k}}=Z_{\mathbf{k}}\left(\varepsilon_{\mathbf{k}}+\Sigma^{\prime}\right)$ and $\Gamma_{\mathbf{k}}=Z_{\mathbf{k}}\left|\Sigma^{\prime \prime}\right|$. This quasiparticle description is only valid in the proximity close to $\mathrm{E}_{\mathrm{F}}$ and where $\left|\Sigma^{\prime \prime}\right| \ll \epsilon_{\mathbf{k}}-\mu$, that is the level width is narrower than the level spacing. Another important point is to notice the $\mathcal{A}(\mathbf{k}, \omega)$ is broken into two separate terms. The first is the coherent part of the spectral function, corresponding to the quasiparticle pole. This is the first term in Equation 2.16 which corresponds to the part of the spectral function which can be linearized in the vicinity of the quasiparticle pole, $E_{\mathbf{k}}^{*}$. However, the total spectral weight in the coherent part is simply $Z_{\mathbf{k}}$, while the total integrated spectral weight for $\mathcal{A}(\mathbf{k}, \omega)$ must satisfy the sum rule such that 


$$
\int_{-\infty}^{+\infty} \mathcal{A}(\mathbf{k}, \omega) d \omega=1
$$

This is a trivial statement, since $\mathcal{A}(\mathbf{k}, \omega)$ is simply a probability amplitude for a single electron, and the total electron must be conserved! However, it is important to note that this sum rule is difficult to apply in practice, because all unoccupied spectral weight must also be summed over, and this cannot be done without combining ARPES and inverse photoemission (although an approximate sum rule was proposed by Randeria et al. [87]). Thus if the coherent weight is $Z_{\mathbf{k}}$, then the second term in Equation 2.16, $\mathcal{A}_{\text {inc }}$, must encompass $1-Z_{\mathbf{k}}$ of the spectral weight to fulfill the sum rule. In the examples that are discussing, we typically assume that $Z_{\mathrm{k}}$ may be typically between $0.1-1$. Many of the typical approaches to analyzing data is to use a "large Z" phenomenology, where one tracks a peak in the spectra and corresponds that to the quasiparticle pole, and therefore the position of the renormalized band. In Chapters 4 and 5, we will discuss the situation where $Z \rightarrow 0$ due strong electronboson interactions. We should note that although the sum rule for $\mathcal{A}(\mathbf{k}, \omega)$ cannot be fulfilled using ARPES alone, one can address the Luttinger sum rule [16] using ARPES where

$$
\int_{-\infty}^{+\infty} \mathcal{A}(\mathbf{k}, \omega) f(\omega) d \omega=n(\mathbf{k})
$$

relating the momentum distribution function $n(\mathbf{k})$ to the integral of $\mathcal{A}(\mathbf{k}, \omega)$ up to $\mathrm{E}_{\mathrm{F}}$. Since the integral is cut off by the Fermi function, this negates the need for inverse photoemission. If there is some finite quasiparticle weight, $Z_{\mathbf{k}}$, this will manifest itself as a discontinuity in $n(\mathbf{k})$ of magnitude $Z_{\mathbf{k}}$ at $\mathbf{k}_{\mathrm{F}}$. Even in the case of non-Fermi liquid systems, such as Luttinger liquids, where $Z_{\mathbf{k}}=0$ because the oneelectron excitations are unstable against decay into spinons and holons (topological excitations in the spin and charge density, much like solitons), there is some hint for the position of the original $\mathbf{k}_{\mathrm{F}}$ in $n(\mathbf{k})$ of the Luttinger liquid. Although there is no discontinuity in $n(\mathbf{k})$, there is a divergence in $d n(\mathbf{k}) / d \mathbf{k}$ at $\mathbf{k}_{\mathrm{F}}$ for the Luttinger liquid at $\mathrm{T}=0$. For this reason, attempts have been made to use the experimentally determined $n(\mathbf{k})$ to determine the position of the Fermi surface [88]. 


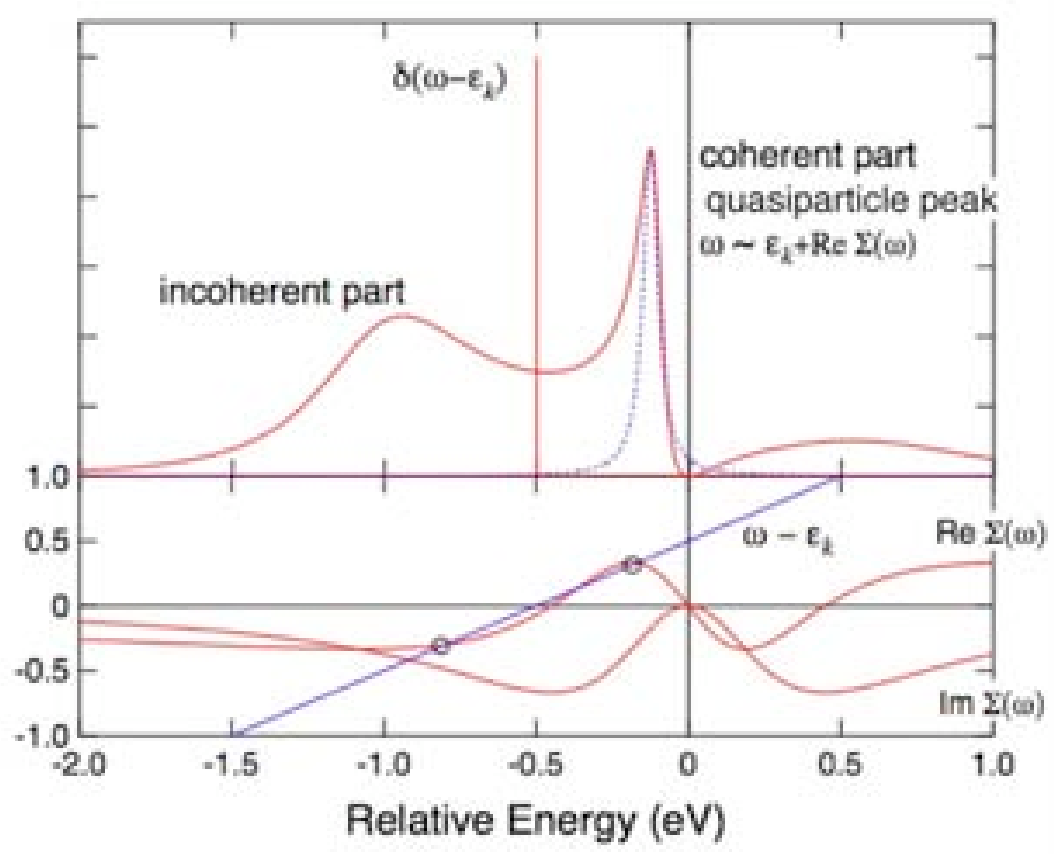

Figure 2.7: Simulation of $\mathcal{A}(\mathbf{k}, \omega)$ using a model self-energy described in the text. The red curve shows $\mathcal{A}(\mathbf{k}, \omega)$, and the blue dashed line shows the coherent quasiparticle peak which was linearly expanded around $E_{\mathbf{k}}^{*}$. The remaining spectral weight represents the incoherent part of the spectral weight. The $\delta$-function shows the position of the unrenormalized bare pole. At the bottom of the figure are the real and imaginary parts of the self-energy, as well the intersections of $\omega-\varepsilon_{0}-\Sigma^{\prime}(\omega)$ (from [89]).

An illustration of the coherent and incoherent pole structure is shown in Figure 2.7, adapted from [89]. The form of the self-energy used in this simulation is $\Sigma(\omega)=$ $\frac{G \omega}{(\omega+i \gamma)^{2}}$ and the coherent and incoherent parts of the spectral function are shown at the top, while $\Sigma^{\prime}(\omega)$ and $\Sigma^{\prime \prime}(\omega)$. 


\subsection{Interpretation and Data Analysis of ARPES Spectra}

\subsubsection{EDC and MDC Analysis}

As discussed will be discussed below, the ARPES spectra were traditionally collected in a "point-by-point" fashion in momentum space using electron analyzers with pinhole apertures. This typically meant a sparse sampling in $\mathbf{k}$-space, so that the primary analysis was performed by analyzing the individual energy distribution curves (EDCs). However, with the latest generation of $2 \mathrm{D}$ multiplexing analyzers, modern ARPES spectra now have a high sampling density in both energy and momentum-space, so one can effectively slice the two-dimensional image into different one-dimensional strips. A slice showing the photoelectron intensity at a fixed $\mathbf{k}$ (actually, fixed $\theta$ ) as a function of $\omega$ is the traditional EDC. Conversely, the photoelectron intensity plotted at a fixed $\omega$ as a function of $\mathbf{k}$ is called a "momentum distribution curve", or MDC.

In some sense, looking at a single EDC gives a better global overview of the spectral function, in that one can observe whether a well-defined quasiparticle peak can be observed, and the distribution of incoherent and coherent spectral weight. Also, tracking subtle features, such as small, low-energy peaks or the presence of gaps should generally be better achieved through EDC analysis. On the other hand, the MDC method has certain specific advantages over the EDC method, especially in the automated quantitative analysis of large data sets, whereas the EDC method tends to be much more subjective and time-consuming. One major advantage of the MDC method is that it largely eliminates effects which are isotropic or very weakly k-dependent. This includes things like the Fermi function, a momentum-independent background, and photoelectron matrix elements (if one is looking at MDC features whose $\Delta \mathbf{k}$ is much smaller than the $\mathbf{k}$ regions where the matrix elements vary). As mentioned above, although a single EDC would tend to carry more interesting information than a single MDC, the simple lineshape of the single MDC make it amenable to computer analysis. The reason for is that the MDC can be approximated 


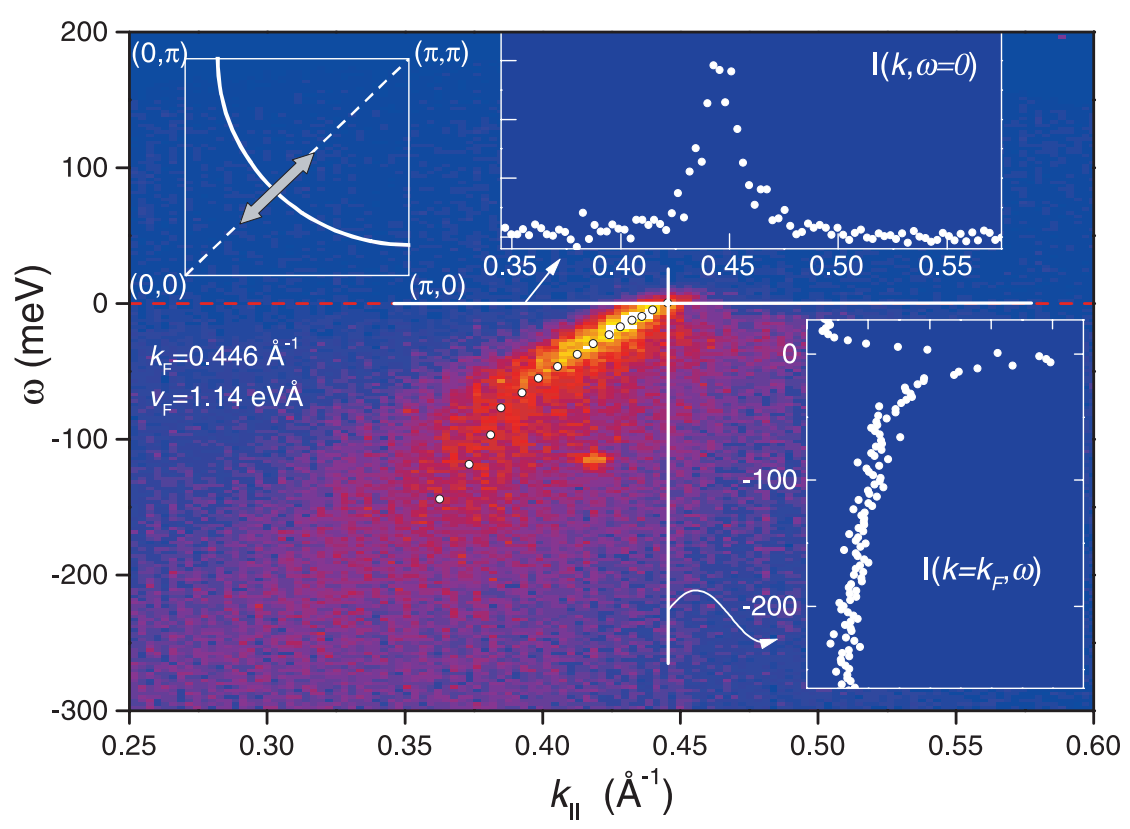

Figure 2.8: Example spectra from $\mathrm{Bi}_{2} \mathrm{Sr}_{2} \mathrm{CaCu}_{2} \mathrm{O}_{8+\delta}$ along the nodal direction demonstrating the concept of EDCs and MDCs. Figure taken from [57], which is believed to the first published use of MDC analysis from a 2D Scienta angle mode image. The $\mathrm{MDC}$ cut at $\mathrm{E}_{\mathrm{F}}$ is shown at the top, while the EDC cut at $\mathbf{k}_{\mathrm{F}}$ is shown at the right.

as a simple Lorentzian if one assumes that the band is approximately linear and the momentum dependence of the self-energy $\Sigma(\mathbf{k}, \omega)$ varies only weakly over the $\Delta \mathbf{k}$ of the MDC. In fact, it appears that this Lorentzian expectation is surprisingly robust in many materials, even strongly correlated compounds such as the high- $\mathrm{T}_{\mathrm{c}} \mathrm{s}$.

The observation and quantification of a kink or break in the MDC dispersion along the nodal direction $[57,58,59]$ was made possible by the use of MDC analysis. Since then, dispersion anomalies arising from electron-boson coupling has been a topic of great interest in a wide variety of materials. The origin of the electron-boson coupling in the cuprates is still a matter of great debate as to whether the boson is a spin excitation (perhaps associated with the famous $\mathbf{q}=(\pi, \pi)$ magnetic resonance), or a lattice phonon, or whether there are spectral signatures of both interactions. The original inspiration for associating the observed behavior to electron-boson interactions was ARPES studies of the Be(0001) surface by LaShell [90] and Hengsberger [91], as shown in Figure 2.9. This data is quite strongly suggestive of the two-pole 


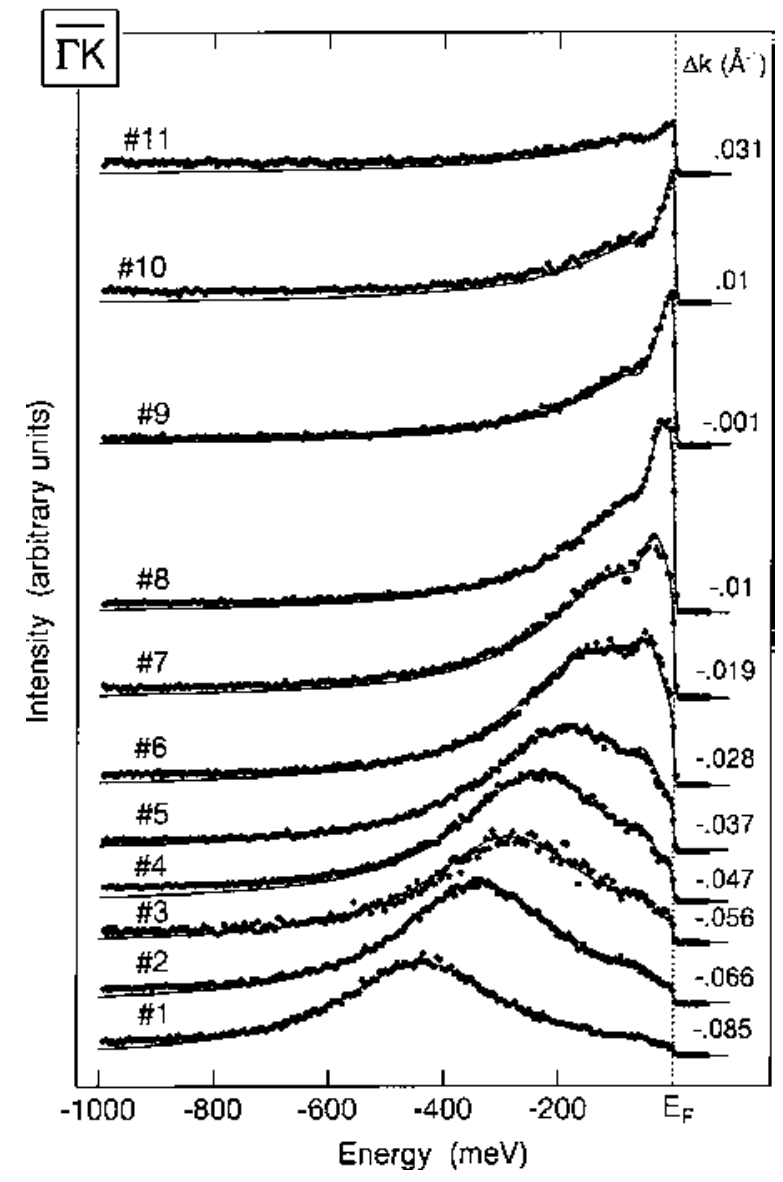

Figure 2.9: EDCs from Be(0001) from Hengsberger et al. [91] showing an unusual, non-Fermi liquid-like lineshape representative of electron-phonon coupling.

calculations of Engelsberg and Schrieffer [92], shown in Figure 2.10, where the lowenergy branch is the heavily phonon-dressed, massive polaronic quasiparticle, and the higher energy branch represents the electron plus one (or more) real phonons emitted.

Very recently, attempts have been made to refine this electron-boson analysis beyond simplistic Debye or Einstein models. In particular, there is typically some fine structure observed in the dispersion (i.e. in $\Sigma^{\prime}$ ) beyond what would expect from a smooth distribution of oscillators (Debye) or a single non-dispersive mode (Einstein). In any real solid, there should be numerous phonon modes which interact with the electrons, and therefore it should not be unreasonable to expect that the fine structure 


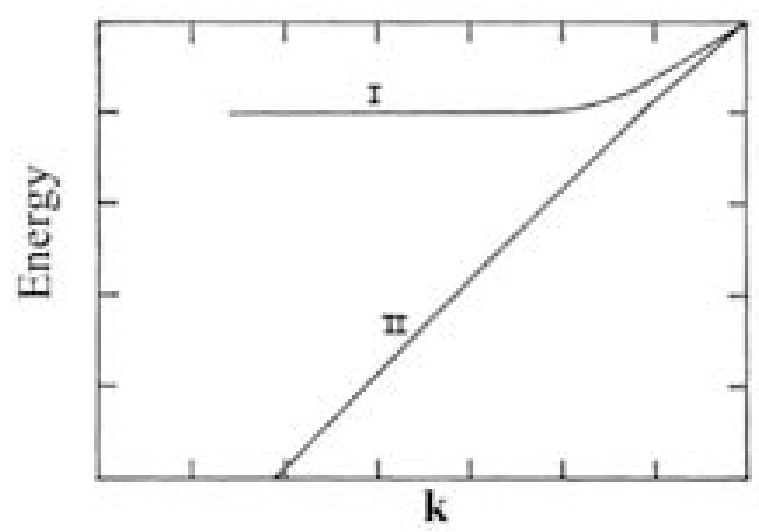

Figure 2.10: Two-pole treatment of electron-phonon interactions from Engelsberg and Schrieffer [92].

observed in the electronic dispersion may arise from the interactions with different phonon modes. Taking a similar approach to what was done in the case of phonon anomalies in the tunneling spectra of the BCS superconductors [93, 94], attempts have been made to extract the Eliashberg function $\alpha^{2} F(\omega ; \varepsilon, \mathbf{k})$ [95], which represents the coupling of the phonon modes to the electrons in the system and therefore provides full information about the electron-phonon interaction. $\alpha^{2} F(\omega ; \varepsilon, \mathbf{k})$ can be related to $\Sigma^{\prime}(\mathbf{k}, \omega)$ through an integral relation

$$
\Sigma^{\prime}(\mathbf{k}, \omega ; T)=\int_{0}^{\infty} \alpha^{2} F(\omega ; \varepsilon, \mathbf{k}) K\left(\frac{\varepsilon}{k T}, \frac{\omega}{k T}\right) d \omega
$$

where $K\left(y, y^{\prime}\right)=\int_{-\infty}^{+\infty} f(x-y) 2 y^{\prime} /\left(x^{2}-y^{\prime 2}\right) d x$, where $f(x)$ is the Fermi distribution function. Because the Eliashberg function is the kernel of this integral, one must perform an integral inversion of $\Sigma^{\prime}(\mathbf{k}, \omega)$ to extract $\alpha^{2} F(\omega ; \varepsilon, \mathbf{k})$, which is extremely challenging. This has been attempted first on the $\operatorname{Be}(10 \overline{10})$ surface [96], and later on $\mathrm{La}_{2-x} \mathrm{Sr}_{x} \mathrm{CuO}_{4}$ [97] using a "maximum entropy method" algorithm to extract $\alpha^{2} F(\omega ; \varepsilon, \mathbf{k})$. This approach is rather ambitious and still very new, and while promising, it remains to be seen how robust and exact this procedure is. However, one thing about this is certain: in order to implement such sophisticated analysis schemes, improvements in the data quality both in terms of the resolution and signal-to-noise ratio will be critical. 


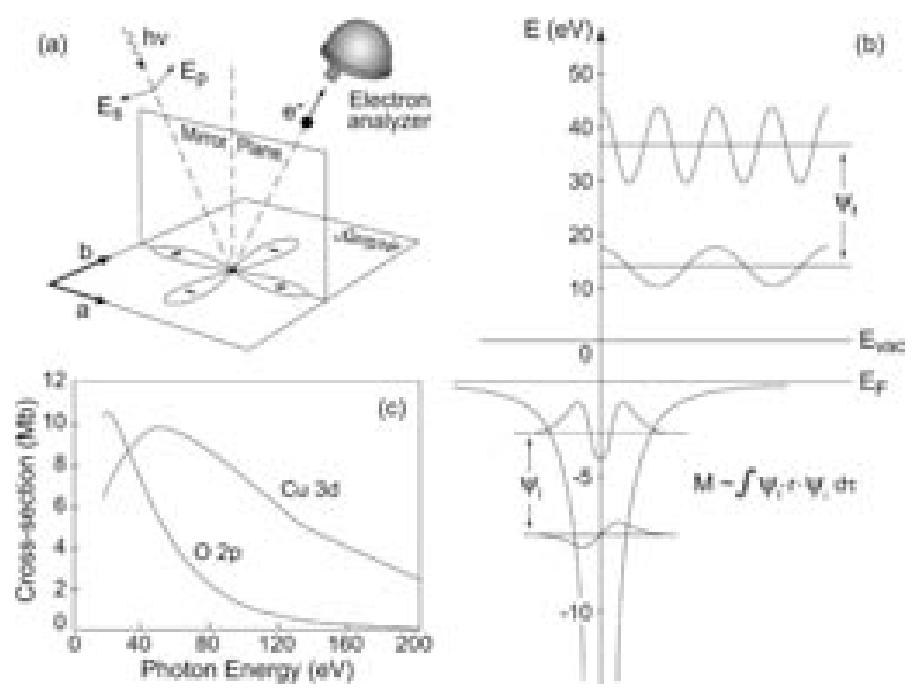

Figure 2.11: Symmetry and parity considerations in the photoelectron matrix element. From [40].

\subsubsection{Matrix Element Effects}

Finally, we come to the issue of the photoelectron matrix elements. Typically the matrix elements are viewed as "one-electron matrix elements", such that the initial and final states can be viewed as a single Slater determinant as in Equation 2.6. In this basic picture and taking the dipole approximation, one can approximate the matrix element as

$$
\left|M_{f, i}^{\mathbf{k}}\right|^{2} \propto\left|\left\langle\phi_{f}^{\mathbf{k}}|\epsilon \cdot \mathbf{x}| \phi_{i}^{\mathbf{k}}\right\rangle\right|^{2}
$$

where we use the commutation relation that $\mathbf{p}=-i m / \hbar[\mathbf{x}, H]$ where $\epsilon$ is the polarization unit vector. Regardless of the details of the initial state wavefunction, one can make simple arguments as to whether emission is allowed or forbidden from basic symmetry and parity arguments. This is shown in Figure 2.11. Because the total integrand must be even for the matrix element to be finite, we can consider for a generic initial state, the case must be such that 


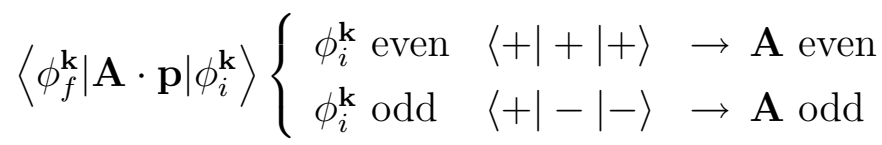

This provides a very simple overview of the matrix elements in the sense of simply discussing the dipole allowed transitions. However, to understand the photoelectron matrix elements in a more detailed way is difficult, as this requires knowledge of the initial states. One approach is to use initial states derived from band theory, and assume the outgoing final states are simply plane waves. At low kinetic energies, using a free-electron plane wave is not the best approach since at low energies the effects of the lattice potential can be sizable even in the cuprates, as shown by studies using very low energy electron diffraction (VLEED) [98].

The variation of the photoelectron matrix element with photon energy in real materials is not something that can be understood particularly easily. For instance, it is generally known that $\mathrm{Ca}_{2-x} \mathrm{Na}_{x} \mathrm{CuO}_{2} \mathrm{Cl}_{2}, \mathrm{La}_{2-x} \mathrm{Sr}_{x} \mathrm{CuO}_{4}, \mathrm{Bi}_{2} \mathrm{Sr}_{2} \mathrm{CaCu}_{2} \mathrm{O}_{8+\delta}$, and $\mathrm{Nd}_{2-x} \mathrm{Ce}_{x} \mathrm{CuO}_{4}$ all have very different photoelectron matrix elements despite the fact that the basic building block - the $\mathrm{CuO}_{2}$ plane - is common to all the cuprates. Therefore, the photoelectron matrix element depends very sensitively on the precise details of the crystal structure, and one cannot simply consider the $\mathrm{Cu} 3 d$ and $\mathrm{O}$ $2 p$ states in the $\mathrm{CuO}_{2}$ plane for a realistic description of the photoelectron matrix elements. In fact, attempts have been made to describe the photoelectron matrix elements of $\mathrm{Sr}_{2} \mathrm{CuO}_{2} \mathrm{Cl}_{2}[99,100]$, but have only been based on fairly simple models. Very detailed one-step calculations of the matrix elements in $\mathrm{Bi}_{2} \mathrm{Sr}_{2} \mathrm{CaCu}_{2} \mathrm{O}_{8+\delta}$ have been performed $[77,78]$ and shows that the photoelectron matrix element is extremely sensitive to only small changes in the incident photon energy. 


\subsection{Experimental Details of the ARPES System at SSRL Beamline 5-4}

Virtually all results reported in this thesis were performed at Normal Incidence Monochromator (NIM) Beamline 5-4 of the Stanford Synchrotron Radiation Laboratory (SSRL). At the time of these measurements, SSRL was using the SPEAR2 ring (a second-and-a-half generation synchrotron) with operating characteristics of $3.0 \mathrm{GeV}$ and $100 \mathrm{~mA}$. Beamline 5 had an undulator insertion device, so the photon flux was rather respectable $\left(\sim 10^{11} / \mathrm{s} \mathrm{mm}^{2}\right)$, although certainly not on par with lower energy, third-generation sources such as the Advanced Light Source at Lawrence Berkeley Laboratory. The photon spot size of our measurements was typically on the order of $(\sim 0.5 \times 0.3 \mathrm{~mm})$ which was generally acceptable for most samples, since handling crystals much smaller than $1 \times 1 \mathrm{~mm}$ becomes increasingly difficult. One of the powerful aspects of BL 5-4 was the NIM, which allowed for highly monochromatic photons with a resolving power of $\sim 10^{4}$, which meant $\Delta E_{h \nu} \sim 2-3 \mathrm{meV}$. However, the geometry of the NIM and low reflectivity of gratings in this configuration restricted our work to between $h \nu=13-32 \mathrm{eV}$; above $32 \mathrm{eV}$, the grating efficiency drops precipitously. On the other hand, grazing incidence monochromators (such as spherical grating monochromators, SGMs, or plane grating monochromators, PGMs) can cover a wide range in $h \nu$, but generally do not have the high resolving power of the NIMs. In addition, NIMs strongly suppress undesired higher order photons which can lead to an additional background of photoelectrons, while grazing incidence monochromators produce a much higher spectrum of higher harmonic light. These considerations made BL 5-4 an ideal facility for very high resolution, synchrotron-based ARPES measurements. A picture of the beamline is shown in Figure 2.12a.

\subsubsection{Scienta Electron Analyzer and Experimental Endstation}

ARPES experienced a renaissance in the very late 1990's and early 2000's with the advent of 2D multiplexing analyzers by the Swedish company Gammadata Scienta 
a)

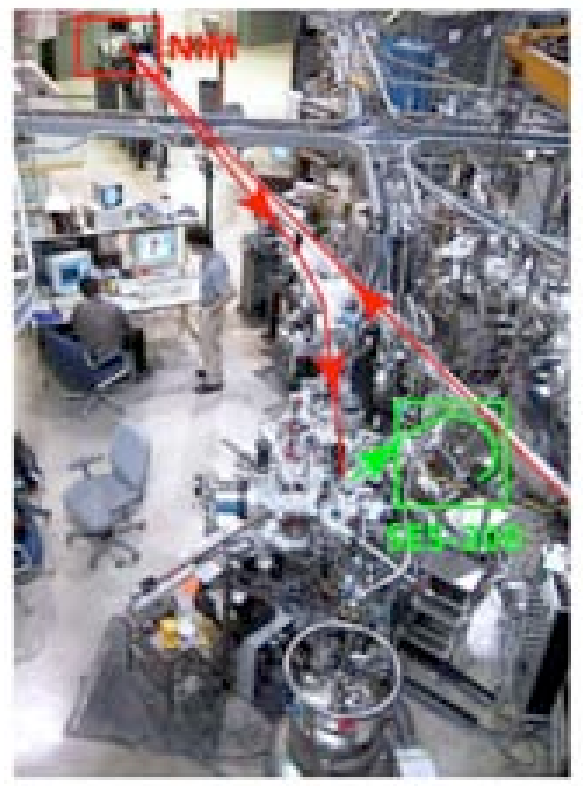

b)

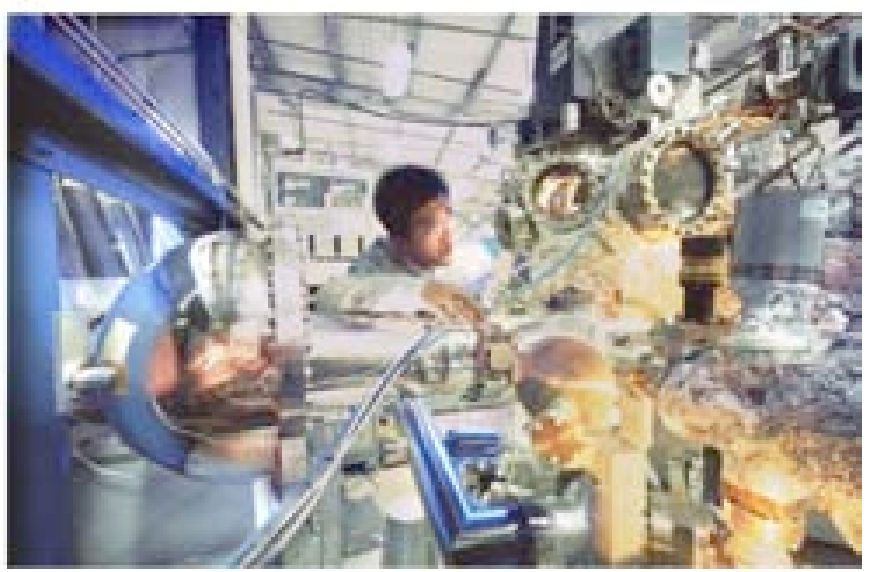

Figure 2.12: (a) View of the Stanford Synchrotron Radiation Laboratory Normal Incidence Monochromator Beamline 5-4. Red lines indicate the beam trajectory, and green lines indicate the photoelectron trajectories. The NIM and analyzer are clearly marked. (b) Closeup view of the 5-4 ARPES endstation. The hemispherical SES-200 analyzer is on the left, and the entrance beam pipe, upper chamber, and manipulator are shown at the right. Beamline Jesus sold separately.

which could measure a range of electron kinetic energies and angles simultaneously. The way that traditional electron analyzers worked was that their angular acquisition was based on a small pinhole aperture. The position of the pinhole relative to the sample normal determined the photoelectron $\mathbf{k}$, and the solid angle subtended by the pinhole determined $\Delta \mathbf{k}$. The problem with this approach is that all electrons outside this pinhole are discarded, and only one wavevector can be measured at a time. The great advantage of the Scienta analyzers is that the electron lens system for these analyzers allowed for parallel detection of many $(\sim 100)$ angular channels, thereby increasing the measurement efficiency by about two orders of magnitude. The Scienta analyzers can operate in two electrostatic lens modes: "angle" mode, where the analyzer multiplexes as a function of angle, and "transmission" mode, where the analyzer multiplexes in real space (so as to produce a one-dimensional real-space 


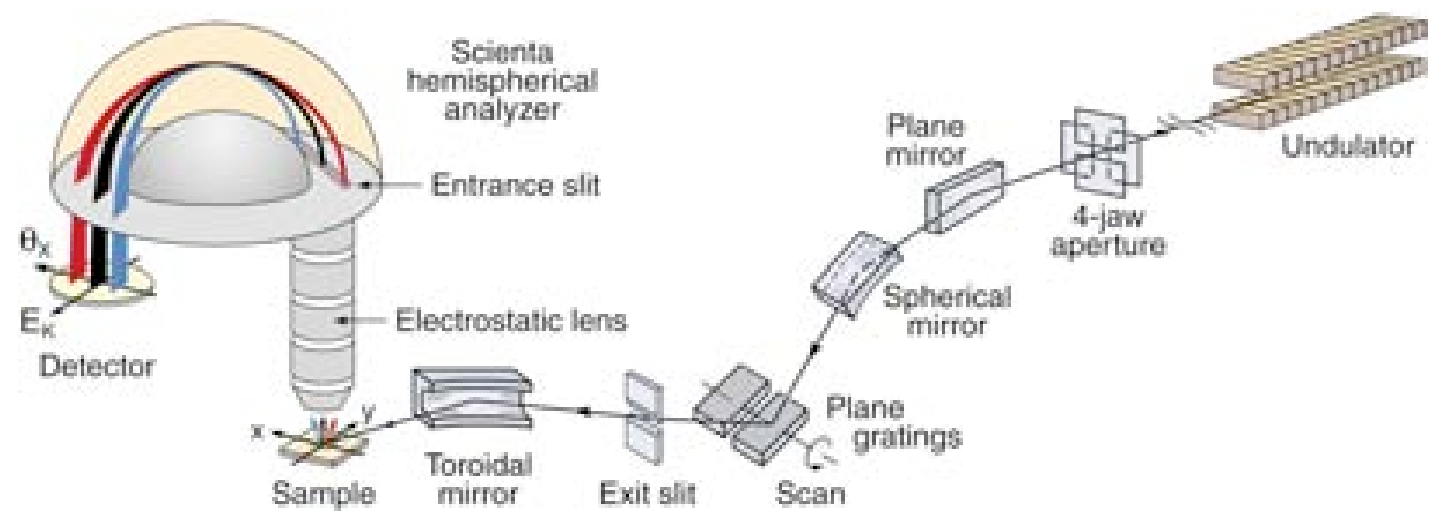

Figure 2.13: Schematic of Scienta analyzer on a beamline. Photoelectrons with different take-off angles are imaged onto the long entrance slit, and pass through the concentric hemispheres, and strike the microchannel plates. Note the beamline in question is not a NIM, but a PGM beamline. From [40].

image of the photoelectrons emitted from the sample). In addition, the most popular Scienta analyzers were rather large, with a radius of $200 \mathrm{~mm}$, also increasing the throughput (which goes as $r^{2}$ ). Illustrations of such improvements in the angular multiplexing and resolution are shown in Figures 2.16 and 2.15. A simple schematic of a Scienta analyzer working at a beamline is shown in Figure 2.13.

A more detailed picture of the $200 \mathrm{~mm}$ Scienta analyzers are shown in Figure 2.14, and a picture of our analyzer in action is shown in Figure 2.12b. In Figure 2.14a, we show a picture of the SES R4000, the latest model of the $200 \mathrm{~mm}$ electron analyzer (at BL 5-4, we are using the SES-200, a very similar but older model). The electrons pass through the entrance aperature, at the far left, through a three-element lens system, through the entrance slits, and then the two concentric hemispheres housed inside the large stainless steel dome. The electrons are finally detected at the bottom of the analyzer, after the electron signals are amplified by a pair of microchannel plates (MCPs) and a phosphor screen. Flashes on the phoshphor screen are then detected ex situ by a CCD camera looking through the window, visible at the bottom flange. In the analyzer orientation shown in Figure 2.14a, the vertical direction on the 2D MCP corresponds to kinetic energy, and the horizontal, the angular channels. In Figure 2.14b, we show an SES-2002 (the HeLM system installed in the basement 

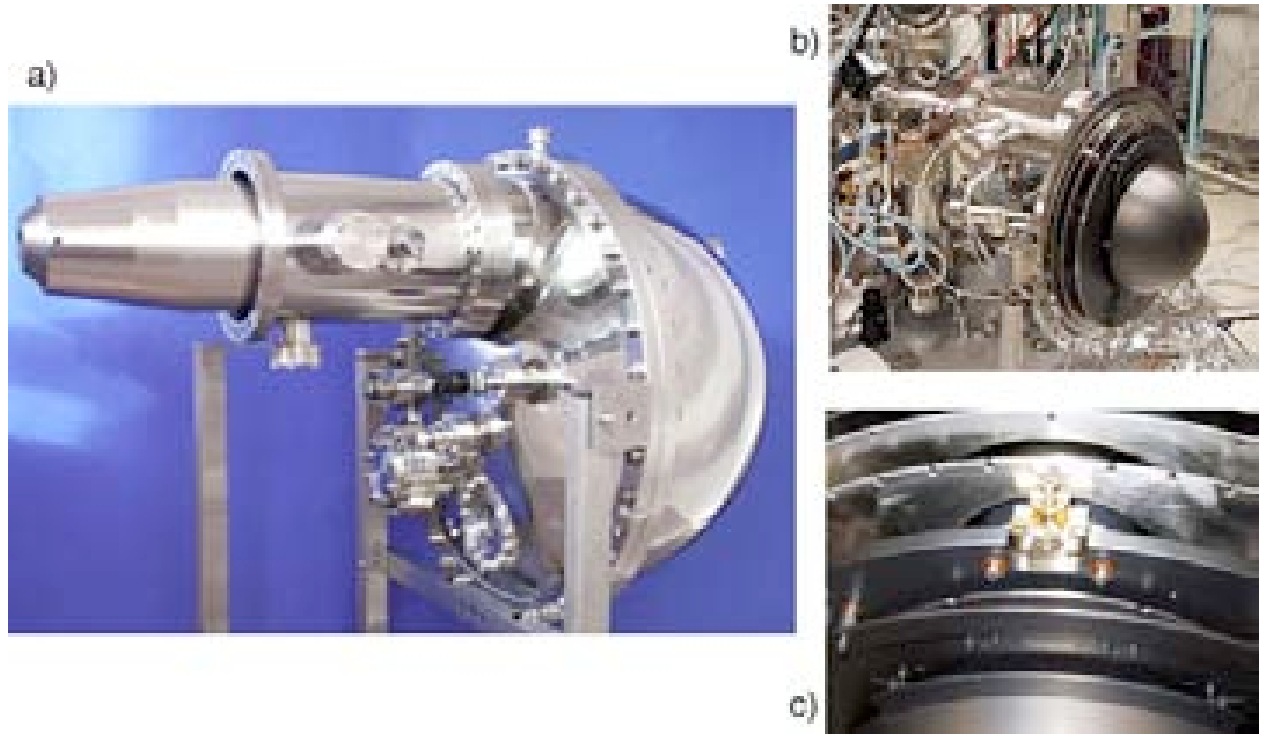

Figure 2.14: (a) Gammadata Scienta SES R4000 electron analyzer. (b) SES-2002 with the steel dome, $\mu$-metal shield, and outer hemisphere removed. (c) Close-up of the SES-2002 entrance slits.

of McCullough) with the stainless steel dome, $\mu$-metal shield, and outer hemisphere removed. In Figure 2.14c, we show a close-up of the electron entrance slit, visible in the bottom center part of the picture. The slits are mounted on a wheel, so that one can select entrance slits of different widths, so as to trade off energy resolution against counting rate, if necessary.

In order cover a wide span in $\mathbf{k}$-space, one needs to change the relative angles between the sample normal and the detector. Ideally, it would be better to move the electron detector, since rotation of the sample will change the polarization of the incoming photons relative to the crystal axes. However, because the $200 \mathrm{~mm}$ analyzers are so large and unwieldy, it is much easier to move the crystal sample. For that purpose, we use a low-temperature, two-axis manipulator shown in Figure 2.17. The manipulator cools the sample stage via $\mathrm{Cu}$ braids to a working temperature of approximately $15 \mathrm{~K}$. The manipulator in Figure 2.17a is the manipulator at BL 5-4, and a cleaved sample is visible at the center of the picture. A CAD drawing of the new low-temperature manipulator on the HeLM system is shown in Figure 2.17b. This manipulator is also now operational and can reach a sample temperature of less 
a)

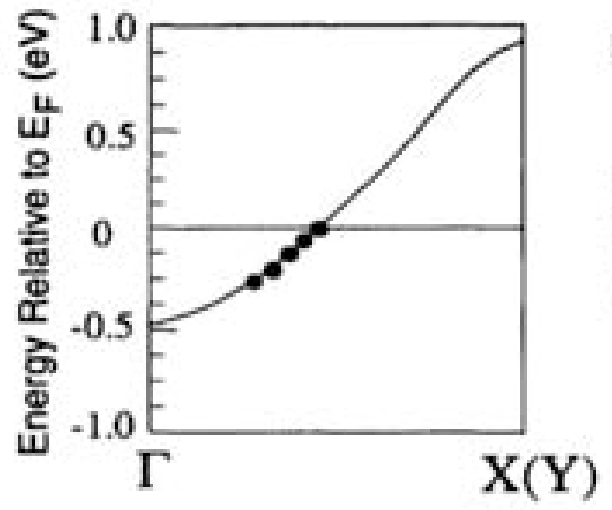

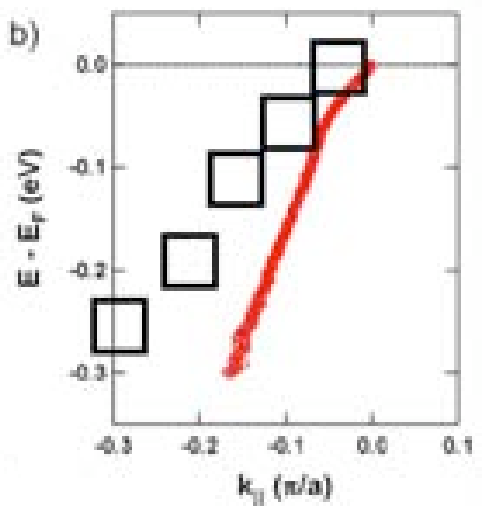

Figure 2.15: Demonstration of improvements in angular acquisition and resolution. (a) Data from $\mathrm{Bi}_{2} \mathrm{Sr}_{2} \mathrm{CaCu}_{2} \mathrm{O}_{8+\delta}$ showing the band dispersion, taken from Dessau et al. [101]. (b) Dispersion from Bogdanov et al. [58] using a multiplexing SES-200 analyzer, showing fine structure (a "kink" at $70 \mathrm{meV}$ ) in the dispersion undetectable with the previous generation of analyzers. Data from (a) are overlaid as open squares.

a)

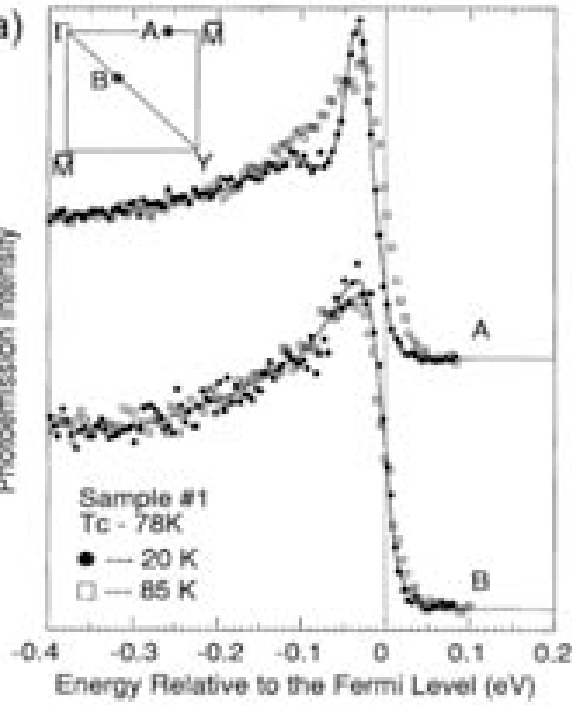

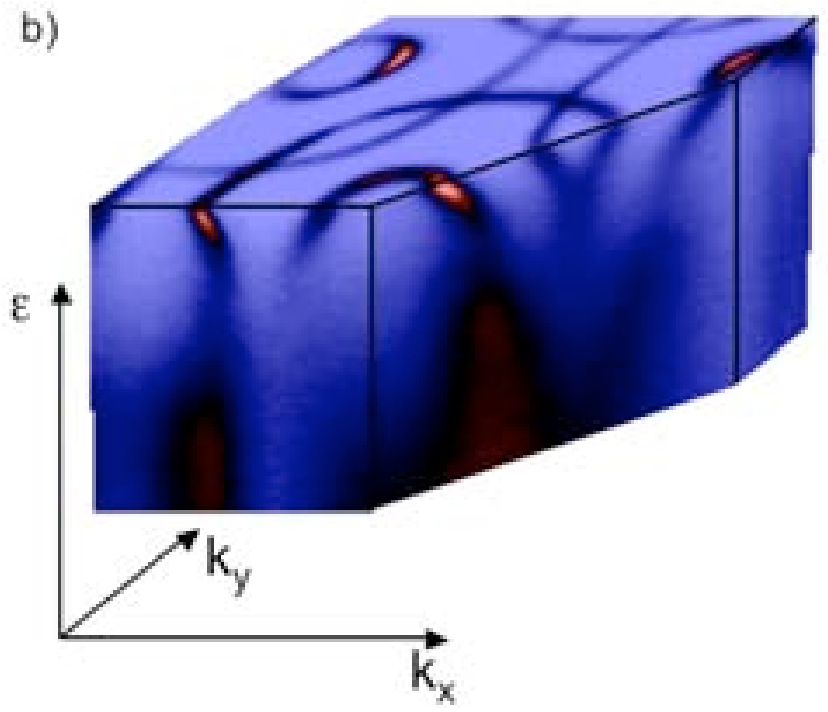

Figure 2.16: Demonstration of advances in data throughput. (a) Data from $\mathrm{Bi}_{2} \mathrm{Sr}_{2} \mathrm{CaCu}_{2} \mathrm{O}_{8+\delta}$ showing the first evidence from ARPES of an anisotropic superconducting gap, from Z.-X. Shen et al. [46]. (b) 3D intensity wedge from $\mathrm{Sr}_{2} \mathrm{RhO}_{4}$ from Baumberger et al. [102]. The 4 data points in (a) took approximately 12 hours to collect, while the $10^{4}+$ data points in (b) took approximately 9 hours. 
a)

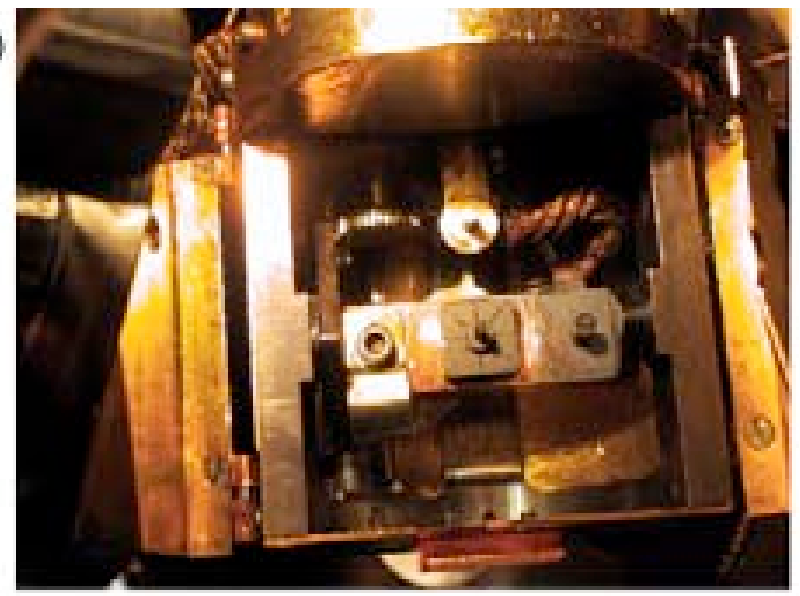

b)

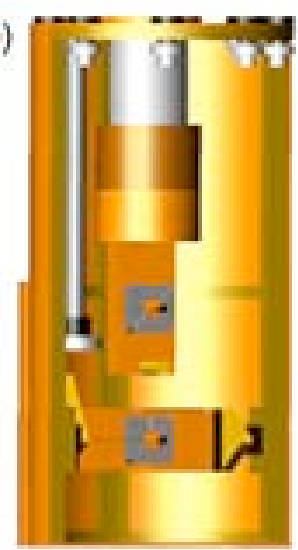

Figure 2.17: (a) Low-temperature sample manipulator at BL 5-4 with cleaved sample. (b) CAD drawing of the HeLM low-temperature manipulator.

than $10 \mathrm{~K}$.

\subsubsection{Resolution and Detector Issues}

Before moving on, we will mention a few technical issues related to the Scienta analyzers. Because these electron analyzers are far more sophisticated than the previous generation of electron analyzers, there are many complications and artifacts. These considerations were considered throughout the data taking and analysis process in this thesis, but will only be touched upon once here. First, these lenses are astigmatic, and the linear proportionality between real space channels and photoelectron angle is only accurate in the center $\left(\sim \pm 5^{\circ}\right)$ part of the detector, where at the edges, the spacing of angles per channel begins to increase. Therefore, we typically only use the center channels of the detector. Secondly, the angular response function is highly irregular (i.e. the detection efficiency varies strongly across channels), and this needs to be corrected for in some manner. Two typical approaches to calibrating against the detector response are to measure the angular response using an isotropic (angle-averaged sample), such as polycrystalline $\mathrm{Au}$, and divide the measured spectra by the angular profile of the Au. The other is to self-normalize the spectra against 
the angular profile within some energy window, for instance, using above- $\mathrm{E}_{\mathrm{F}}$ electrons from second-order light, or some featureless part of the valence band. Not only does the detector response vary with channels, but also the kinetic energy position can vary slightly across the detector in angle mode. In the SES-200 at BL 5-4, this variation is slight $(\sim 3 \mathrm{meV})$ and primarily at the far edges of the detector, and the variation across the center channels is less than 1-2 meV. This is usually corrected for by taking an high resolution, angle-resolved Au spectrum before each measurement, and correcting for the energy "dispersion" across the detector by measuring $\mathrm{E}_{\mathrm{F}}$ at every angular channel. In addition, the response of the detector assembly in the analyzer is highly nonlinear. At high electron count rates, the measured signal increases superlinearly versus photoelectron number before detector saturation, and has been documented in detail by Mannella et al. [103]. To check against this detector nonlinearity, we typically varied the incident photon flux and compared the overall lineshape. Fortunately (or unfortunately), at the low photoelectron yields for the near- $\mathrm{E}_{\mathrm{F}}$ states in the cuprates, detector nonlinearity is hardly a concern.

Due to the surface sensitivity of the ARPES technique, ultrahigh vacuum (UHV) conditions are of the utmost importance. Experiments were typically performed at pressures of better than $5 \times 10^{-11}$ torr, and sample lifetimes were typically on the order of 24 hours or longer. Our manipulator was able to span temperatures from $\sim 15 \mathrm{~K}$ to $400 \mathrm{~K}$; temperatures above room temperature were generally achieved by measuring after a chamber bake and running the sample heater. It was typically found that warming the sample manipulator above $20 \mathrm{~K}$ could cause large pressure bursts of up to $1 \times 10^{-9}$ torr, due to the release of adsorbed gases, sometimes resulting in a severely aged sample surface. This was particularly problematic with samples which had reactive sample surfaces whose termination layer was not van der Waals. Therefore, reliable temperature dependent measurements and temperature cyclings were challenging, and often best performed by cooling from higher temperatures, rather than heating. For low-temperature measurements, however, samples were typically cooled to base temperature, then cleaved in situ, and then realigned by laser reflection. The polarization of the incoming photons was in the horizontal plane (parallel to the floor) and incident about $50^{\circ}$ to the sample normal (electron 
analyzer axis).

Finally, a word about the effects of energy and angular resolution. Because of the nature of the 2D detector, our measured intensity at any point is a product of the energy resolution and the angular resolution. In principle, we should perform a three-dimensional deconvolution $(\omega, \theta, \phi)$. However, within a single 2D image, we do not have information on the angular direction transverse to the detector slit. If we choose the analyzer cut direction such that the bands are dispersive along the cut direction and non-dispersive perpendicular to the cut direction, then we can effectively eliminate the transverse angular resolution. However, in this case, we still need to deal with the energy resolution as well as the angular resolution along the slit direction. This is equivalent to deblurring a picture, a difficult inverse problem which also requires absolute knowledge of the $2 \mathrm{D}$ resolution function. One can also attempt to extract and model the self-energy, while iterating an analysis of the convoluted simulated data until it is consistent with the original experimental data. This is discussed in some detail in [104]. However, before one attempts to extract quantitative values from ARPES spectra (such as quasiparticle lifetimes) or perform very sophisticated analyses of the spectra, a careful accounting of the detector resolution will be essential.

\subsubsection{Transformation from angles to $\mathrm{k}$}

In Figure 2.18, we show a schematic sketch of our experimental setup, and the orientation and angles of the sample with respect to the detector. The sample surface has three degrees of rotation, but only one $(\theta$, the cryostat axis) is fixed in space relative to the detector, while $\phi$, the sample flip stage rotates about $\theta$, and $\alpha$, the azimuthal rotation of the sample surface (or moly piece), rotates about both $\phi$ and $\theta$. Therefore, the exact values of $k_{x}, k_{y}$ and $k_{z}$ depends in a rather complex manner on these angles. In addition, there is an analyzer angle, $\beta$, which is the angle the emitted photoelectron makes with respect to the long detector slit. However, one can treat the general transformation from angle space to real space $(x, y, z)$ by transformation matrices. 


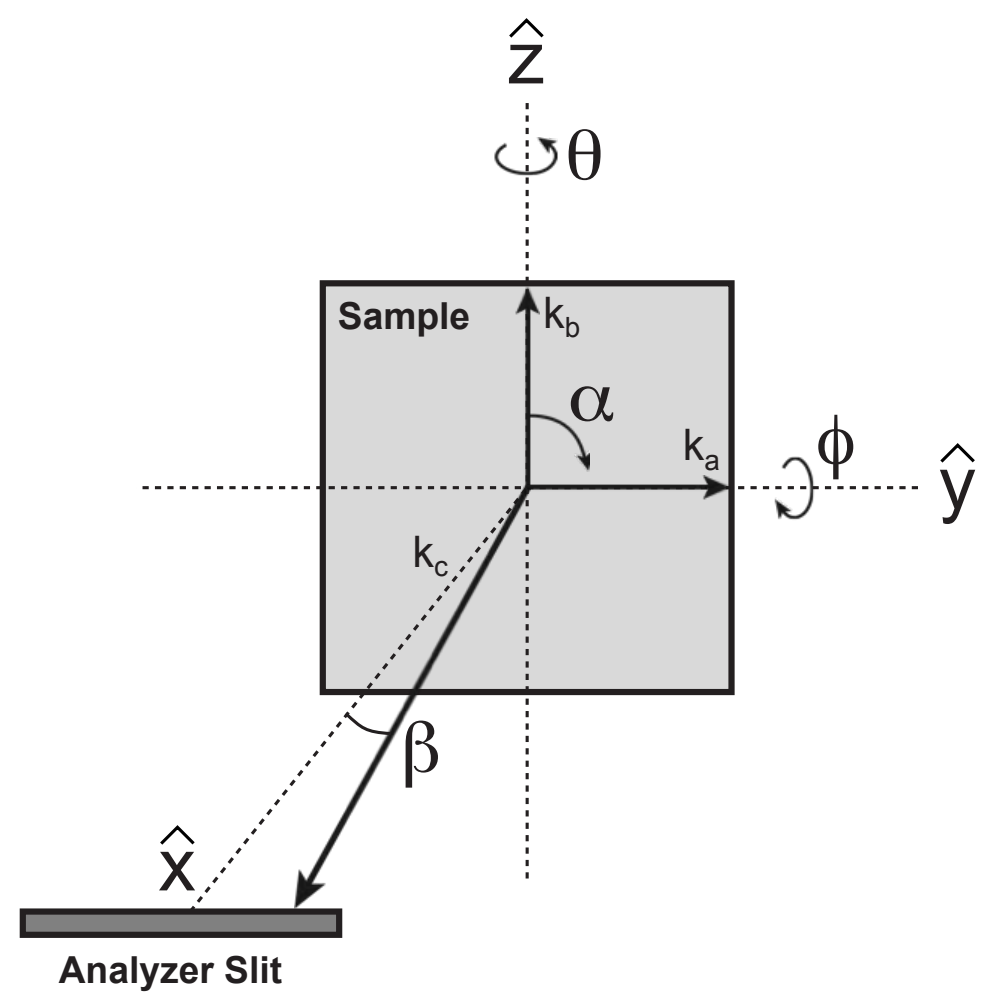

Figure 2.18: Schematic of angles and coordinates of experimental setup (manipulator and analyzer).

$$
\begin{aligned}
& \hat{\theta}=\left[\begin{array}{ccc}
\cos \theta & -\sin \theta & 0 \\
\sin \theta & \cos \theta & 0 \\
0 & 0 & 1
\end{array}\right] \\
& \hat{\phi}=\left[\begin{array}{ccc}
\cos \phi & 0 & -\sin \phi \\
0 & 1 & 0 \\
\sin \phi & 0 & \cos \phi
\end{array}\right] \\
& \hat{\alpha}=\left[\begin{array}{ccc}
1 & 0 & 0 \\
0 & \cos \alpha & \sin \alpha \\
0 & -\sin \alpha & \cos \alpha
\end{array}\right]
\end{aligned}
$$

while the real space and crystal-space axes can be represented as vectors 


$$
\begin{gathered}
\mathbf{R}=\left[\begin{array}{l}
x \\
y \\
z
\end{array}\right] \\
\mathbf{C}=\left[\begin{array}{l}
k_{c} \\
k_{a} \\
k_{b}
\end{array}\right]
\end{gathered}
$$

assuming a tetragonal crystal with $a, b$, and $c$-axes oriented as shown in Figure 2.18 , and we measure outgoing electrons at a given $x, y$, and $z$. Because the $\phi$ axis rotates around $\theta$, and the $\alpha$ axis rotates around both $\phi$ and $\theta$, it is important to operate the $\hat{\theta}, \hat{\phi}$, and $\hat{\alpha}$ operators in the correct order because they will obviously not commute. The order of operation obviously depends on the setup of that particular manipulator. So, in our case, we will have the situation where

$$
\begin{gathered}
\hat{\theta} \hat{\phi} \hat{\alpha} \mathbf{C}=\mathbf{R} \\
\hat{\alpha}^{-1} \hat{\phi}^{-1} \hat{\theta}^{-1} \mathbf{R}=\mathbf{C}
\end{gathered}
$$

We assume that the analyzer and sample are properly aligned such that electrons coming directly off the sample normal strike the center of the analyzer slit. Then, we can also express the incident angle along the parallel detection direction of the analyzer slit as a unit vector, such that $\mathbf{R}=(\cos \beta, 0, \sin \beta)$ if the slit is vertical (along the $z$ axis), or horizontal $(\cos \beta, \sin \beta, 0)$ along the $y$ axis. We will leave the actual matrix elements as an exercise for the reader, but in general (where $s$ and $c$ are shorthand for sin and cos),

$$
\hat{\alpha}^{-1} \hat{\phi}^{-1} \hat{\theta}^{-1}=\left[\begin{array}{ccc}
c \theta c \phi & c \phi s \theta & s \phi \\
s \alpha s \phi c \theta-s \theta c \alpha & s \alpha s \theta s \phi+c \alpha c \theta & -s \alpha c \phi \\
-s \alpha s \theta-c \alpha s \phi c \theta & s \alpha c \theta-c \alpha s \phi s \theta & c \alpha c \theta
\end{array}\right]
$$




\section{Chapter 3}

\section{Properties of $\mathrm{Ca}_{2-x} \mathrm{Na}_{x} \mathrm{CuO}_{2} \mathrm{Cl}_{2}$}

The field of high-temperature superconductivity is one driven strongly by advances in materials science. Due to its favorable cleaving characteristics, $\mathrm{Bi}_{2} \mathrm{Sr}_{2} \mathrm{CaCu}_{2} \mathrm{O}_{8+\delta}$ has been the workhorse material for surface-sensitive techniques such as ARPES and STM for the past decade. However, $\mathrm{Bi}_{2} \mathrm{Sr}_{2} \mathrm{CaCu}_{2} \mathrm{O}_{8+\delta}$ has a number of complications associated with its crystal structure and chemistry. First, $\mathrm{Bi}_{2} \mathrm{Sr}_{2} \mathrm{CaCu}_{2} \mathrm{O}_{8+\delta}$ cannot typically be grown down to low hole dopings and is usually restricted in doping level to a vicinity around the top of the superconducting dome. Very recently, $\mathrm{Bi}_{2} \mathrm{Sr}_{2} \mathrm{CaCu}_{2} \mathrm{O}_{8+\delta}$ has been pushed down to non-superconducting compositions by La substitution into the Sr site [105], and ARPES measurements have been performed $[106,107]$, although the chemical quality of these samples has not yet been verified in detail. In addition, the precise doping level is difficult to ascertain because of the oxygen non-stoichiometry, $\delta$. The doping level is often calibrated versus $\mathrm{T}_{\mathrm{c}}$, but in fact, this is a rather poor metric since many other factors can strongly affect $T_{c}$, such as Bi-Sr cross-substitution or cation disorder [108]. In addition, the bilayer band splitting in $\mathrm{Bi}_{2} \mathrm{Sr}_{2} \mathrm{CaCu}_{2} \mathrm{O}_{8+\delta}$ causes severe complications in the determination of the electronic structure, as recently demonstrated by many studies $[109,110,111]$. Finally, structural complications such as the incommensurate superstructure modulation in the Bi-O plane and orthorhombic distortions result in an extremely complicated unit cell which does not resemble the simple square $\mathrm{CuO}_{2}$ plane. As a result, $\mathrm{Ca}_{2-x} \mathrm{Na}_{x} \mathrm{CuO}_{2} \mathrm{Cl}_{2}$ is a far cleaner system in which to study the Mott insulator to 


\begin{tabular}{ccccc}
\hline \hline $\mathrm{T}(\mathrm{K})$ & $a(\AA)$ & $c(\AA)$ & $Z_{\mathrm{Ca}}$ & $Z_{\mathrm{Cl}}$ \\
\hline 10 & $3.8673(5)$ & $14.9567(25)$ & $0.39549(40)$ & $0.18325(20)$ \\
300 & $3.8745(4)$ & $15.0758(16)$ & $0.39644(24)$ & $0.18222(12)$ \\
\hline \hline
\end{tabular}

Table 3.1: Lattice constants and structural parameters of $\mathrm{Ca}_{2} \mathrm{CuO}_{2} \mathrm{Cl}_{2}$ at $10 \mathrm{~K}$ and $300 \mathrm{~K}$ obtained from Rietveld analysis of powder neutron diffraction in [112]. $\mathrm{Cu}$ is at the origin $(0,0,0), \mathrm{O}(0,0.5,0)$, and $\mathrm{Ca}$ and $\mathrm{Cl}$ at $(0,0, Z)$

superconductor evolution in the cuprates, and the properties of $\mathrm{Ca}_{2-x} \mathrm{Na}_{x} \mathrm{CuO}_{2} \mathrm{Cl}_{2}$ will be detailed in the following chapter.

\subsection{Physical Properties of $\mathrm{Ca}_{2-x} \mathrm{Na}_{x} \mathrm{CuO}_{2} \mathrm{Cl}_{2}$}

$\mathrm{Ca}_{2-x} \mathrm{Na}_{x} \mathrm{CuO}_{2} \mathrm{Cl}_{2}$ crystallizes in the canonical $\mathrm{K}_{2} \mathrm{NiF}_{4}$ (or "214") single-layer perovskite structure which is the $n=1$ sequence of the Ruddlesden-Popper series $\mathrm{A}_{n+1} \mathrm{M}_{n} \mathrm{O}_{3 n+1}$. Unlike many cuprates, $\mathrm{Ca}_{2-x} \mathrm{Na}_{x} \mathrm{CuO}_{2} \mathrm{Cl}_{2}$ retains a tetragonal $I 4 / \mathrm{mmm}$ symmetry $\left(a=b, \theta=90^{\circ}\right)$ for all temperatures and doping levels currently studied, and therefore should provide an excellent realization of the undistorted, square $\mathrm{CuO}_{2}$ plane. The primary difference between $\mathrm{Ca}_{2-x} \mathrm{Na}_{x} \mathrm{CuO}_{2} \mathrm{Cl}_{2}$ and other cuprates is that the apical site above the planar $\mathrm{Cu}$ in the $\mathrm{CuO}_{2}$ plane is a $\mathrm{Cl}^{-}$, instead of the typical $\mathrm{O}^{2-}$. However, the main physical properties of $\mathrm{Ca}_{2-x} \mathrm{Na}_{x} \mathrm{CuO}_{2} \mathrm{Cl}_{2}$ do not appear grossly different from those of other cuprates which do have apical oxygen sites. The most detailed structural study of this family was performed using single-crystal neutron scattering on undoped $\mathrm{Ca}_{2} \mathrm{CuO}_{2} \mathrm{Cl}_{2}$ by Vaknin et al. [112]. The crystal structure at $10 \mathrm{~K}$ and $300 \mathrm{~K}$ is summarized in Table 3.1, obtained from Rietveld analysis of powder neutron diffraction in [112]. $\mathrm{Cu}$ is at the origin $(0,0,0)$, $\mathrm{O}(0,0.5,0)$, and $\mathrm{Ca}$ and $\mathrm{Cl}$ at $(0,0, Z)$. These measurements also give a Néel temperature for $\mathrm{Ca}_{2} \mathrm{CuO}_{2} \mathrm{Cl}_{2}$ as $247 \pm 5 \mathrm{~K}$. Raman scattering measurements on the similar sister compound, $\mathrm{Sr}_{2} \mathrm{CuO}_{2} \mathrm{Cl}_{2}$ give an estimate for $J \sim 125 \mathrm{meV}$ [113].

Large single crystals of pure $\mathrm{Ca}_{2} \mathrm{CuO}_{2} \mathrm{Cl}_{2}$ can be grown from a slow cooling a molten flux of $60 \% \mathrm{Ca}_{2} \mathrm{CuO}_{2} \mathrm{Cl}_{2}$ and $40 \% \mathrm{CaCl}_{2}$ from $900^{\circ}$ in a alumina crucible, as 

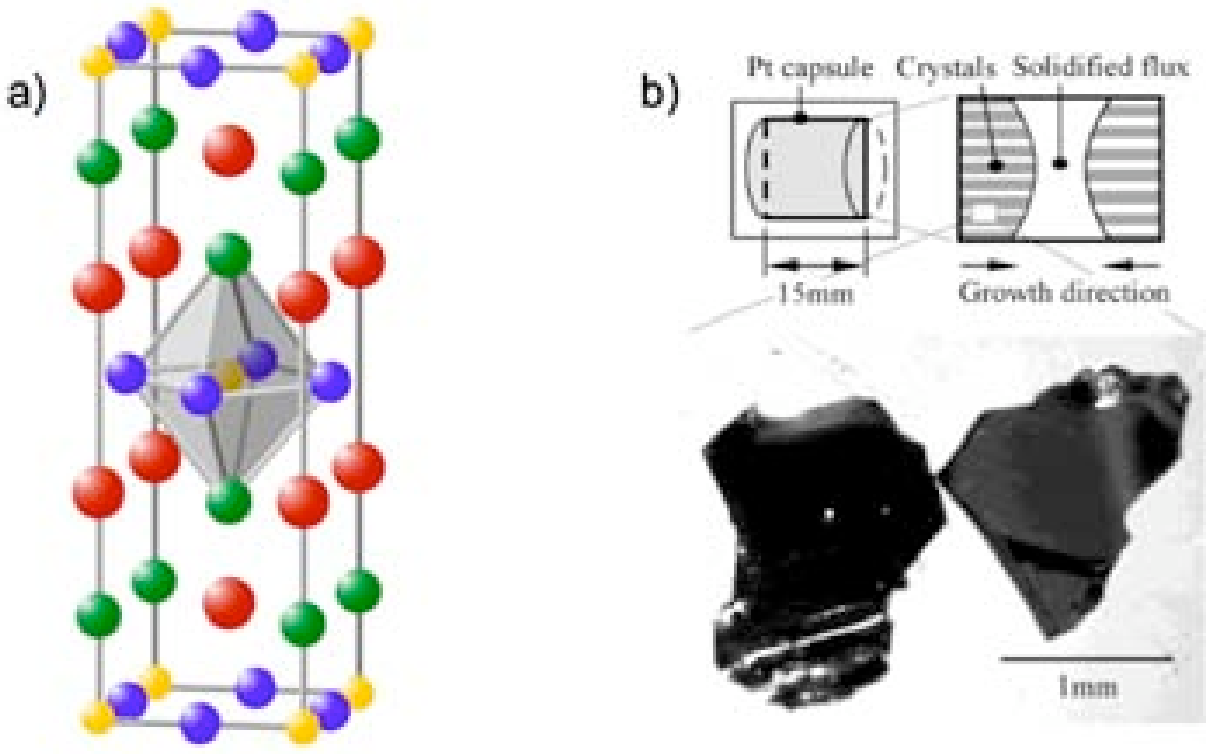

Figure 3.1: (a) Structure of $\mathrm{Ca}_{2-x} \mathrm{Na}_{x} \mathrm{CuO}_{2} \mathrm{Cl}_{2}$. The primitive unit cell is bodycentered tetragonal and has an $I 4 / \mathrm{mmm}$ symmetry. $\mathrm{Cu}$ is shown in yellow, O (blue), $\mathrm{Cl}$ (green), and $\mathrm{Ca}$ (red). The Na dopants substitute at the Ca site. (b) Schematic of the pressure cell for $\mathrm{Ca}_{2-x} \mathrm{Na}_{x} \mathrm{CuO}_{2} \mathrm{Cl}_{2}$, with representative crystals with flat, welldefined cleaved surfaces. $\mathrm{Ca}_{2-x} \mathrm{Na}_{x} \mathrm{CuO}_{2} \mathrm{Cl}_{2}$ cleaves between the ionic $\mathrm{CaCl}$ rocksalt layers. From [114].

described in [115]. One of the peculiarities of the cuprate oxyhalide families (including $\mathrm{Sr}_{2} \mathrm{CuO}_{2} \mathrm{Cl}_{2}$ and $\mathrm{Ca}_{2} \mathrm{CuO}_{2} \mathrm{Cl}_{2}$ ) is that these compounds are extremely hydroscopic and will decompose and turn a bright blue color upon exposure to water or moisture. As a result, all sample preparation and handling was performed in a dry nitrogen environment before insertion in the UHV chamber for our measurements. Furthermore, the oxyhalides are perhaps the most stoichiometric of all the cuprate families, making them ideal for studies of the undoped Mott insulator. Unlike other materials, such as $\mathrm{La}_{2-x} \mathrm{Sr}_{x} \mathrm{CuO}_{4+\delta}, \mathrm{Bi}_{2} \mathrm{Sr}_{2} \mathrm{CaCu}_{2} \mathrm{O}_{8+\delta}, \mathrm{YBa}_{2} \mathrm{Cu}_{3} \mathrm{O}_{7-\delta}, \mathrm{Bi}_{2} \mathrm{Sr}_{2} \mathrm{CuO}_{6+\delta}$, and $\mathrm{Nd}_{2-x} \mathrm{Ce}_{x} \mathrm{CuO}_{4+\delta}$, the oxygen stoichiometry is fixed at 2 oxygen atoms per formula unit, and additional oxygen atoms cannot be introduced by intercalation or annealing, so that the stoichiometry is set entirely by the dopant cation. In addition, $\mathrm{Ca}_{2} \mathrm{CuO}_{2} \mathrm{Cl}_{2}$ and $\mathrm{Sr}_{2} \mathrm{CuO}_{2} \mathrm{Cl}_{2}$ are believed to have very low amount of cation disorder or cross-substitution, unlike materials such as $\mathrm{Bi}_{2} \mathrm{Sr}_{2} \mathrm{CaCu}_{2} \mathrm{O}_{8+\delta}$ [108]. In 

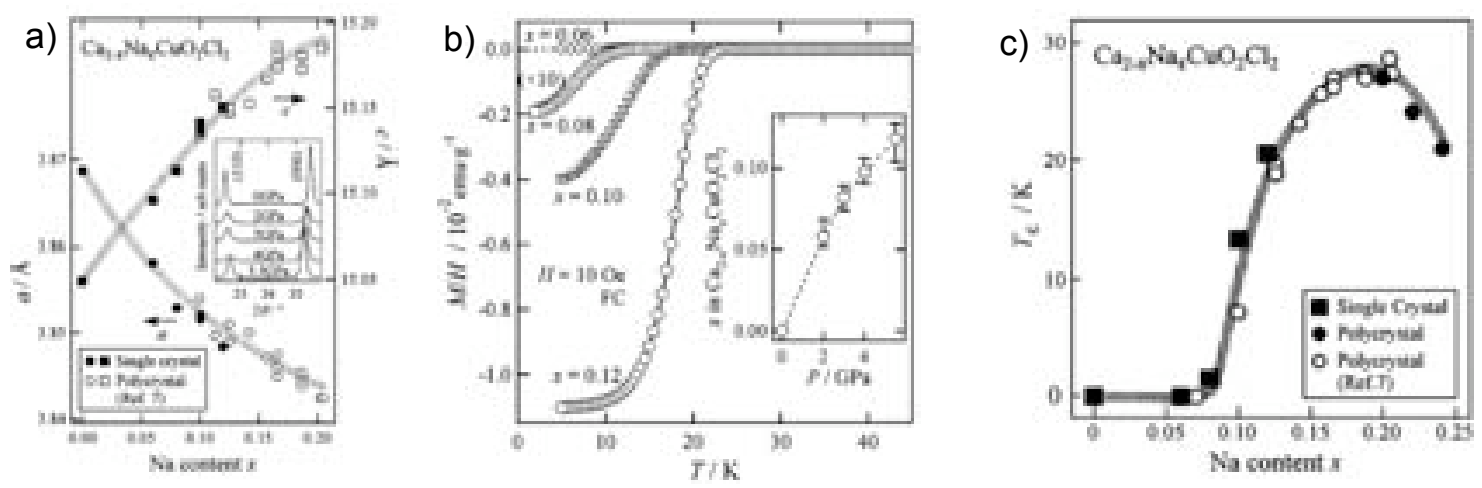

Figure 3.2: (a) Dependence of lattice constants on Na doping. (b) SQUID magnetization measurements of $\mathrm{Ca}_{2-x} \mathrm{Na}_{x} \mathrm{CuO}_{2} \mathrm{Cl}_{2}$. (c) Superconducting phase diagram of $\mathrm{Ca}_{2-x} \mathrm{Na}_{x} \mathrm{CuO}_{2} \mathrm{Cl}_{2}$ from (a) and (b). From [114].

fact, substituting $\mathrm{Na}$ for $\mathrm{Ca}$ is so energetically unfavorable that growth of the doped $\mathrm{Ca}_{2-x} \mathrm{Na}_{x} \mathrm{CuO}_{2} \mathrm{Cl}_{2}$ compounds can only be performed under extremely high pressures ( $\sim 4 \mathrm{GPa})$. Superconductivity in polycrystalline $\mathrm{Ca}_{2-x} \mathrm{Na}_{x} \mathrm{CuO}_{2} \mathrm{Cl}_{2}$ was first discovered by Hiroi, Kobayashi, and Takano in 1994 [116]. However, not until 2000 were the first mm-sized single crystals of $\mathrm{Ca}_{2-x} \mathrm{Na}_{x} \mathrm{CuO}_{2} \mathrm{Cl}_{2}$ grown by Kohsaka et al. [114] using a cubic diamond-anvil high-pressure apparatus with a sample space of $\sim 1 \mathrm{~cm}^{3}$. Details of the high pressure growth can be found in [114].

To characterize the $\mathrm{Na}$ concentration of $\mathrm{Ca}_{2-x} \mathrm{Na}_{x} \mathrm{CuO}_{2} \mathrm{Cl}_{2}$, changes in the lattice parameter as a function of doping were studied, and shown in Figure 3.2a, taken from [114]. The in-plane lattice constant, $a$, shrunk as a function of doping by about $0.03 \%$ per $0.01 \mathrm{Na}$, and $\mathrm{Na}$ concentrations were determined from electron probe microanalysis. Superconducting transition temperatures were measured using SQUID magnetometry, as shown in Figure 3.2b. Knowing this information, one can then plot out the superconducting phase diagram, shown in Figure 3.2c. In addition to the $\mathrm{T}_{\mathrm{c}}$ and the structure, other measurements have been performed on $\mathrm{Ca}_{2-x} \mathrm{Na}_{x} \mathrm{CuO}_{2} \mathrm{Cl}_{2}$, including resistivity, optical, muon spin resonance, and STM measurements. Because of the small and thin nature of the crystals, measurements requiring large bulk crystals, such as neutron scattering, have not yet been performed. STM measurements will be discussed in more detail in Chapter 7 . 

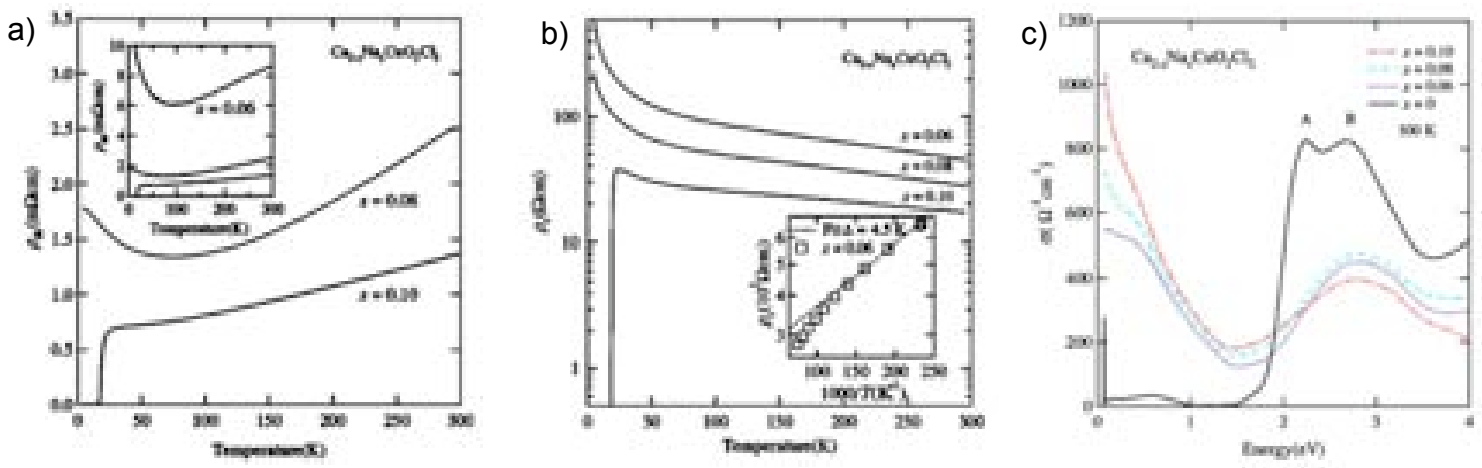

Figure 3.3: (a) In-plane resistivity $\rho_{a b}$ of $x=0.06,0.08$, and 0.10. (b) Out-of-plane resistivity $\rho_{c}$. (c) Optical conductivity spectra of $\mathrm{Ca}_{2-x} \mathrm{Na}_{x} \mathrm{CuO}_{2} \mathrm{Cl}_{2}$ from KramersKronig transformation of the measurements of the reflectivity. From [117].

Resistivity measurements of $\rho_{a b}$ and $\rho_{c}$ are shown in Figure 3.3 taken from [117], and were generally difficult to perform because of the large $\rho_{c} / \rho_{a b}$ anisotropy, the irregular shape of the crystals, and the presence of small flux bubbles in the samples. For instance, the unusually large values of $\rho_{a b}$ for $x=0.06$ (in comparison with $x=0.08$ and 0.10 ) in Figure 3.3a is likely due some small admixture of the much larger $\rho_{c}$ component. However, the transport properties show the characteristic hallmarks of the underdoped cuprates, with "metallic" behavior at high temperatures with an insulating upturn for lightly doped samples at temperatures below $\sim 100$ K. Nevertheless, this ostensibly metallic behavior is still in apparent violation of the Mott-Ioffe-Regel limit [118], which is the semi-classical expectation that the resistivity should saturate at a value corresponding to a mean free path $\sim d$, the lattice spacing. In the cuprates, this corresponds to slightly less than $1 \mathrm{~m} \Omega \mathrm{cm}$. We note that even though this insulating upturn is not discernible in the $x=0.10$ sample, a small insulating upturn is often seen even in heavily underdoped yet still superconducting samples, as in $\mathrm{La}_{2-x} \mathrm{Sr}_{x} \mathrm{CuO}_{4}$ [36]. The values for $\rho_{c} / \rho_{a b}$ were typically on the order of $10^{4}$. Optical spectroscopy measurements were also similar to other cuprates lightly doped cuprates [37], with a large charge-transfer gap of approximately $2 \mathrm{eV}$ in the undoped material, and the emergence of a Drude peak with doping as well as a filling in of spectral weight into the mid-infrared, as shown in Figure 3.3b. In the 


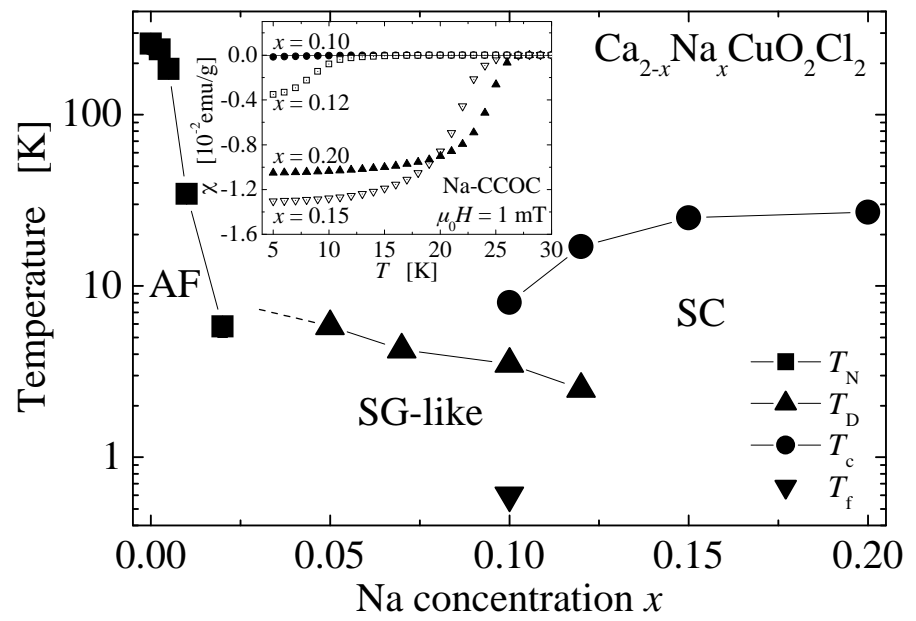

Figure 3.4: Magnetic phase diagram of $\mathrm{Ca}_{2}{ }_{-x} \mathrm{Na}_{x} \mathrm{CuO}_{2} \mathrm{Cl}_{2}$ derived from zero-field $\mu$ SR. From Ohishi et al. [119].

absence of neutron scattering results, the magnetic phase diagram of polycrystalline $\mathrm{Ca}_{2-x} \mathrm{Na}_{x} \mathrm{CuO}_{2} \mathrm{Cl}_{2}$ was plotted out using zero-field $\mu \mathrm{SR}$ by Ohishi et al. [119]. A summary of these results is shown in Figure 3.4, where the Néel order is found to persist to approximately $x=0.02$, followed by an intervening spin glass phase for $0.02<x<0.12$, and an onset of bulk SC for $x>0.10$. This general picture is very similar to the situation observed in other materials, such as $\mathrm{La}_{2-x} \mathrm{Sr}_{x} \mathrm{CuO}_{4}$ [28].

\subsection{Preparation and Characterization of $\mathrm{Ca}_{2-x} \mathrm{Na}_{x} \mathrm{CuO}_{2} \mathrm{Cl}_{2}$ Samples}

Here we detail the preparation and characterization of $\mathrm{Ca}_{2-x} \mathrm{Na}_{x} \mathrm{CuO}_{2} \mathrm{Cl}_{2}$ samples for our ARPES experiments. As mentioned previously, due to the extremely hydroscopic nature of the $\mathrm{Ca}_{2-x} \mathrm{Na}_{x} \mathrm{CuO}_{2} \mathrm{Cl}_{2}$ crystals, all preparations of crystals were performed in a dry nitrogen environment, including ex situ Laue alignment of the crystals, before samples were transported under vacuum to the load lock of the ARPES endstation. Because all ARPES measurements were performed under UHV conditions, and the 

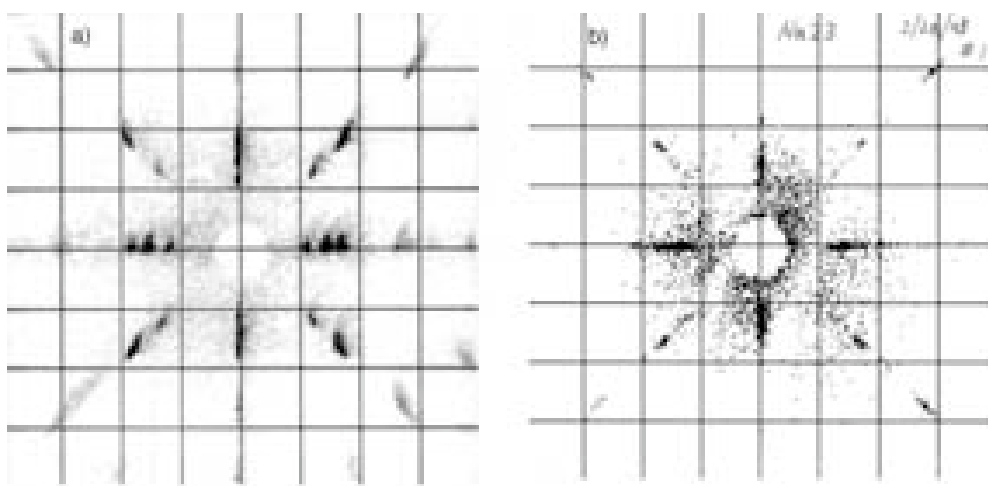

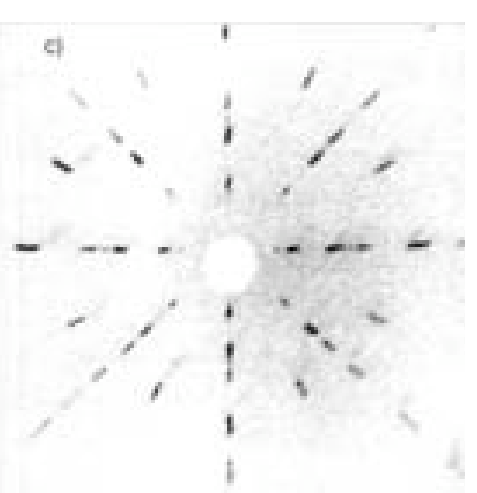
Figure 3.5: Laue diffraction measurements of (a) $\mathrm{Sr}_{2} \mathrm{CuO}_{2} \mathrm{Cl}_{2}$, (b) Figure 3.5: Laue diffraction measurements of (a) $\mathrm{Sr}_{2} \mathrm{CuO}_{2} \mathrm{Cl}_{2}$, (b) $\mathrm{Ca}_{1.88} \mathrm{Na}_{0.12} \mathrm{CuO}_{2} \mathrm{Cl}_{2}$, and (c) $\mathrm{Sr}_{2} \mathrm{RuO}_{4}$. All samples were aligned so that the $a$ and $b$ axes are horizontal and vertical.

samples were cleaved in situ thereby removing any reacted surface layers, bulk degradation of the samples was not a major issue. However, the hydroscopic nature of the samples may have meant that the initially pristine surface layer was potentially more reactive and thus prone to surface aging from adsorbates. We found that the typical sample lifetime of $\mathrm{Ca}_{2-x} \mathrm{Na}_{x} \mathrm{CuO}_{2} \mathrm{Cl}_{2}$ crystals ranged from 12-24 hours, depending on vacuum conditions, making its surface slightly more reactive than typical $\mathrm{Bi}_{2} \mathrm{Sr}_{2} \mathrm{CaCu}_{2} \mathrm{O}_{8+\delta}$ samples, but no more reactive in our vacuum chamber than compounds such as $\mathrm{MgB}_{2}$ or $\mathrm{La}_{2-x} \mathrm{Sr}_{1+x} \mathrm{Mn}_{2} \mathrm{O}_{7}$. However, due to the small supply of doped $\mathrm{Ca}_{2-x} \mathrm{Na}_{x} \mathrm{CuO}_{2} \mathrm{Cl}_{2}$ crystals, we were not able to perform many challenging "sample-hungry" (i.e. a high failure rate) experiments, such as cycling the doped samples through a wide temperature range, as we were able to do for the undoped $\mathrm{Ca}_{2} \mathrm{CuO}_{2} \mathrm{Cl}_{2}$ crystals where a large batch of crystals was available.

Samples were first aligned by Laue diffraction ex situ using a digital Laue diffractometer. A Laue diffraction pattern for undoped $\mathrm{Sr}_{2} \mathrm{CuO}_{2} \mathrm{Cl}_{2}$ is shown in Figure 3.5a. The sample is aligned so that the $\mathrm{Cu}-\mathrm{O}$ bond direction ( $a$ and $b$ axes) run horizontal and vertical, and diffraction lines are also observed along the (110) diagonal direction. In Figure 3.5b, we show a diffraction pattern from $\mathrm{Ca}_{1.88} \mathrm{Na}_{0.12} \mathrm{CuO}_{2} \mathrm{Cl}_{2}$, which is qualitatively very similar to the pattern from $\mathrm{Sr}_{2} \mathrm{CuO}_{2} \mathrm{Cl}_{2}$. In Figure 3.5c, we show a diffraction pattern from $\mathrm{Sr}_{2} \mathrm{RuO}_{4}$ which shares the same $\mathrm{K}_{2} \mathrm{NiF}_{4}$ structure 


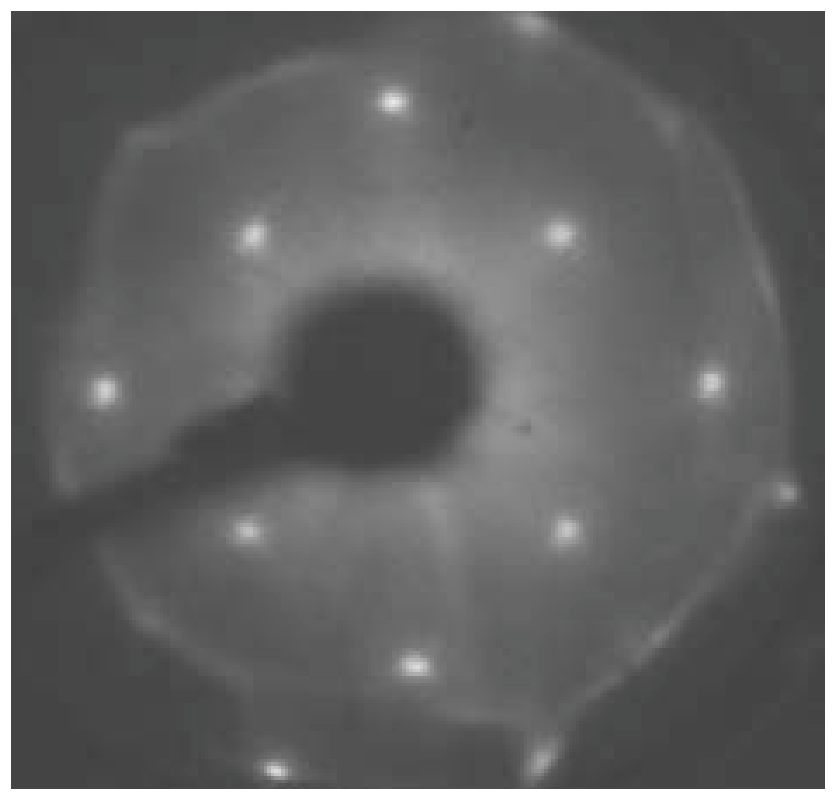

Figure 3.6: Low-energy electron diffraction (LEED) measurements of $\mathrm{Ca}_{1.9} \mathrm{Na}_{0.10} \mathrm{CuO}_{2} \mathrm{Cl}_{2}$ taken at $15 \mathrm{~K}$, showing the clear tetragonal symmetry of the surface structure. LEED pattern is oriented such that the $a$ and $b$ axes run along the $45^{\circ}$ diagonals in this picture, and data were taken at $220 \mathrm{~V}$.

as $\mathrm{Sr}_{2} \mathrm{CuO}_{2} \mathrm{Cl}_{2}$ and $\mathrm{Ca}_{2-x} \mathrm{Na}_{x} \mathrm{CuO}_{2} \mathrm{Cl}_{2}$. However, generally $\mathrm{Sr}_{2} \mathrm{RuO}_{4}$ crystals are of much higher quality than the micacious oxyhalides, and exhibit a much better crystal mosaic, as can be seen in Figure 3.5c.

In addition, we have performed low-energy electron diffraction measurements of $\mathrm{Ca}_{2-x} \mathrm{Na}_{x} \mathrm{CuO}_{2} \mathrm{Cl}_{2}$ on samples cleaved at low temperatures in situ. Because this technique uses low-energy electrons, its surface sensitivity is comparable to ARPES and provides a sensitive probe of the surface structure. In fact, one can view the outgoing photoelectron in the photoemission process as a time-reversed LEED state. Our measurements do not appear to show any clear evidence for surface reconstruction, which would be evident as additional diffraction spots. For instance, another transition metal oxide with the same $\mathrm{K}_{2} \mathrm{NiF}_{4}$ structure, $\mathrm{Sr}_{2} \mathrm{RuO}_{4}$, exhibits extra diffraction spots in LEED which arises from a $\sqrt{2} \times \sqrt{2}$ reconstruction of the topmost $\mathrm{RuO}_{6}$ octahedra $[120,121,122]$. The lack of such additional LEED spots would suggest that no strong surface reconstruction exists, although the LEED used for these measurements 
was not adequate for performing detailed investigations or quantitative LEED studies (such as those using the Barbieri/Van Hove Symmetrized Automated LEED package [123]). Therefore at the level of sophistication of the current LEED measurements that we performed, we cannot conclusively rule out the possibility of a very small surface reconstruction. However, in combination with the STM topographs observed by Hanaguri et al., we would likely conclude that any surface structural reconstruction is highly implausible in $\mathrm{Ca}_{2-x} \mathrm{Na}_{x} \mathrm{CuO}_{2} \mathrm{Cl}_{2}$. 


\section{Chapter 4}

\section{Spectral Analysis of the Mott Insulating Cuprates and the Franck-Condon Model}

At a fundamental level, understanding the behavior of the single hole in the antiferromagnetic Mott insulator is a key to understanding the behavior of the doped Mott insulator. While there still exists some controversy as to whether the physics of the idealized doped Mott insulator alone can explain the remarkably high transition temperatures of the superconducting cuprates, there is no doubt that Mott insulators remain one of the most critical problems in field of strongly correlated systems, and perhaps the whole of condensed matter physics. As a result, it will be worthwhile to revisit and reinvestigate the issue of photoemission from the antiferromagnetic Mott insulator. ${ }^{1}$

Although a large body of photoemission work had been performed on the insulating cuprates for the better part of a decade, there existed a number of rather critical problems regarding the interpretation of the data. In this thesis, we will address these outstanding issues and develop a self-consistent approach to resolving these problems, by using a model based on Franck-Condon broadening.

\footnotetext{
${ }^{1} \mathrm{~A}$ portion of the work discussed in this chapter has been published in K.M. Shen et al., Physical Review Letters 93, 267002 (2004).
} 


\subsection{General Electronic Structure}

The parent compounds of the cuprates (and in fact, all cuprates) are derived from materials with sequences of two-dimensional square $\mathrm{CuO}_{2}$ planes and separated by blocking layers. Materials are often classified by the number of $\mathrm{CuO}_{2}$ layers per unit cell. For instance, $\mathrm{La}_{2-x} \mathrm{Sr}_{x} \mathrm{CuO}_{4}$ or $\mathrm{Ca}_{2-x} \mathrm{Na}_{x} \mathrm{CuO}_{2} \mathrm{Cl}_{2}$ would be referred to as "single-layer" compounds, while $\mathrm{Bi}_{2} \mathrm{Sr}_{2} \mathrm{CaCu}_{2} \mathrm{O}_{8+\delta}$ would be referred to as a "doublelayer" or "bilayer" material. Studies of the electronic structure can be significantly complicated by the interactions between individual $\mathrm{CuO}_{2}$ sheets within a bilayer, resulting in bonding and antibonding combinations of the $\mathrm{CuO}_{2}$-derived bands. To avoid this bilayer band splitting, we have chosen to focus on single-layer compounds such as $\mathrm{Ca}_{2-x} \mathrm{Na}_{x} \mathrm{CuO}_{2} \mathrm{Cl}_{2}$.

In $\mathrm{Ca}_{2-x} \mathrm{Na}_{x} \mathrm{CuO}_{2} \mathrm{Cl}_{2}$, the low-lying states $\left(<10 \mathrm{eV}\right.$ from $\left.\mathrm{E}_{\mathrm{F}}\right)$ are comprised of a combination of primarily $\mathrm{Cu} 3 d, \mathrm{O} 2 p$, and $\mathrm{Cl} 3 p$ orbitals. The Ca atom is generally considered to effectively be in a $\mathrm{Ca}^{2+}$ closed shell configuration, and therefore largely chemically inert. On the other hand, the $\mathrm{Cl}$ ion is in the apical site of the octahedron surrounding the $\mathrm{Cu}$ site, and some small overlap between the $\mathrm{Cl} 3 p$ and $\mathrm{Cu}$ orbitals may be expected. We show band structure predictions based on a linear augmented plane-wave (LAPW) calculation using muffin-tin potentials from L.F. Mattheiss [124] in Figure 4.1.

Typical for the cuprates, the relevant low-energy band from the point of view of band structure is a single, half-filled band $\mathrm{Cu} 3 d_{x^{2}-y^{2}}$ - O $2 p_{x, y}$ antibonding band with an approximately $3 \mathrm{eV}$ bandwidth. This can be seen from a simple analysis of the orbitals of the $\mathrm{Cu}$ and $\mathrm{O}$ ions, as illustrated in Figure 4.2. In a cubic environment, the $\mathrm{Cu} 3 d$ orbitals are no longer degenerate, but are split into $t_{2 g}$ and $e_{g}$ orbitals, with the $d_{x y}, d_{y z}$, and $d_{x z}$ orbitals comprising the deeper $t_{2 g}$ levels, while the $d_{x^{2}-y^{2}}$ and $d_{3 z^{2}-r^{2}}$ comprise the $e_{g}$ orbitals. However, the cuprates are tetragonal and the apical bond is elongated. This lower symmetry further lifts the degeneracy between the orbitals, and the lowest energy orbital is now the $3 d_{x^{2}-y^{2}}$ orbital. In the $\mathrm{Cu}^{2+}$ state, this results in a single hole on the $3 d_{x^{2}-y^{2}}$ orbital. The antiferromagnetism in the undoped cuprates then arises from this single uncompensated spin on the $3 d_{x^{2}-y^{2}}$ orbital. On the other 

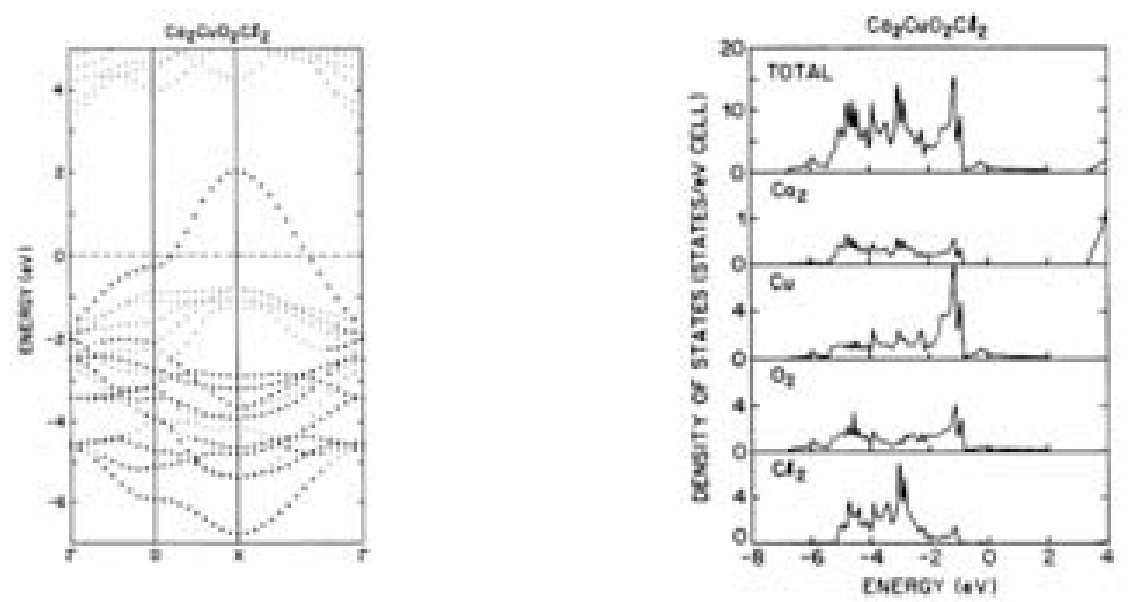

Figure 4.1: Band structure calculations by L.F. Mattheiss [124] for $\mathrm{Ca}_{2} \mathrm{CuO}_{2} \mathrm{Cl}_{2}$. Calculated partial density of states (DOS) from [124].

hand, the $\mathrm{O}^{2-}$ anion has its $2 p^{6}$ orbitals split into $\sigma, \pi_{\|}$, and $\pi_{\perp}$ orbitals by the crystal field. The hybridization between the $\mathrm{Cu}$ and $\mathrm{O}$ then leads to the single antibonding sigma band $\left(\sigma^{*}\right)$ comprised of $\mathrm{Cu} 3 d_{x^{2}-y^{2}}$ and $\mathrm{O} 2 p$ orbitals. However, the undoped cuprates are not half-filled metals, but are, in fact, antiferromagnetic insulators with $\mathrm{a} \sim 2 \mathrm{eV}$ gap. This insulating behavior arises from the Coulomb repulsion between electrons; in particular, it is the energy difference between moving an electron from the $\mathrm{Cu} 3 d$ to an empty orbital on the $\mathrm{O} 2 p$, as discussed in Chapter 1.

A more detailed and rather sophisticated band structure calculation of the oxychloride materials was produced by Hayn et al. [125]. These calculations utilize a combination of local density approximation + Coulomb repulsions (LDA $+\mathrm{U}$ ) and experimental ARPES data to extract effective tight-binding parameters. Although these specific calculations are for the $\mathrm{Sr}_{2} \mathrm{CuO}_{2} \mathrm{Cl}_{2}$ compound, and not $\mathrm{Ca}_{2} \mathrm{CuO}_{2} \mathrm{Cl}_{2}$, we will still discuss these calculations in the context of $\mathrm{Ca}_{2} \mathrm{CuO}_{2} \mathrm{Cl}_{2}$. We believe this justified as both the $\mathrm{Sr}$ and $\mathrm{Ca}$ ions are essentially fully ionized and thus are electronically quite inert. Therefore, the only effective difference between $\mathrm{Sr}_{2} \mathrm{CuO}_{2} \mathrm{Cl}_{2}$ and $\mathrm{Ca}_{2} \mathrm{CuO}_{2} \mathrm{Cl}_{2}$ is the difference in ionic radius of the cation and thus different lattice constants. In addition, the ARPES data of $\mathrm{Sr}_{2} \mathrm{CuO}_{2} \mathrm{Cl}_{2}$ and $\mathrm{Ca}_{2} \mathrm{CuO}_{2} \mathrm{Cl}_{2}$ generally appear extremely similar. 


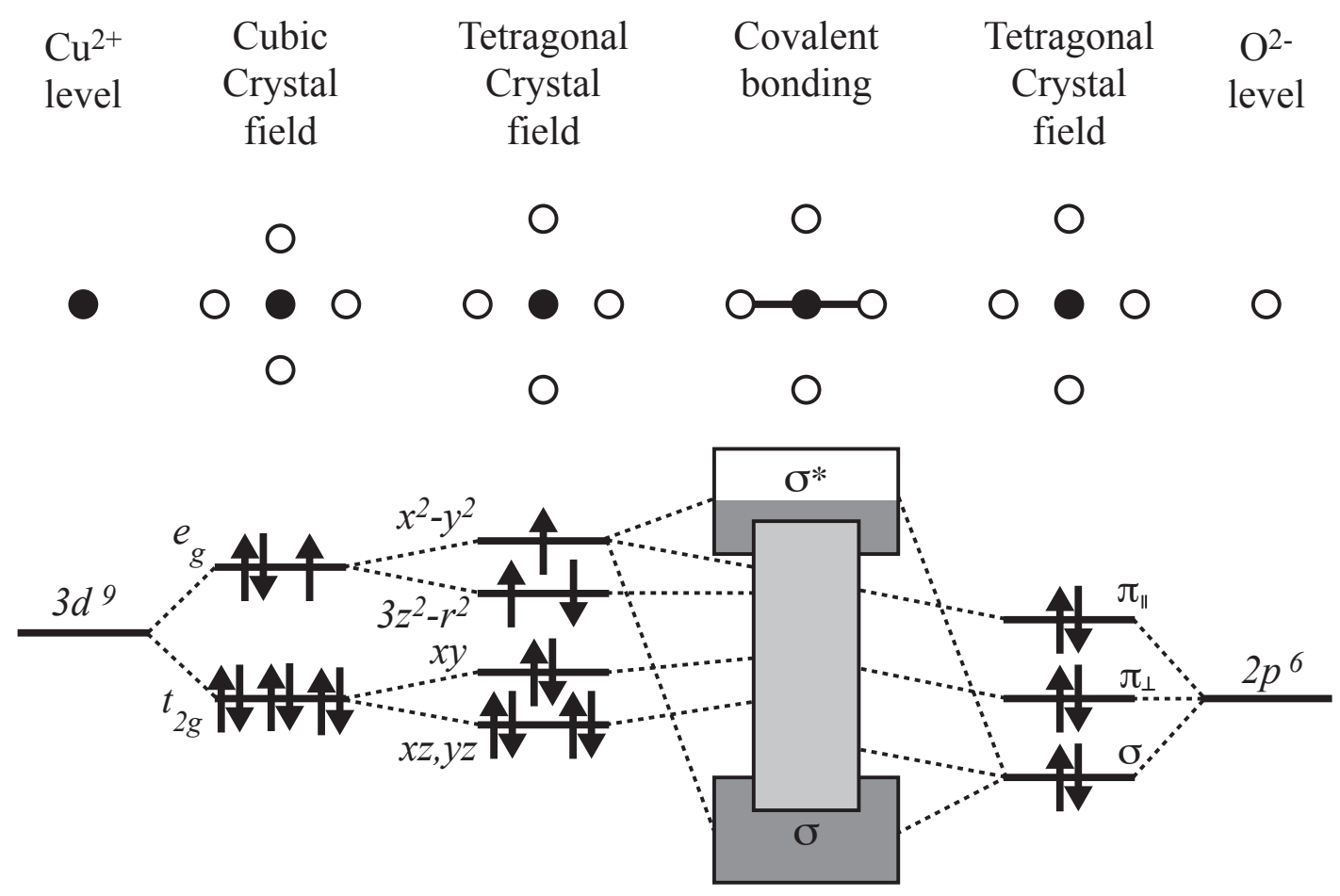

Figure 4.2: Schematic showing the effects of cubic and tetragonal crystal field splitting on the orbital levels of $\mathrm{Cu}^{2+}$ and $\mathrm{O}^{2-}$, and the effects of covalent bonding.

\subsubsection{ARPES Studies of the Valence Band}

Using the calculations in [125] as a reference, we identify three particular electronic features in the valence band which will later study in detail and which we show in Figure 4.3. The first is the famed Zhang-Rice singlet (ZRS) [24] feature (Figure 4.3b), which is the lowest-lying excitation and the electronic feature of most interest, and will be discussed in greater detail later. The second feature, at $\sim 2.5 \mathrm{eV}$ binding energy shown in Figure $4.3 \mathrm{c}$, is the $\mathrm{O} 2 p_{\pi}$ orbital. It was previous identified as the so-called " $1 \mathrm{eV}$ peak" in a number of different cuprates, such as $\mathrm{YBa}_{2} \mathrm{Cu}_{3} \mathrm{O}_{7-\delta}$ and $\mathrm{Sr}_{2} \mathrm{CuO}_{2} \mathrm{Cl}_{2}$, and thus an intrinsic feature of the $\mathrm{CuO}_{2}$ plane. Work by Pothuizen [126] identified this state as having $\mathrm{O} 2 p_{\pi}$ symmetry by comparison to a tight-binding model of the $\mathrm{CuO}_{2}$ plane; this was also later confirmed in [125]. The work by Pothuizen et al. used an eight-band tight-binding model ( $5 \mathrm{Cu} 3 d$ orbitals and $3 \mathrm{O} 2 p$ orbitals) 
to explain the wavefunction character of the $\mathrm{O} 2 p_{\pi}$ state. The work by Hayn et al. utilized a more extensive LDA and LDA+U calculations with 11 individual bands, but agreed qualitatively with the assignment of the $\mathrm{O} 2 p_{\pi}$ state by Pothuizen. Our identification of the $\mathrm{O} 2 p_{z}$ state was made by combining the information from the $\mathrm{Sr}_{2} \mathrm{CuO}_{2} \mathrm{Cl}_{2}$ valence band with the LDA and $\mathrm{LDA}+\mathrm{U}$ calculations. One important aspect of this state is that at $\mathbf{k}=(\pi, \pi)$, it is in an in-plane antibonding configuration, and has zero overlap (i.e. completely nonbonding) with the Zhang-Rice singlet or $\mathrm{Cu}$ $3 d_{x^{2}-y^{2}}$ orbital, as shown in Figure 4.3c. We will later take advantage of this fact in our data analysis of the chemical potential shift and the assignment of lattice polaron formation in undoped $\mathrm{Ca}_{2} \mathrm{CuO}_{2} \mathrm{Cl}_{2}$. The third state that we will identify is the $\mathrm{O}$ $2 p_{z}$ state at $\sim 4 \mathrm{eV}$ binding energy (Figure $4.3 \mathrm{a}$ ), although there may some small admixture of the $\mathrm{Cl} 3 p_{z}$ orbital, as well.

Band structure calculations and ARPES data on both $\mathrm{Ca}_{2} \mathrm{CuO}_{2} \mathrm{Cl}_{2}$ and $\mathrm{Sr}_{2} \mathrm{CuO}_{2} \mathrm{Cl}_{2}$ indicate that at $\mathbf{k}=(0,0)$, the $\mathrm{O} 2 p_{z}$ is relatively well isolated from the other electronic states which will help in identifying its peak position. Like the $\mathrm{O} 2 p_{\pi}$ state, the $\mathrm{O} 2 p_{z}$ band is also completely non-bonding with the Zhang-Rice singlet or the $\mathrm{Cu} 3 d_{x^{2}-y^{2}}$ orbitals, but at $\mathbf{k}=(0,0)$. Within the range of -7 to $-4 \mathrm{eV}$ in binding energy, there is a large manifold of $\mathrm{Cu} 3 d$ and $\mathrm{O} 2 p$ states. Because these peaks are strongly overlapping and the assignment of these orbitals depends sensitively on the band structure calculations, it is difficult to reliably determine the nature of each of these states, although this was attempted in Ref. [125].

In fact, we have found that the valence band of $\mathrm{Ca}_{2} \mathrm{CuO}_{2} \mathrm{Cl}_{2}$ can vary considerably from sample to sample, even when measured under ostensibly identical conditions. In particular, we have identified two characteristic types of valence bands, which we denote as A and B, as shown in Figure 4.4. These spectra were recorded using $h \nu=25.5$ $\mathrm{eV}$ at the $(0,0)$ point under essentially identical conditions. Most importantly, we found that the LHB and low-energy features were basically indistinguishable between samples of type A and B, and therefore did not affect the essential low-energy physics which we were attempting to address. One possibility is that these two different lineshapes could have originated from flux inclusions which were present on the cleaved surfaces. Supporting this assumption, we found that all samples grown by Y. Kohsaka 

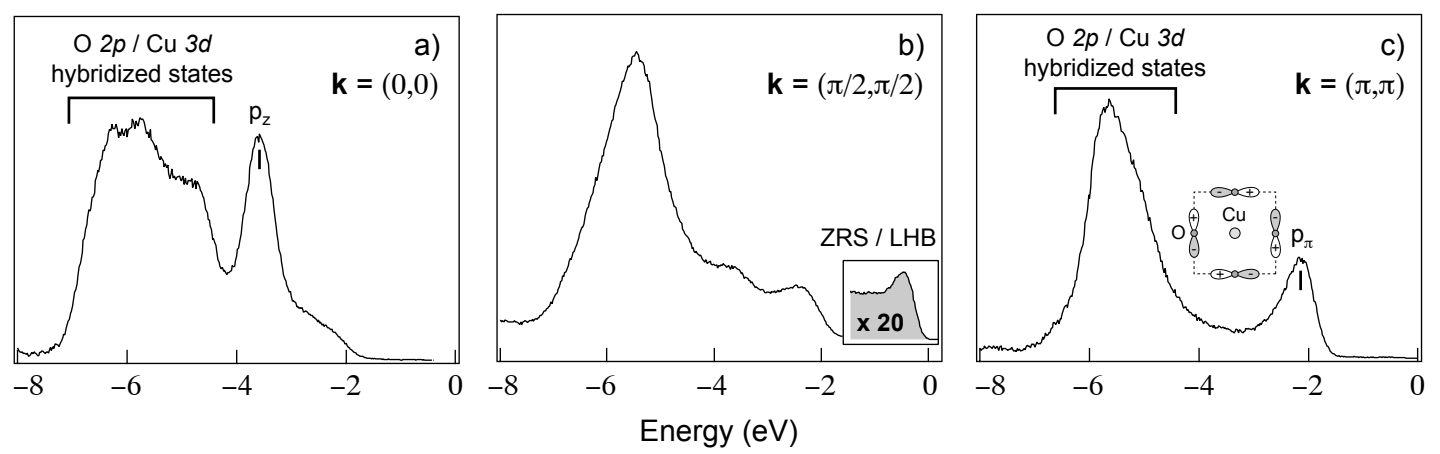

Figure 4.3: Valence band spectra of $\mathrm{Ca}_{2} \mathrm{CuO}_{2} \mathrm{Cl}_{2}$ taken using $h \nu=25.5 \mathrm{eV}$ after the subtraction of an inelastic Shirley background. Spectra are shown at $(0,0)$ (a); $(\pi / 2, \pi / 2)(\mathrm{b})$, with the ZRS peak shown on an expanded scale; and $(\pi, \pi)(\mathrm{c})$.

using the high-pressure flux method (over 30 doped and undoped cleaves) exhibited valence bands of type A, while the undoped samples grown by L.L. Miller using an ambient pressure flux technique exhibited valence bands which varied between lineshapes of type A and B. Since these crystals should be identical in all other ways (and the LHB and $\mathrm{O} 2 p_{\pi}$ features behave identically across these two batches), this would indicate that the flux inclusions introduced by these two growth methods should be different. Because of the uniformity of the lineshapes observed in samples grown by the high-pressure method, one could assume that $\mathrm{A}$ is representative of a pure, cleaved $\mathrm{Ca}_{2} \mathrm{CuO}_{2} \mathrm{Cl}_{2}$ or $\mathrm{Ca}_{2-x} \mathrm{Na}_{x} \mathrm{CuO}_{2} \mathrm{Cl}_{2}$ surface, and that $\mathrm{B}$ represents the additional contribution of flux inclusions. On the other hand, if B was due to polycrystalline flux inclusions, one would expect extra, non-dispersive peaks in the valence band. However, while the spectra at $(0,0)$ in samples of types $\mathrm{A}$ and $\mathrm{B}$ are very different, the spectra at $(\pi, \pi)$ are very similar, making the possibility of polycrystalline inclusions highly unlikely. Moreover, the peaks in B are roughly at the same position of the peaks in A, but primarily with different intensities, so that no extra peaks are clearly evident. This raises the possibility that the samples of type $\mathrm{B}$ may be $\mathrm{Sr}_{2} \mathrm{CuO}_{2} \mathrm{Cl}_{2}$ single crystals that were accidentally sent and included in the batch of $\mathrm{Ca}_{2} \mathrm{CuO}_{2} \mathrm{Cl}_{2}$ crystals. This would be consistent with the fact that no high-pressure $\mathrm{Ca}_{2} \mathrm{CuO}_{2} \mathrm{Cl}_{2}$ crystals exhibited type B valence bands, that the low energy peaks in B showed very similar dispersions to $\mathrm{A}$, and the valence band spectra from type B samples appear 

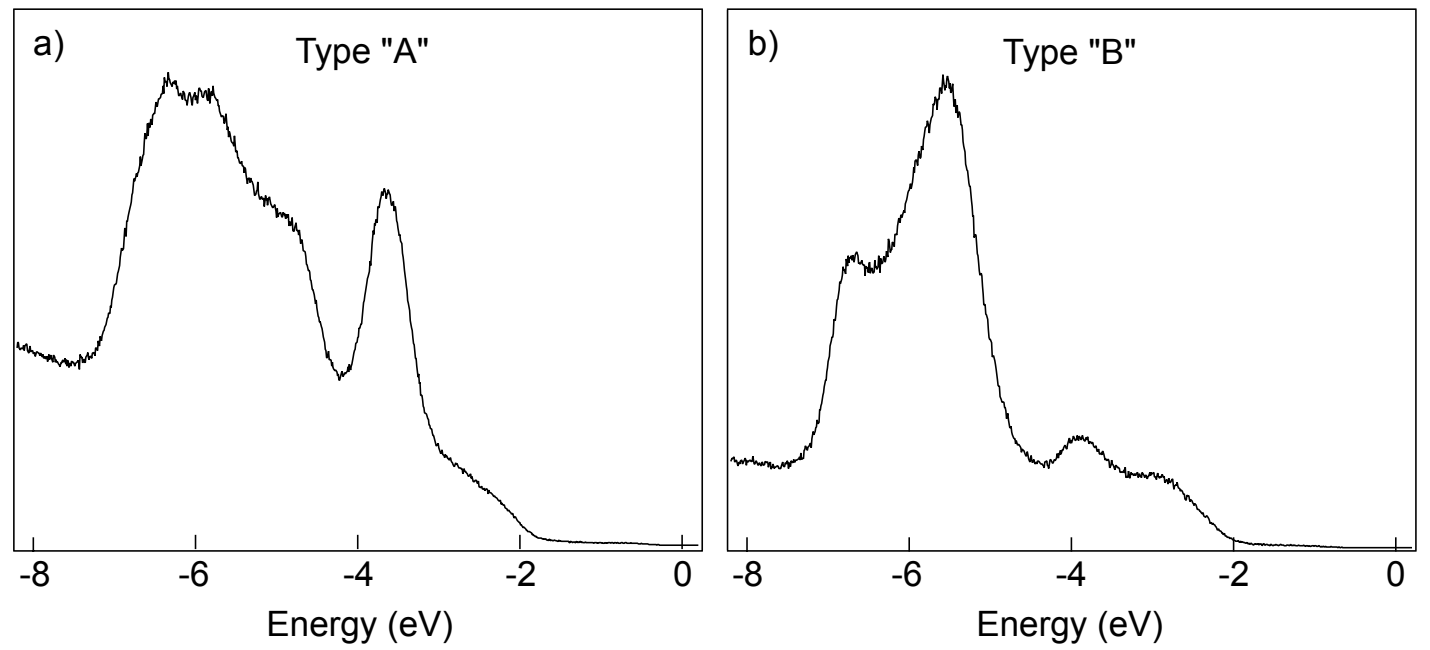

Figure 4.4: Raw valence band spectra of $\mathrm{Ca}_{2} \mathrm{CuO}_{2} \mathrm{Cl}_{2}$ taken at $(0,0)$ using $h \nu=25.5$ $\mathrm{eV}$, under identical conditions. Spectra in (a) of Type A were taken on undoped $\mathrm{Ca}_{2} \mathrm{CuO}_{2} \mathrm{Cl}_{2}$ grown using the high-pressure technique, while spectra of Type $\mathrm{B}$ were taken on $\mathrm{Ca}_{2} \mathrm{CuO}_{2} \mathrm{Cl}_{2}$ grown under ambient pressure. The difference between $\mathrm{A}$ and $\mathrm{B}$ are presumably either due to flux inclusions or accidentally mislabeled $\mathrm{Sr}_{2} \mathrm{CuO}_{2} \mathrm{Cl}_{2}$ samples.

rather similar to data from known $\mathrm{Sr}_{2} \mathrm{CuO}_{2} \mathrm{Cl}_{2}$ samples.

Additional valence peaks, presumably also from flux inclusions of $\mathrm{NaCl}$ or $\mathrm{NaClO}_{4}$, were also observed by Filip Ronning in the doped $\mathrm{Ca}_{2-x} \mathrm{Na}_{x} \mathrm{CuO}_{2} \mathrm{Cl}_{2}$ samples grown under high pressures. However, these peaks appeared at higher energies $(\sim-10 \mathrm{eV})$, while the low-energy valence band still resembled lineshape A. The work in [127] demonstrated that these peaks disappeared over the course of a few hours. Our later work confirmed that these peaks were being photodesorbed as a function of photon flux, and not being suppressed from residual gases in the vacuum chamber. However, this type of flux inclusion is likely different than any possible inclusions in B samples, since no photo-induced changes were observed in samples of type B.

Another possibility that we should address is that these two different signals could arise from different surface terminations (either the $\mathrm{CaCl}$ or $\mathrm{CuO}_{2}$ layers). However, we believe this is highly improbable for a number of reasons. The weak bonding and mirror symmetry between the ionic $\mathrm{CaCl}$ double layers make this the obvious choice 
for a cleavage plane, and this is borne out by XPS [128]. Secondly, these materials are very electronically two-dimensional $\left(\rho_{c} / \rho_{a b} \sim 10^{4}\right)$ and can be cleaved using very little force, suggesting easy and preferential cleavage between the ionic $\mathrm{CaCl}$ layers, and not between the $\mathrm{CaCl}$ and $\mathrm{CuO}_{2}$ layers. Finally, and most importantly, this would simply not explain the dependence of the valence band lineshape on the growth method.

\subsubsection{ARPES Studies of the Lower Hubbard Band}

At the most fundamental level, it is important to understand how the ARPES data relate to the expectations for the single-electron excitations from the band structure calculations and the $t-J$ model, respectively. Such a comparison of these different scenarios will be essential in determining which model comes closest to approximating the "real world". However, extracting an experimental "band dispersion" from ARPES is a highly nontrivial matter, as there are many possible metrics for determining dispersion. Usually, the simplest way to address this problem is to use an approach based simply on the non-interacting band picture. In this case, a sharp pole in $\mathcal{A}(\mathbf{k}, \omega)$ will appear where $\omega=\varepsilon(\mathbf{k})$. In a weakly interacting Fermi-liquid scenario, interactions will remove spectral weight from the pole $(Z<1)$, shift the pole position to $\omega=\varepsilon(\mathbf{k})+\Sigma^{\prime}(\omega, \mathbf{k})$, and distribute the rest of the weight $(1-Z)$ to other energies, but this sharp peak structure will be retained. Much of this work deals with the failings of such a naïve model, especially when dealing with very strongly correlated systems when $Z \ll 1$. However, we will start by using this weakly interacting model to interpret the data, as this has been the basis for the analysis of ARPES data for decades.

ARPES data on the insulating cuprate $\mathrm{Ca}_{2} \mathrm{CuO}_{2} \mathrm{Cl}_{2}$ are shown in Figure 4.5 at low energies, with data taken along different high-symmetry lines of the Brillouin zone, which are qualitatively similar to the first work by Wells [50]. The most apparent feature in the data is a broad but still well-defined dispersive peak within $\sim 1 \mathrm{eV}$ of $\mathrm{E}_{\mathrm{F}}$. Typically, the most strongly dispersive features are observed along the $(0,0)-(\pi, \pi)$ line, or along the AF zone boundary. Only weak dispersion is observed along the $(0,0)-(\pi, 0)$ line, while the situation is more complex for the 

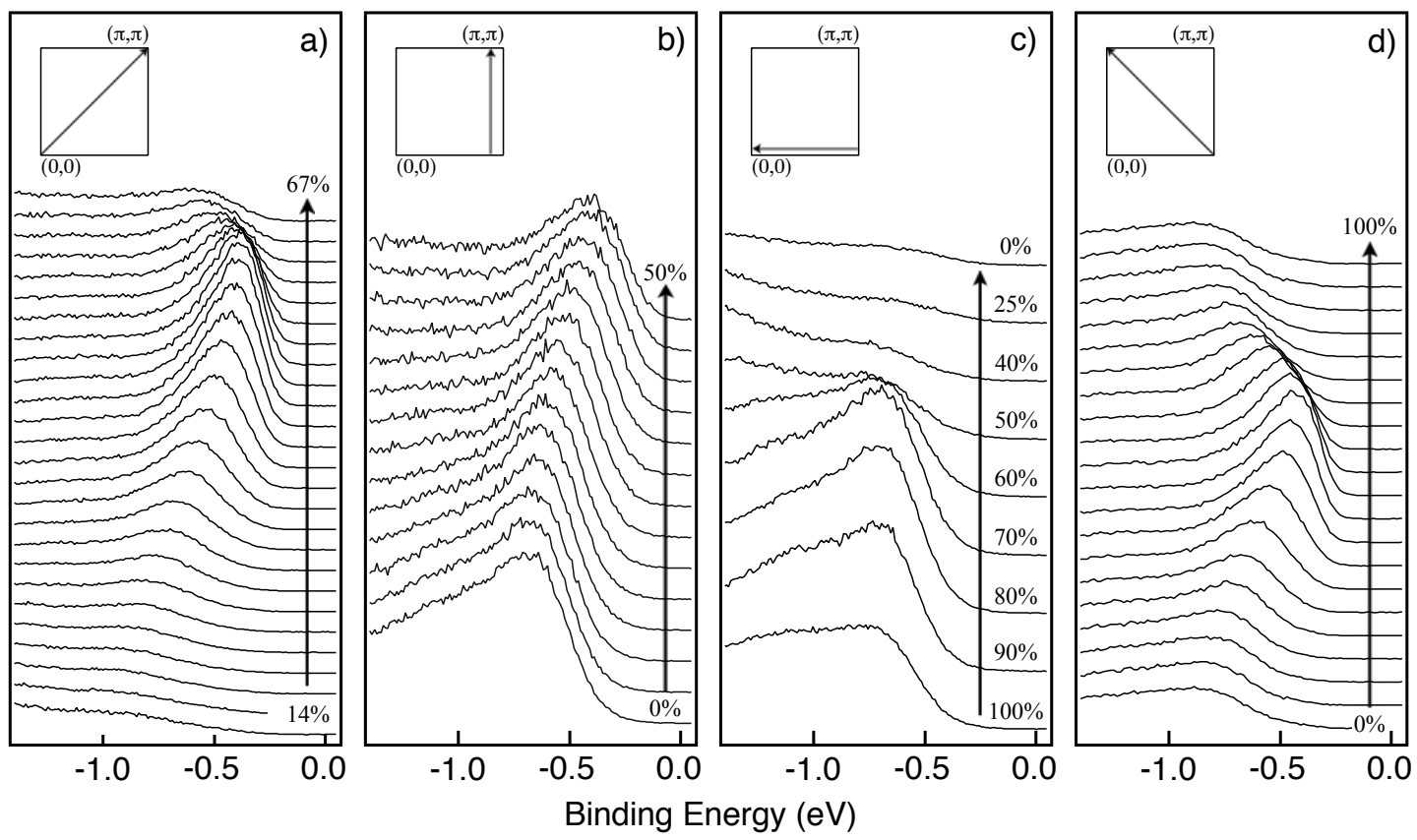

Figure 4.5: Panels (a)-(d) show energy distribution curves (EDCs) taken from $\mathrm{Ca}_{2} \mathrm{CuO}_{2} \mathrm{Cl}_{2}$ along various high symmetry lines of the crystal. Data were taken at $200 \mathrm{~K}$ with $h \nu=25.5 \mathrm{eV}$. From bottom to top: (a) EDCs along $(0,0)-(\pi, \pi)$; (b) $(0.85 \pi, 0)-(0.85 \pi, \pi)$; (c) $(\pi, 0)-(0,0)$; and $(\mathrm{d})(\pi, 0)-(0, \pi)$. EDCs in panel $(\mathrm{d})$ were symmetrized across $(\pi / 2, \pi / 2)$.

$(\pi, 0)-(\pi, \pi)$ direction. At $(\pi, 0)$ in $\mathrm{Ca}_{2} \mathrm{CuO}_{2} \mathrm{Cl}_{2}$, the photoemission matrix element for the LHB always appears rather weak, and the states are rather indistinct. Therefore, it has been difficult to clearly determine any obvious dispersion along $(\pi, 0)$ $(\pi, \pi)$, regardless of the photon energy used. However, very clear dispersion is observed along a parallel cut very close to the $(\pi, 0)-(\pi, \pi)$ line (for instance, along $(0.85 \pi, 0)-(0.85 \pi, \pi))$. Because there should be rather little change in the dispersion in going from $(0.85 \pi, 0)-(0.85 \pi, \pi)$ to $(\pi, 0)-(\pi, \pi)$, we will use the obtained dispersion from $(0.85 \pi, 0)-(0.85 \pi, \pi)$, but use larger error bars to reflect the uncertainty in going from $(0.85 \pi, 0)$ to $(\pi, 0)$.

By using the standard weak-coupling analysis and empirically tracking the local peak maximum in the EDCs as a function of momentum, we can attempt to extract an experimental dispersion, $E(\mathbf{k})$. This was done as early as 1995 in the pioneering 

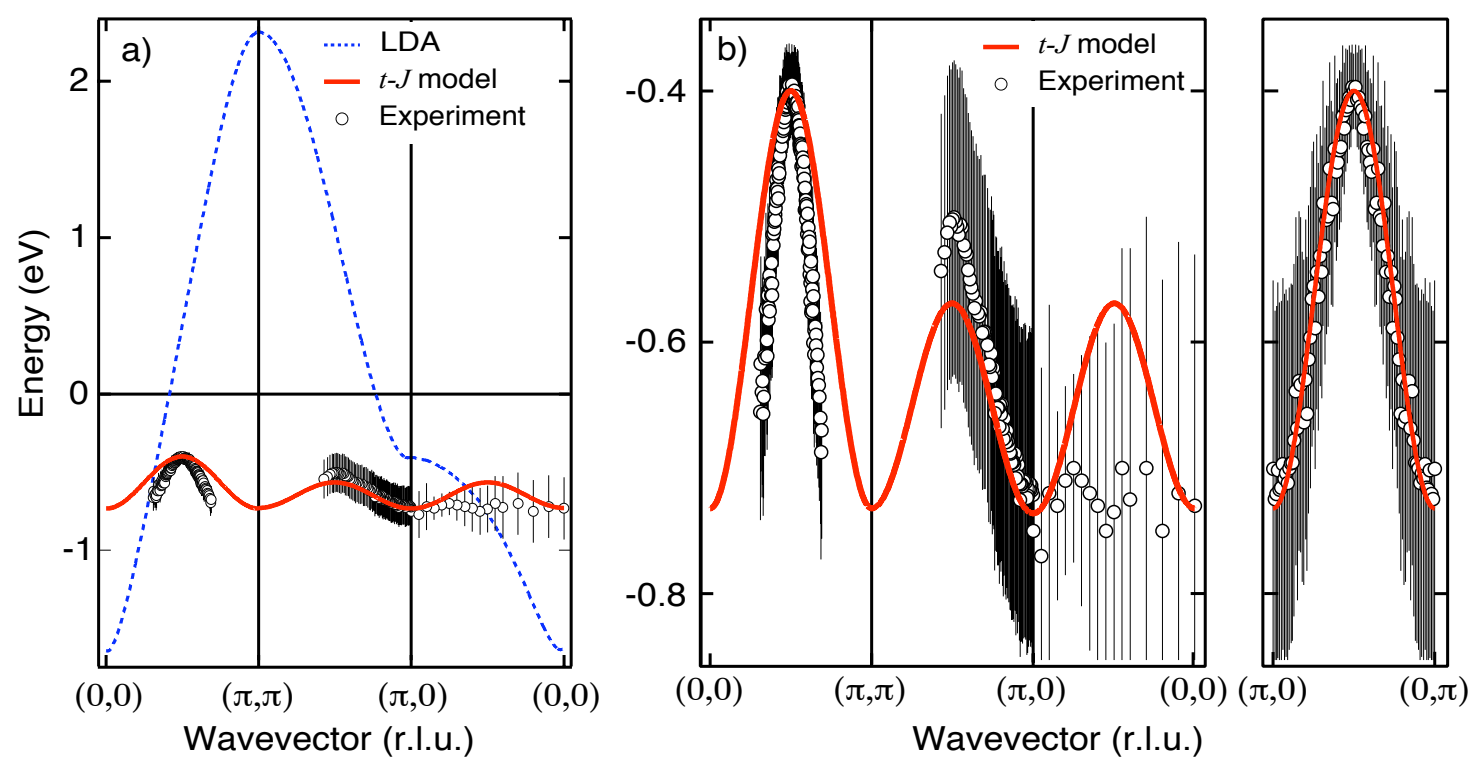

Figure 4.6: (a) Dispersion of LHB peak maximum in $\mathrm{Ca}_{2} \mathrm{CuO}_{2} \mathrm{Cl}_{2}$ along high symmetry lines (circles), along with predictions from LDA band structure [125] and fits to extended $t-J$ model calculations [129]. Error bars represent uncertainty in locating peak position, not the peak width. (b) Close-up of experimentally derived dispersion and comparison with fits to $t-J$ model calculations, including also data along $(\pi, 0)-(0, \pi)$.

work of Barry Wells et al., and repeated many times since. With the use of many more samples and high data sampling rates available with the Scienta 200 series analyzers, we can greatly improve on the data quality found in earlier work. This is shown in Figure 4.6, where we plot the peak position as a function of momentum, $E(\mathbf{k})$. Despite the advances in instrumentation and sample growth, the qualitative features compared to the work from [50] are basically unchanged.

Figure 4.6 shows the failure of the one-electron band structure in describing the data. The dashed lines show the LDA bandstructure taken from Hayn et al. (although this is for $\mathrm{Sr}_{2} \mathrm{CuO}_{2} \mathrm{Cl}_{2}$, the band structure is extremely close to the results from Mattheiss for $\mathrm{Ca}_{2} \mathrm{CuO}_{2} \mathrm{Cl}_{2}$ ). The solid black line shows results from [129], which is a fitting of the self-consistent Born approximation calculation of the $t-t^{\prime}-t^{\prime \prime}-J$ model to the function form shown in Equation 4.1 
$E(k)=-0.55 J\left(\cos \left(k_{x}\right)+\cos \left(k_{y}\right)\right)^{2}-4 t_{e f f}^{\prime} \cos \left(k_{x}\right) \cos \left(k_{y}\right)-2 t_{e f f}^{\prime \prime}\left(\cos \left(2 k_{x}\right)+\cos \left(2 k_{y}\right)\right)$

with $J=0.14 \mathrm{eV}, t_{\text {eff }}^{\prime}=-0.038 \mathrm{eV}$, and $t_{\text {eff }}^{\prime \prime}=0.022 \mathrm{eV}$. These values of $t_{\text {eff }}^{\prime}$ and $t_{e f f}^{\prime \prime}$ are simply a useful parameterization of the dispersion, and not so physically relevant. The failure of the LDA calculations is unsurprising, as all experimental probes clearly indicate that $\mathrm{Ca}_{2} \mathrm{CuO}_{2} \mathrm{Cl}_{2}$ is not a half-filled metal, but rather an antiferromagnetic insulator. What is more remarkable is the level of agreement between the $\mathrm{Ca}_{2} \mathrm{CuO}_{2} \mathrm{Cl}_{2}$ data and calculations using the $t-J$ model. These can basically be summarized as follows:

- Bandwidth Renormalization: While the one-electron calculations predict a bandwidth of $8 t \sim 3-4 \mathrm{eV}$, the $t-J$ model predicts a bandwidth of $2 J \sim 0.3 \mathrm{eV}$. This is in rough agreement with our experimental value of $\sim 0.3-0.4 \mathrm{eV}$.

- Symmetry of Dispersion: The dispersion of the LHB is found experimentally to be symmetric about the AF zone boundary, at least along the $(0,0)-(\pi, \pi)$ line (about $\mathbf{k}=(\pi / 2, \pi / 2))$. This is in accordance with a Néel-ordered antiferromagnet, consistent with the $t-J$ model, but obviously not with the band picture.

- Overall Dispersion: The overall dispersion agrees roughly with the expectations based on the $t-J$ model, although the main disagreement is along the AF zone boundary $(0, \pi)-(\pi, 0)$. Adding higher-order hopping terms $\left(t^{\prime}, t^{\prime \prime}\right)$ can help to improve the fit of this extended $t-t^{\prime}-t^{\prime \prime}-J$ model to the experimental data, although the question of the physical relevance of this extended model is still up for debate.

- Quasiparticle Weight: The $t-J$ models predict a $Z \sim 0.2$, due to the effects of magnetic interactions. Compared to other states in the valence band, the LHB peaks have much weaker intensity, consistent with this reduced $Z$. 
Therefore, it is clear from this simple analysis of the insulating $\mathrm{Ca}_{2} \mathrm{CuO}_{2} \mathrm{Cl}_{2}$ data that calculations based on the $t-J$ model do a far better job of approximating the ARPES spectra than simple band structure calculations. However, in the Figure 4.6b, one can observe a fairly clear discrepancy between the calculations and the experimental data. One possibility is that the value used for $J$ in the calculations is smaller than reality. Another is the existence of a more dispersive, high energy branch near $(0,0)$ which skews our peak position to higher binding energies, particularly near $(\pi / 4, \pi / 4)$, where the LHB intensity becomes very weak, as shown in Figure 5.12. Therefore, the points near $(\pi / 4, \pi / 4)$ in Figure $4.6 \mathrm{~b}$ may not be and accurate representation of the dispersion of the antiferromagnetic Zhang-Rice singlet band alone. Still, for quite some time, this approximate agreement was essentially taken as a demonstration of the success of the $t-J$ model in describing the single-hole dynamics in the AF Mott insulator. Despite this apparent success of the $t-J$ model, there were still a number of discrepancies between theory and experiment, which were either difficult to address or not fully understood or realized. The next section will discuss these discrepancies in some detail, and then later I shall describe a model based on Franck-Condon broadening (FCB) which can resolve these problems.

\subsubsection{Failure of the Coherent Quasiparticle Scenario}

From aforementioned analysis, the agreement of the $t-J$ model and experiment was predicated on the use of the standard "weakly-interacting" paradigm where the ARPES peak represented the QP pole in the spectral function. Moreover, the $t-J$ model predicts a $Z \sim 0.2$ in the lowest-energy QP peak. While this is substantially reduced from 1 (due to spin effects), this implies that the eigenstates of the $N-1$ system of the $t-J$ model still have a reasonably strong overlap $(\sim 20 \%)$ with the pure electron/hole states. Despite the strong interactions, numerical calculations of the $t-J$ model indeed still predict very sharp QP-like excitations. Although on a superficial level this type of analysis appears to give satisfactory results, there are crucial flaws and inconsistencies related to such a scheme. The obvious discrepancy between the experiment and theory was the strongly broadened peak widths observed 
in the insulator. This was first pointed out by the work of Pothuizen et al. [126], and later by Changyoung Kim [130], who suggested a heuristic multiple initial state / final state model as a source of the broadening and temperature dependent change in spectral weight. The newer work presented here borrows on these earlier ideas, and builds on it by using a particular model (FCB) and physical scenario to describe the single-electron excitations.

At this point, we will describe the failings of the weakly-interacting quasiparticle scenario, which we dub the "Coherent Quasiparticle Scenario", or CQS. More accurately, CQS refers to the scenario when the projection of the $N-1$ state (i.e. the initial state, with one electron instantaneously removed, $c_{\mathrm{k}} \psi_{i}^{N}=\psi_{i}^{N-1}$ ) onto the true eigenstates of $N-1$ system is rather large. In other words, this would be the case when $\psi_{i}^{N}$ has some finite overlap with a noninteracting many-body wavefunction, such as would be the case for a Fermi liquid. However, when there are strong interactions (electron-electron, or electron-phonon, for instance), this coupling of the single electronic states (to other electrons, or to the lattice) will effectively reduce the overlap between the eigenstates of the $N-1$ system (which are then heavily dressed) and $\psi_{i}^{N-1}$, thereby "stealing" spectral weight away from the quasiparticle peak. This would then be the limit where $Z \ll 1$, which we will discuss in detail. In particular, we will concentrate on one particular model where $Z \ll 1$, namely the situation for Franck-Condon broadening (FCB).

We will first discuss the failings of the CQS in the context of the ARPES data on $\mathrm{Ca}_{2} \mathrm{CuO}_{2} \mathrm{Cl}_{2}$, and later, how the $\mathrm{FCB}$ can resolve these issues. This will prove to be particularly important in Chapter 6, when we discuss the evolution in the electronic structure as a function of hole doping, where the CQS will again prove inadequate, and the FCB model proves to be a much more attractive alternative. We outline a list of four problems with the CQS in the context of $\mathrm{Ca}_{2} \mathrm{CuO}_{2} \mathrm{Cl}_{2}$ :

1. Peak Width: This is the longest-standing and best-known problem regarding ARPES data on the undoped parent Mott insulators. When ARPES data were first taken on $\mathrm{Sr}_{2} \mathrm{CuO}_{2} \mathrm{Cl}_{2}$ ten years ago, the experimental resolution was still rather poor, so it was unclear whether this peak width was, in fact, intrinsic. As the instrumental resolution improved, it became clear that the peaks 

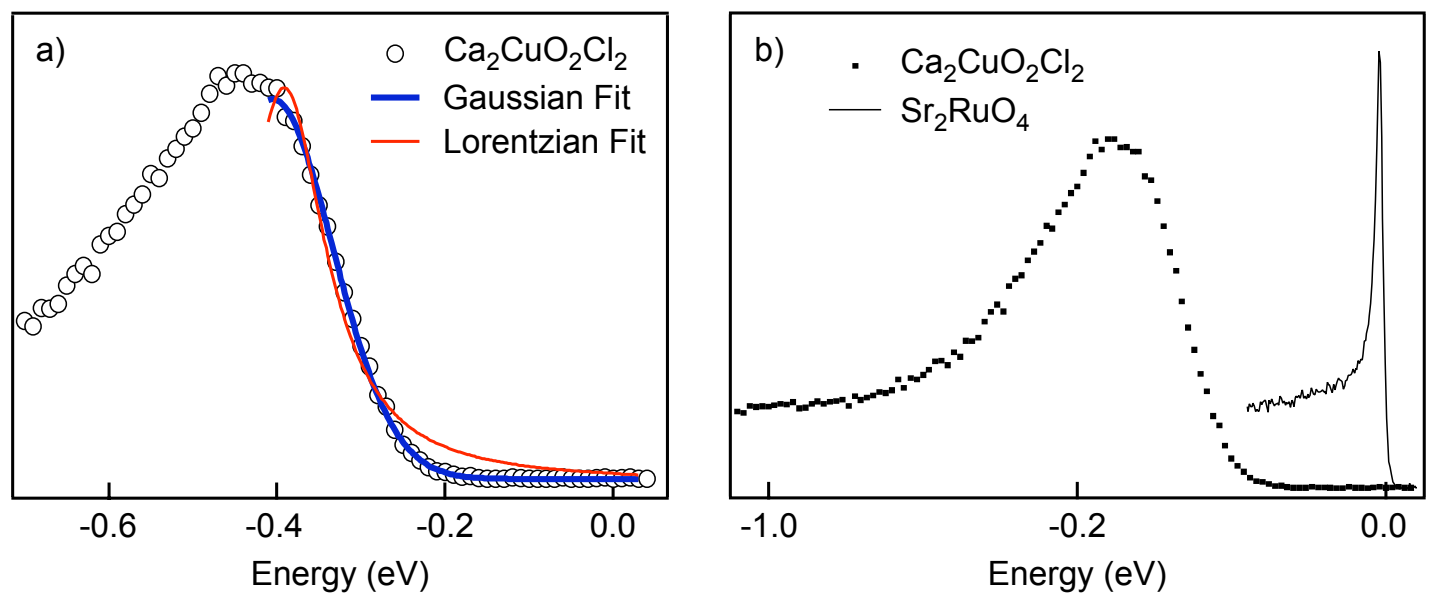

Figure 4.7: (a) Fits to the experimental data from $\mathrm{Ca}_{2} \mathrm{CuO}_{2} \mathrm{Cl}_{2}$ at the top of the valence band, $\mathbf{k}=(\pi / 2, \pi / 2)$, at $200 \mathrm{~K}$. Fits to a Lorentzian are shown in red, while the Gaussian is shown in blue. (b) Comparison of the data from $\mathrm{Ca}_{2} \mathrm{CuO}_{2} \mathrm{Cl}_{2}$ in (a) with $\mathrm{Sr}_{2} \mathrm{RuO}_{4}$, taken at $15 \mathrm{~K}$ at $\mathbf{k}=(\pi, 0)$ (peak represents the surface $\gamma$-band).

measured in $\mathrm{Sr}_{2} \mathrm{CuO}_{2} \mathrm{Cl}_{2}$ and $\mathrm{Ca}_{2} \mathrm{CuO}_{2} \mathrm{Cl}_{2}$ were much broader than other compounds. This can be seen in Figure 4.7b, where data at the top of the valence band of $\mathrm{Ca}_{2} \mathrm{CuO}_{2} \mathrm{Cl}_{2}$ are compared to truly quasiparticle-like excitations measured in $\mathrm{Sr}_{2} \mathrm{RuO}_{4}$. At the top of the valence band, there is no phase space for electron-electron scattering, and Fermi-liquid theory would predict $\delta$-functionlike excitations there (and at all energies less than the gap). Instead, the peaks measured in $\mathrm{Ca}_{2} \mathrm{CuO}_{2} \mathrm{Cl}_{2}$ were found to have a width comparable to the entire bandwidth of the lower Hubbard band - a highly unusual situation.

2. Lineshape: Typical quasiparticle poles should possess a lineshape reminiscent of a Lorentzian. This can be seen from inspection of the form of $\mathcal{A}(\mathbf{k}, \omega)=$ $\frac{\left(\Sigma^{\prime \prime} \mathbf{k}, \omega\right)^{2}}{\left(\omega-\varepsilon_{k}-\Sigma^{\prime}(\mathbf{k}, \omega)\right)^{2}+\Sigma^{\prime \prime}(\mathbf{k}, \omega)^{2}}$. When at the pole energy, $\omega=\varepsilon_{k}+\Sigma^{\prime}(\mathbf{k}, \omega)$, the form is approximately Lorentzian if $\frac{d \Sigma(\mathbf{k}, \omega)}{d \omega}$ is not too large over the range of the pole width, so that $\Sigma^{\prime}(\mathbf{k}, \omega)$ and $\Sigma^{\prime \prime}(\mathbf{k}, \omega)$ can be well approximated by constants, as discussed in Chapter 2. However, what is observed is that the falloff of the spectral weight away from the peak is suppressed exponentially. In fact, the low-energy half of the peak of the LHB can be fit extremely well by using 
a Gaussian form. This can be seen in Figure 4.7a. At higher energies, there should be additional higher energy states [131], likely leading to the asymmetric lineshapes. On the other hand, a Lorentzian fits the ARPES data poorly; the large peak widths would imply very long tails away from the peak, something that is not observed experimentally.

One could argue that an exponential tail in the density of states to $E_{F}$ might be expected from impurity states (as would be in the case for a doped semiconductor, i.e. Urbach tails). However, the undoped system is stoichiometric and very robust against disorder (making $\mathrm{Ca}_{2} \mathrm{CuO}_{2} \mathrm{Cl}_{2}$ the ideal system for studies of the Mott insulator). Because the entire peak can be fit by a Gaussian, this would imply that a very large number of these states arise from impurities, which would simply be far too great a concentration. Secondly, if this was due to impurity states, this exponential tail would be essentially isotropic (a density of states effect). However, as we will show later, the width of this Gaussian is surprisingly momentum dependent. Moreover, this Gaussian lineshape is also present for other deeper states in the valence band $\left(\mathrm{O} 2 p_{\pi}\right)$, which are far removed from $\mathrm{E}_{\mathrm{F}}$. Finally, adding additional impurities in the form of Na dopant ions actually makes the low-energy features sharper, not broader. All of these factors would suggest that this unusual Gaussian lineshape cannot solely be due to the presence of impurity states.

3. Chemical Potential Positions: Another apparent inconsistency plaguing the CQS is the position of the chemical potential, $\mu$, relative to the LHB peak. In a pure insulator, $\mu$ is poorly defined, and can exist anywhere within the gap. However, its upper and lower bounds are set by the bottom of the conduction band and the top of the valence band, respectively. More specifically, the position of the QP pole at the top of the valence band should set the ultimate lower bound for $\mu$. In a photoemission experiment, $\mu$ in the insulator is most likely determined by surface defects and impurities. From measuring many samples, we have observed a distribution of $\mu$ 's relative to the LHB peak, and have 

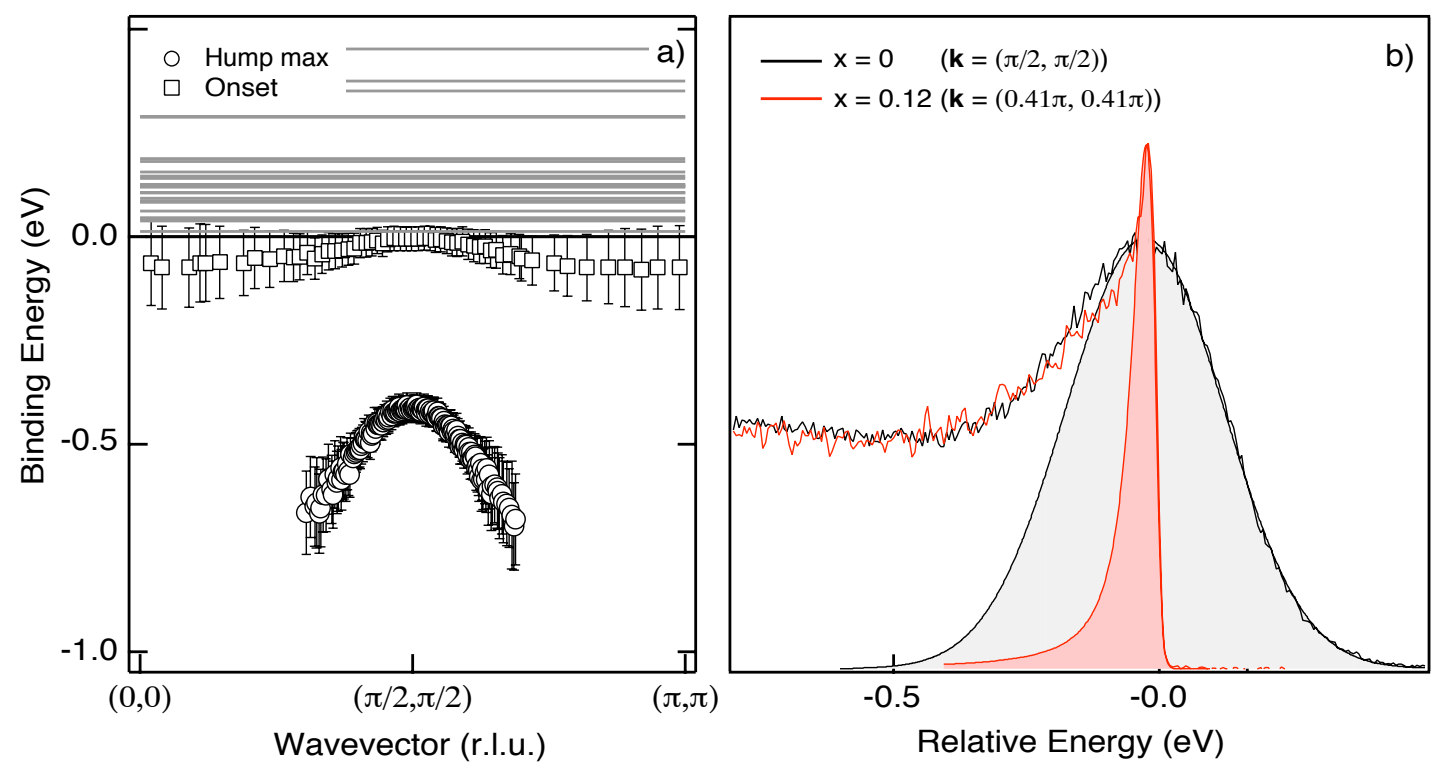

Figure 4.8: (a) Pinned positions of $\mu$ in $\mathrm{Ca}_{2} \mathrm{CuO}_{2} \mathrm{Cl}_{2}$, plotted relative to the peak position of the LHB (open circles), and the onset of spectral weight (open squares), along the $(0,0)-(\pi, \pi)$ direction. Experimentally determined values of $\mu$, relative to the LHB peak, are shown as horizontal grey lines. (b) Lineshape of undoped $\mathrm{Ca}_{2} \mathrm{CuO}_{2} \mathrm{Cl}_{2}$ (black) and $\mathrm{Ca}_{2-x} \mathrm{Na}_{x} \mathrm{CuO}_{2} \mathrm{Cl}_{2}$ (red) at $\mathbf{k}_{\mathrm{F}}$. Data were taken at $h \nu=25.5 \mathrm{eV}$ and normalized to the high binding energy background at $-1 \mathrm{eV}$; normalizing to the valence band gives very similar results. Estimated peak intensities are shown as shaded curves.

determined a lower bound for $\mu$. This is shown in Figure 4.8 where the experimentally determined $\mu$ 's from many samples are plotted as horizontal grey lines. However, the lower bound for $\mu$ is set approximately $400 \mathrm{meV}$ above the position of the LHB peak, not close to the LHB peak position. In fact, $400 \mathrm{meV}$ is a very large energy scale - comparable to the bandwidth of the entire LHB. Therefore, there would appear to be some sort of "hidden energy scale" which is keeping $\mu$ from approaching the LHB peak, something that will become clear when we discuss the FCB model.

4. Evolution of Spectral Weight: As the cuprates become progressive more hole doped, they are generally believed to recover Fermi-liquid-like behavior near the overdoped side of the phase diagram. Therefore, one would expect as cuprates 
become more hole doped that the QP residue, $Z$, should increase monotonically. However, because of the large width of the LHB peak in $\mathrm{Ca}_{2} \mathrm{CuO}_{2} \mathrm{Cl}_{2}$, the integrated spectral weight of this peak is rather sizable. One can compare this to the intensity of the QP-like peaks observed in the doped materials. Because these QP-like peaks are much sharper than the LHB peaks, they actually encompass much less spectral weight than the broad LHB peak, as shown in Figure 4.8b. Data here were taken under similar conditions $\left(\mathrm{Ca}_{2} \mathrm{CuO}_{2} \mathrm{Cl}_{2}\right.$ was measured at $300 \mathrm{~K}$; $\mathrm{Ca}_{1.88} \mathrm{Na}_{0.12} \mathrm{CuO}_{2} \mathrm{Cl}_{2}$ was measured at $15 \mathrm{~K}$, although this should not make a large difference), using $h \nu=25.5 \mathrm{eV}$. The spectra were normalized to the flat background at $-1 \mathrm{eV}$. Normalizing to the intensity of the entire valence band gave qualitatively similar results. To estimate the peak weights, we use the Gaussian fit for $\mathrm{Ca}_{2} \mathrm{CuO}_{2} \mathrm{Cl}_{2}$, and an empirical fit to the peak for $\mathrm{Ca}_{1.88} \mathrm{Na}_{0.12} \mathrm{CuO}_{2} \mathrm{Cl}_{2}$. The ratio of these peak areas, $\mathrm{A}_{0.12} / \mathrm{A}_{0}$, is estimated to be roughly 0.20 . Although the fitting scheme is somewhat arbitrary and there may also be different photoelectron matrix elements for the doped and undoped states, this analysis should be accurate to within a factor of 2 . This would still imply that under the CQS, " $Z_{0}$ " $>Z_{0.12}$, which is a highly nonintuitive and unusual (and incorrect) result; we will demonstrate that this apparent " $Z_{0}$ " is incorrect and arises from inconsistencies in the CQS.

\subsection{Franck-Condon Model}

The previous section has outlined reasons for the failure of the conventional, weakcoupling CQS and has demonstrated the need for a new model to understand the ARPES data on the undoped and lightly doped cuprates. Here, we will outline a model based on Franck-Condon broadening (FCB) to explain many of the unusual spectral features in the cuprates. The most important point of this model is that in this picture, $Z \rightarrow 0$. In principle, a different model which possesses this essential feature might also be used to explain the features in the undoped cuprates. However, we will show that FCB can nicely describe some detailed features in the ARPES spectra, such as the Gaussian lineshapes. Therefore, the FCB scenario appears to 
be the best existing model to describe the experimental data, although this certainly does not preclude other candidates.

We will start with a simply physical picture for Franck-Condon broadening, one that is commonly used in quantum chemistry and molecular physics, and is well worth discussing in some detail. Later, we will describe how one can also arrive at the same picture by using a standard Green's function formalism. In the FCB model, we start with an electron in a harmonic potential well, corresponding to the chemical bonding to adjacent atoms. Although the potential need not be exactly harmonic, this should be a good approximation to first order. This is shown in Figure 4.9a, where the potential energy curves are plotted versus a generalized atomic / lattice coordinate, $q$, with an equilibrium position $q_{0}$. Each excited state, $\psi_{m}^{N}$, corresponds to the electronic state with $N$ electrons (there are $N$ electrons in the initial state) and $m$ vibrational quanta excited. We treat the system in the initial state as $\psi_{0}^{N}$, the lower curve in Figure 4.9a, with no vibrational quanta thermally excited (low temperature limit). The photoemission process then ionizes the molecular system to a final state, $\psi_{m}^{N-1}$, plus a photoelectron at "infinity". We then take the sudden approximation (or Born-Oppenheimer approximation), where the outgoing photoelectron is removed so quickly as not to interact with the ionized molecule as it is leaving the system. This photoionization transition can be treated as direct, where the atomic coordinates do not change from $q_{0}$ during the process. However, the ionized molecule will obviously have a different atomic potential and final equilibrium position, $q_{1}$, due to the altered nature of the ionized chemical bond. Therefore, the final states for the photoionized molecule are shown by the upper curve. The key point is that the initial state, $\psi_{0}^{N}$, can overlap with many different final states, $\psi_{m}^{N-1}$, not simply $\psi_{0}^{N-1}$. This can be shown by simply performing the integral of $\left|\left\langle\psi_{0}^{N} \mid \psi_{m}^{N-1}\right\rangle\right|^{2}$, where the $\psi$ 's are simply different harmonic oscillator wavefunctions. A simple example of FCB is shown in Figure 4.9b, where the photoemission spectrum of $\mathrm{H}_{2} \rightarrow \mathrm{H}_{2}^{+}$is shown (from Turner [132]). Even in this simplest of cases, the $\psi_{0}^{N} \rightarrow \psi_{0}^{N-1}$ transition (which we will call the "0-0 transition" or the "zero-phonon line") has only a $\sim 0.10$ probability, and the overlap to the $N-1$ states with vibrational quanta excited, $\psi_{m>0}^{N-1}$, is about $\sim 0.90$. Simply speaking, this basically implies that a photoemitted electron has a high probability 

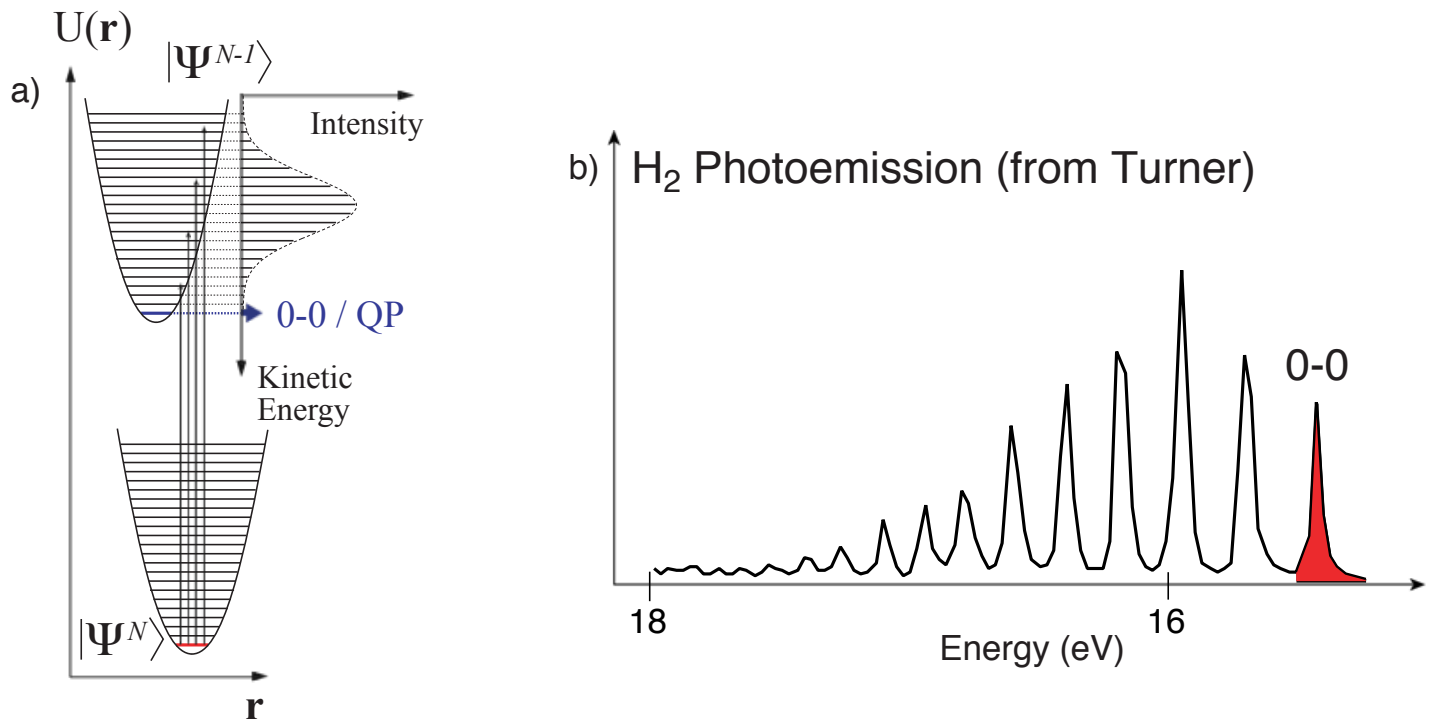

Figure 4.9: (a) Illustration of the Franck Condon principle, showing the transition from the $\psi_{0}^{N} \rightarrow \psi_{m}^{N-1}$ states. (b) Photoemission spectrum from $\mathrm{H}_{2} \rightarrow \mathrm{H}_{2}^{+}$with the 0-0 transition shown in red [132]

of leaving the system in a vibrationally excited state when it leaves the molecule. In fact, the $\psi_{0}^{N} \rightarrow \psi_{1,2,3,4}^{N-1}$ transitions all have a higher probability than $0-0$. This overlap will depend strongly on the nature of the chemical bond (i.e. whether it is a bonding, antibonding, or nonbonding orbital). It is rather easy to see that if the electron somehow does not participate in the chemical bonding (equivalent to a zero coupling constant), that the upper curve should be identical to the lower curve. Then, the 0-0 transition will have a probability of unity, because of the orthogonality of different eigenstates, since the $N$ and $N-1$ systems have the same $q$-Hamiltonian.

Another way to approach this problem is using a Green's function formalism, which extends more naturally to the solid-state limit. In fact, the molecular approach and the Green's function approach describe precisely the same physical scenario and in fact give the same results. Therefore, the only difference between the two approaches is one of mathematical formalism and not physics. However, while the molecular picture may be more physically intuitive, the Green's function approach is more general and powerful. For instance, one can not only address the FCB case, but 
also the weakly interacting scenario (CQS), where the excitations are delocalized, free-electron-like quasiparticle states; the FCB and CQS start from different limits. In Chapter 2, we have already discussed the evolution of the single-particle spectral function with the addition of weak interactions (CQS). For instance, this should quite well reproduce the case of weak electron-electron interactions in a good metal, such as copper. In this case, the real eigenstates of the $N-1$ system have well defined $\mathbf{k}$ quantum numbers and overlap very strongly with the single hole state, $\psi_{i}^{N-1}$. This implies that the many-body eigenstates of the photoexcited $N-1$ system can be nearly constructed from a basis set of delocalized Bloch states, with the interactions causing a slight mixture of these states. This is basically the standard weak-coupling CQS phenomenology that is nearly always utilized to interpret ARPES spectra.

On the other hand, we can take an electronic state which is coupled to some bosonic field. In the solid, this bosonic field could represent spin waves (magnons) or lattice vibrations (phonons). The $\mathcal{H}_{e l-b}$ term in the Hamiltonian will couple the electron (fermionic) and phonon/magnon (bosonic) Hilbert spaces, so that the new eigenstates have mixed electron-boson character, but solving for these new eigenstates becomes far more difficult. Below is a Hamiltonian for a single electronic band with an electronic dispersion, $\epsilon_{\mathbf{k}}$, interacting with a single branch of bosonic excitations through a matrix element, $M_{\mathbf{q}}$.

$$
\mathcal{H}=\sum_{\mathbf{k}, \sigma} \epsilon_{\mathbf{k}} c_{\mathbf{k}, \sigma}^{\dagger} c_{\mathbf{k}, \sigma}+\sum_{\mathbf{q}} \omega_{\mathbf{q}} a_{\mathbf{q}}^{\dagger} a_{\mathbf{q}}+\sum_{\mathbf{k}, \mathbf{q}, \sigma} M_{\mathbf{q}} c_{\mathbf{k}+\mathbf{q}, \sigma}^{\dagger} c_{\mathbf{k}, \sigma}\left(a_{\mathbf{q}}+a_{-\mathbf{q}}^{\dagger}\right)
$$

However, even in the absence of electron-electron interactions, this problem is still analytically intractable. Even without considering electron-electron interactions, a wide array of fascinating phenomena in the solid state such as charge-density-wave (CDW) formation and superconductivity are driven by such electron-boson (in this case, phonon) interactions. However, an exact solution of an extremely simplified case of this interacting fermion-boson model is within our theoretical reach. We will use the solutions of this simplified model to help develop our intuition for interpreting the more complicated situations which exist in real materials. Theoretical attempts 
to obtain either analytical or numerical solutions to the more generalized interacting fermion-boson (IFB) model are well beyond the scope of this thesis. However, we will later discuss some recent theoretical treatments of the interacting electronphonon Hamiltonian which give remarkable agreement with the experimental data and provide some insights and justifications for our work. To simplify the IFB model, we take the case of a localized electronic state coupled to a single bosonic mode. For the purposes of this discussion, we will assume that the boson is a phonon. In this case, $c$ and $c^{\dagger}$ are the electron annihilation (creation) operators, $a_{\mathbf{q}}$ and $a_{\mathbf{q}}^{\dagger}$ are the phonon annihilation (creation) operators, and $M_{\mathbf{q}}$ is the matrix element for the electron-phonon coupling.

$$
\mathcal{H}=\epsilon_{0} c^{\dagger} c+\sum_{\mathbf{q}} \omega_{\mathbf{q}} a_{\mathbf{q}}^{\dagger} a_{\mathbf{q}}+\sum_{\mathbf{q}} M_{\mathbf{q}} c^{\dagger} c\left(a_{\mathbf{q}}+a_{-\mathbf{q}}^{\dagger}\right)
$$

An abbreviated summary of the analytical solution will follow, but the full solution can be found in Mahan [86]. The Green's function for $t>0$ is then

$$
G(t)=-i\left\langle T_{t} c(t) c^{\dagger}\right\rangle=-i e^{-i\left(\epsilon_{0}-\Delta\right)} e^{-\Phi(t)}
$$

where

$$
\Phi(t)=\sum_{\mathbf{q}}\left(\frac{M_{\mathbf{q}}}{\omega_{\mathbf{q}}}\right)^{2}\left[N_{\mathbf{q}}\left(1-e^{i \omega_{\mathbf{q}} t}\right)+\left(N_{\mathbf{q}}+1\right)\left(1-e^{-i \omega_{\mathbf{q}} t}\right)\right]
$$

$\Delta$ is

$$
\Delta=\sum_{\mathbf{q}} \frac{M_{\mathbf{q}}^{2}}{\omega_{\mathbf{q}}}
$$

$N_{\mathbf{q}}$ is the phonon (Bose-Einstein) occupation number

$$
N_{\mathbf{q}}=\frac{1}{e^{\beta \omega_{\mathbf{q}}}-1}
$$

To solve for the spectral function, we neglect any q-dependence in the Hamiltonian. Therefore, we will also assume that this phonon is dispersionless (an Einstein mode, $\left.\omega_{q}=\omega_{0}\right)$, and that the coupling constant between the electrons and phonons 
is momentum-independent (i.e., just a $c$-number, $M$ ). Then Equation 4.3 can be expressed as

$$
\mathcal{H}=\epsilon_{0} c^{\dagger} c+\omega_{0} a^{\dagger} a+M c^{\dagger} c\left(a+a^{\dagger}\right)
$$

The solution to this problem, at $T=0$ (so that $N=0$ ) is then given by

$$
\mathcal{A}(\omega)=e^{-g} \sum_{l=0}^{\infty} \frac{g^{l}}{l !} \delta\left(\omega-\epsilon_{0}+\Delta-l \omega_{0}\right)
$$

It is immediately obvious that $\mathcal{A}(\omega)$ takes on the form of a Poisson distribution of $\delta$-functions separated by $\omega_{0}$. Moreover, this clearly satisfies the sum-rule for $\mathcal{A}(\omega)$ since the Poisson distribution necessarily sums to unity. In this case, the coupling constant $g$ can be expressed as

$$
g=\sum_{\mathbf{q}}\left(\frac{M_{\mathbf{q}}}{\omega_{\mathbf{q}}}\right)^{2} \rightarrow\left(\frac{M}{\omega_{0}}\right)^{2}
$$

The form of $\mathcal{A}(\omega)$ is shown in Figure 4.10. As mentioned earlier, the spectral function takes on the form of a series of $\delta$-functions separated by $\omega_{0}$. Since the chain of $\delta$-functions in Eq. 4.9 are shifted by $\Delta=g \omega_{0}$, this means that the center of gravity of this distribution remains at the unrenormalized electronic energy, $\epsilon_{0}$. The zero-phonon line, corresponding to $l=0$, is shifted to lower binding energies relative to $\epsilon_{0}$. The spectral weight in this zero-phonon line (corresponding to $Z$ in the solid-state) is reduced exponentially quickly as a function of the coupling constant, g. Since the functional form of Eq. 4.9 is a Poisson distribution, this reduces to a simple Gaussian in the limit of large $g$. In fact, even when $g$ is only $\sim 3$, the spectral weight distribution is already very close to a Gaussian form, as can be seen in Figure 4.10. In our later analyses, we will simply use the Gaussian form to substitute for the Poisson distribution.

We can also study the temperature dependence of $\mathcal{A}(\omega)$ by considering how the phonon population, $N$, changes as a function of temperature. At finite temperature, there are thermally excited phonons, and the electron can not only create phonons, but also annihilate phonons. This will broaden the spectral function, as shown in 
Figure 4.10f. The exact functional form for $\mathcal{A}(\omega, T)$ is

$$
\mathcal{A}(\omega, T)=e^{-g(2 N+1)} \sum_{l=0}^{\infty} \frac{g^{l}}{l !} \sum_{m=0}^{l}{ }_{l} C_{m} N^{m}(N+1)^{l-m} \delta\left(\omega-\epsilon_{0}+\Delta-(l-2 m) \omega_{0}\right)
$$

where ${ }_{l} C_{m}$ is combinatorial shorthand for " $l$ choose $m$ ", which is simply $\frac{l !}{m !(l-m) !}$. 

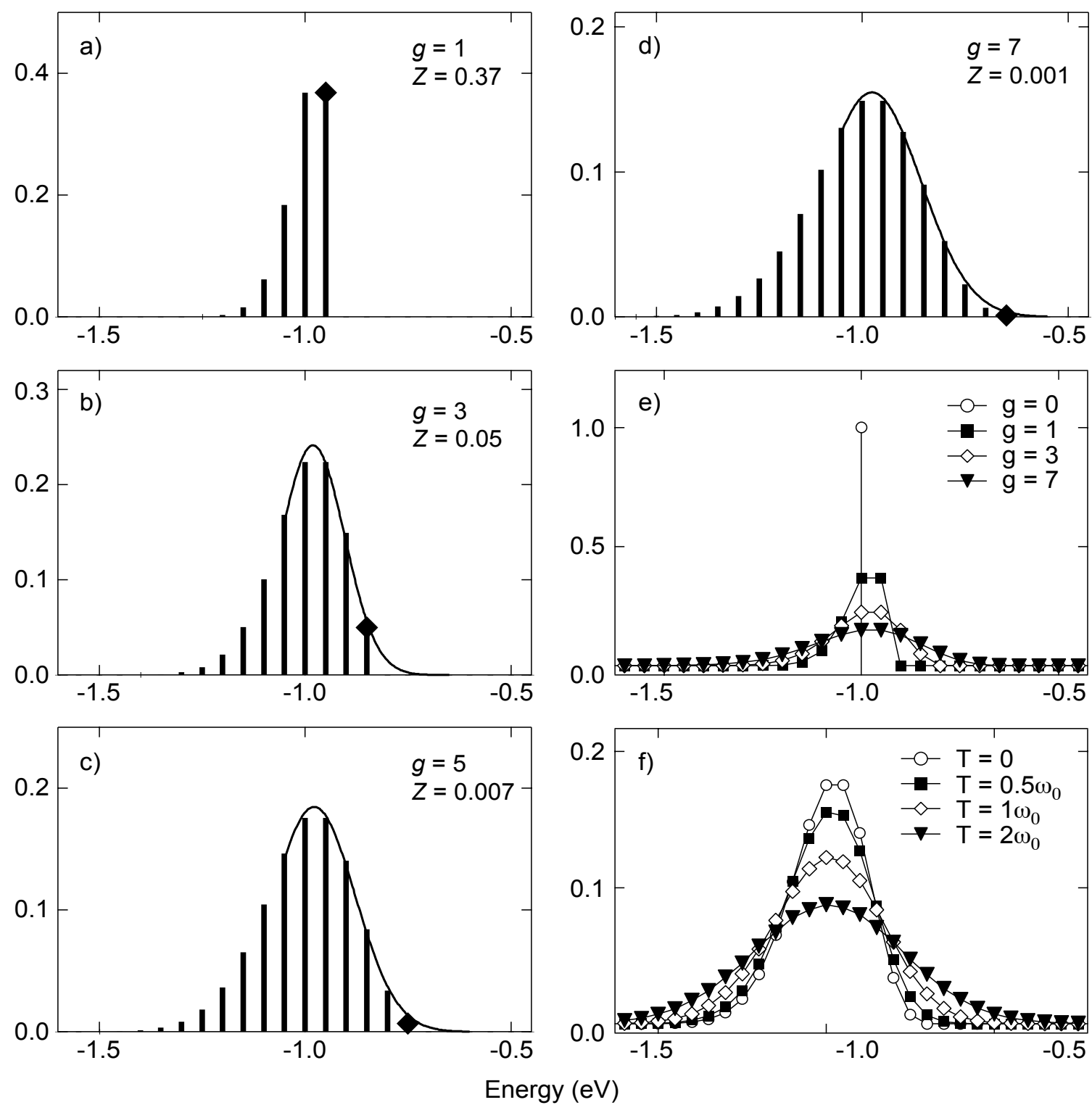

Figure 4.10: Calculations from the interacting electron-boson Hamiltonian, Equation 4.8. In this plot, we plot the case for a hole, not an electron, so that the energy axes are analogous to what one would observe in the photoemission process (as opposed to inverse photoemission). Panels (a)-(d) show the evolution of $\mathcal{A}(\omega)$ as a function of the electron-boson coupling constant, $g$. In this model, $\epsilon_{0}=-1 \mathrm{eV}$ and $\omega_{0}=0.05$ $\mathrm{eV}$. The position of the diamond shows the zero-phonon line $(Z)$, which would be analogous to the QP in the solid state. A fit of the lower binding part of $\mathcal{A}(\omega)$ to a Gaussian is shown as a thin solid line. A direct comparison of the spectral weight distribution as a function of $g$ is shown in (e). The evolution of $\mathcal{A}(\omega)$ as a function of temperature (measured in units of $\omega_{0}$ ) is shown in (f). 


\section{Chapter 5}

\section{Evidence for Lattice Polaron Formation in the Undoped Cuprates}

In Chapter 4, we demonstrated that using a phenomenology based on Franck-Condon broadening was far better suited to describing the single-electron excitations of the parent Mott insulator than the coherent quasiparticle scenario. This implies that the good eigenstates of the photoexcited $N-1$ system had very little overlap with noninteracting, free-electron-like states. In the photoemission process, we cannot measure the actual eigenstates of the system, only single electrons which must propagate roughly 1 meter through free space from the sample surface to our detector. Therefore, in a very real sense, we are measuring the projection of the eigenstates of the $N-1$ system onto the measurable free-electron plane-wave states. This line of thinking led us to the discussion of Franck Condon broadening in Chapter 4, where the eigenstates of that system have a strongly mixed electron-boson character. Because of this, it is more appropriate to refer to these eigenstates as "polarons" - i.e. an electron strongly coupled to a field of bosons ${ }^{1}$.

In the case of the undoped cuprates, it is rather difficult to determine a priori what

\footnotetext{
${ }^{1}$ The majority of the work discussed in this chapter is currently submitted as a manuscript to Physical Review B.
} 


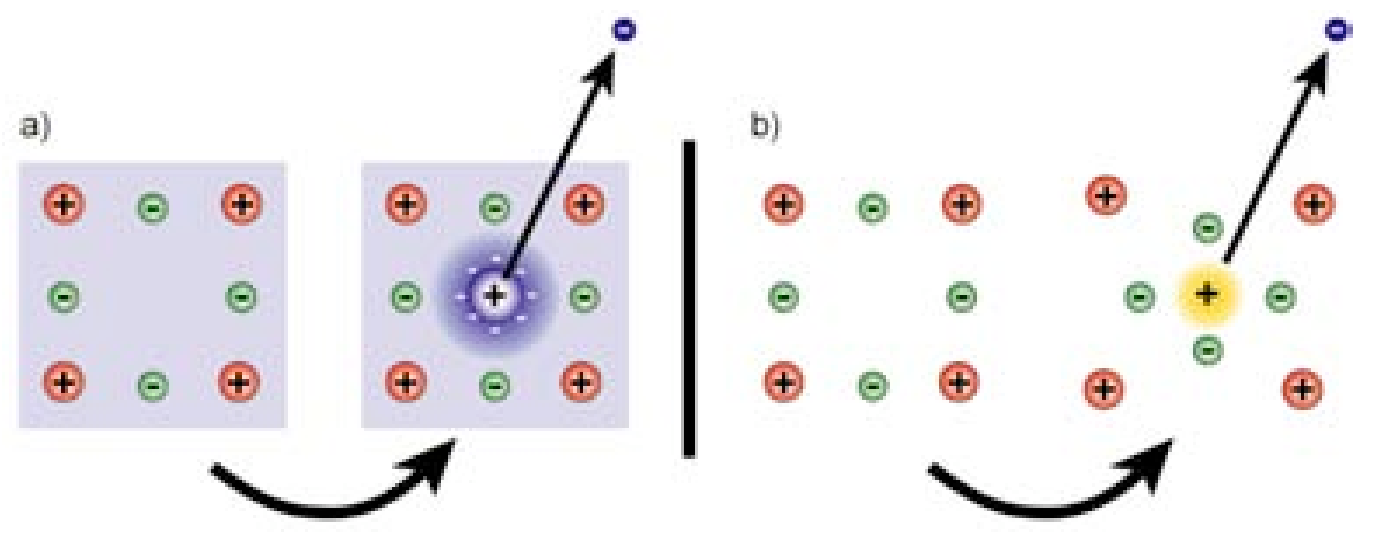

Figure 5.1: (a) Photoemission from a metal. Due to screening of the positively charged photohole by the conduction electrons, the lattice relaxation is small. (b) Photoemission from an ionic insulator. Without readily mobile screening electrons, the lattice ions are free to relax due to strong Coulomb interactions with the unscreened, positively charged photohole.

particular kind of bosonic field the electrons are coupling to. The idea of polarons first originated in the 1950's from the consideration that electrons could be strongly coupled to the lattice $[133,134,135,136]$. However, because the undoped cuprates are also antiferromagnetic, the photohole (which is a spin- $1 / 2$ object) can also be dressed by magnons, and therefore the injected photohole can also decay into a "spin polaron", i.e. a hole strongly dressed by a cloud of spin wave excitations. The objective of this chapter is to attempt to determine what mechanisms could cause this broadening. Because excitations from the LHB can, in principle, be coupled to the charge, spin, and lattice sectors, it is essentially impossible to disentangle each contribution. However, we can attempt to determine whether any lattice contribution to the FCB exists in the LHB.

In the case of lattice polaron formation, electronic localization is associated with the hole's $+e$ charge and its interactions with surrounding ions. Because the undoped system is an insulator, the neighboring ions should be poorly screened from the Coulomb interaction of the positively charged hole, as opposed to the case of a metal, where screening from the other conduction electrons (i.e. associated with plasmons) greatly weakens the effective photohole-lattice Coulomb interaction. A cartoon 
picture of this is shown in Figure 5.1. In the case of the oxychloride materials, it is generally accepted that the $\mathrm{CaCl}$ (or $\mathrm{SrCl}$ ) blocking layers are essentially ionic, and the positions of the $\mathrm{Ca}^{2+}$ cations or $\mathrm{Cl}^{-}$anions, or the copper or oxygen atoms within the plane, may relax in response to the electrostatic interaction with the photohole. This lattice relaxation results in the formation of a localized lattice polaron, which in a quantum-mechanical sense, is a hole dressed by a number of virtual phonons.

The basic concept of the spin polaron is analogous to a lattice polaron, and in discussed in detail by Shastry, Auerbach, and others [137, 138, 139, 140]. Because the hole carries both charge $+e$ and spin- $1 / 2$, its hopping motion through the lattice will also disrupt the antiferromagnetic spin sublattice. The motion of the hole therefore results in spin-flip processes, and the magnetic energy cost of the hole's propagation scales roughly with the distance that the hole has moved. Therefore, the energy cost of these spin-flip processes associated with the hole motion will favor a more localized photohole. Therefore, it may be energetically more favorable, in terms of the magnetic exchange energy, for the hole to form a small local region of ferromagnetically polarized spins in the antiferromagnetic background, and this massive "spin polaron" can then move coherently by tunneling, similar to a lattice polaron. A small lattice polaron state can be rather easily created, since an electron can be dressed by any number of phonons. On the other hand, it is unclear exactly how "small" a spin polaron can be created around an electron or hole. This is because magnon (spin flip) excitations will eventually bring the spin orientation back into its original direction, thereby putting a bound on the maximum number of magnons that can dress an electron, and this was the basis for some of the calculations performed by Mishchenko and Nagaosa [141] which will be discussed later in this chapter. In systems where orbital ordering occurs there exist bosonic modes, sometimes called "orbitons", associated with the broken symmetry associated with the orbital order. As a result, in such orbitally ordered systems, orbital polarons can also be formed [142, 143]. In the cuprates, this is irrelevant as the $\mathrm{Cu} 3 d_{x^{2}-y^{2}}$ orbital is the only unfilled $3 d$ orbital, so no such orbital degeneracy necessary for orbital ordering exists. However, these considerations might be essential for materials such as the manganites.

To complicate the situation, no detailed theoretical understanding of the spectral 
properties of the electronic Mott insulator currently exists. Because of the strongly correlated nature of the Mott insulating state, it is not known whether well-defined sharp quasiparticle-like excitations (which exist in the Fermi liquid state) would exist for the Mott insulator, irrespective of additional lattice or spin polaron effects. Some theoretical studies of the two-dimensional Hubbard model which focus on the effects of the electronic correlations on the single-particle properties will also be discussed along these lines. However, we can still attempt to determine whether lattice effects are, at any level, relevant to the observed Franck-Condon broadening in the undoped insulator. To do this, we compare the complex strongly correlated states in the lower Hubbard band (LHB) to conventional "benchmark" states (O $2 p_{\pi}$ and Ca $3 p$ orbitals). From this comparison, we conclude that lattice effects play a substantial role in the observed broadening, implying that the photoholes form localized small polaron states. In addition, we have observed a large momentum dependence to the linewidth which may have implications on the anisotropy of the first doped QP states in the lightly doped cuprates. This evidence of a strong lattice polaron effect still does not rule out additional contributions from spin or charge degrees of freedom to the lower Hubbard band.

\subsection{Photoemission Studies of Small Polaron Systems}

In order to discuss the possibilities of lattice polaron formation in the cuprates, we first provide a brief overview of previous photoemission studies of systems exhibiting small polaron behavior. Establishing this framework will be very important in order to conclusively demonstrate that lattice effects unequivocally contribute to the FranckCondon broadening observed in the undoped cuprates, as will be later described. We should note that while lattice-induced FCB / polaron formation has been known for over 30 years, there has not yet been (to our knowledge) any observation by photoemission of spin polaron formation. This certainly does not exclude the fact that spin polarons can be observed by photoemission, but the precedent for this has 

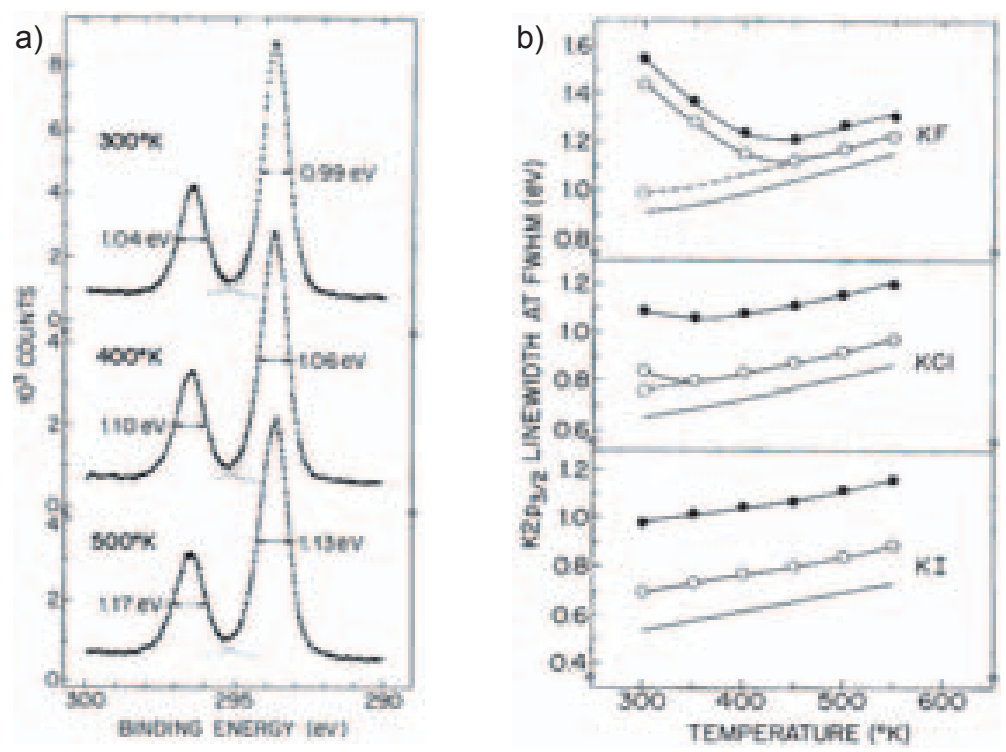

Figure 5.2: XPS spectroscopy of the ionic potassium halides, taken from [145]. (a) Temperature dependence of the EDCs of the K $2 p$ core level from KI. (b) Temperature dependence of the Gaussian FWHM of the core levels. The upturn at low temperatures is from electrostatic charging of the insulating samples.

not yet been set. However, we should note that recent work on the $\mathrm{Fe}(110)$ surface has claimed to observe electron-magnon effects in the electronic self-energy extracted by ARPES, although these effects appear to be rather subtle and not definitive [144]. There have been many claims that the observed dispersion anomalies in the cuprates are due to interactions between electrons and spin interactions [40], although this is still a highly controversial and unresolved topic. In principle, however, there is still no reason why such effects could not be observed by ARPES.

Much of the work presented in this chapter was based on the work of Citrin, Wertheim, and Baer $[145,146,147]$ on core-level broadening in simple alkali halides. In these very simple ionic systems, one does not need to be concerned about spin degrees of freedom, so any electron-boson interactions would most certainly be due to phonons (in this case, we neglect plasmons because of the vastly different energy scales of phonons and plasmons). In Figure 5.2a, we show core level spectra from the K $2 p$ electrons from KI taken from [145]. Two aspects of Figure 5.2a are critical to the identification of lattice FCB as the predominant source of broadening in the 
spectra. First, even in spite of the poor instrumental resolution, the lineshape is clearly Gaussian-like. This is significantly different from the Lorentzian-like DoniachŠunjić (DS) lineshapes observed in conventional metals such as Na or Al [148], where the predominant lifetime effects are due to the interaction of the conduction electrons with the localized core hole. Second is the sizable temperature broadening observed in Figure 5.2a and summarized in Figure 5.2b; the magnitude of the temperature broadening observed in the core levels of these alkali halides is substantially larger than what one would expect from electron-hole interactions, and again points to the coupling of the core hole to a low-energy bosonic field.

More recent photoemission work has centered on studies of lattice polaron effects on the near- $E_{F}$ valence electrons. A substantial amount of photoemission work on the colossal magnetoresistance (CMR) manganites has also demonstrated broad and incoherent, yet dispersive spectral weight with very little intensity at $\mathrm{E}_{\mathrm{F}}[149,150]$. This appears to be qualitatively very similar to the ARPES spectra observed in the undoped cuprates, and it is generally accepted that Jahn-Teller polarons play a critical role in the physics of the manganites [151], and the effects of small polaron formation in these systems have already been discussed [152]. This type of analysis has been extended to work for other transition metal oxides, including the vanadates [153, 154] and magnetite [155]. It is rather interesting to note an acknowledgement in one of Holstein's first papers on small polarons in 1959 [135] that it was experimental studies on transition-metal oxides that provided the initial stimulus for his own work. In addition to work on the transition-metal oxides, recent ARPES studies by Perfetti et al. of one-dimensional charge-density-wave systems such as the classic "blue bronze" $\mathrm{K}_{0.3} \mathrm{MoO}_{3}$ as well as $\left(\mathrm{TaSe}_{4}\right)_{2} \mathrm{I}$ seem to suggest that small polaron formation is also relevant in these strongly pseudogapped 1D Peierls systems $[156,157]$. This is rather different than the conventional textbook weak-coupling, FS nesting-driven scenario for CDW formation, but seems to provide a very appealing and self-consistent picture for understanding the spectral features of these 1D Peierls compounds. The sum total of this work on transition-metal oxides and 1D Peierls compounds would strongly suggest that lattice polaron effects are clearly exhibited also in the low-energy (near$E_{F}$ ) single electron excitations in ARPES. 


\subsection{Lattice Polaron Formation in $\mathrm{Ca}_{2} \mathrm{CuO}_{2} \mathrm{Cl}_{2}$}

\subsubsection{Polaron Formation in $\mathrm{O} 2 p_{\pi}$ States}

As described earlier, the complex many-body nature of the states in the LHB makes it extremely difficult to determine whether the large broadening arises from electronphonon, electron-magnon, or purely electron-electron effects. Therefore, it is important to have some comparison between the LHB states and other states in the crystal which do not have such complicated many-body interactions. We employ the logic that while many electronic states are not coupled to spin and electronic correlations in the $\mathrm{Cu} 3 d$ and ZRS states, all states must necessarily be coupled to the underlying lattice. Therefore, by selecting particular "uncorrelated" electronic states, we can determine whether these states experience an appreciable electron-lattice interaction. Although the electron-phonon interaction will obviously vary significantly between states, this should at least provide us with an estimate of how relevant electronphonon interactions may be to the broadening observed in undoped $\mathrm{Ca}_{2} \mathrm{CuO}_{2} \mathrm{Cl}_{2}$.

We start by using the $\mathrm{O} 2 p_{\pi}$ state identified in Chapter 4 as a reference state to compare against the LHB. A very similar comparison was first performed by Pothuizen et al. in $\mathrm{Sr}_{2} \mathrm{CuO}_{2} \mathrm{Cl}_{2}$ using the same aforementioned logic [126]. Our work adopts the same line of reasoning, but extends upon this work with a discussion of FCB, detailed lineshape analysis of high-resolution spectra, as well as with comprehensive temperature dependence data. As discussed by Pothuizen et al. [126] and later by Hayn et al. [125], at $\mathbf{k}=(\pi, \pi)$ this state has $100 \% \mathrm{O} 2 p_{\pi}$ character (as shown in Figure 4.3c) and therefore does not overlap with the $\mathrm{Cu} 3 d$ spin system. Therefore, the only mechanisms for the decay of a photohole injected into the $\mathrm{O} 2 p_{\pi}$ band at $\mathbf{k}$ $=(\pi, \pi)$ should be electron-hole and electron-phonon interactions. In principle, one can distinguish between electron-hole and electron-phonon decay of the photohole by the characteristic lineshapes of each process. In Figure 5.3a, we show EDCs along the $(0,0)-(\pi, \pi)$ direction of the Brillouin zone for the LHB near $\mathrm{E}_{\mathrm{F}}$. As discussed earlier, the LHB exhibits the Gaussian FCB lineshape not only at $(\pi / 2, \pi / 2)$, but all over the Brillouin zone. As can be seen, the Gaussian FWHM also seems to be strongly momentum dependent, and this will be discussed later in this chapter. 


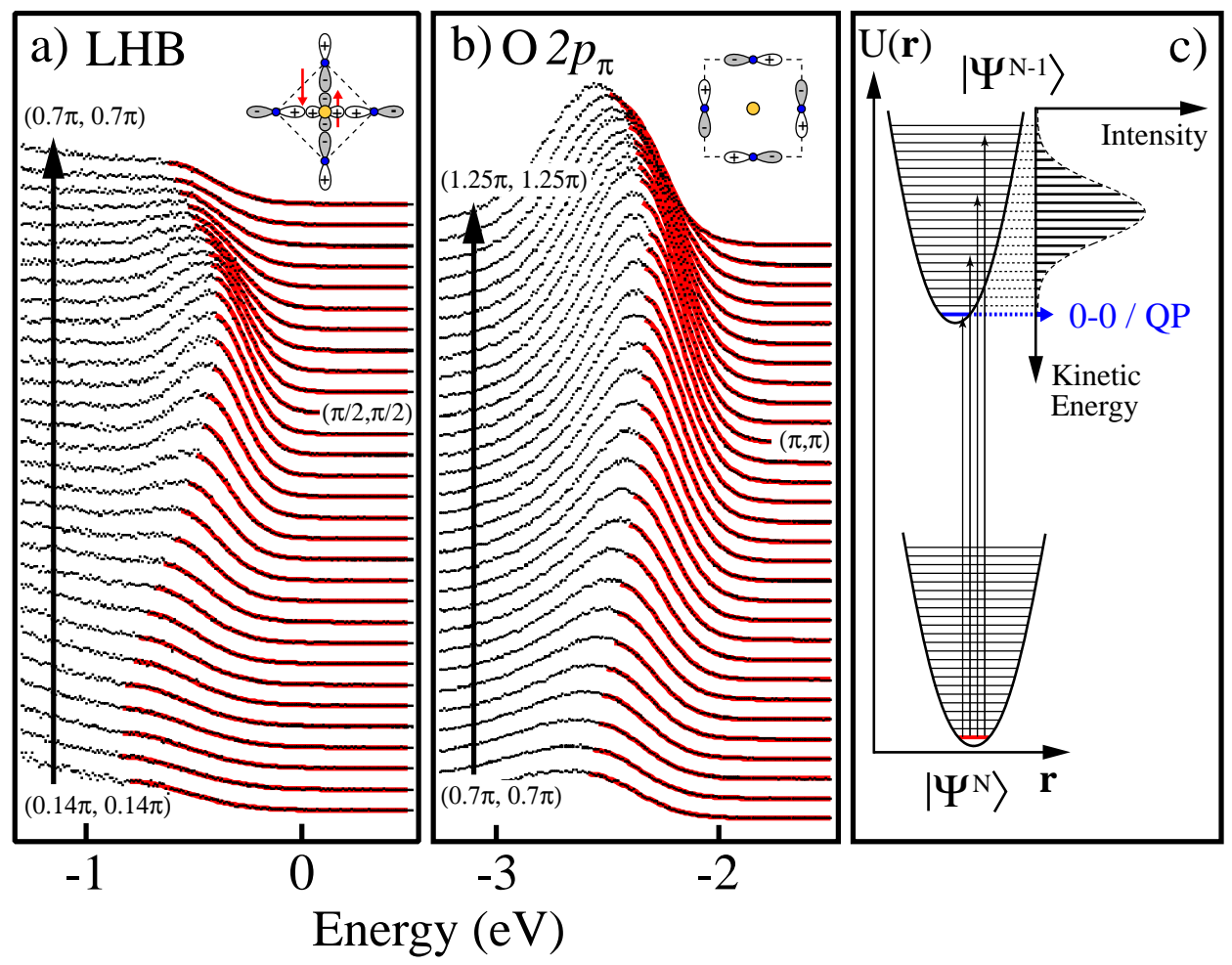

Figure 5.3: (a) Franck-Condon broadening of the LHB near $(\pi / 2, \pi / 2)$ along the $(0,0)-(\pi, \pi)$ direction. (b) Spectra from the O $2 p_{\pi}$ band near $(\pi, \pi)$ along the $(0,0)$ $(\pi, \pi)$ direction which also exhibit FCB. All raw data are shown as black points and Gaussian fits are shown as a thick red lines. Data were taken at $300 \mathrm{~K}$ with $h \nu=25.5$ $\mathrm{eV}$, and polarization at $45^{\circ}$ to the $\mathrm{Cu}-\mathrm{O}$ bonds. (c) Schematic of the Franck-Condon broadening process.

In Figure 5.3b, we show the EDCs from the O2p $p_{\pi}$ state near $(\pi, \pi)$. The main point is that the $\mathrm{O} 2 p_{\pi}$ peaks can also be fit very nicely with a Gaussian lineshape, similar to the LHB. Because electron-hole decay should result in an approximately Lorentzian lineshape, this is a very strong indication that the lineshape of the O $2 p_{\pi}$ states are also Franck-Condon broadened. Since the $\mathrm{O} 2 p_{\pi}$ states are not coupled to the spin degrees of freedom, this clearly implies that the photoholes injected into the $\mathrm{O} 2 p_{\pi}$ band form small lattice polarons, indicating the presence of very strong electron-phonon interactions for the $\mathrm{O} 2 p_{\pi}$ band in $\mathrm{Ca}_{2} \mathrm{CuO}_{2} \mathrm{Cl}_{2}$. This suggests that there might exist a sufficiently large electron-phonon interaction for states in the LHB to cause the observed FCB in $\mathrm{Ca}_{2} \mathrm{CuO}_{2} \mathrm{Cl}_{2}$. Therefore, our studies of the $\mathrm{O} 2 p_{\pi}$ 
lineshape in $\mathrm{Ca}_{2} \mathrm{CuO}_{2} \mathrm{Cl}_{2}$ have provided cause to believe that sizable lattice polaron effects should also exist for the LHB, and therefore may be largely responsible for the observed broadening. The Gaussian lineshape also seems to describe a fairly wide range of $\mathbf{k}$-space for the $\mathrm{O} 2 p_{\pi}$ band, much like the LHB.

First of all, electron-hole lifetime effects should also be relevant in the $\mathrm{O} 2 p_{\pi}$ band which sits $\sim-2.5 \mathrm{eV}$ below $\mathrm{E}_{\mathrm{F}}$. Assuming that this decay channel produces a Lorentzian lineshape, we can estimate the maximum contribution from electron-hole interactions by convoluting the Gaussian FCB lineshape with a Lorentzian. Estimating a total width at $\mathrm{T}=200 \mathrm{~K}$ of $370 \pm 50 \mathrm{meV}$, we estimate a maximum Lorentzian contribution of $\sim 70 \mathrm{meV}$ before significant deviations from a Gaussian lineshape. This upper bound for $\Gamma_{e l-e l}$ would correspond to an electron-phonon contribution of $\Gamma_{e l-p h}=300 \mathrm{meV}$, resulting in a Voigt parameter $y=\Gamma_{e l-e l} / \Gamma_{e l-p h} \sqrt{\ln 2}=0.19$. However, performing a more detailed fit of the Voigt parameters of the $\mathrm{O} 2 p_{\pi}$ lineshape is complicated by the high binding energy tail, which makes the $\mathrm{O} 2 p_{\pi}$ peak asymmetric. Calculations by Hayn et al. [125] predict a neighboring state of mixed $\mathrm{O} 2 p_{z} / \mathrm{Cu} 3 d_{x z, y z}$ character at $(\pi, \pi)-500 \mathrm{meV}$ away from the $\mathrm{O} 2 p_{\pi}$ state. If we assume that the observed broadening is entirely due to electron-phonon interactions, this will allow us to make an estimate of the electron-phonon coupling constant. The data yield a value of $\Gamma_{\pi}(200 \mathrm{~K})=370 \pm 50 \mathrm{meV}$, and using the independent boson model from Chapter 4, we find that the polaron binding energy is

$$
\Delta P=g \omega_{0}=\Gamma^{2} /\left(8 \ln 2 \omega_{0}\right)
$$

where $g$ is the electron-phonon coupling constant and is a dimensionless quantity related to the expectation value for the number of virtual phonons in the polaron cloud. However, our estimates of $\Delta P$ and $g$ depend rather sensitively on the choice of $\omega_{0}$. If we use $\omega_{0}=40 \mathrm{meV}$, which is the centroid energy of the LO oxygen phonons, we obtain $\Delta P=0.62 \pm 0.18 \mathrm{eV}$ and $g=15 \pm 5$, while if we use $\omega_{0}=70 \mathrm{meV}$ (the half-breathing mode), $\Delta P=0.35 \pm 0.1 \mathrm{eV}$ and $g=5 \pm 1.4$. Unfortunately, without $a b$ initio calculations, it would be difficult to estimate which particular phonon modes are most strongly coupled to a photohole in the $\mathrm{O} 2 p_{\pi}$ band. 


\subsection{Temperature Dependence of the Franck-Condon Broadening}

The data presented in the previous section provide unequivocal evidence that very significant coupling exists between the photohole and the lattice in $\mathrm{Ca}_{2} \mathrm{CuO}_{2} \mathrm{Cl}_{2}$. Another piece of evidence to support this view is the temperature dependence of the broadening. We have measured the temperature dependent width of the LHB, $\mathrm{O} 2 p_{\pi}$ and also Ca $3 p$ core levels. All three states exhibit a temperature dependent broadening much larger than one would expect from conventional electron-electron interactions, and are again consistent with the coupling of the electronic states to a low-energy bosonic field. Theoretical studies of the $t-J$ model have also shown temperature dependent broadening of the LHB $[158,130]$, but only at very large temperature scales $(\mathrm{T}=J / 2, J)$, while we have seen significant changes over even a small $(\sim 200 \mathrm{~K})$ range.

\subsubsection{Temperature Dependence of Valence Band States}

The temperature dependence of the FCB was treated earlier in Chapter 4 in the independent boson model, and is simply dependent on the Bose-Einstein distribution of thermally populated phonons. The temperature dependent spectral function $\mathcal{A}(\omega, T)$ is plotted in Figure 4.10f, and is expressed as an analytical function in Equation 4.11. The FWHM of the phonon sidebands can also be expressed as an analytical function [159], and applied by Citrin et al. for the potassium halides [145]. The temperature dependence of the width is given in Equation 5.2

$$
\Gamma(T)=2.35 \sqrt{\hbar \omega_{0} \Delta E}\left[\operatorname{coth}\left(\hbar \omega_{0} / 2 k T\right)\right]^{1 / 2}
$$

where $\omega_{0}$ is the phonon frequency and $\Delta E$ is the lattice relaxation energy. In a simplified model considering the interaction between a localized electronic state and an LO phonon, the expression for $\Delta E=e^{2}\left(6 / \pi V_{m}\right)^{1 / 3}\left(1 / \epsilon_{\infty}-1 / \epsilon_{0}\right)$, where $V_{m}$ is the volume of the primitive unit cell and $\epsilon_{\infty}$ and $\epsilon_{0}$ are the low- and high-frequency dielectric constants, respectively [160]. The applicability of Equation 5.2 and the 


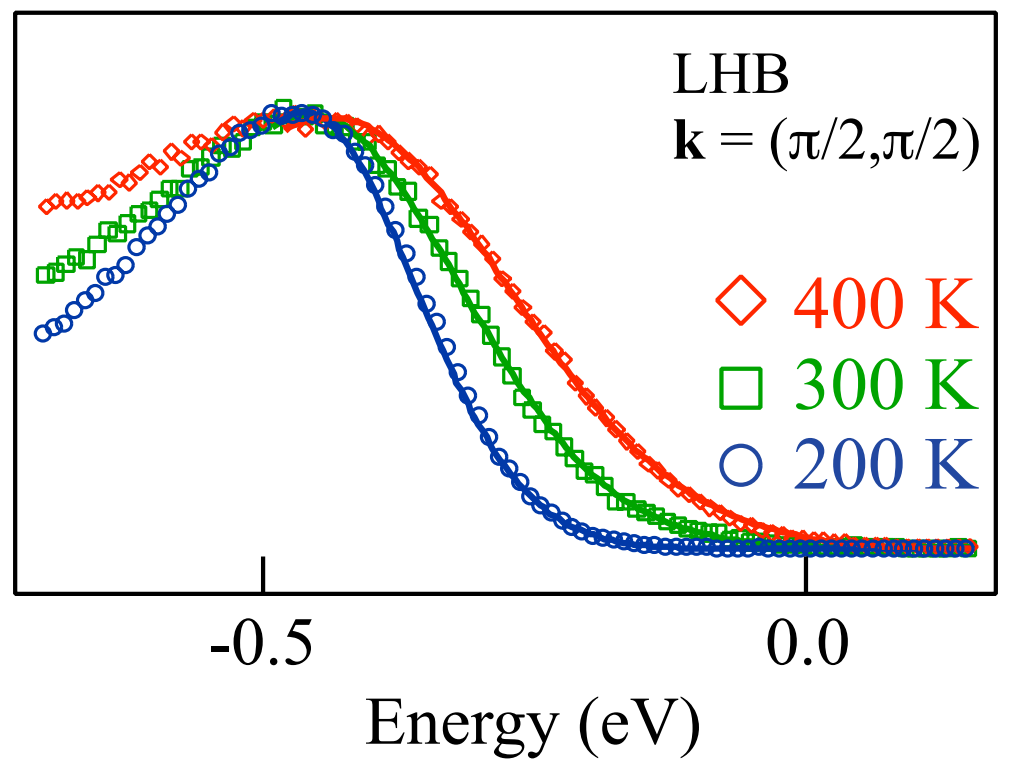

Figure 5.4: Temperature dependence of states in the lower Hubbard band from 200$400 \mathrm{~K}$ taken at $(\pi / 2, \pi / 2)$. All spectra are scaled and shifted so that all peak heights and positions match, as to best illustrate the temperature dependent broadening. Gaussian fits are shown as solid lines.

form for $\Delta E$ is probably quite good for a localized core level in the alkali halides. However, for delocalized valence electrons in the undoped cuprates (which have a much more complex unit cell than the alkali halides), the utility of these expressions is suspect. To our knowledge, there is no published information regarding $\epsilon_{\infty}$ or $\epsilon_{0}$ for $\mathrm{Ca}_{2} \mathrm{CuO}_{2} \mathrm{Cl}_{2}$. However, far more extensive optical conductivity work is available for $\mathrm{La}_{2-x} \mathrm{Sr}_{x} \mathrm{CuO}_{4}$, so we can use results from undoped $\mathrm{La}_{2} \mathrm{CuO}_{4}$ as a rough estimate for $\Delta E$ and $\Gamma(T)$. The ARPES spectra for the valence band and $\mathrm{LHB}$ in $\mathrm{La}_{2} \mathrm{CuO}_{4}$ look substantially different from $\mathrm{Ca}_{2} \mathrm{CuO}_{2} \mathrm{Cl}_{2}$. In $\mathrm{La}_{2} \mathrm{CuO}_{4}$, published data from Falck et al. [161] and Chen et al. [162] give values of $\epsilon_{\infty}=5$ and $\epsilon_{0}=30$, respectively. For $\mathrm{La}_{2} \mathrm{CuO}_{4}$, this would give a $\Delta E=6.5 \mathrm{eV}$ and a $\Gamma(0) \sim 1.2 \mathrm{eV}$. Quite clearly, this is a gross overestimate of the nuclear relaxation energy and $\Gamma(0)$. However, the relevance of these expressions for valence electrons in a complex material like the cuprates should not be high.

In Figure 5.4, we show the temperature dependence of the LHB at $(\pi / 2, \pi / 2)$; the peak position and peak height are all normalized to the same value, emphasizing the 


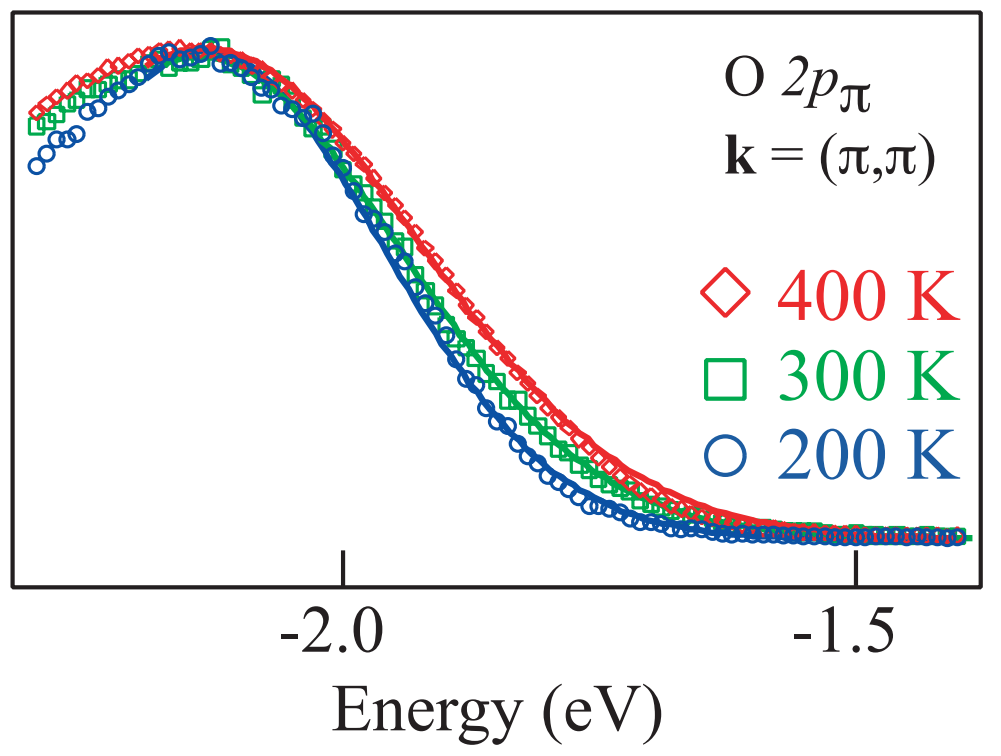

Figure 5.5: Temperature dependence of states in from the O2p $p_{\pi}$ band from 200-400 K taken at $(\pi, \pi)$. All spectra are scaled and shifted so that all peak heights and positions match, as to best illustrate the temperature dependent broadening. Gaussian fits are shown as solid lines.

observable change in width. The peak widths for the LHB change quite dramatically as a function of temperature, as can be seen easily in the raw data. Along with the data are Gaussian fits (solid lines) to the low-binding energy half of the peaks; the Gaussian lineshape seems to fit the experimental spectra well at all three temperatures. We were unable to obtain a reliable temperature dependence of the pinned position of $\mu$ as a function of temperature. This was primarily because $\mu$ is sensitive to surface defects and adsorbates. For instance, $\mu$ could be changed irreversibly by cycling the temperature or introducing a brief pressure burst to the chamber. Although there was still a sizable scatter from sample to sample, the FWHM behaved more reliably than $\mu$ as a function of temperature, so we will only discuss the temperature dependence of the FWHM. In Figure 5.5, we show the temperature dependence of the $\mathrm{O} 2 p_{\pi}$ state at $(\pi, \pi)$, where the peak positions and heights are likewise normalized as in Figure 5.4. The $\mathrm{O} 2 p_{\pi}$ peak is also well fitted by a Gaussian lineshape (solid lines) at all three temperatures, although the temperature dependent broadening is less dramatic than that observed in the LHB. 


\subsubsection{Temperature Dependence of $\mathrm{Ca} 3 p$ Core Levels}

In Figure 5.6a, we show a wide-range valence band scan of $\mathrm{Ca}_{2} \mathrm{CuO}_{2} \mathrm{Cl}_{2}$ using $\mathrm{He}$ II radiation (from the Gammadata VUV5000). With $h \nu=40.8 \mathrm{eV}$, we can reach the $\mathrm{Ca} 3 p$ core levels which sit at $\sim-19 \mathrm{eV}$ binding energy. These core levels are highlighted in red. Like the $\mathrm{O} 2 p_{\pi}$ band, the Ca $3 p$ core levels are obviously decoupled from the complex many-body interactions of the LHB, and therefore any evidence for FCB in the Ca $3 p$ states should also be taken as evidence for strong electron-phonon interactions. In Figure 5.6b, we show the Ca $3 p$ states in Figure 5.6a on an expanded scale after the subtraction of a Shirley-like background. Although the widths of the Ca $3 p$ states is large, clear temperature dependent broadening can be observed over a modest temperature range. At $-19.5 \mathrm{eV}$, there is a small dip between the $3 p_{3 / 2}$ and $3 p_{1 / 2}$ peaks which becomes more pronounced upon lowering the temperature. Even on this large energy scale, a change of $\sim 100 \mathrm{~K}$ is enough to produce a discernible change in the raw spectra. To extract quantitative widths, we perform a detailed fit of the Ca $3 p$ core levels.

Upon closer inspection, the Ca $3 p$ core levels are comprised of two spin-orbit split doublets, resulting in the small shoulder at $\sim-17.8 \mathrm{eV}$. We ascribe the second doublet to a surface core level shift (SCLS) of $\mathrm{Ca}^{2+}$ in the ionic $\mathrm{CaCl}$ cleavage plane arising from the altered Madelung potential from the surface termination. A SCLS is common in many materials, including cuprates such as $\mathrm{YBa}_{2} \mathrm{Cu}_{3} \mathrm{O}_{7-\delta}$ [163], and does not necessarily affect the electronic states in the underlying $\mathrm{CuO}_{2}$ plane. Through a simple analysis using the known structure and taking the escape depth as $10 \AA$ [163], we obtain the surface intensity fraction to be $S_{f}=0.31$, roughly consistent with experiment. Even without fitting, the raw data exhibit observable temperature broadening, as evidenced by the dip at $-19.5 \mathrm{eV}$ (arrow) becoming more pronounced at lower temperatures. Fits to a pair of Gaussian doublets are shown, where the parameters $S_{f}(0.33)$, spin-orbit splitting $(1.19 \mathrm{eV})$, and the SCLS $(0.61 \mathrm{eV})$ were kept fixed with temperature. Two doublets in the Ca $2 p$ core levels were also observed using XPS by Koitzsch et al. [164] with a separation of $\sim 1 \mathrm{eV}$. In this work, they ascribed the second Ca $2 p$ doublet to the presence of Ca defects and vacancies of the topmost $\mathrm{CaCl}$ layer. However, the intensity of their second doublet would imply that 

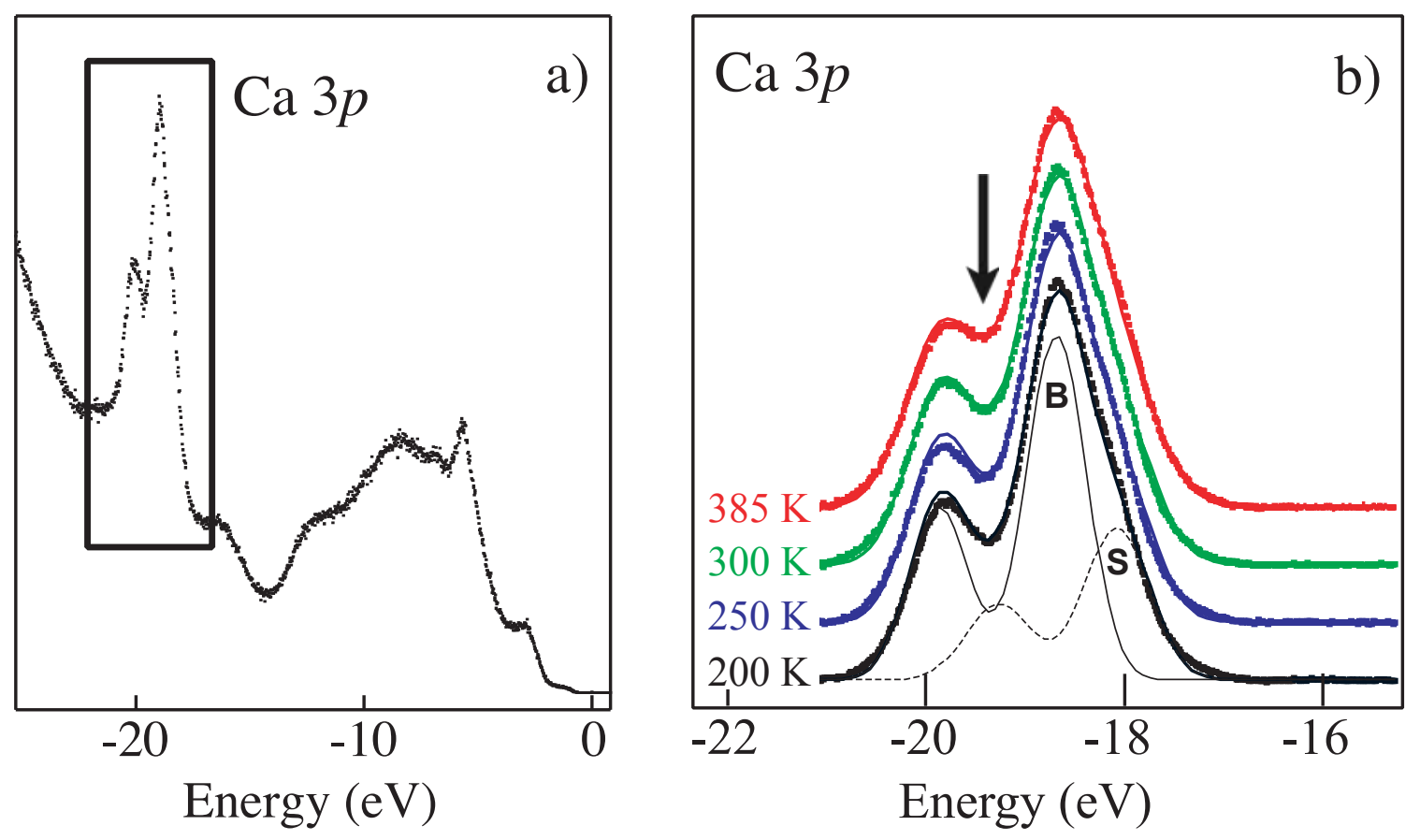

Figure 5.6: (a) Raw data over a wide energy range from $\mathrm{Ca}_{2} \mathrm{CuO}_{2} \mathrm{Cl}_{2}$ taken using $\mathrm{He}$ II radiation, showing the Ca $3 p$ core levels (outlined). (b) Spectra from the Ca $3 p$ core level taken using He II radiation on an expanded scale. A Shirley-like background was subtracted from the raw data in (a) to give the spectra in (b). Fits to a pair of doublets are shown as solid lines, and a detailed discussion of the fits is given in the text. Bulk and surface core levels are shown in dotted lines as "B" and "S", respectively.

approximately $25 \%$ of the Ca atoms would be vacant. This number is unreasonably high, and certainly not borne out by STM studies of $\mathrm{Ca}_{2-x} \mathrm{Na}_{x} \mathrm{CuO}_{2} \mathrm{Cl}_{2}$ which show 1-2 orders of magnitude fewer vacancies $(\sim 1 \%)$ in the $\mathrm{CaCl}$ plane than suggested by Koitzsch et al..

The relatively good agreement of the Gaussian lineshapes used in Figure 5.6b would again suggest lattice-induced FCB. However, because of the uncertainties involved in the background subtraction, as well as the complex (four-peak) spectra of the $\mathrm{Ca} 3 p$ core levels, it is difficult to make any definitive statements as to the precise lineshape and the amount of (Lorentzian) electron-electron contribution to the photohole lifetime. However, it is quite clear that any temperature dependence of 
the core level width (especially within a $200 \mathrm{~K}$ window) should most likely arise from electron-lattice interactions. Therefore, any temperature-independent offset to the measured width from electron-electron interactions will result in an underestimate of the temperature dependence of the electron-phonon interactions, so $d \Gamma / d T$ from our extracted data should represent a lower bound.

\subsubsection{Temperature Dependence of Franck-Condon Broaden- ing}

In Figure 5.7, we show a summary of the temperature dependence of the width of all three peaks. We found a sizable sample-to-sample scatter in the FWHM of the $\mathrm{O} 2 p_{\pi}$ and LHB peaks. The average values of $\Gamma(T)$ are shown as large symbols with representative error bars, while individual measurements are shown as small symbols. Despite sample-to-sample variations, the data in Figure 5.7 clearly establish a temperature dependent broadening. In addition, measurements performed on the same cleaved surface at multiple temperatures also support the trends shown. Reliable and reproducible temperature dependent measurements were difficult to carry out primarily due to sample aging due to surface adsorbates arising from outgassing or cooling of the sample manipulator. In addition, measurements could only be performed typically above $\sim 180 \mathrm{~K}$ due to the onset of electrostatic charging of the sample as undoped $\mathrm{Ca}_{2} \mathrm{CuO}_{2} \mathrm{Cl}_{2}$ becomes more insulating at lower temperatures. In order to perform more extensive temperature dependent experiments, better vacuum conditions for temperature cycling and reducing sample charging at low temperatures will be necessary.

Along with the raw data in Figure 5.7, we also show fits to Equation 5.2, with $\omega_{0}$ and $\Delta E$ as free parameters in the fit, although we constrained $\omega_{0}>30 \mathrm{meV}$. For the Ca $3 p$ states, which should be most appropriate for Equation 5.2, $\Delta E=1.44 \mathrm{eV}$ and $\omega_{0}=60 \mathrm{meV}$. For the $\mathrm{O} 2 p_{\pi}$ states, $\Delta E=0.57 \mathrm{eV}$ and $\omega_{0}=37 \mathrm{meV}$, and finally for the LHB, $\Delta E=0.34 \mathrm{eV}$ and $\omega_{0}=30 \mathrm{meV}$ (the minimum constrained value for $\left.\omega_{0}\right)$. The agreement for the fit for the Ca $3 p$ states is quite good, and also reasonable for the $\mathrm{O} 2 p_{\pi}$ states. However, for the LHB, one finds the agreement to be poor, as 


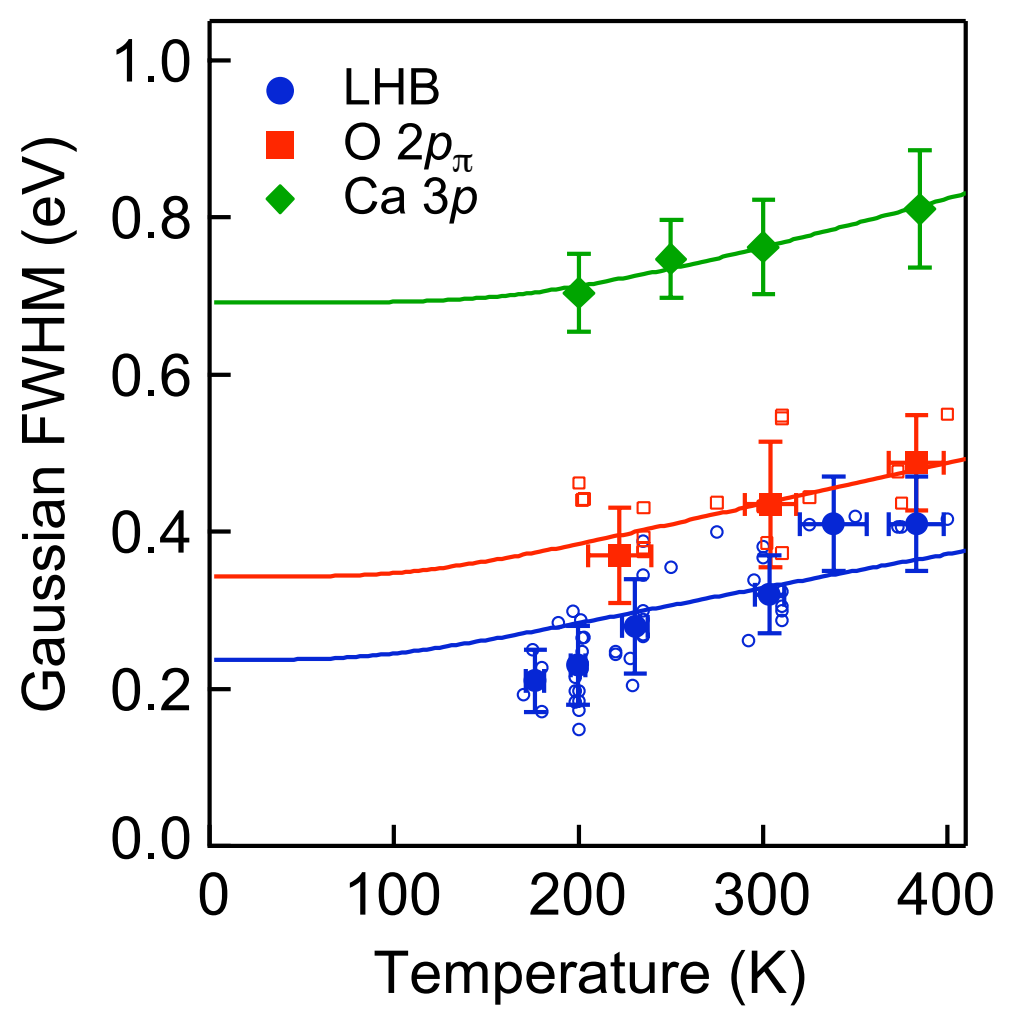

Figure 5.7: Summary of temperature dependent broadening of the LHB (circles), $\mathrm{O} 2 p_{\pi}$ (squares), and Ca $3 p$ (diamond) states. Small open symbols represent individual measurements, while the larger symbols represent the average values and error bars. Also, we show the fit of the experimental data to Equation 5.2.

the temperature dependence of the LHB broadening is much larger (and more linear) than one would expect from Equation 5.2. This might suggest a number of distinct possibilities. First, it is very plausible that the simple expression in Equation 5.2 is inappropriate for delocalized valence electrons within a complex crystal structure. Second, that electron-electron correlations or electron-magnon interactions introduce additional temperature dependent broadening terms beyond the simple lattice polaron picture. In fact, both possibilities seem very reasonable. Between $200<\mathrm{T}<400 \mathrm{~K}$, $d \Gamma / d T$ was estimated to be $1.0 \pm 0.3 \mathrm{meV} / \mathrm{K}$ for the LHB, $0.6 \pm 0.4 \mathrm{meV} / \mathrm{K}$ for the $\mathrm{O} 2 p_{\pi}$, and $0.7 \pm 0.2 \mathrm{meV} / \mathrm{K}$ for the Ca $3 p$ core level. In comparison, core level measurements of alkali halides such as $\mathrm{KCl}$, where polaron formation is also observed, 
give comparable values of $d \Gamma / d T(\sim 0.8 \mathrm{meV} / \mathrm{K})[145]$.

One potential concern with the strong temperature dependence of the LHB is that using the above value of $d \Gamma / d T=1.0 \pm 0.3 \mathrm{meV} / \mathrm{K}$, this would extrapolate to $\Gamma(0)$ $=0 \mathrm{eV}$. Although Equation 5.2 shows that $\Gamma(T)$ should saturate at low temperatures, sample charging makes such low temperature experiments nearly impossible. However, we have reason to believe that $\Gamma_{L H B}(T)$ does, in fact, saturate at low temperatures and that the width does not shrink to zero at low temperatures. First, a single set of early data taken by Filip Ronning showed that upon cooling from 180 $\mathrm{K}$ to $20 \mathrm{~K}$, there was no appreciable change in $\Gamma_{L H B}\left(\Gamma_{L H B}(20) \sim \Gamma_{L H B}(200) \sim 200\right.$ meV). However, in this measurement, no checks were made of the effects of sample aging or slight electrostatic charging at $20 \mathrm{~K}$, and this very difficult low temperature measurement has never since been reproduced. However, we can speculate based on these measurements that $\Gamma_{L H B}(0) \sim \Gamma_{L H B}(200)$ for the LHB. Additional evidence comes from nominally undoped $\mathrm{Nd}_{2} \mathrm{CuO}_{4}$ [26, 27], where measurements of the LHB were performed at $10 \mathrm{~K}$ and no evidence of charging was found for these samples. These measurements suggest that $\Gamma_{L H B}(T)$ does saturate at low temperatures, and that the FCB is not simply associated with thermally populated phonons.

\subsubsection{Possible Extrinsic Broadening Factors}

As the actual QP (or 0-0 transition) in small polaron systems is undetectable, the primary experimental evidence for polaron formation comes from the lineshape and linewidth of the incoherent multi-boson sidebands. Therefore, excluding potential extrinsic broadening factors is critical. Here, we discuss and rule out possible broadening factors unrelated to polaron formation.

One potential source of lattice-induced broadening unrelated to polaron formation is a Doppler broadening effect - the recoil energy that the ejected photoelectron imparts to the ion. Clearly, the magnitude of the Doppler broadening should be proportional the momentum of the outgoing photoelectron; at the reasonably low kinetic energy of our UPS experiments, this recoil effect should be rather small. The FWHM of this recoil broadening has been given by Flynn [165] as 


$$
\Gamma_{\text {rec }}=\sqrt{8 \ln 2}\left(\frac{P^{2} k T}{M_{0}}\right)^{1 / 2}
$$

where $P$ is the photoelectron momentum and $M_{0}$ is the ionic mass. If $k T<\epsilon_{0}$, where $\epsilon_{0}$ is the zero-point energy of the solid (which we can estimate as $\frac{1}{2} \hbar \omega_{0}$ ), we replace $k T$ by $\epsilon_{0} / 2$. For the Ca $3 p$ core level using He II radiation at $400 \mathrm{~K}$, this gives a $\Gamma_{\text {rec }}=10 \mathrm{meV}$, while for the LHB at $200 \mathrm{~K}$ using $25.5 \mathrm{eV}$ and taking the oxygen mass, this would give $\Gamma_{\text {rec }}=12 \mathrm{meV}$. Added in quadrature to an intrinsic width of $400 \mathrm{meV}$, this contribution is essentially negligible, and therefore not relevant to our experimental measurements. However, taking into account the zero-point energy of the lattice $\epsilon_{0} \sim 0.02 \mathrm{eV}$ and assuming that the low-energy states in the metallic cuprates are primarily oxygen-derived, this would give a lower limit of $\Gamma_{\operatorname{rec}(T=0)}$ (at $h \nu$ $=25 \mathrm{eV}$ ) of $\sim 8 \mathrm{meV}$. This value is well within the accessible range of today's electron detectors, and it would be interesting to observe whether Doppler recoil energies may present a practical obstacle to high-resolution experiments on metallic cuprates (and other transition-metal oxides), even at intermediate photon energies.

Another possible consideration is ohmic losses of the photoelectron as it leaves the solid, as proposed by Joynt [166] to be relevant to poorly conducting metals. For the case of an insulator, however, a lack of mobile conduction electrons should make such losses irrelevant. Nevertheless, even if we ignored this point, these ohmic losses should depend sensitively on the momentum of the outgoing photoelectron. Therefore, if we believed that the Gaussian lineshapes in the cuprates were affected by ohmic losses, the shape of the spectra themselves should be highly dependent on the outgoing photoelectron energy, and hence on the incident photon energy, $h \nu$, and is easy to check for, experimentally. Our experiments spanning the range from 15-30 $\mathrm{eV}$ show no appreciable change in the Gaussian FWHM of the LHB, and therefore we can rule out the effect of any ohmic losses. Also, later work by Schulte et al. [167] also seemed to suggest that the effects of ohmic losses even in poor conductors, such as the CMR manganites, were much less pronounced than predicted by Joynt [166]. We should point out that the photoelectron matrix element of the LHB varied very strongly with incident photon energy, but the measurable FWHM still did not 
change. Moreover, this photon energy invariance of the FWHM would also rule out other possible concerns, such as the Doppler broadening effect mentioned above, or breakdowns of the sudden approximation or three-step model.

In semiconductor physics, a Gaussian tail in the DOS, a so-called Urbach tail [168], is often associated with related to a distribution of impurity states. In any insulator or semiconductor, defect and impurity states will have some impact on the total DOS. $\mathrm{Ca}_{2} \mathrm{CuO}_{2} \mathrm{Cl}_{2}$ and $\mathrm{Sr}_{2} \mathrm{CuO}_{2} \mathrm{Cl}_{2}$ are the most ideal undoped cuprates because the stoichiometric compounds are Mott insulators. Therefore, the number of defects (from cation cross-substitution [108]), vacancies, or impurities should be rather small, less than $1 \%$. However, we should also consider the existence of surface defects or vacancies introduced in the cleavage process; STM measurements estimate the density of surface vacancies [169] to be on the order of $\sim 1 \%$. However, the Gaussian fit of the LHB extends all the way to the peak of the LHB, and should not be considered just a very small tail in the DOS. Therefore, a small concentration of defects $(\sim 1 \%)$ should not account for such a large amount of spectral weight encompassed in the Gaussian. Moreover, this impurity tail should not be strongly temperature or momentum independent. However, we find that the peak position disperses very strongly with momentum, and also the FWHM is strongly momentum dependent; this would not be consistent with a dilute distribution of impurity states. The large temperature broadening of the LHB would also argue against impurity states, whose temperature dependence should depend only on the Fermi-Dirac distribution. Moreover, we obtain sharper, low-energy structures (well-defined peaks, shown in Chapter 6) in energy and momentum at higher doping levels where the impurity concentration is much higher, suggesting that impurity scattering effects are not primarily culpable for the broad peaks observed in the insulator

Perhaps the most insidious of all extrinsic broadening effects is that of electrostatic sample charging. Charging in insulators occurs when the photocurrent exceeds the rate at which electrons can be replenished (from ground) to the sample surface, thereby resulting in a net positive charge buildup on the surface. Accurate, highresolution ARPES studies of insulators are notoriously difficult due to such charging effects. Moreover, charging cannot be entirely excluded, since it is certainly present 
at low enough temperatures. Two characteristics of electrostatic charging are a shift of the spectra to higher binding energies (due to the positive potential on the surface) and a overall broadening of the spectra (due to spatial inhomogeneities in the charging potential). It is highly likely that the broadening from inhomogeneous charging would take on a Gaussian distribution. To check for charging, we varied the incident photon flux to see whether the photoemission spectra exhibited any changes, since these effects should be dependent on the total photocurrent. We typically found no substantial changes above $\mathrm{T}=200 \mathrm{~K}$, although this temperature varied considerably between samples. We believe this was primarily due to the sample contact resistance and the sample thickness. However, it is conceivable that slight inhomogeneous charging might be responsible for some of the scatter among individual cleaves in Figure 5.7. Nevertheless, we note that the temperature dependent broadening observed would run contrary to the trends expected from charging; at higher temperatures, $\mathrm{Ca}_{2} \mathrm{CuO}_{2} \mathrm{Cl}_{2}$ becomes more conducting and therefore charging should become less important. However, the peak widths become sharper at lower temperatures, where charging should worsen. Therefore, we can conclude that electrostatic charging effects are not the primary source of the peak broadening observed. In addition, as will be discussed in Chapter 6, the wide Gaussian lineshapes of the $\mathrm{O} 2 p_{\pi}$ states are retained at finite doping levels (metallic or SC samples), where charging becomes irrelevant. Therefore, we can again conclude that the inherent widths of the peaks do not arise from sample charging.

\subsection{Theoretical Studies of Small Polaron Systems}

As discussed in Chapter 4, an analytical solution of the general case of electronboson interactions does not exist. Instead, the "toy model" Hamiltonian for the interacting electron-boson system assumed a single, nondispersive electronic state, and a single, nondispersive bosonic branch (an Einstein mode). In many solids, such as ionic insulators, the assumption of an Einstein mode may be a good approximation. However, the dispersion of the bare electronic state (in the absence of electron-boson interactions) poses a significant challenge to this model. As shown in the discussion 
of the IFB model, interactions with the bosonic field reduce the spectral intensity of the QP peak, $Z$, and result in a manifold of "bands" separated by the boson frequency, $\omega_{0}$, representing the "0-boson" line, the "1-boson" line, "2-boson" line, etc... Another consequence of the IFB model was that the electron-boson interactions did not change the first moment (i.e. the center of mass or centroid) of spectral weight, which remained peaked at the bare electronic energy, $\epsilon_{0}$.

For the case of a dispersing electronic state, $\epsilon(\mathbf{k}) \neq \epsilon_{0}$, it is unclear whether the conclusions of this simple model will continue to hold. For instance, will the first moment of spectral weight in the polaronic system track the bare electronic band $\epsilon(\mathbf{k})$ at all wavevectors, as one might naïvely expect? Will the "width" of this spectral envelope still be a measure of the electron-boson coupling strength? Although the answers to these questions have still not been conclusively determined, a number of theoretical treatments of this problem have shed some new light on this issue. Here we briefly outline two treatments of this problem. The first is based on very sophisticated quantum Monte Carlo calculations by A. Mishchenko and N. Nagaosa of the single hole in the $t-J$ model, with the addition of an Einstein phonon branch. This technique uses an exact diagrammatic Monte Carlo method where the phonon propagators are allowed to cross (i.e. allowing many phonon excitations around the hole - a necessary condition for small polaron formation). This may be the closest approximation to the real, physical situation of photoemission from the undoped parent cuprate. In fact, the results from these calculations, shown in Figure 5.8 from [141], were a large motivating factor for the Franck-Condon / small polaron analysis pursued in this thesis.

The primary result from these sophisticated calculations of the $t-J+$ phonon model is that the coherent "true QP" or zero-phonon line has vanishingly small weight and is nondispersive, while the centroid of incoherent weight follows the original dispersion defined in the original, frozen-lattice $t-J$ model. This apparent juxtaposition of polaronic QPs yet dispersive spectral weight allows us to "have our cake, and eat it too", so to speak. This is shown in Figure 5.8a, where the $\delta$-function is the zerophonon line, and the feature marked " $\mathrm{C}$ " is the $t-J$ band. The dispersion of the zero-phonon line and the centroid (C) is shown in Figure 5.8b, where the quantum 

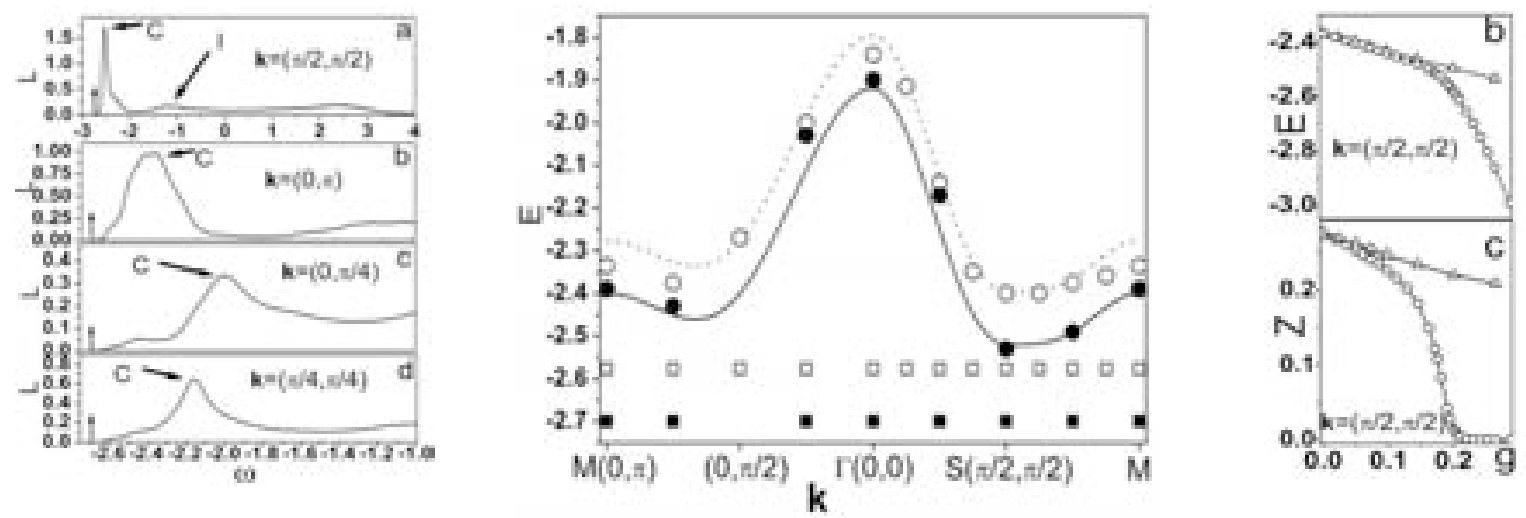

Figure 5.8: Left panel: Spectral function for the hole at different $k$ points for $J / t=0.3$ and $g=0.23$. Vertical arrows show the position of the zero-phonon line, and "C" marks the centroid of the $t-J$ band. Center panel: Dispersion of zero-phonon line (squares) and centroid (circles) for $g=0.23$ (filled) and $g=0.20$ (open) respectively. Lines are calculation for bare $t-J$ model. Right panel: Position of zero-phonon line (b) and zero-phonon line weight, $Z$, (c) as a function of coupling constant, $g$, using the diagrammatic Monte Carlo (circles) and phonon-phonon non-crossing approximation (triangles). Figures taken from A. Mishchenko and N. Nagaosa [141].

Monte Carlo results for the zero-phonon line and the incoherent centroid are shown as circles and squares, respectively, while the dispersion of the bare $t-J$ model is shown as lines. This demonstrates that even for dispersive electronic features, the intuitive result that the centroid of spectral weight tracks $\epsilon(\mathbf{k})$ even as a function of momentum holds true. In addition, the results from Mishchenko in Figure 5.8 show that in the quantum Monte Carlo calculations for this $t-J+$ phonon model, the reduction of $Z$ as a function of coupling constant, $g$ has a very rapid crossover to a small polaron state - this is unlike the simple IFB discussed earlier, where the zero-phonon line decreased smoothly as $e^{-g}$.

Later calculations by O. Rösch and O. Gunnarsson [170] confirmed the results from Mishchenko and Nagaosa, but used a slightly different approach. These calculations essentially dealt with systems that had no electron-phonon coupling in the initial state, i.e. insulators where no holes (electrons) were present in the valence (conduction) band in the initial state, and therefore this part of the Hamiltonian could be neglected for $\psi_{i}$. In this special case, they found that the photoemission intensity 


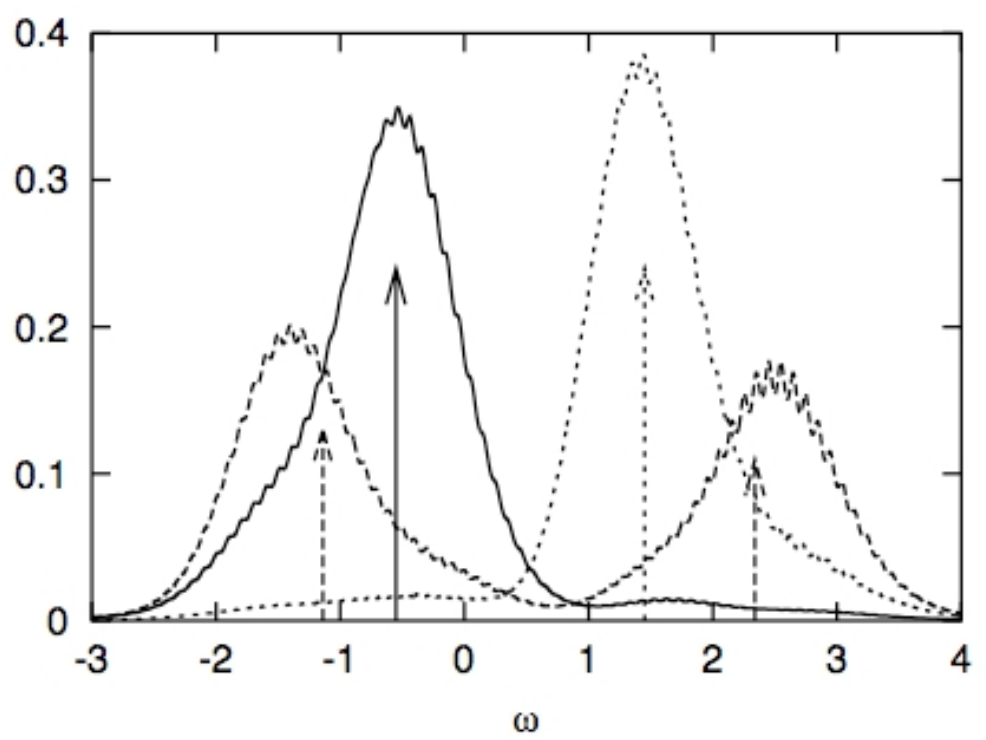

Figure 5.9: Spectral functions for creating a hole in the undoped 4-site Holstein-t- $J$ model $\left(t=1, J=0.3, \omega_{p h}=0.1, g=0.8\right) . k=0$ : solid line, $k= \pm \pi / 2$ : dashed line, $k=\pi$ : dotted line. Lorentzian broadening: $\mathrm{FWHM}=0.01$. Arrows show positions and weights of corresponding peaks for $g=0$. Figure taken from O. Rösch and O. Gunnarsson [170].

can then be simply expressed as an average over frozen distorted lattices without any electron-phonon interaction. This essentially removes any electron-phonon coupling term from the Hamiltonian, and reduces the problem to a sum over the different configurations of distortions. In this case, the spectral function of a single hole in both the Holstein and $t-J$ models were calculated, and it was found that the first moment of spectral weight coincided with the bare electronic dispersion, even as a function of momentum. Results from the Holstein model and $t-J$ model calculations are shown in Figures 5.9a and 5.9b, respectively.

These numerical studies of the single-electron spectral function in the presence of strong electron-phonon interactions demonstrate importantly that even in the case of small polaron formation, the majority of incoherent spectral weight can still exhibit strong momentum dependence and follow the original "frozen lattice" dispersion. However, this also indicates that simply observing dispersive spectral weight does not imply that the true eigenstates of the $N-1$ system are actually delocalized 
excitations. Therefore, in order to make such statements about the delocalization or mean free path of single-electron excitations using ARPES, more care must be applied to study the details of the lineshape in order to make statements about the nature of the actual eigenstates of the system.

\subsection{Momentum Dependence of Franck-Condon Broadening}

In addition to the dispersive centroid of incoherent weight, we have also found an intriguing momentum anisotropy of $\Gamma_{\text {LHB }}$ which may have profound implications for the emergence of the first doped hole states. In particular, our analysis reveals that as a function of momentum, the width of the LHB peak, $\Gamma_{\mathrm{LHB}}(\mathbf{k})$, increases directly proportionally to the binding energy of the peak maximum, $\varepsilon(\mathbf{k})$, such that $\varepsilon(\mathbf{k})=$ $\alpha \Gamma_{\text {LHB }}(\mathbf{k})$. Empirically, we have found that $\alpha=1.8$, and at $1.8 \Gamma$ away from the peak, the intensity has decayed to $\sim 10^{-4}$ of the peak value.

In Figure 5.10a, we show data from $\mathrm{Ca}_{2} \mathrm{CuO}_{2} \mathrm{Cl}_{2}$ together with Gaussian fits at various locations in $\mathbf{k}$-space, along with crosses to denote the position of $\varepsilon(\mathbf{k})+$ $1.8 \Gamma_{\mathrm{LHB}}(\mathbf{k})$. The momentum dependence of these features along both the $(0,0)-$ $(\pi, \pi)$ and $(0, \pi)-(\pi, 0)$ directions is summarized in Figures 5.10b and 5.10c. While the peak position changes considerably, the onset remains roughly fixed, and thus the width appears to change to accommodate the dispersion of the peak. In Figure 5.10a, we show data from $\mathrm{Ca}_{2} \mathrm{CuO}_{2} \mathrm{Cl}_{2}$ together with Gaussian fits at various locations in $\mathbf{k}$-space, along with crosses to denote the position of $\varepsilon(\mathbf{k})+1.8 \Gamma_{\mathrm{LHB}}(\mathbf{k})$. The momentum dependence of these features along both the $(0,0)-(\pi, \pi)$ and $(0, \pi)-(\pi, 0)$ directions is summarized in Figures 5.10b and 5.10c. While the peak position changes considerably, the onset remains roughly fixed, and thus the width appears to change to accommodate the dispersion of the peak.

In a simple picture where $\Gamma$ is related to the electron-phonon coupling strength (in

the independent boson model, $\left.\Gamma^{2} \propto g\right)$, the photohole would effectively be coupled more strongly to the lattice at $\mathbf{k}=(\pi, 0)$, the $d$-wave antinode, than at $\mathbf{k}=(\pi / 2, \pi / 2)$, 


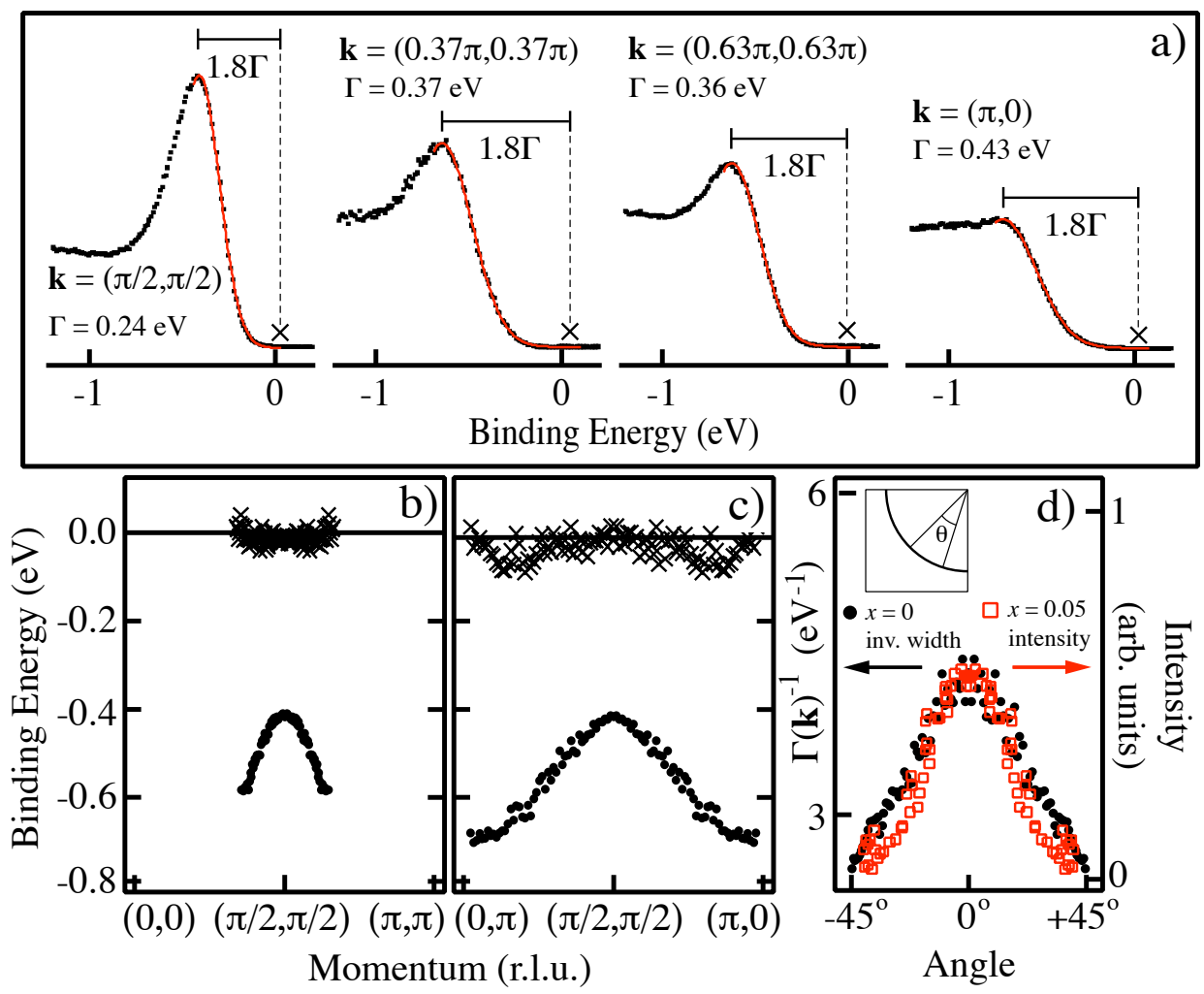

Figure 5.10: (a) Data from the LHB taken at various $\mathbf{k}$ points, together with Gaussian fits (red). k dependent dispersion of the LHB, $\varepsilon(\mathbf{k})$ (blue circles), along the $(0,0)-$ $(\pi, \pi)(\mathrm{b})$ and $(0,0)-(\pi, \pi)$ (c) directions. Crosses represent $\varepsilon(\mathbf{k})+1.8 \Gamma_{\mathrm{LHB}}(\mathbf{k})$. Data in (a)-(c) were taken at $200 \mathrm{~K}$. (d) Comparison of the low-energy spectral weight from $\mathrm{Ca}_{1.95} \mathrm{Na}_{0.05} \mathrm{CuO}_{2} \mathrm{Cl}_{2}$ with $\Gamma_{\mathrm{LHB}}(\mathbf{k})^{-1}$ as a function of angle, as defined in the inset.

the $d$-wave node. Recent results from $\mathrm{Ca}_{2-x} \mathrm{Na}_{x} \mathrm{CuO}_{2} \mathrm{Cl}_{2}$ have demonstrated that spectral weight first emerges near $(\pi / 2, \pi / 2)$ upon hole doping, while only faint intensity is visible near $(\pi, 0)$, and similar results were reported for other lightly hole doped cuprates such as $\mathrm{La}_{2-x} \mathrm{Sr}_{x} \mathrm{CuO}_{4}$ and $\mathrm{Bi}_{2} \mathrm{Sr}_{2} \mathrm{CaCu}_{2} \mathrm{O}_{8+\delta}[171,172]$. The momentum dependence of the inverse peak width in undoped $\mathrm{Ca}_{2} \mathrm{CuO}_{2} \mathrm{Cl}_{2}, \Gamma_{\text {LHB }}(\mathbf{k})^{-1}$, exhibits a qualitative similarity to the low-energy spectral weight in the lightly doped materials. This is shown in Figure 5.10d, where the spectral weight along the ostensible Fermi surface for $\mathrm{Ca}_{1.95} \mathrm{Na}_{0.05} \mathrm{CuO}_{2} \mathrm{Cl}_{2}$ is integrated in a narrow window around $\mathrm{E}_{\mathrm{F}}\left(\mathrm{E}_{\mathrm{F}} \pm 10 \mathrm{meV}\right)$ and compared with $\Gamma_{\mathrm{LHB}}(\mathbf{k})^{-1}$ along the antiferromagnetic zone 
boundary (dashed red) from the undoped system. The correspondence between these two quantities may suggest that the lack of well-defined QPs near $(\pi, 0)$ in the lightly doped compounds may be related to the apparently anisotropic coupling in the parent compound.

At low temperatures $(\mathrm{T}=200 \mathrm{~K})$, the Gaussian FWHM of the LHB is approximately $200 \mathrm{meV}$. In addition, the minimum separation between the Gaussian centroid and the pinned position of $\mu$ is approximately $350-400 \mathrm{meV}$. From the relation in Equation 5.1, if we take $\Delta P=350 \mathrm{meV}$ and $\Gamma=200 \mathrm{meV}$, we would obtain an $\omega_{0}=21 \mathrm{meV}$ and $g=17$. This is a surprisingly low frequency, considering that the majority of LO phonons are centered around $40 \mathrm{meV}$. If we assume that $\omega_{0}=$ $40 \mathrm{meV}$, we would expect $\Delta P=180 \mathrm{meV}$ and $g=4.5$. However, we have not yet observed any samples which have the peak centroid so close to $\mu$. On the other hand, all measurements were performed at elevated temperatures ( $\mathrm{T}>200 \mathrm{~K}$ ), so that the photohole can also absorb thermally excited phonons, as shown in Figure 4.10

It is also possible that the oversimplified independent boson model from which Equation 5.1 was obtained is insufficient to describe the physics of the polaron formation in the delocalized valence electrons. Using an independent boson model where momentum dependence is explicitly ignored to explain a strongly momentumdependent effect is obviously problematic.

On the other hand, very recent sophisticated calculations based on the exact diagrammatic quantum Monte Carlo techniques employed in [141] have been utilized to study the momentum dependence of the electron-phonon interaction in the undoped cuprates [173] (we have collaborated on this work, providing experimental spectra). These momentum-dependent results obtained from sophisticated exact diagrammatic calculations point towards a very similar behavior as observed experimentally, in that the Gaussian envelope becomes progressively broader towards $(\pi, 0)$ or $(0,0)$, proportional to the binding energy of the peak centroid, shown in Figure 5.11. These calculations are performed such that the actual electron-phonon coupling constant, $g$, as well the Einstein phonon dispersion, are both momentum-independent. Thus this effect arises entirely from the interaction between the dispersive electronic excitations and the dispersionless phonon field. 

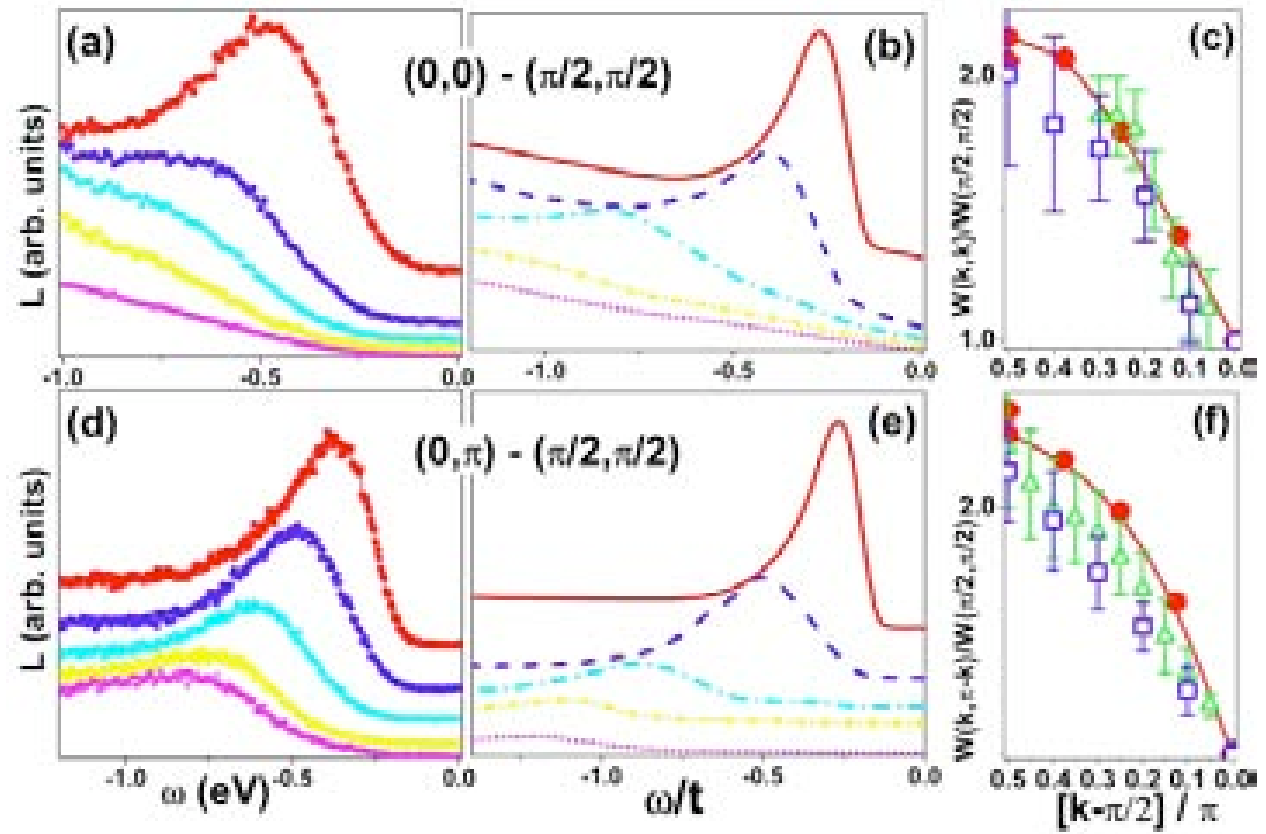

Figure 5.11: Experimental data along the $(0,0)-(\pi, \pi)$ direction (a), along with theoretical calculations for $\mathcal{A}(\mathbf{k}, \omega)$ (b). The relative width (normalized to $\Gamma(\pi / 2, \pi / 2)$ ) is shown in (c), with numerical values shown in red and results from $\mathrm{Ca}_{2} \mathrm{CuO}_{2} \mathrm{Cl}_{2}$ shown in green, and $\mathrm{Sr}_{2} \mathrm{CuO}_{2} \mathrm{Cl}_{2}$ in purple. The same plots along the $(\pi, 0)-(0, \pi)$ direction are shown in (d)-(f). Taken from [173].

\subsection{Polaron Formation and Hierarchy of Energy Scales}

Due to the strong coupling of the photohole to the lattice in the undoped cuprates, we may safely assume that the overlap of the single-electron excitations and the true eigenstates of the $N-1$ system is extremely small. One must then account for how the total spectral weight of the electron is distributed. First, for the case of the Mott insulator, one cannot satisfy sum rules for spectral weight using photoemission. This is simply because the spectral weight for one electron is divided into upper and lower Hubbard bands at all points in k-space (one can, in essence, think of the LHB as being purely spin-up, and the UHB as being spin-down, as opposed to a normal metal or band insulator with spin degeneracy). Therefore, particularly for the case of these 
a)

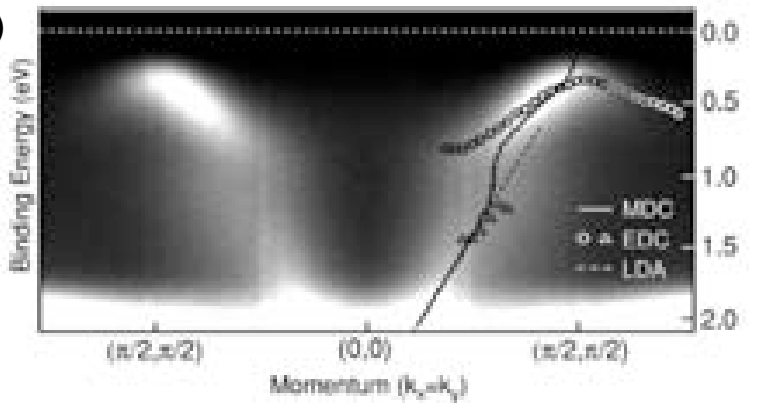

b)

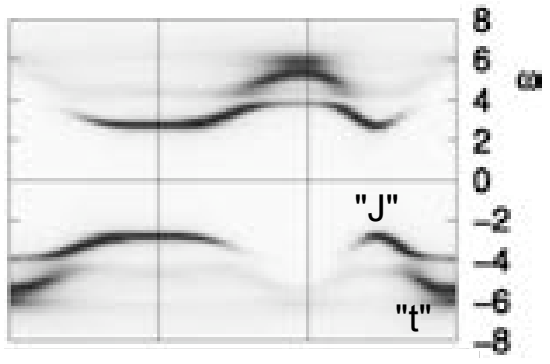

Figure 5.12: (a) Experimental data from $\mathrm{Ca}_{2} \mathrm{CuO}_{2} \mathrm{Cl}_{2}$ from [174] which show an ostensibly rapidly dispersing branch near $(0,0)$. Near $(\pi / 2, \pi / 2)$, the emission is dominated by the $J$ structure. (b) Numerical results from variational cluster perturbation theory [175] which demonstrate the same behavior of the $t$ and $J$ branches near $(0,0)$ (the rightmost part of the panel).

"strongly correlated systems", one must be careful to integrate over both occupied and unoccupied states before one can address any sum rules. Practically speaking, that means both direct and inverse photoemission must be performed.

In a idealized system of electrons coupled only to a vibrational mode, one can satisfy the conservation of total spectral weight by summing over all phonon sidebands, starting from 0-0. For instance, this should be the case for a system such as the $\mathrm{H}_{2}$ molecule, or perhaps the ionic alkali halides $(\mathrm{NaCl})$. In the undoped cuprates, however, there are multiple things to consider, such as the original band dispersion and the effects of electronic correlations and spin degrees of freedom. Therefore, the integrated intensity of the Gaussian LHB peak alone should not correspond to the total spectral weight associated with injecting a photohole, but there should be a manifold of higher energy excitations. This was actually observed in the calculations of Mishchenko in Figure 5.8 where there is substantial spectral weight spread out to high energies (the right of the figure). Earlier $t-J$ model calculations predicted that $Z \sim 0.2$, with $80 \%$ of the spectral weight pushed to higher energies due to strong electron correlation effects. This is also borne out in the calculations by Mishchenko, where feature "C", corresponding to the antiferromagnetic LHB (what we will also call the " $J$ " branch), encompasses about $20 \%$ of the spectral weight. Here, $80 \%$ of the spectral weight is retained at higher energies due to electron correlation effects. In 
fact, this high-energy incoherent spectral weight appears to follow a dispersion quite similar to the original, uncorrelated band (we call this the "t" branch). A number of numerical calculations based on a wide variety of approaches, including exact diagonalization [176], self-consistent Born approximation [158], quantum Monte Carlo [177], strong coupling perturbation theory [178], variational cluster perturbation theory [175], and cluster dynamical mean field theory [179] all appear to demonstrate the same general point: that a large amount of incoherent, high-energy spectral weight appears to possess a dispersion similar to the unrenormalized band.

A recent analysis of our ARPES measurements of $\mathrm{Ca}_{2} \mathrm{CuO}_{2} \mathrm{Cl}_{2}$ done by Filip Ronning [174] seems to provide some interesting experimental evidence along these lines. While at $(\pi / 2, \pi / 2)$, emission from the LHB or $J$ branch is dominant, near $(0,0)$ the situation is rather different. In particular, there is essentially no emission at the $(0,0)$ point, and this is not simply due to a matrix element selection rule, since no emission is seen in the second zone at $(2 \pi, 0)$ either. Instead, between $(\pi / 4, \pi / 4)$ and $(0,0)$, the spectral weight falls along a steeply dispersing band reminiscent of the unrenormalized band. Correspondingly, the numerical calculations cited above also seem to suggest a transfer of spectral weight from the $J$ branch to the $t$ branch upon reaching $(0,0)$. The calculations of Mishchenko suggest that the addition of phonons does not change the relative fraction of spectral weight in the $t$ and $J$ branches found in the earlier calculations which did not consider lattice relaxation effects. This is illustrated in Figure 5.12, where experimental data along the $(0,0)-(\pi, \pi)$ line are shown in Figure 5.12a, while one example of a calculation showing analogous behavior [175] is shown in Figure 5.12b. Although this is quite suggestive, it is well known that there are many states [131, 180], including possible "string resonances" [140], that exist at higher energies which could make positive identification of the $t$ branch rather difficult. Nevertheless, taken at face value, this provides a rather appealing hierarchy of excitations: an incoherent $t$ branch at high energies, an intermediate $J$ branch, and a small polaron state at the lowest binding energies. It is also rather intriguing that both the $t$ and $J$ structures exhibit significant dispersion, even though they are incoherent. However, given the work of Mishchenko and Rösch, allowing the incoherent branches to maintain the dispersion consistent with the underlying energy 

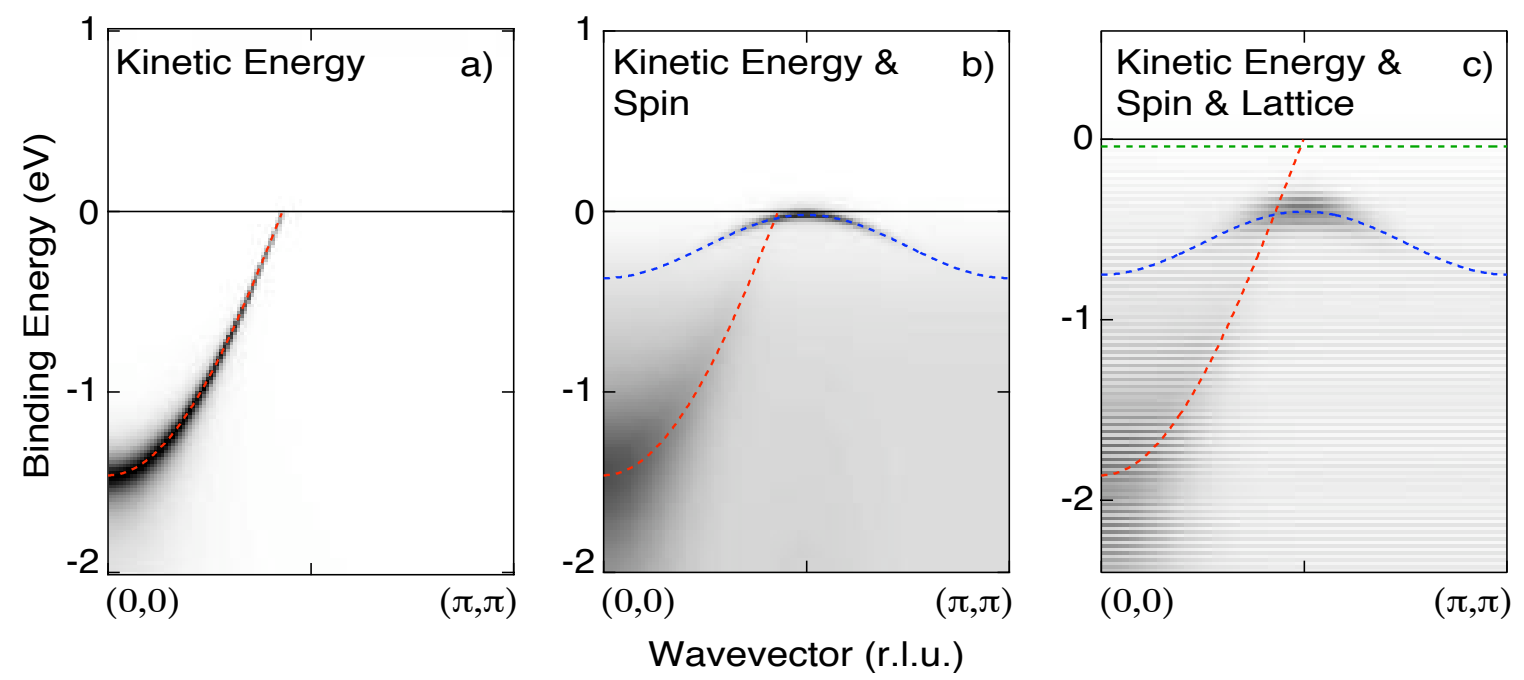

Figure 5.13: (a) ARPES spectra for a half-filled, Fermi-liquid-like band. (b) Effects of electron correlations and antiferromagnetism. (c) Cumulative effects of kinetic energy, electron-electron correlations and antiferromagnetism, and lattice effects on the ARPES spectra.

scales associated with those branches appears to be entirely reasonable. Therefore, we might attempt to physically visualize the $t$ branch as spectral weight associated with an electron hopping, but moving incoherently with respect to the antiferromagnetic background and the phonons. The $J$ branch would then be linked to the electron moving coherently within the antiferromagnetic background, but incoherently relative to the phonons. Finally the invisible QP (or small polaron state) is the real quasiparticle of the system, which moves coherently with respect to both the antiferromagnetic background as well as the phonons. Moreover, the spectral weight is distributed such that the $t$ branch encompasses $\sim 80 \%$ of the spectral weight, the $J$ branch $\sim 20 \%$, and the small polaron QP has $\rightarrow 0$.

In Figure 5.13, we show a schematic depiction of the effects of these three different energy scales on the ARPES spectra of the undoped cuprate $\mathrm{Ca}_{2} \mathrm{CuO}_{2} \mathrm{Cl}_{2}$. In the absence of electronic correlations, one would expect to see a broadly dispersing, sharp Fermi liquid-like band, shown in Figure 5.13a. Upon turning on electronic correlations, this single half-filled band would be split into a fully occupied LHB and an unoccupied UHB, each with a much narrower bandwidth, now corresponding to the 
magnetic exchange energy, $J$ (Figure 5.13b). Finally, lattice relaxation effects give rise to a Franck-Condon broadening of the spectral features in (b), as shown by the phonon sidebands in Figure 5.13c. This results in a broader distribution of spectral weight and the top of the LHB pushed out into the original Mott gap. This is also discussed in Figure 6.4 in Chapter 6. We should emphasize that in this heuristic picture, the kinetic energy and electron-electron repulsions are still critical elements and many of the main spectral features and the distribution of spectral weight are retained even in the presence of the FCB.

Finally, this picture only discusses lattice polaron formation as a potential source for the broadening of the LHB. In the absence of lattice effects, it is still unclear whether the spectral function of the LHB would possess sharp, $\delta$-function peaks (as predicted in the $t-J$ model) or be broadened due to electronic correlations. This is still very much an open and much-debated issue. Some theoretical work discussing possible broadening effects due to electronic correlations will be discussed below, with the exception of dynamical mean-field theory, which will be discussed in Chapter 6 . First, some analytical approximations have have suggested that interactions with the antiferromagnetic spin background cause $Z \rightarrow 0[181,182]$. On the other hand, recent work by Stanescu and Phillips [183, 184, 185] has attempted to tackle the 2D Hubbard model by starting with the Hubbard $U$ (as opposed to $t-J$ models) and using a selfconsistent two-site dynamical cluster expansion. This approach is similar in some respects to cluster dynamical mean-field theory (CDMFT). Work using this approach also finds that coherent, QP-like excitations do not exist in the Mott insulator [184], consistent with the redistribution of the electron spectral weight into a UHB and LHB. Although these results are rather intriguing, the calculations by Stanescu and Phillips show that the excitations in the LHB are sharp and well-defined at $(0,0)$, while they are much broader at $(\pi / 2, \pi / 2)$, directly opposite to what is observed experimentally. Thus, while the approach taken by Phillips and Stanescu could be qualitatively correct, the existing calculations do not appear to accurately reproduce the quantitative features in the experimental data. 


\section{Chapter 6}

\section{Doping Dependence of the Electronic Structure}

Understanding the doping dependence of the cuprate superconductors away from the parent Mott insulating state has been, perhaps, the key intellectual issue in the study of high-temperature superconductivity ${ }^{1}$. Apart from the high transition temperatures themselves, the defining characteristic of the cuprates has been the fact that at half-filling, these materials are Mott insulators, a product of strong electron-electron repulsions. Despite this fact, ARPES studies of the evolution of the cuprates away from the Mott insulator have been lacking, mainly due to a lack of suitable materials for such studies. For instance, $\mathrm{Bi}_{2} \mathrm{Sr}_{2} \mathrm{CaCu}_{2} \mathrm{O}_{8+\delta}$, the canonical cuprate superconductor studied by ARPES, cannot typically be grown at low hole doping levels due to the constraints of crystal chemistry. For this reason, $\mathrm{Ca}_{2-x} \mathrm{Na}_{x} \mathrm{CuO}_{2} \mathrm{Cl}_{2}$ is nearly unique in its suitability for the study of the evolution of the Mott insulator to the high- $\mathrm{T}_{\mathrm{c}}$ superconductor. From a materials perspective, the only other potential candidate to span the lightly doped cuprate phase diagram is $\mathrm{La}_{2-x} \mathrm{Sr}_{x} \mathrm{CuO}_{4}$; however, the potential difficulties with $\mathrm{La}_{2-x} \mathrm{Sr}_{x} \mathrm{CuO}_{4}$ are that the lower Hubbard band features have never been identified clearly in the undoped compound, and also that certain spectral features in $\mathrm{La}_{2-x} \mathrm{Sr}_{x} \mathrm{CuO}_{4}$ have been associated with phase separation. Therefore, it is

\footnotetext{
${ }^{1} \mathrm{~A}$ large portion of the work discussed in this chapter has been published in K.M. Shen et al., Physical Review Letters 93, 267002 (2004).
} 
not entirely clear how representative $\mathrm{La}_{2-x} \mathrm{Sr}_{x} \mathrm{CuO}_{4}$ would be of the high- $\mathrm{T}_{c}$ cuprates on the whole. For this reason, a detailed understanding of the doping evolution of the cuprates from the perspective of $\mathrm{Ca}_{2-x} \mathrm{Na}_{x} \mathrm{CuO}_{2} \mathrm{Cl}_{2}$ would be extremely valuable, and is the topic of this chapter.

One of the central topics addressed by theory in the doping evolution of the cuprate superconductors is the evolution of the chemical potential, $\mu$. As mentioned in Chapter 1, this particular topic was the subject of intense debate over the past decade due to conflicting reports over the whether the chemical potential remained pinned inside the Mott gap with doping, or whether it shifted inside the lower Hubbard band. Consequently, this would in principle allow one to determine whether the doped low-energy electronic states formed as mid-gap states, or whether they evolved continuously from the LHB states. It turns out that much of this confusion stemmed from where the true "top" of the lower Hubbard band was located, as we have discussed in Chapters 4 and 5. The discussion of the doping evolution of the electronic structure in this chapter will be based on a synthesis of different experimental measurements. First and foremost is the experimental determination of the chemical potential, $\mu$. To do this, we develop a new method to determine with high precision the shift in $\mu$ as a function of hole doping. We combine this quantitative determination of $\mu$ with high-resolution ARPES measurements of the low-lying states along the $(0,0)-(\pi, \pi)$ nodal direction. We find that only by using the Franck-Condon broadening (FCB) scenario introduced in Chapters 4 and 5 can we explain this doping evolution in a self-consistent fashion. By integrating all these different aspects, we find that we can explain the doping evolution of $\mathrm{Ca}_{2-x} \mathrm{Na}_{x} \mathrm{CuO}_{2} \mathrm{Cl}_{2}$ as a simultaneous shift of $\mu$, combined with an increase of the quasiparticle residue, $Z$, away from zero.

However, the most important element of this doping evolution is the utilization of the polaronic / FCB phenomenology. In particular, even if $\mu$ has been determined in an accurate fashion, a critical problem remains if the true nature of the LHB has not been properly identified. For instance, previous interpretations of the LHB pointed to the maximum of spectral weight as the "top" of the LHB, whereas in the (what we now believe to be correct) polaronic scenario, the position of the QP pole for the LHB is within the low-energy tail of spectral weight. In the previous interpretation, 
$\mu$ would have been shifting continuously within the Mott gap, which would have been posed a blatant logical inconsistency. However, our new identification of the position of the QP pole resolves this potentially fatal flaw.

\subsection{Determination of the Chemical Potential Shift}

In many prior studies of the chemical potential shift in other cuprates, primarily by the Fujimori group, the shift of various core level states was used to estimate the shift in the chemical potential, $\Delta \mu$. However, this interpretation is somewhat nontrivial due to a number of factors. First, the intrinsic width of the short-lived core hole states $(\sim 1-2 \mathrm{eV})$ makes an accurate determination of the core level shift (to say, better than $50 \mathrm{meV}$ ) rather difficult. Secondly, the core level shift, $\triangle C L$ is known to be a combination of numerous factors

$$
\Delta C L=-\Delta \mu+K \Delta Q+\Delta V_{M}+\Delta E_{R}
$$

where $K \Delta Q$ is the chemical shift, $\Delta V_{M}$ is the change in the Madelung potential, and $\Delta E_{R}$ is the change in the core hole screening [53]. Therefore, any reasonable estimates of $\Delta \mu$ must be able to accurately account for all additional factors. Being able to explain the individual shifts of all the different core levels is therefore a rather tricky task, and the precision of this approach is further limited by the broad core hole lineshapes. Finally, the lack of an x-ray source and the limited energy range of the monochromator $(14-32 \mathrm{eV})$ at our beamline meant that even attempting to measure the core levels was impossible and therefore finding an alternative method for determining the chemical potential shift was essential.

For these studies, we chose to utilize particular states in the shallow valence band as reference states to determine $\Delta \mu$ as a function of hole doping. In particular, we chose to use oxygen $2 p$ states at particular k-points which were identified in Chapter 4. As discussed earlier, previous band structure calculations showed these states to have zero overlap with the wavefunction of the LHB (assumed to be of Zhang-Rice singlet character). Because these states were fairly low in energy $(<-4 \mathrm{eV})$, we 

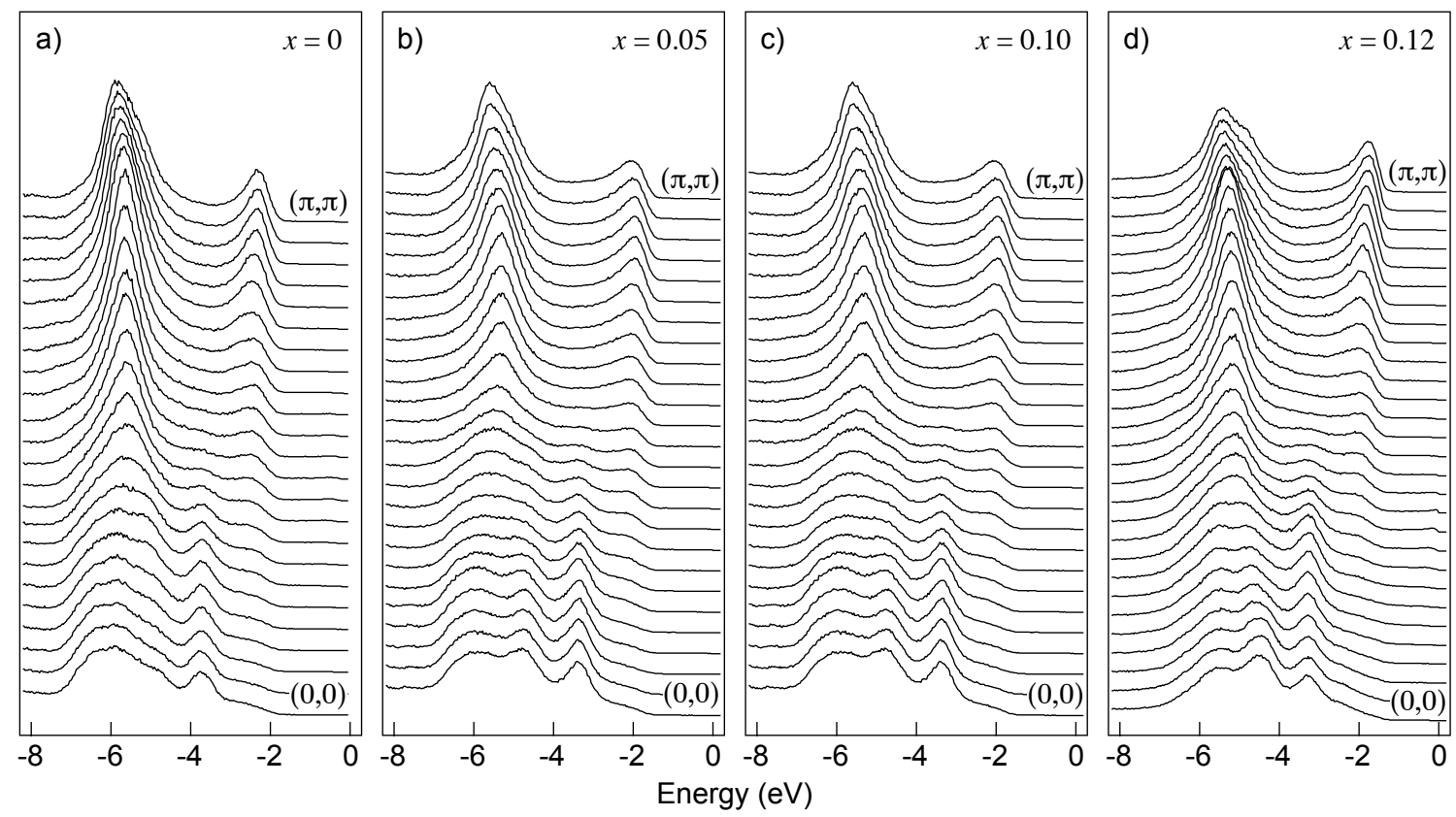

Figure 6.1: (a)-(d) Valence band EDC stacks taken from $(0,0)$ to $(\pi, \pi)$ at $h \nu=25.5$ $\mathrm{eV}$ and polarization at $45^{\circ}$ to the $\mathrm{Cu}-\mathrm{O}$ bond direction for $x=0,0.05,0.10$, and 0.12 samples, respectively.

also assumed that the relative contributions from changes in the Madelung potential or the screening energy would be considerably less than those of deep core levels (although possibly not negligible). In addition, the intrinsic linewidth of these shallow valence band states was easily less than $1 \mathrm{eV}$, so the determination of the positions of these valence band states could be accomplished with a relatively high degree of precision. This was a key aspect of our studies of the chemical potential shift in $\mathrm{Ca}_{2-x} \mathrm{Na}_{x} \mathrm{CuO}_{2} \mathrm{Cl}_{2}$. EDC stacks of valence band spectra for $x=0,0.05,0.10$, and 0.12 are shown in Figure 6.1.

On a practical level, the $\mathrm{O} 2 p_{\pi}$ and $\mathrm{O} 2 p_{z}$ states are good reference states to track because they are particularly pronounced, unlike many other electronic states in the valence band. In particular, the $\mathrm{O} 2 p_{\pi}$ state has been identified in a number of doped cuprates, and is almost solitary at $\sim 2 \mathrm{eV}$ binding energy at $(\pi, \pi)$. This fact makes the $\mathrm{O} 2 p_{\pi}$ state uniquely suited for tracking the doping dependent shift of $\mu$. The O2p state, on the other hand, sits within the manifold of other states in the valence band, 


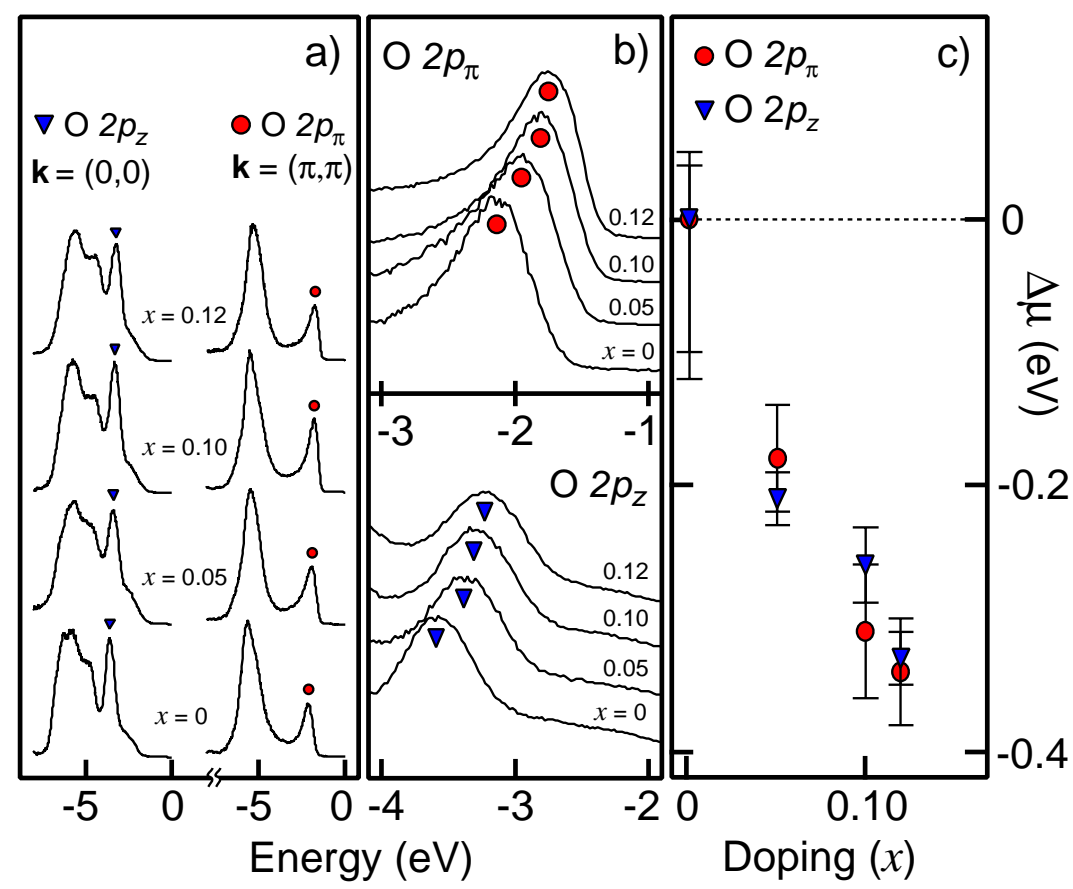

Figure 6.2: (a) Valence band spectra taken at $h \nu=25.5 \mathrm{eV}$ and polarization at $45^{\circ}$ to the $\mathrm{Cu}-\mathrm{O}$ bond direction for $x=0,0.05,0.10$, and 0.12 samples at $\mathbf{k}=(0,0)$ and $(\pi, \pi)$, respectively. (b) An expanded plot of the shift for the $\mathrm{O} 2 p_{z}$ peak at $(0,0)$ (triangle) and the $\mathrm{O} 2 p_{\pi}$ peak at $(\pi, \pi)$ (circle). (c) The doping dependent shift of the $\mathrm{O} 2 p_{\pi}$ and $\mathrm{O} 2 p_{z}$ peaks summarized from multiple samples. Data are plotted relative to $\mu_{0}$ determined from the lower bound of the pinned chemical potentials in the undoped insulator.

but at an excitation energy of $h \nu=25.5 \mathrm{eV}$, the $\mathrm{O} 2 p_{z}$ peak is quite pronounced and can its position can be extracted quite reliably. Because both states are constituted from orbitals coming from the $\mathrm{CuO}_{2}$ plane, these states could in principle be utilized for all cuprates, not just NaCCOC. Furthermore, as discussed in Chapter 4 , the O2 $p_{\pi}$ and $\mathrm{O} 2 p_{z}$ states are also not hybridized with the ZRS state and can be interpreted in a rather simple fashion for these studies.

Valence band spectra from $\mathrm{Ca}_{2-x} \mathrm{Na}_{x} \mathrm{CuO}_{2} \mathrm{Cl}_{2}$ taken at $h \nu=25.5 \mathrm{eV}$ and the incident polarization at $45^{\circ}$ to the $\mathrm{Cu}-\mathrm{O}$ bond direction are shown in Figure 6.2a. The individual $\mathrm{O} 2 p_{\pi}$ and $\mathrm{O} 2 p_{z}$ states are denoted by circles and triangles, respectively. In Figure 6.2b, we show an expanded plot of the shift of these peaks as a function 
of doping, illustrating that these peaks can indeed be used as a sensitive metric for $\Delta \mu$. These measurements were repeated for a number of samples at each doping level, to ensure a high level confidence in $\Delta \mu$. In addition, the accurate determination of $\mu_{0}$ in Chapter 4, the lowest pinned position of $\mu$ in the insulator, or in other words, the highest possible energy for the "hidden" quasiparticle in the insulator, was also critical. Without this determination, it would be futile to discuss any change in $\mu$ in going from $x=0$ to $x=0.05$. However, we can now use our determined value of $\mu_{0}$ as an effective upper bound for the top of the LHB in the undoped Mott insulator. The combination of this first determination of $\mu_{0}$ along with our measurement of $d \mu / d n$ allows us state unequivocally that the $\mu$ appears to shift continuously above the position of the LHB peak centroid - i.e. within the supposed Mott gap. However, if we utilize the polaronic / FCB model proposed for the undoped insulator and extend it to the case of the doped compounds, we can easily resolve this apparent logical inconsistency. The doping dependent shift of $\mu$ is shown in Figure 6.2c where the shifts from the $\mathrm{O} 2 p_{\pi}$ and $\mathrm{O} 2 p_{z}$ valence band states are shown. By taking a weighted average of the $\mathrm{O} 2 p_{\pi}$ and $\mathrm{O} 2 p_{z}$ states, our values for $\Delta \mu$ are as follows (referenced from $\left.\mu_{0}=0\right): \Delta \mu_{0.05}=-0.20 \mathrm{eV} ; \Delta \mu_{0.10}=-0.28 \mathrm{eV} ; \Delta \mu_{0.12}=-0.33 \mathrm{eV}$, with typical error bars of $\pm 0.025 \mathrm{eV}$. However, the relative shift $\Delta \mu=-0.20$ from $x=0 \rightarrow 0.05$ has the largest amount of error, because of the inherent difficulty in determining $\mu_{0}$ accurately.

In addition, we compare our results from valence band measurements to those performed from core level XPS measurements of polycrystalline samples, taken from Yagi et al. [186] (squares). In Figure 6.3, we combine our valence band measurements with XPS measurements from a wide range of samples $\left(\mathrm{La}_{2-x} \mathrm{Sr}_{x} \mathrm{CuO}_{4}, \mathrm{Bi}_{2} \mathrm{Sr}_{2} \mathrm{CaCu}_{2} \mathrm{O}_{8+\delta}\right.$, $\left.\mathrm{Bi}_{2} \mathrm{Sr}_{2} \mathrm{CuO}_{6+\delta}\right)$ taken from the Fujimori group's measurements [53, 55]. However, for the XPS measurements of $\mathrm{Ca}_{2-x} \mathrm{Na}_{x} \mathrm{CuO}_{2} \mathrm{Cl}_{2}$, there was no accurate determination of $\mu_{0}$, so the absolute position of these points on this plot is arbitrary, and can be shifted up or down. Despite the obvious difference in the approaches of the valence band and core level shift analyses, we find good agreement between the two measurements for $\mathrm{Ca}_{2-x} \mathrm{Na}_{x} \mathrm{CuO}_{2} \mathrm{Cl}_{2}$. If we estimate a value for $d \mu / d n$, taken from the $x=0.05,0.10$, 


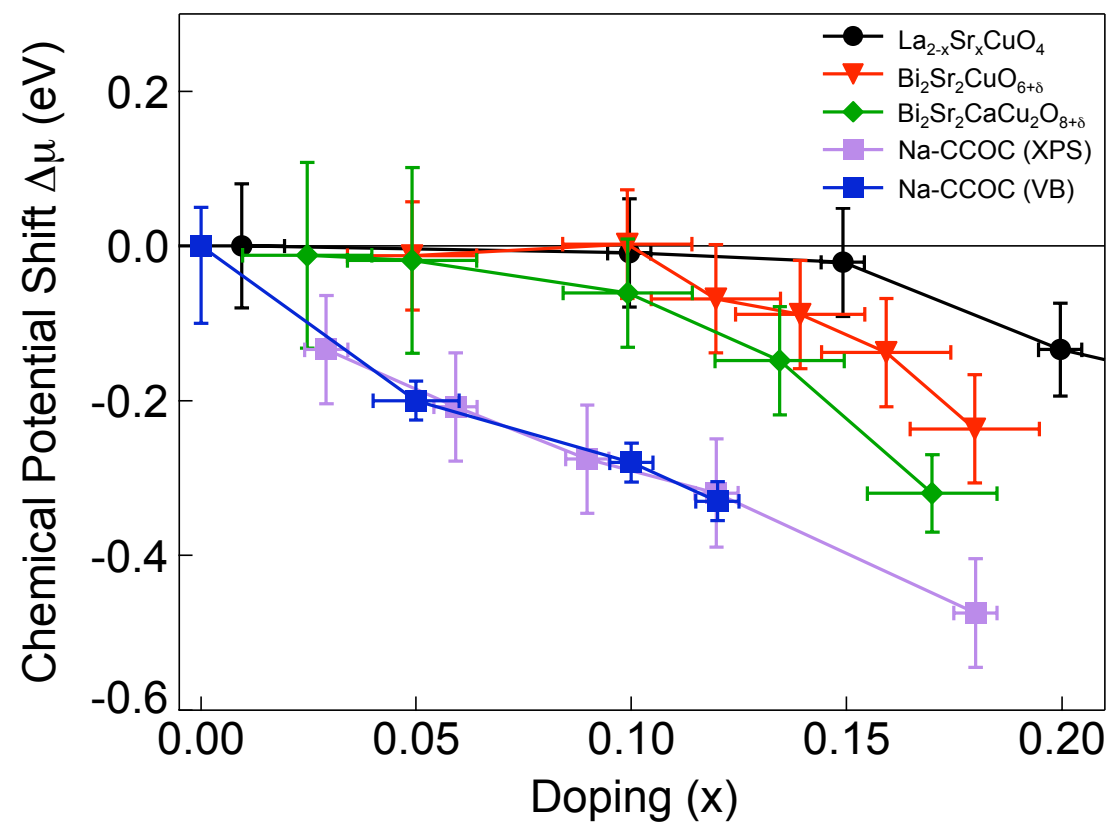

Figure 6.3: Chemical potential shift determined from photoemission spectroscopy of $\mathrm{La}_{2-x} \mathrm{Sr}_{x} \mathrm{CuO}_{4}, \mathrm{Bi}_{2} \mathrm{Sr}_{2} \mathrm{CaCu}_{2} \mathrm{O}_{8+\delta}, \mathrm{Bi}_{2} \mathrm{Sr}_{2} \mathrm{CuO}_{6+\delta}$, and $\mathrm{Ca}_{2-x} \mathrm{Na}_{x} \mathrm{CuO}_{2} \mathrm{Cl}_{2}$. All measurements are determined by core level spectroscopy from the Fujimori group [55], except Na-CCOC (VB), which was determined from our own ARPES measurements.

and 0.12 samples (neglecting the $x=0$ composition, due to the larger error bars associated with $\mu_{0}$ ), we obtain $d \mu / d n=-1.8 \pm 0.5 \mathrm{eV} /$ hole. This is roughly on par with, although even somewhat larger than the estimates from band structure calculations which give $\sim-1.3 \mathrm{eV} /$ hole [124]. The estimates from XPS give a $d \mu / d n \sim 2.2 \pm 0.8$ $\mathrm{eV} /$ hole; the rate of the doping dependent shifts from valence band and core level measurements appear to coincide reasonably well, demonstrating the compressibility of the $\mathrm{Ca}_{2-x} \mathrm{Na}_{x} \mathrm{CuO}_{2} \mathrm{Cl}_{2}$ system. However, there is an obvious discrepancy between results obtained on $\mathrm{Ca}_{2-x} \mathrm{Na}_{x} \mathrm{CuO}_{2} \mathrm{Cl}_{2}$ and $\mathrm{La}_{2-x} \mathrm{Sr}_{x} \mathrm{CuO}_{4}$, with the results from the Bi-based cuprates somewhat intermediate between $\mathrm{Ca}_{2-x} \mathrm{Na}_{x} \mathrm{CuO}_{2} \mathrm{Cl}_{2}$ than $\mathrm{La}_{2-x} \mathrm{Sr}_{x} \mathrm{CuO}_{4}$.

This observation of $d \mu / d n \sim 0$ [53] for lightly doped $\mathrm{La}_{2-x} \mathrm{Sr}_{x} \mathrm{CuO}_{4}$ suggested that there were two qualitatively different scenarios for the doping evolution of the cuprates. Some studies suggested that there either was a "chemical potential shift", 
akin to a simple rigid band shift that would be observed in heavily doping a band insulator. Earlier evidence for this was obtained from observations of the valence band shift in $\mathrm{Bi}_{2} \mathrm{Sr}_{2} \mathrm{CaCu}_{2} \mathrm{O}_{8+\delta}$ [54], core level shifts in $\mathrm{Bi}_{2} \mathrm{Sr}_{2} \mathrm{CaCu}_{2} \mathrm{O}_{8+\delta}$ [55], and a combination of resonant photoemission and x-ray absorption for $\mathrm{La}_{2-x} \mathrm{Sr}_{x} \mathrm{CuO}_{4}$ and $\mathrm{Nd}_{2-x} \mathrm{Ce}_{x} \mathrm{CuO}_{4}$ [56]. On the other hand, other studies seemed to suggest that $\mu$ was pinned in mid-gap upon hole doping [53, 52]. This would have suggested two fundamentally different physical pictures for the doping evolution of the cuprates. However, much of this confusion stemmed from the misidentification of the top of the LHB, where (up to this work) it was thought that the peak maximum at $(\pi / 2, \pi / 2)$ was the pole at the top of the LHB. Utilizing the polaronic / FCB model allows us to explain many features of the doping evolution of $\mathrm{La}_{2-x} \mathrm{Sr}_{x} \mathrm{CuO}_{4}$ and $\mathrm{Ca}_{2-x} \mathrm{Na}_{x} \mathrm{CuO}_{2} \mathrm{Cl}_{2}$ within a common framework, as the low-energy QPs would still form at the top of the LHB in both materials. However, this does not explain the quantitative differences in the behavior of $\mu$ in across these two compounds.

\subsection{Doping Evolution of the Low Energy States}

However, the most important aspect of this accurate quantification of $\mu$ is that this allows to directly compare between the low-energy spectra of different compositions, with a clear idea of where $\mu$ resides in one composition relative to another. This is shown in Figure 6.6, where EDCs taken along the $(0,0)-(\pi, \pi)$ nodal direction are shown at all four different doping levels. In these plots, the data are plotted on an absolute scale relative to $\mu_{0}$, such that $\mathrm{E}_{\mathrm{F}}$ for each doping level is shifted by $\Delta \mu$ for that concentration, relative to $\mu_{0}$. By combining the values determined for $\Delta \mu$, we can clearly observe a number of striking features in the spectra. Most importantly, as already discussed, is the fact that $\mu$ does not immediately drop to the position of the peak of the LHB in the undoped parent insulator. In fact, it was this initial observation that inspired the adoption of the polaronic / Franck Condon broadening approach outlined throughout this thesis. Instead, in going from the $x=0$ compound to $x=0.05$, it appears that the position of the broad hump maximum remains roughly fixed relative to $\mu_{0}$, and that the effect of hole doping is simply to "eat" into the 
a)
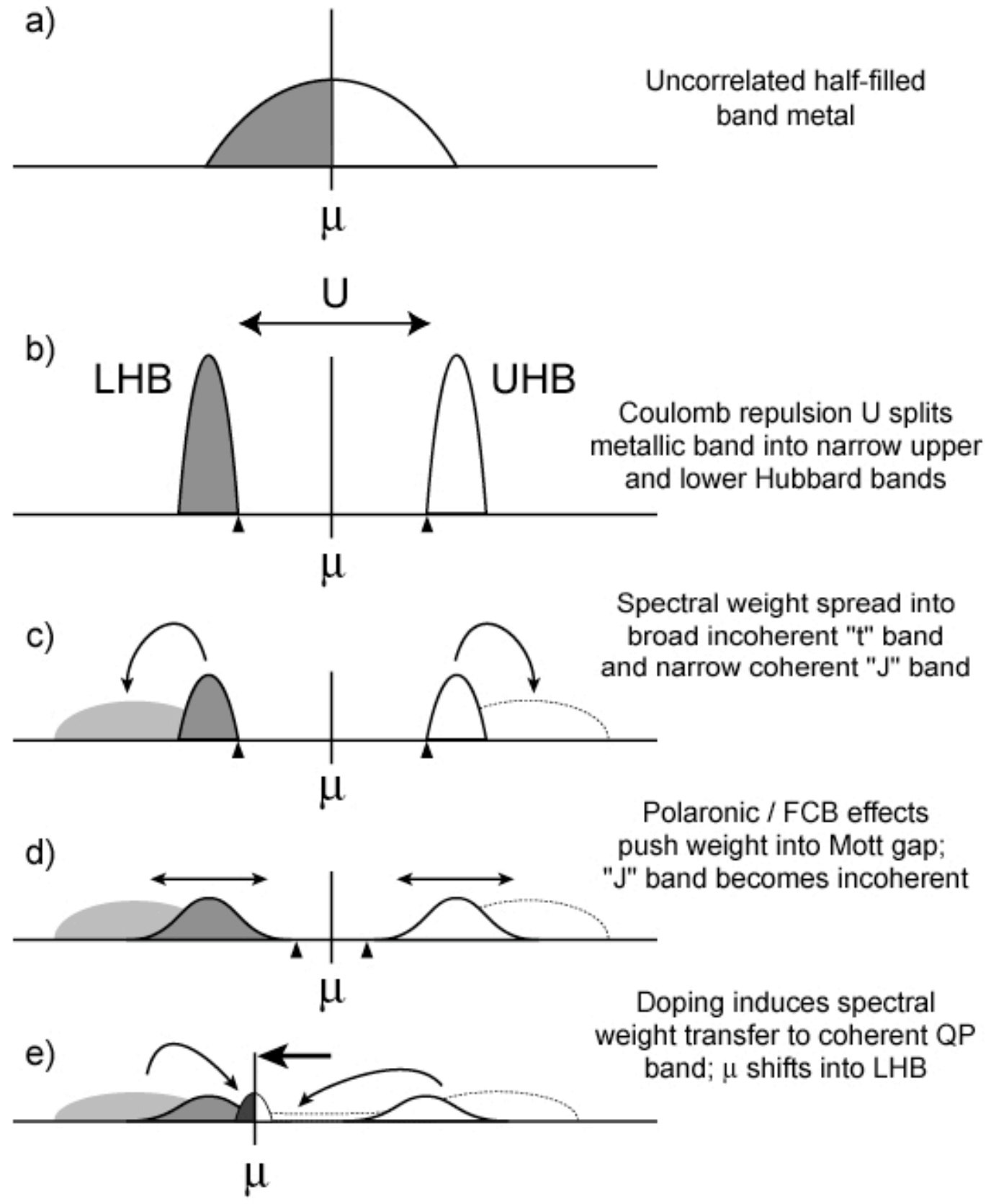

Figure 6.4: Proposed model for the density of states in the undoped cuprates. A detailed explanation of this model is explained in the text. 
intensity of the undoped LHB feature. Clearly, if the LHB is "structureless", that is, consisting of only a single pole, this type of doping evolution would make no sense. In general, the position of this broad hump maximum shifts with hole doping by nearly the same amount as $\mu$. In addition to this shift, low-energy spectral weight develops at $\mathrm{E}_{\mathrm{F}}$ and forms into a well-defined peak for $x=0.10$ and 0.12 compositions.

In Figure 6.4, we give a comprehensive overview of the doping and "interaction" evolution of the density-of-states within our model. Figures 6.4(a)-(d) discuss the undoped cuprate, while (e) shows the evolution with hole doping. In (a), we take the simplest situation of the undoped cuprates without electron-electron correlations, so that the material is a half-filled metal with $\mathrm{a} \sim 3 \mathrm{eV}$ bandwidth. Strong electronelectron interactions split this single metallic band into a completely occupied lower Hubbard band and an unoccupied upper Hubbard band, shown in (b). The energy gap between the LHB and the UHB corresponds to the onsite Coulomb repulsion between two electrons on the same site, $U$. In this simplified scenario, we neglect the fact that these materials are charge-transfer insulators (with an energy gap, $\Delta$ ) and use a single-site picture. In (b), we take the simple view that the LHB and UHB are like the valence and conduction band in a band insulator, such that all spectral weight is essentially coherent. Numerical calculations show that strong electronic correlations push $\sim 80 \%$ of the spectral weight into an incoherent high-energy feature, discussed in Chapter 5, giving the situation in (c). Because this incoherent high-energy weight may have a dispersion similar to the original unrenormalized band, we call this the " $t$ " band. This leaves only $\sim 20 \%$ of the spectral weight in the coherent low-energy " $J$ " band. However, polaronic effects cause FCB in the $t$ and $J$ bands, shown in (d), so that the $J$ band is now composed of incoherent multi-phonon satellites. This also has the effect of reducing the size of the single-particle gap. The position of the top of the LHB and bottom of the UHB are denoted by the triangles. In (b) and (c), the gap magnitude is given by $U$. However, lattice relaxation pushes spectral weight into this Mott gap, such that in (d), the true QP gap (or polaron gap) is substantially reduced from $U$. Finally, when we dope holes into the system, the chemical potential shifts to the top of the LHB. This doping also induces a spectral weight transfer to a band of coherent QPs which disperses across $\mathrm{E}_{\mathrm{F}}$ and whose intensity scales with $x$. 


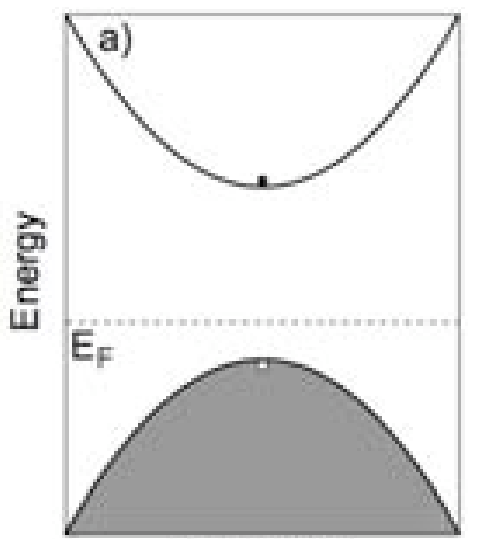

Momentum

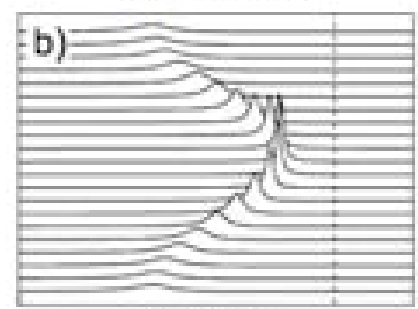

Energy

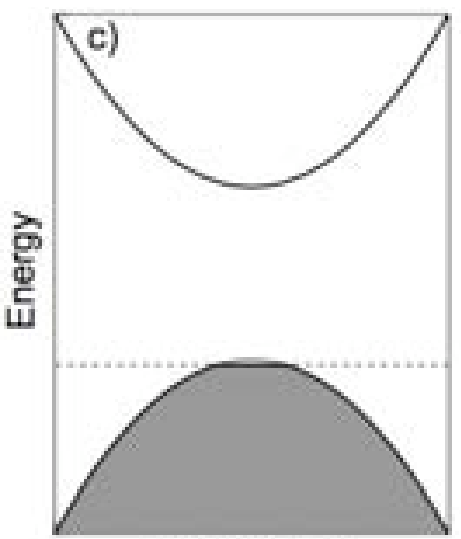

Momentum

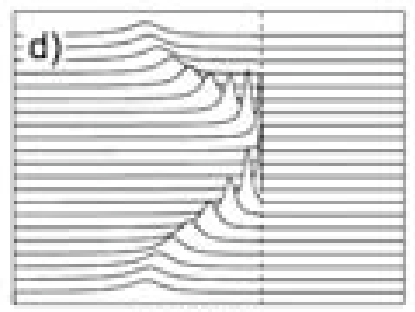

Energy

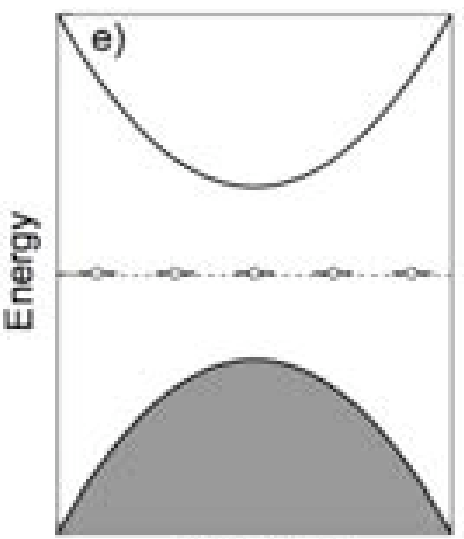

Momentum

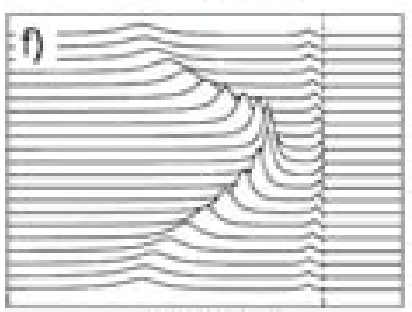

Energy

Figure 6.5: (a) In a pure insulator, the chemical potential at $\mathrm{T}=0$ is undefined, since there is a finite energy difference (the gap energy) between adding one electron and removing one electron. Typically, $\mathrm{E}_{\mathrm{F}}$ is pinned by impurity states in the gap. (b) Rigid band shift into valence band, corresponding to fairly heavy doping of a band insulator. (c) In-gap states, consistent with a lightly doped semiconductor.

The model outlined in Figure 6.4 draws upon three key aspects. First, FCB redefines where the "top" of the LHB lies, so that $\mu$ does shift to the top of the LHB with doping, and does not sit in mid-gap. Secondly, the chemical potential shifts continuously within the LHB as a function of doping, with a rate determined by the emergent QP band. Third, spectral weight is continuously transferred to this QP band with hole doping at a rate roughly proportional to $x$. We can easily contrast this with the conventional band insulator / semiconductor scenarios, shown in Figure 6.5. In this case, there is no FCB for the band insulator, and the excitations at the top of the VB are sharp, $\delta$-function-like peaks (in fact, Figure 6.5a is incorrect in a rigorous sense, since there should be no electron-hole decay at all energies below the gap energy, so even the higher binding energy peaks should also be $\delta$-function-like). 


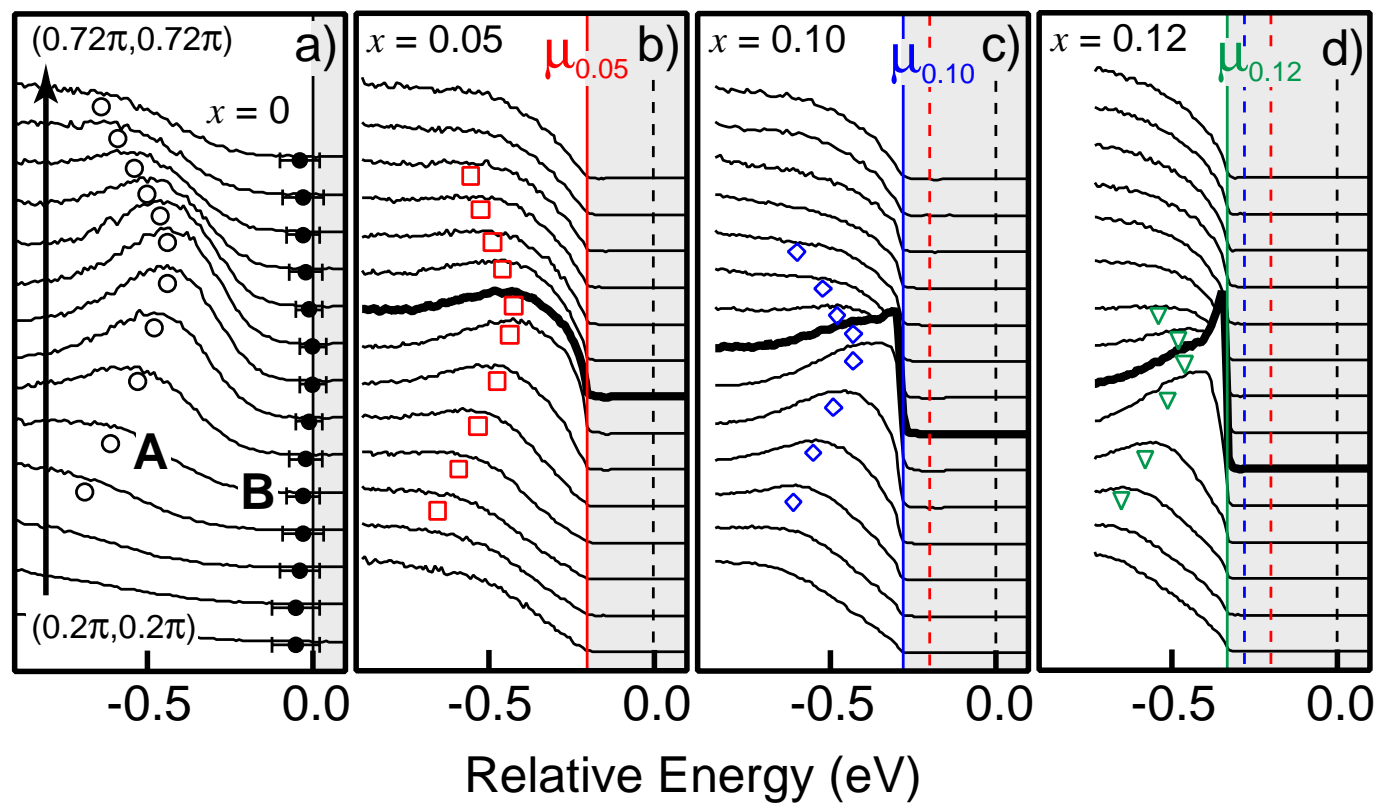

Figure 6.6: Doping dependence of EDCs along the nodal direction from $(0.2 \pi, 0.2 \pi)$ - $(0.72 \pi, 0.72 \pi)$ for $x=0$ (a), 0.05 (b), 0.10 (c), and 0.12 (d). Data were taken with $h \nu=25.5 \mathrm{eV}$ and polarization at $45^{\circ}$, at $15 \mathrm{~K}$ for the doped samples and $200 \mathrm{~K}$ for the undoped sample. Hump maxima are determined from a combination of fitting and second derivatives. Data are plotted on an absolute scale relative to $\mu_{0}$, but $\mathrm{E}_{\mathrm{F}}$ for each individual sample is marked above.

A shifting chemical potential would imply the scenario in Figure 6.5b, where the material becomes metallic (or a semi-metallic) and the single electron excitations at $\mathrm{E}_{\mathrm{F}}$ are again $\delta$-functions. A pinned chemical potential, on the other hand, would imply Figure 6.5c, where the spectral features of the band insulator are essentially retained, and a small, narrow band is formed in mid-gap from impurity states. The data we shown in Figure 6.6 obviously deviates dramatically from any of the scenarios shown in Figure 6.5, and demonstrates the importance of the physical picture proposed in Figure 6.4.

The global picture for the doping evolution of $\mathrm{Ca}_{2-x} \mathrm{Na}_{x} \mathrm{CuO}_{2} \mathrm{Cl}_{2}$ is best summarized in Figures 6.7a and 6.7b. In Figure 6.7a, we clearly illustrate the momentum and doping dependence of the both the broad high-energy hump and the low-energy quasiparticle bands together in a single plot. Plotted on an energy scale relative to $\mu_{0}$, 
it becomes evident that the energy position of the hump maximum does not change appreciably with doping, although at higher doping levels, particularly $x=0.12$, accurately identifying the position of the hump maximum becomes difficult. Nonetheless, from the overall trend, this appears to be a robust statement at least at low doping levels. At low energies, we track the position of the low energy spectral weight using an MDC analysis. By tracking the low-energy dispersion across doping levels, we reveal rather striking and unexpected behavior: the low energy quasiparticle dispersion lies approximately on a single straight line, with only relatively small changes in the group velocity as a function of doping, consistent with the "universal nodal velocity" discussed by Zhou et al. [187]. Moreover, the convergence of the group velocity, $v_{\mathrm{F}}$, the change in nodal wavevector, $\mathbf{k}_{\mathrm{F}}$, and the chemical potential shift, $\Delta \mu$, neatly merge together in such a way that it appears that $\mu$ is simply rigidly shifting down the band defined by the low-lying quasiparticles, while effectively ignoring the broad hump at higher energies. Experimentally, $v_{\mathrm{F}} \sim 1.8 \mathrm{eV} \cdot \AA$, and the values for the nodal $\mathbf{k}_{\mathrm{F}}$ were determined (from a series of Fermi surface mappings and long "arc-to-arc" cuts) to be $\mathbf{k}_{\mathrm{F}}=0.50 \pm 0.02,0.47 \pm 0.02,0.43 \pm 0.01$, and $0.41 \pm 0.01$, for $x=0$, $0.05,0.10$, and 0.12 , respectively. From these values, we basically obtain that $\Delta \mathbf{k}_{\mathrm{F}}$ $\sim \Delta \mu / v_{\mathrm{F}}$, directly tying $\mu$ to $\mathbf{k}_{\mathrm{F}}$, as would be for the case of doping a simple Fermi liquid system.

\subsection{Theoretical Interpretations of the Doping Evolution}

\subsubsection{Doping Dependence of Electron-Boson Coupling}

While this aforementioned aspect is somewhat reminiscent of hole doping a conventional band insulator, the fact that $Z \rightarrow 0$ in the undoped system is highly unlike the conventional Fermi liquid case. Therefore, this aspect of transfer from incoherent to coherent spectral weight is truly a hallmark of strong correlation effects. This is illustrated in Figure 6.7b, where EDCs at $\mathbf{k}_{\mathrm{F}}$ along the $(0,0)-(\pi, \pi)$ line are plotted, along with a superimposed caricature of the proposed distribution of incoherent (pink) and 

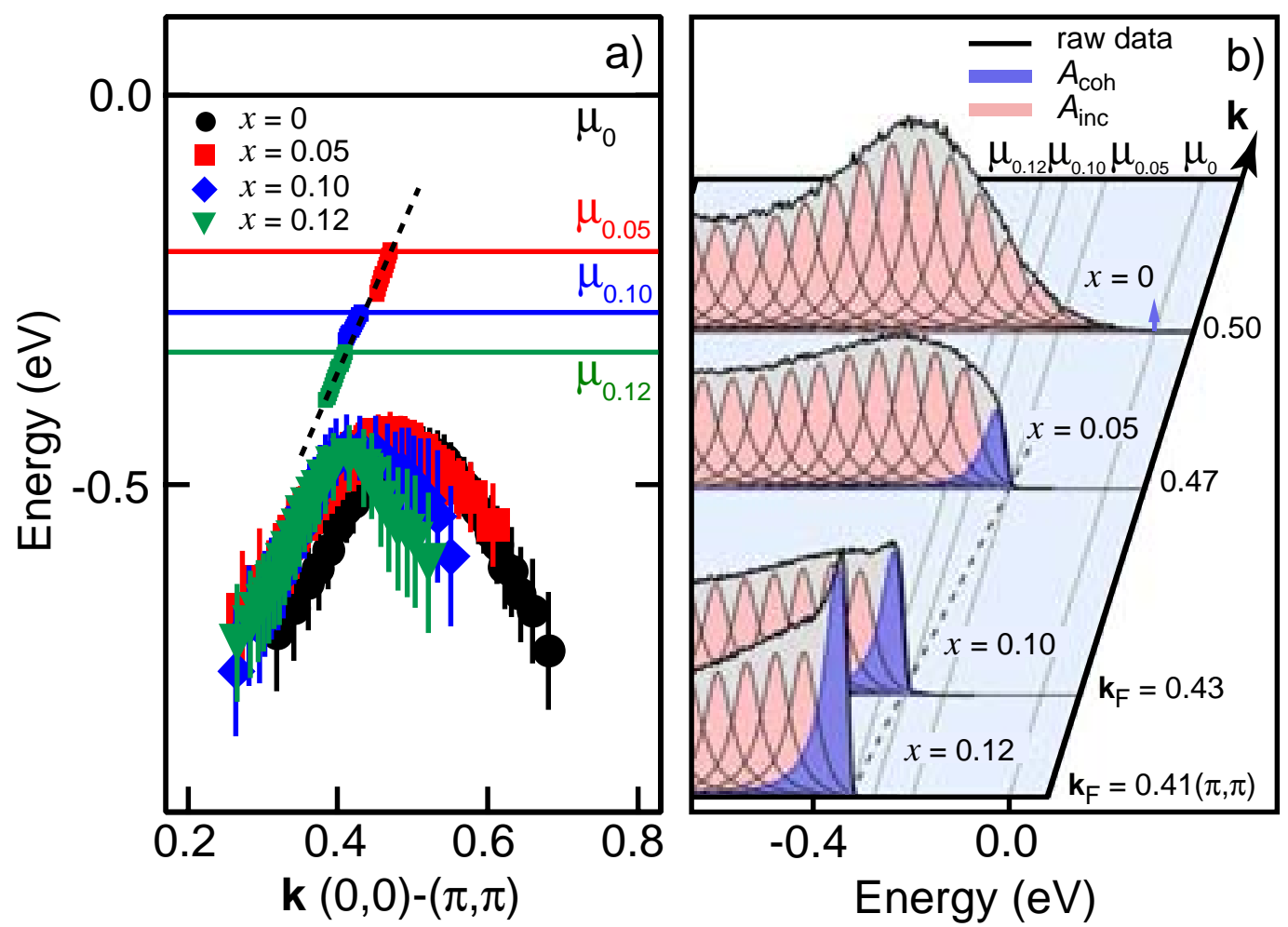

Figure 6.7: (a) Summary of the doping dependence of the broad hump (in symbols, also shown in Figure 6.6) and the low-energy MDC dispersions. All data are plotted relative to $\mu_{0}$, although $\mathrm{E}_{\mathrm{F}}$ for each composition is denoted above. (b) Doping dependence of the nodal EDC at $\mathbf{k}_{\mathrm{F}}$, combined with a cartoon schematic of the proposed distribution of coherent (blue) and incoherent (pink) spectral weight, and its evolution as a function of doping.

coherent (blue) spectral weight. Doping then induces a transfer from incoherent to coherent weight, consistent with the growth of the low-energy peak. In the polaronic / FCB scenarios, the transfer from incoherent (boson satellites) to coherent ('0-0' transition) can be achieved simply by reducing the strength of the electron-boson coupling constant. As one approaches the Mott insulating state, the strength of the electronphonon coupling should increase rather dramatically as the screening from the mobile conduction electrons (holes) vanishes, ultimately leading to the formation of lattice polarons in the undoped system, as previously discussed. It is also worthwhile to note that the effective "strength" of the coupling of the photohole to the antiferromagnetic 
background should also increase towards half-filling. Although a detailed discussion of this is beyond the scope of this thesis, it is worth noting that the mechanisms for electron-phonon and electron-magnon coupling are markedly different. While the electron-phonon coupling is simply the electrostatic Coulomb interaction between the mobile electrons and the nuclei, the electron-magnon coupling in the Mott insulator is more complicated. Because it is simply the hopping of the hole in the Mott insulator that induces spin-flip processes, the "electron-magnon interaction" is really just a re-expression of strong electron-electron interactions. This is an obvious statement in this case, because it is the low-energy electrons of interest that also carry the spin- $1 / 2$ antiferromagnetism.

The conventional wisdom regarding metal-insulator transitions describes two general phenomenologies: "filling control" and "bandwidth control" metal-insulator transitions [188]. In the filling control case, the number of free carriers are gradually reduced to zero as the insulator is approached, but the effective mass, $m^{*}$, remains constant as this transition is crossed. On the other hand, for the bandwidth control transition, the number of carriers is kept constant, while $m^{*}$ diverges as the insulator is reached. Using this somewhat crude description for these two cases, we can actually see that the Mott insulator to high- $\mathrm{T}_{\mathrm{c}}$ superconductor evolution in $\mathrm{Ca}_{2-x} \mathrm{Na}_{x} \mathrm{CuO}_{2} \mathrm{Cl}_{2}$ borrows from both aspects of the bandwidth and filling control metal-insulator transitions. The fact that $v_{\mathrm{F}}$ remains finite suggests that $m^{*}$ also remains finite (this is a rather simplistic view; in reality, $v_{\mathrm{F}}$ does decrease somewhat at low dopings, but there is no evidence for $v_{\mathrm{F}} / v_{\text {band }}$ diverging), and $m^{*} / m_{\text {band }}$ can be seen as roughly proportional to the quasiparticle residue, $Z$, since the formal definition for $Z=(1-d \operatorname{Re} \Sigma / d \omega)^{-1}$, which is essentially the change of slope in the quasiparticle dispersion away from the unrenormalized band dispersion. The evolution to the superconducting state is obviously driven by hole doping (filling control), and in the context of $v_{\mathrm{F}} / v_{\text {band }}, m^{*}$ certainly remains finite. However, we have also established that $Z \rightarrow 0$ as one approaches the Mott insulator, suggesting that $m^{*}$ does diverge at the Mott insulating state. In fact, it is more meaningful to describe the evolution from the Mott insulator to the superconductor more precisely as one where the quasiparticle spectral weight, Z, evolves from near zero, but where the 
group velocity of the quasiparticles remains fixed. This then presents the rather confusing situation of making $m^{*} / m_{\text {band }}$ either finite or infinite, depending precisely on whether $m^{*}$ is defined relative to the spectral weight or the group velocity, and one must use a precise description of how determines the quasiparticle renormalization.

The coupling of the holes to bosonic fields (likely phonons) are responsible for a large degree of the broadening observed in the undoped insulator, and a reduction of this coupling strength with hole doping seems to be a plausible explanation for the subsequent changes in electronic structure. Along these lines, Mishchenko et al. have expanded on their initial work [141] on the single hole in the extended $t-J$ model + phonons by attempting to simulate the physics of hole doping by changing the coupling constant [173]. Specifically, they have performed their exact diagrammatic Monte Carlo technique on an extended $t-J$ model with higher-order hopping terms, $t^{\prime}$ and $t^{\prime \prime}$, since it is generally found that the $t-t^{\prime}-t^{\prime \prime}-J$ models do a better job of approximating the experimental data than the bare $t-J$ model; their calculations of the extended $t-J$ model give essentially the same basic physics as their earlier $t-J$ model calculations. However, the interesting question to investigate is whether one can reproduce what is observed in experiment by simply reducing the electronphonon coupling constant in these $t-t^{\prime}-t^{\prime \prime}-J+$ phonon models. At a generic level, the calculation appears to reproduce certain key aspects of the doping dependence of $\mathrm{Ca}_{2-x} \mathrm{Na}_{x} \mathrm{CuO}_{2} \mathrm{Cl}_{2}$. Certainly, the broad linewidth and exact spectral lineshape of undoped $\mathrm{Ca}_{2} \mathrm{CuO}_{2} \mathrm{Cl}_{2}$ is explained beautifully by the $t-t^{\prime}-t^{\prime \prime}-J+$ phonon calculations, as was described in Chapter 5. The famous "peak-dip-hump" and dispersion "kink" observed in many cuprates and taken to be signatures of electron-boson coupling are also reproduced by these calculations; however, this should not be surprising, since these types of structures are generic features of spectral functions with relatively strong electron-boson interactions. A more remarkable aspect of these calculations was the ability of these calculations to reproduce quite closely the momentum dependence of the width observed in the undoped compound discussed in Chapter 5. 


\subsubsection{Possible Relationship to Electron-Electron Correlations}

Another critical question regarding the doping dependence is whether electron-boson coupling alone can explain all the essential physics. Quite clearly, the hole doping should not only affect the magnitude of the electron-phonon coupling, but also the long-range antiferromagnetic order as well as the effective electron-electron correlations. From the aforementioned discussion, we have demonstrated that certain salient features in the carrier doping evolution of $\mathrm{Ca}_{2-x} \mathrm{Na}_{x} \mathrm{CuO}_{2} \mathrm{Cl}_{2}$ seem to be explained nicely within an electron-phonon framework. Here we will discuss other theoretical scenarios which neglect electron-phonon interactions, but simply focus on the effects of electronic correlations. In regards to most theoretical studies of the undoped insulator, the model of choice has been the $t-J$ model. In many respects, the $t-J$ model over-exaggerates the effects of electron-electron correlations, since doubly-occupied states are Gutzwiller-projected out of the Hilbert space. Therefore, the $t-J$ model is a situation where $U \rightarrow \infty$, and thus does not adequately treat the effects of charge fluctuations when $U$ is finite, as is the case for real materials. To properly treat the dynamic effects of double occupancy, a better approximation is the Hubbard model where $U$ is finite and double occupancy is permitted (the $t-J$ model can be thought effectively as the $U \rightarrow \infty$ limit of the more general Hubbard model). However, allowing doubly occupied states makes numerical solutions of the Hubbard model much more costly and difficult, since a greater degree of realism will always come at some appreciable cost.

One of the most successful recent theoretical approaches to treating the problem of double occupancy and charge fluctuations in a sophisticated manner is that of $d y$ namical mean field theory (DMFT) and its close variants, such as cluster dynamical mean field theory (CDMFT) $[189,190]$. Because a great deal of work in DMFT has centered precisely on the Mott insulator-to-metal transition, DMFT and its relevance to the data described here warrants some discussion. In a nutshell, the concept of DMFT is to replace a lattice problem with many degrees of freedom by a much simpler effective single-site problem coupled to a self-consistent bath. In spirit, it is similar to an Anderson impurity problem - a single atomic site embedded in some medium. Because the complex many-body dynamics are replaced by a a self-consistent bath, 

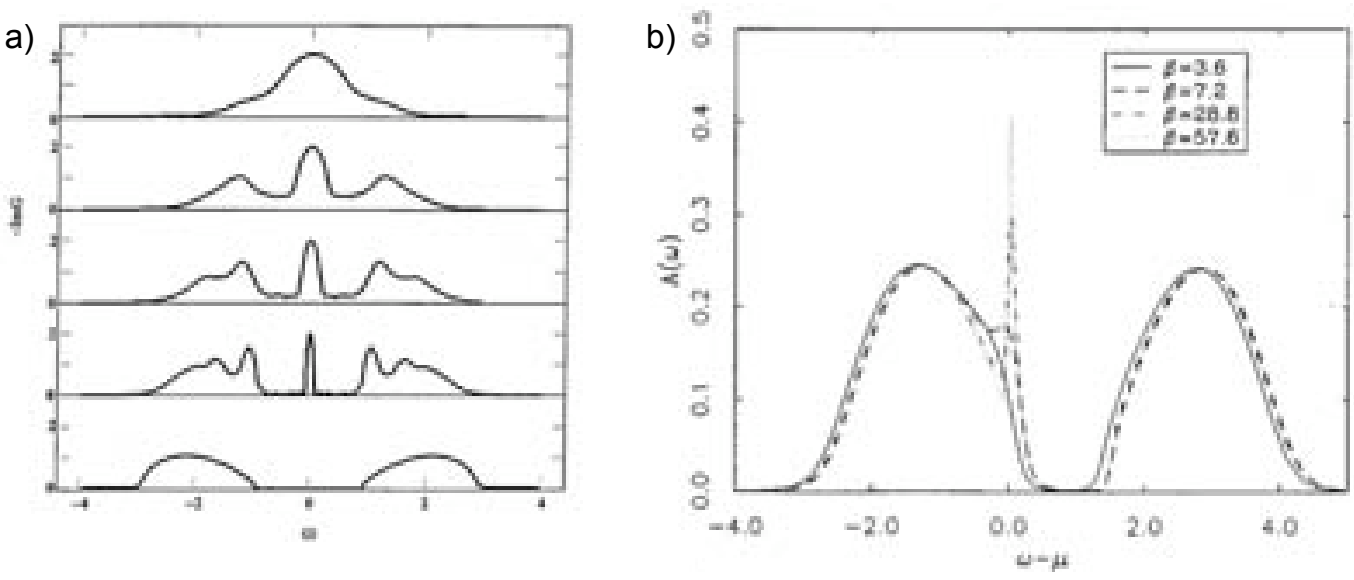

Figure 6.8: (a) Results from the Mott insulator-to-metal transition from DMFT as a function of $U / D$ [189]. (b) Similar results away from half-filling showing the QP peak at the top of the LHB, also from DMFT [191].

this is a mean-field approach, and DMFT attempts to interpolate between two limits: the localized atomic site (the single site, decoupled from the bath) and the delocalized band electrons (the self-consistent medium, without any impurity sites). Moreover, because this is a mean-field approach, one should expect DMFT to produce better results when the coordination of nearest neighbor sites (in the real problem) is large, thereby reducing the effects of large spatial fluctuations in the field. Here we discuss some of the results from DMFT describing the Mott insulator-to-metal transition. One approach is to study this transition as a progressive function of Coulomb repulsion; this is shown in Figure 6.8 where the density of states (DOS) is plotted at different ratios of the Hubbard $U$ over the half-bandwidth, $D: U / D$. As can be seen in Figure 6.8a, the DMFT calculations appear to nicely reproduce a major feature observed in experiment: in the Mott insulator, one has broad, incoherent Hubbard bands, and as one reaches the metallic phase, a narrow, coherent quasiparticle resonance appears with a quasiparticle residue, $Z$, which grows from zero [189]. This occurs not as a function of hole doping, but as a function of relative correlation strength, $U / D$. At different values of $U$ and $D$, one can have this resonance form not in the middle of the Mott gap, but at the top of the lower Hubbard band, as shown in Figure 6.8b [191]. 
Although the plots in Figure 6.8 are very redolent of the experimental situation shown in Figure 6.7, we should also make clear the important distinctions between the DMFT calculations and our experimental results. First, all work in the conventional DMFT approach is performed using a single site, meaning that their obtained $\mathcal{A}(\mathbf{k}, \omega)$ is momentum-integrated. Hence, at a practical level, the results obtained by DMFT should be compared with a DOS obtained by PES, and not single EDCs obtained in ARPES. Therefore, the resemblance between the EDCs in Figure 6.7 and the calculations shown in Figure 6.8 should not be taken literally, particularly because the experimental spectra are so strongly momentum dependent, as will be discussed in this chapter. In addition, MDC-derived dispersions at low doping levels appear to indicate fairly dispersive QPs. The narrow QP resonances obtained by DMFT would seem to indicate flat, non-dispersive bands, in contrast to experiment. These problems in relating ARPES spectra to DMFT might be addressed by very recent work on CDMFT, which considers not a single-site, but rather a small cluster of sites (such as a square of 4 sites) embedded within a self-consistent medium. This would then allow one to address both the momentum dependence of the self-energy together with charge fluctuations in a self-consistent manner.

At this point, the logical justification for using the Franck-Condon broadening model over DMFT to explain the features in the lightly doped $\mathrm{Ca}_{2-x} \mathrm{Na}_{x} \mathrm{CuO}_{2} \mathrm{Cl}_{2}$ are due to a number of reasons. First of all, the FCB scenario explains the pinned position of $\mu$ in the insulator, as well as the evolution of $\mu$ with doping in a very natural manner. It is also unclear whether DMFT could reproduce the key features of the doping evolution of $\mu$. Secondly, the fact that the Gaussian lineshape observed in the insulator is very easily explained within the context of the FCB model would also seem to suggest that polaron formation / Franck-Condon broadening would be the natural way to understand the spectral features of the insulator. This line of reasoning is further supported by the success of the $t-t^{\prime}-t^{\prime \prime}-J+$ phonon calculations of the spectral function from Mishchenko. However, this does not necessarily exclude the fact that DMFT calculations could possibly produce similar results. Along these lines, comparisons of the detailed single-particle lineshape obtained from future CDMFT work and ARPES would be extremely interesting. In particular, if more 
advanced CDMFT calculations could reproduce the observed Gaussian broadening in the momentum-resolved lineshapes seen in ARPES, the correct width, as well as its momentum dependence, then this would pose a serious challenge to the $t-t^{\prime}-t^{\prime \prime}-J$ + phonon models. However, one piece of evidence which would suggest that lattice polaron formation and not electron-electron repulsion is the driving force for the broad spectral lineshapes observed in the lightly doped cuprates is the very broad Gaussian lineshape observed for the $\mathrm{O} 2 p_{\pi}$ state at $(\pi, \pi)$, as discussed in Chapter 5. For the $\mathrm{O} 2 p_{\pi}$ state, the electronic correlation effects addressed by DMFT should be mostly irrelevant, but the $\mathrm{O} 2 p_{\pi}$ peak possesses a spectral lineshape very similar to that observed in the lower Hubbard band. However, DMFT may also capture much of the essential physics of the lightly doped cuprates, and one might speculate that a combination of DMFT + phonons may potentially provide the best model for describing the doping evolution of the cuprates.

\subsection{Doping Dependence of Franck-Condon Broadening for $\mathrm{O} 2 p_{\pi}$}

Because the lineshape of the $\mathrm{O} 2 p_{\pi}$ state played an important role in our determination that the observed FCB was primarily a result of lattice polaron formation, as shown in Chapter 5 , we now discuss the evolution of the $\mathrm{O} 2 p_{\pi}$ lineshape as a function of hole doping in detail. From simple considerations, one might expect a number of things to occur as the hole concentration is increased. First, one might expect the lineshape to change from Gaussian to more Lorentzian-like (Voigt-shaped) as the scattering rate of the photohole with other conduction electrons may increase due to the higher carrier concentration. Secondly, one might expect the Gaussian linewidth to decrease as the effective electron-phonon interaction is expected to be reduced at higher doping levels. Using the change in the LHB lineshape to track the doping dependence of the ostensible electron-boson coupling is difficult, since the spectral weight becomes cut off at $\mu$. Furthermore, there may also be doping dependent effects which are unrelated to the change in the electron-phonon coupling strength, and more in line with strong 

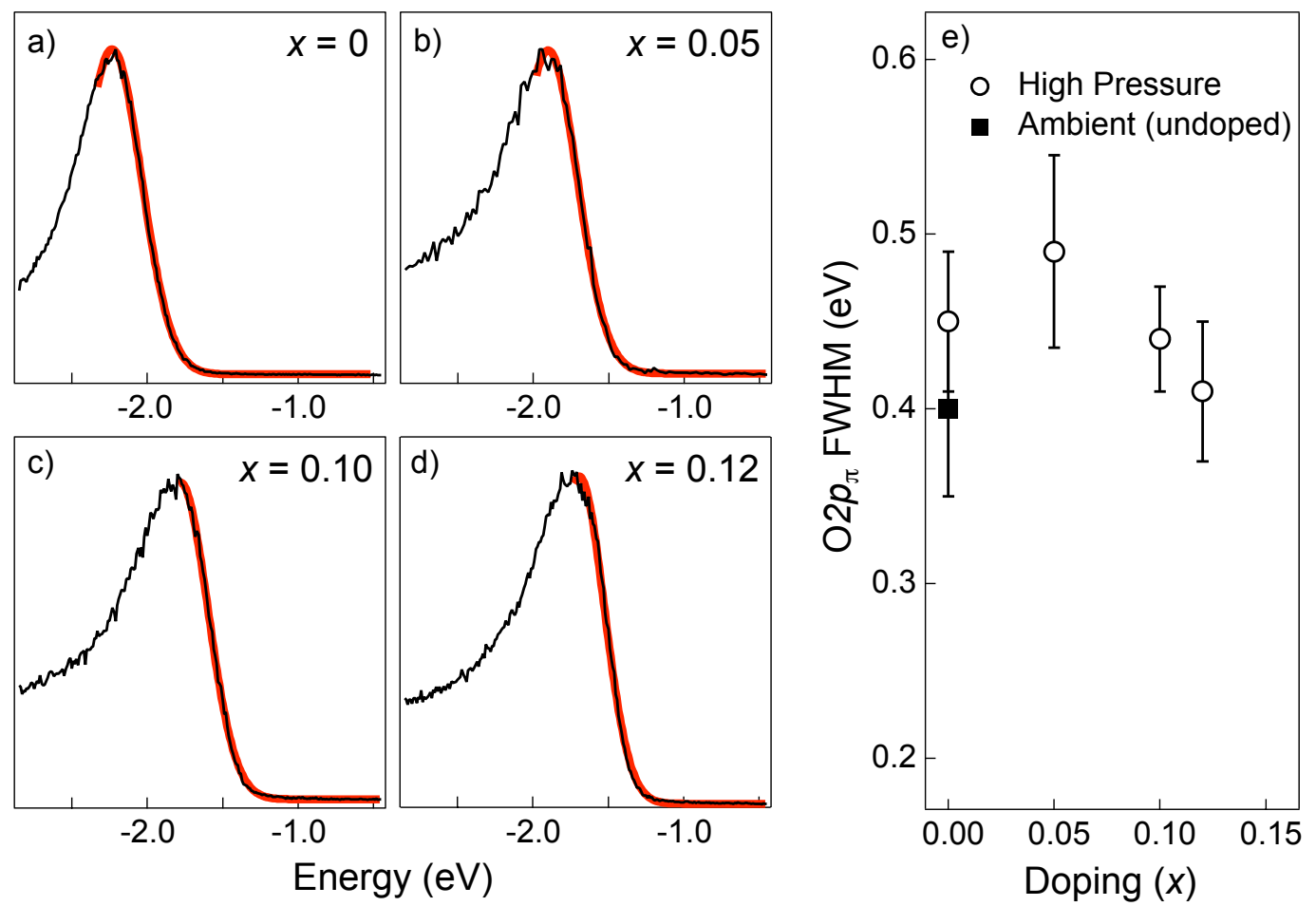

Figure 6.9: (a)-(d) $\mathrm{O} 2 p_{\pi}$ peak at $\mathbf{k}=(\pi, \pi)$ taken with $h \nu=25.5 \mathrm{eV}$ and polarization at $45^{\circ}$ to the $\mathrm{Cu}-\mathrm{O}$ bond direction for $x=0,0.05,0.10$, and 0.12 . Measurements were performed at $\mathrm{T}=200 \mathrm{~K}$ for $x=0$ and $\mathrm{T}=15 \mathrm{~K}$ for $x>0$. Raw data are shown as black solid lines and red line is the fit to a Gaussian. (e) Doping dependence of the $\mathrm{O} 2 p_{\pi}$ FWHM, with all samples grown using the high pressure synthesis shown as open circles, and undoped samples grown using the conventional ambient pressure technique shown as a solid square.

correlation effects. On the other hand, there is no appreciable spectral weight at energies above the top of the $\mathrm{O} 2 p_{\pi}$ band at $(\pi, \pi)$, even in the doped compounds. Therefore, the spectra from the $\mathrm{O} 2 p_{\pi}$ band can readily be compared between samples with different hole compositions.

In Figure 6.9, we show EDCs from $(\pi, \pi)$ from the $x=0,0.05,0.10$, and 0.12 samples, together with Gaussian fits to the data. To within experimental error, no sizable deviation from a purely Gaussian lineshape can be observed at all doping levels. This would seem to indicate that the relative contribution from electronelectron interactions to the photohole lifetime for the $\mathrm{O} 2 p_{\pi}$ state is reasonably small 
compared to electron-phonon interactions, even for the doped compositions. Note that the flat background at low binding energies $(\omega>-1.0 \mathrm{eV})$ persists even for finite $x$, allowing for a reliable fit and analysis of the raw data. A summary of the doping dependence of the $\mathrm{O} 2 p_{\pi}$ Gaussian FWHM is shown in Figure 6.9e. The most surprising finding from this analysis is that the FWHM of the $\mathrm{O} 2 p_{\pi}$ spectra from the undoped samples is narrower than those of the $x=0.05$ or 0.10 samples. If one considered the effects of electron-phonon interactions alone, one might expect a smooth decrease in the FWHM as the electron-phonon coupling strength is reduced monotonically with hole doping. However, this is not the case. The FWHM rises in going from $x=0$ to 0.05 , then appears to decrease monotonically from $x=0.05$ to 0.12. Moreover, the FWHM for the $x=0$ samples were obtained at $\mathrm{T}=200-300$ $\mathrm{K}$, while the FWHM for the $x>0$ samples were obtained at $\mathrm{T}=15 \mathrm{~K}$. Since the temperature dependence of the $\mathrm{O} 2 p_{\pi}$ FWHM is only weakly temperature dependent, we could assume that the $\mathrm{O} 2 p_{\pi}$ width should have essentially saturated near $\mathrm{T}=200$ $\mathrm{K}$, so that $\mathrm{FWHM}(10 \mathrm{~K}) \sim \operatorname{FWHM}(200 \mathrm{~K})$, as discussed in Chapter 5.

Here, we propose possible reasons for the increase in FWHM in going from $x=0$ to 0.05. Changes in the crystal chemistry should be taken into account when comparing the doped and undoped samples. An increase in the scattering rate from singleparticle scattering considerations (electrons scattering from microscopic Na dopants) should be on the $\sim 1-10 \mathrm{meV}$ scale, especially since the lineshape of the near- $\mathrm{E}_{\mathrm{F}}$ excitations becomes sharper with increasing $x$, and thus should be essentially irrelevant. Another consideration associated with crystal chemistry is that the doped compounds were synthesized using a high-pressure flux growth technique, while different batches of the undoped samples were grown using either the high-pressure technique or an ambient pressure approach. A small difference in the FWHMs were observed between the undoped samples grown under ambient or high-pressure, although this difference is well within the statistical error bars. This difference might be due to the fact that the larger crystals grown under ambient pressure tended to have flatter surfaces (sharper laser reflections) than the high-pressure crystals. Therefore, the more curved or warped surfaces from the high-pressure crystals might account for some of the additional broadening. However, even by assuming a fairly large angular broadening 
$\left( \pm 1^{\circ}\right)$ to the undoped samples, one cannot reasonably account for much additional energy broadening. Another potential concern in the doped samples is the effects of macroscopically inhomogeneous doping - because $\mu$ shifts rapidly with hole doping, variations in $x$ across a given sample could also result in a spread in $\mu$. For instance, $d \mu / d x$ was determined to be $-1.8 \mathrm{eV} \pm 0.5 \mathrm{eV} /$ hole. Allowing for a \pm 0.005 spread in $x$ across a given sample, this would correspond to an additional broadening of $\sim$ $18 \mathrm{meV}$. However, these additional corrections to the intrinsic width would not add directly, but roughly in quadrature. Therefore, if we assume an intrinsic width of 400 $\mathrm{meV}$, the combined effects of $\pm 1^{\circ}$ angular broadening and macroscopic chemical inhomogeneity listed above would only increase this to $\sim 405 \mathrm{meV}$. The sample-to-sample scatter in the undoped samples for $\Gamma_{\pi}$ is much larger than $\pm 5 \mathrm{meV}$, and $\Gamma_{\pi}$ of the $x=0.05$ sample is $490 \pm 55 \mathrm{meV}$. Either there exist additional extrinisc effects beyond our simple framework for accounting for macroscopic chemical or morphological broadening, or there else are other microscopic and intrinsic broadening mechanisms that we should take into account.

Here, we propose some potential microscopic explanations for this additional broadening in the doped samples. First, the above discussion neglected lifetime effects arising from electron-electron interactions. If the electron-electron scattering rate first increased very rapidly with doping from $x=0$ to 0.05 , and then saturated at higher doping levels, this might help to explain the sharp increase between the FWHM of the $x=0$ and 0.05 samples. If we convolute a Gaussian (FCB) with a $\mathrm{FWHM}=400 \mathrm{meV}$ with a Lorentzian (electron-electron) lineshape with a FWHM $=150 \mathrm{meV}$, the total resulting linewidth of the convolved function is $500 \mathrm{meV}$, but the lineshape remains rather close to a Gaussian (in this case, the Voigt parameter, $y=\Gamma_{\text {lor }} / \Gamma_{\text {gauss }} \sqrt{\ln 2} \sim 0.31$ ). Because of the asymmetric lineshape of the $\mathrm{O} 2 p_{\pi}$ peak, which limits us to only fitting the low-binding energy half of the peak, it is possible that an additional Lorentzian contribution could have been unaccounted for. Therefore, an additional contribution from increased electron-electron scattering in the doped samples is highly plausible, at least given the experimentally measured spectra. However, increasing the Voigt parameter beyond $y \sim 0.4-0.5$ begins to introduce significant deviations from a well-defined Gaussian lineshape. Another 
possibility is that of intrinsic microscopic carrier inhomogeneity. Recent scanning tunnelling spectroscopy (STS) measurements have shown apparent nanoscale variations in the local hole density (so-called "p-maps") [192]. The magnitude of these variations is significantly larger than what one would expect from variations in the chemical composition, on the order of $x$ of $\pm 0.01-0.02$. Taking this nanoscale variation into account in a worst case scenario, a variation of \pm 0.02 in the hole density might account for up to $\sim 72 \mathrm{meV}$ in broadening. However, added in quadrature to an intrinsic width of $400 \mathrm{meV}$, this would still only increase the total width to $\sim 410$ $\mathrm{meV}$. Therefore, more work (and a much greater number of doped samples) would be required to completely account for the sharp increase in the FWHM in going from $x=0$ to 0.05 . Neglecting the $x=0$ sample for the meantime, a clear decrease in the FWHM as a function of $x$ in the doped samples is evident in Figure 6.9. From a linear fit to the $x=0.05,0.10$, and 0.12 samples, we would estimate that $d \Gamma / d x$ to be $1.1 \pm 1.3 \mathrm{eV} /$ hole. This decrease in $\Gamma_{\pi}$ would be consistent with a weakening electron-phonon interaction for the $\mathrm{O} 2 p_{\pi}$ band.

We can attempt to estimate the doping dependent change in the electron-phonon coupling strength by relating $\Gamma_{\pi}(x) \rightarrow \Delta P(x)$, where $\Delta P(x)=g(x) \omega_{0}$ is the 0-0 to peak separation. We can use the $\mathrm{T}=0$ results of the independent boson model from Chapter 4 , since the measurements of the $x>0$ samples were performed at $15 \mathrm{~K}$ (whereas the boson energy $\omega_{0}>400 \mathrm{~K}$ ). From the analysis of the independent boson model, we can relate $\Gamma$ (the FWHM) to the separation of the 0-0 transition from the peak maximum. In the independent boson model, the separation is given by $g \omega_{0}$, while the FWHM, $\Gamma$, should be given by $\sqrt{8 \ln 2 g \omega_{0}^{2}}$. Therefore, the QP-to-centroid separation, $\Delta P$ should be given as $\Delta P=\Gamma^{2} /\left(8 \ln 2 \omega_{0}\right)$. In Figure 6.10 a, we show $\Delta P(x)$ using the experimental values of $\Gamma_{\pi}$ from Figure 6.9 and $\omega_{0}=40 \mathrm{meV}$. In fact, we can see that $\Delta P(x)$ changes faster than even $\mu(x)$ shown in Figure 6.2, with a value of approximately of $d \Delta P / d x \sim-4.5 \pm 5.7 \mathrm{eV} /$ hole. However, this quantitative value of $\Delta P(x)$ depends sensitively on the absolute value of $\Gamma$, as well as the choice of $\omega_{0}$. Moreover, the error bars for our determination of $\Gamma(x)$ are rather large, and more comprehensive experiments are necessary before one can make strong claims as to the doping dependence of $\Gamma$ and $\Delta P$, as well as an accurate value for the absolute 

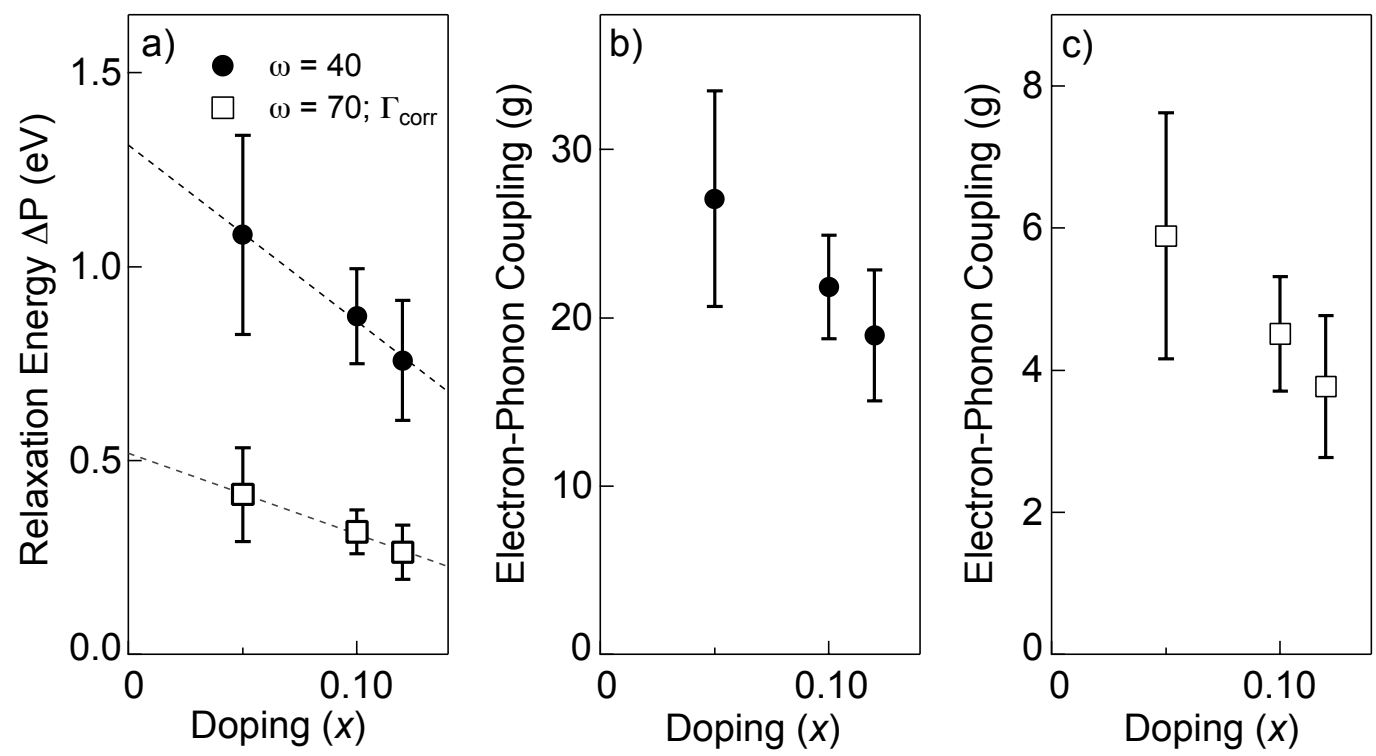

Figure 6.10: (a) Estimation of the polaron binding energy of the $\mathrm{O} 2 p_{\pi}$ state extracted from the FWHM and independent boson model, using phonon frequencies of $\omega_{0}=40$ and $70 \mathrm{meV}$, respectively. Electron-phonon coupling constant, $g(x)$ (representative of the average number of phonons dressing a photohole, $\langle n\rangle)$, as a function of doping using $\omega_{0}=40 \mathrm{meV}$ (b) and $70 \mathrm{meV}$ (c), respectively.

width of $\Gamma$ in the doped samples. In Figure 6.10a, we also plot $d \Delta P / d x$ using $\omega_{0}=70$ meV (taking, for instance, the oxygen half-breathing mode) and subtract a nominal "extrinsic broadening contribution" to the linewidth, such that $\Gamma_{\pi}(0.05)=400 \mathrm{meV}$. Using these new values, we find that $d \Delta P / d x \sim-2.1 \pm 2.6 \mathrm{eV} /$ hole.

This doping dependent change in the $\mathrm{O} 2 p_{\pi}$ state raises an important question with our earlier analysis of the doping dependence of $\mu$. The peak position of the $\mathrm{O} 2 p_{\pi}$ band was used as a reference marker for measuring $d \mu / d x$. However, later analysis showed that even the $\mathrm{O} 2 p_{\pi}$ state was not just a simple band-like state, but also exhibited $\mathrm{FCB}$, and therefore the $\mathrm{O} 2 p_{\pi}$ peak maximum did not represent the QP pole. Moreover, the fact that $\Gamma_{\pi}$ shows significant doping dependence might imply that we should also account for this in our determination of $d \mu / d x$, since the peak maximum of the $\mathrm{O} 2 p_{\pi}$ should not be the position of the QP pole. However, the analysis of the independent boson model in Chapter 4 suggests that even in the presence of strong electron-phonon coupling, the centroid of incoherent spectral weight 
coincides exactly with the energy position of the bare, frozen electronic band. This is also confirmed by the theoretical studies discussed in Chapter $5[141,170]$. Unless the 0-0 transition at the top of the $\mathrm{O} 2 p_{\pi}$ band extends all the way to $\mu$ and controls the chemical potential shift (and not the LHB), we should still regard the O2p $p_{\pi}$ peak maximum as a good reference for the shift of $\mu$. As we have shown in Figure 6.10, the quantitative value of $\Delta P$ depends sensitively on the absolute value of $\Gamma$, as well as the choice of $\omega_{0}$, neither of which has been firmly established. Nevertheless Figure 6.10 , this still clearly establishes that the $\mathrm{O} 2 p_{\pi} 0-0$ transition is well separated from $\mathrm{E}_{\mathrm{F}}$ by at least $1 \mathrm{eV}$, and therefore should not affect $d \mu / d x$, even in the presence of polaron formation.

We note that it is unclear whether we can generalize about the particular strength of electron-phonon interactions in the LHB from our studies of the $\mathrm{O} 2 p_{\pi}$ state. Obviously, the electron-phonon interactions for the $\mathrm{O} 2 p_{\pi}$ should be rather different than the LHB. Since the two electronic states are fundamentally different, the $\mathrm{O} 2 p_{\pi}$ may be coupled strongly to different particular phonon modes, and the matrix element for electron-phonon coupling for those particular states may well be rather different from the LHB and its phonon modes. This difference could potentially be addressed by studying ab initio calculations which use electronic structure calculations in conjunction with a detailed shell model obtained from a refinement of the neutron scattering data, as has already been attempted by Rösch and Gunnarsson for $\mathrm{La}_{2} \mathrm{CuO}_{4}$ [193]. Unfortunately, such detailed neutron scattering studies of the phonon modes in $\mathrm{Sr}_{2} \mathrm{CuO}_{2} \mathrm{Cl}_{2}$ or $\mathrm{Ca}_{2} \mathrm{CuO}_{2} \mathrm{Cl}_{2}$ have not yet been attempted, making such a direct comparison currently impossible. For instance, it would be interesting to see if the much larger width of the $\mathrm{O} 2 p_{\pi}$ peak could be explained from larger intrinsic coupling of the $\mathrm{O} 2 p_{\pi}$ band to particular phonon modes. 


\section{Chapter 7}

\section{Evolution of the Fermi Surface in $\mathrm{Ca}_{2-x} \mathrm{Na}_{x} \mathrm{CuO}_{2} \mathrm{Cl}_{2}$}

One of the crucial elements in the physics of the doped Mott insulator is understanding how the low-lying excitations emerge throughout k-space, a task uniquely suited to ARPES ${ }^{1}$. Precisely at half-filling, there is no FS to speak of, because the LHB is completely filled and $\mu$ sits somewhere within the gap. There has been previous work on the observation of a so-called "remnant Fermi surface" in undoped $\mathrm{Ca}_{2} \mathrm{CuO}_{2} \mathrm{Cl}_{2}$ [88], although this feature is not a real FS, but associated with the distribution of spectral weight in $\mathbf{k}$-space with respect to the first and second AF Brillouin zones. One can imagine two different simplified scenarios for the evolution of the FS as one dopes the Mott insulator. The first is to treat hole doping of the LHB similar to doping a band insulator. In this picture, $\mu$ shifts into the lower Hubbard band defined by the $t-J$ model in Figure 4.6, and the upper Hubbard band remains completely unoccupied and the Mott gap remains intact. In this case, one might expect to see a small hole pocket emerge centered at $(\pi / 2, \pi / 2)$ with a volume proportional to $x$. The second scenario is that one can imagine that upon doping, the upper and lower Hubbard bands merge as the Mott gap collapses, and one is left with a case similar to the noninteracting electron picture where electron correlations are turned off. In

\footnotetext{
${ }^{1} \mathrm{~A}$ large portion of the work discussed in this chapter has been published in K.M. Shen et al., Science 307, 901 (2005).
} 
a)

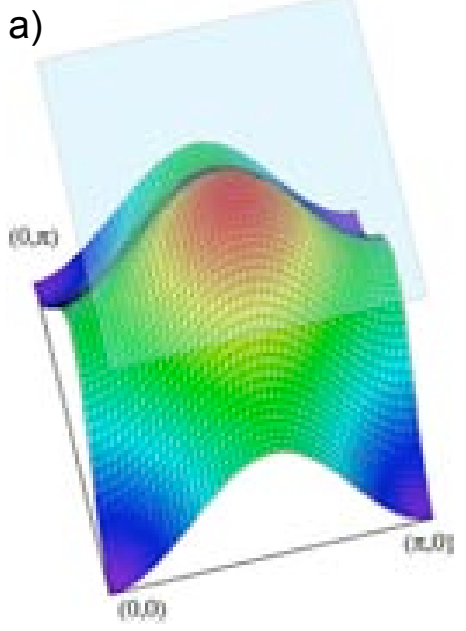

b)

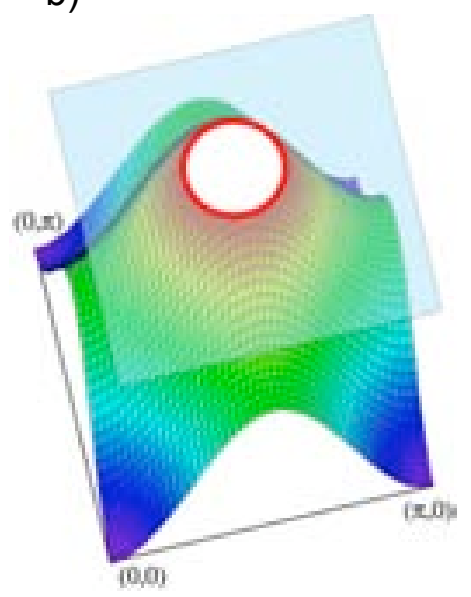

c)

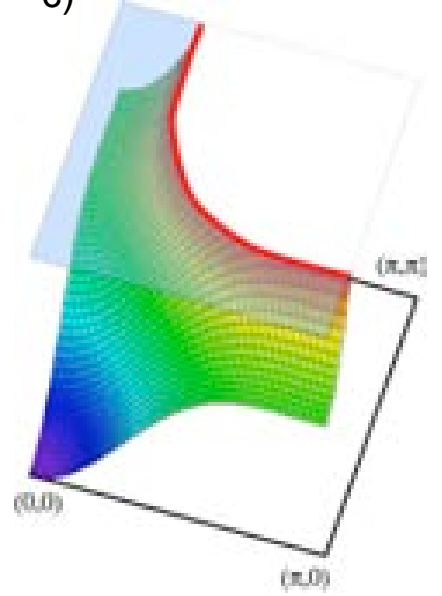

Figure 7.1: (a) 3D plot of theoretical dispersion of the lower Hubbard band in undoped Mott insulator. (b) Rigid doping into the lower Hubbard band, resulting in a small hole pocket centered at $(\pi / 2, \pi / 2)$. (c) Development of a non-interacting band-like FS with a volume of $1-x . \mathrm{E}_{\mathrm{F}}$ is denoted by the transparent window-like surface, and the FS is shown as a thick red line.

this case, one would expect to recover a large, hole-like $1-x$ FS centered at $(\pi, \pi)$.

These two cases are illustrated in Figure 7.1, where we start from the case of the undoped Mott insulator (where the 2D dispersion is shown as a three-dimensional surface where $E$ is the vertical axis) in Figure 7.1a. Rigidly dropping $\mu$ into this lower Hubbard band would give us the small hole pocket shown in Figure 7.1b, while the non-interacting band picture would give us a contour reminiscent of 7.1c. In a picture where the physics can be described purely as a doped Mott insulator, one might expect the formation of a small hole pocket as in Figure 7.1b at very low hole concentrations. This possibility has been explored theoretically using RVB or gauge theories which attempt to account for the Hubbard $U$ in some fashion $[194,195,196]$. In addition to the large $1-x$ FS and the small $x$ hole pocket, the possibility of Fermi surface truncation (so-called "Fermi arcs") due to highly k-anisotropic scattering rates into "hot" and "cold" spots has been discussed, primarily by N. Furukawa, T.M. Rice, and coworkers $[197,198,199]$. Finally, work by S.A. Kivelson and coworkers, and R.S. Markiewicz has discussed the possible FS which arises from these spatially 

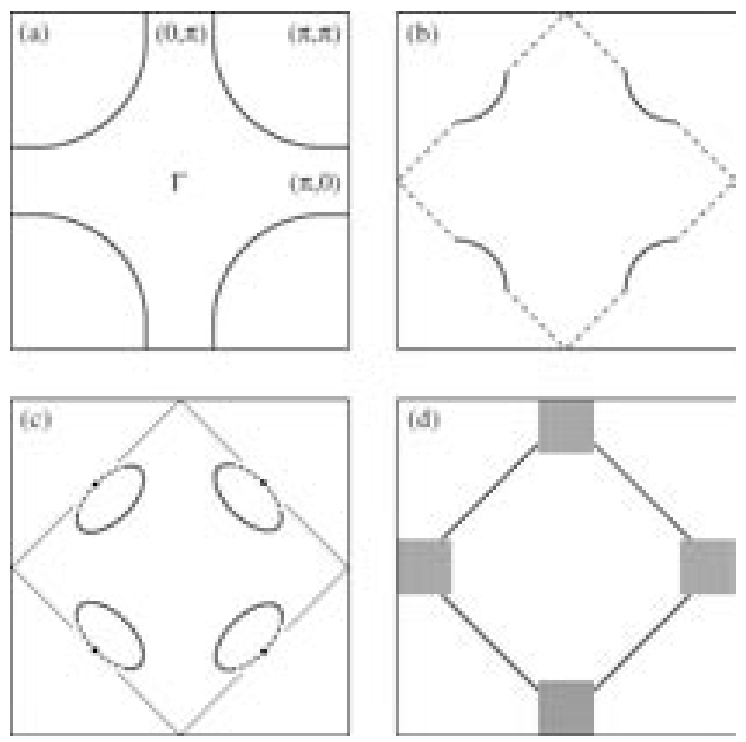

Figure 7.2: (a) Large $1-x$ band-like FS. (b) Fermi arcs, truncated at the antiferromagnetic zone boundary by umklapp scattering, resulting in hot and cold spots. (c) Small, $x$ hole pockets. (d) Possible E $E_{F}$ spectral weight arising from disordered stripes. From [40].

inhomogeneous charge distributions or "stripes" (which sometimes appear to conspire to give the appearance of the FS similar to that expected from band theory) [200, 201]. These pictures are shown in Figure 7.2.

Our work in Chapters 4, 5, and 6 makes it evident that because of polaronic effects, the QP states are far separated in energy from the original "frozen lattice" LHB states. Therefore, the well-separated QP states are not fixed to the original frozen lattice dispersion of the LHB, and can potentially form all the different forms of the FS shown in Figure 7.2. Even without the added complication of small polaron formation, there was little theoretical consensus on the evolution of the Fermi surface when taking into account solely electronic considerations. However, it may be possible that while lattice effects strongly affect the distribution of spectral weight in $\mathcal{A}(\mathbf{k}, \omega)$ as a function of $\omega$, polaronic effects may not strongly alter the $\mathbf{k}$-distribution of spectral weight, and may not shift the underlying FS, although this is still not a well understood problem. 


\subsection{Low-Lying Spectral Weight in $\mathrm{Ca}_{2-x} \mathrm{Na}_{x} \mathrm{CuO}_{2} \mathrm{Cl}_{2}$}

To determine the FS of $\mathrm{Ca}_{2-x} \mathrm{Na}_{x} \mathrm{CuO}_{2} \mathrm{Cl}_{2}$, we plot the low-lying spectral weight within a narrow window near- $\mathrm{E}_{\mathrm{F}}( \pm 10 \mathrm{meV})$ for all three different doping levels. We should note that in Fermi liquid systems, the Fermi surface has a precise and rigorous meaning. In systems such as the lightly doped cuprates, which are potentially non-Fermi liquid-like and may not possess truly well-defined Landau quasiparticle excitations, it is not correct to call the contour of spectral weight at $\mathrm{E}_{\mathrm{F}}$ a real "Fermi surface". However, solely for convenience, we will continue to call the contour of near$E_{F}$ spectral weight the "Fermi surface", keeping in mind that this may not rigorously correct. Data for $\mathrm{Ca}_{1.9} \mathrm{Na}_{0.10} \mathrm{CuO}_{2} \mathrm{Cl}_{2}$ was previously published in $[127,202]$. The intensity plots in Figure 7.3 were taken at $15 \mathrm{~K}$ with $h \nu=25.5 \mathrm{eV}$ and polarization at $45^{\circ}$ to the $\mathrm{Cu}-\mathrm{O}$ bond direction. To normalize the data, we utilized the relatively flat high-energy background at $\sim-1 \mathrm{eV}$. To compare the low-energy intensity between samples, we not only checked against this background at $-1 \mathrm{eV}$, but we also used the valence band features as a point of comparison, since our earlier work demonstrated that the intensity and shape of the gross valence band features are relatively insensitive to carrier doping. Both approaches give effectively the same result, which can be seen in Figure 7.3, where the overall intensity grows as a function of $x$. As shown in Figure 7.3, the distribution of spectral weight is also highly anisotropic, and is maximum along the $(0,0)-(\pi, \pi)$ nodal line and drops off precipitously towards the antinodes. Although photoelectron matrix element effects can greatly affect the

k-distribution of spectral weight, we found that this strong anisotropy did not vary significantly over multiple Brillouin zones, or with polarizations at $0^{\circ}$ and $45^{\circ}$ to the $\mathrm{Cu}-\mathrm{O}$ bond, or at different photon energies between 16.5-32 eV, strongly suggesting that this is an intrinsic effect.

From the data in this figure, it appears that the contour of low-lying spectral weight stretches from the nodal direction towards the antinodes as $x$ is increased. However, this is somewhat misleading due to the cutoff of the 2D false color intensity plots. A more informative way to view the low-energy spectral intensity is with a 

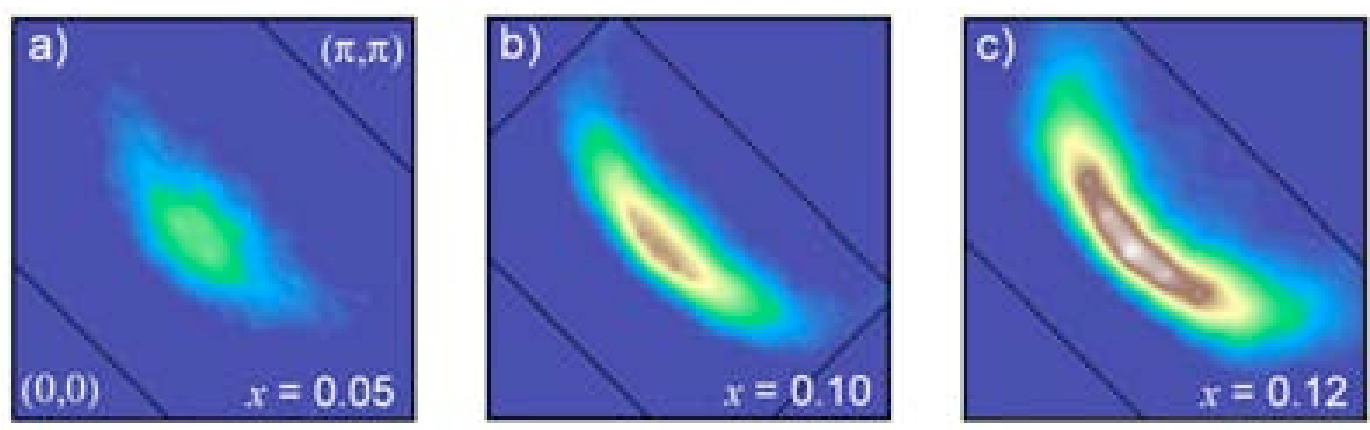

Figure 7.3: False color intensity scale plots over the Brillouin zone quadrant of $\mathrm{Ca}_{2-x} \mathrm{Na}_{x} \mathrm{CuO}_{2} \mathrm{Cl}_{2}$ of the integrated spectral weight within a $\pm 10 \mathrm{meV}$ window around $\mathrm{E}_{\mathrm{F}}$ for $x=0.05,0.10$, and 0.12 compositions (a-c). Data acquisition range is shown within the black lines. Data were taken at $15 \mathrm{~K}$ with $h \nu=25.5 \mathrm{eV}$ photons and a polarization at $45^{\circ}$ to the $\mathrm{Cu}-\mathrm{O}$ bond direction. Data were symmetrized about the $(0,0)-(\pi, \pi)$ line, and normalized to the featureless background at high binding energies $(\sim-1 \mathrm{eV})$.

"bird's eye view" plot of the spectral weight, where the near- $E_{\mathrm{F}}$ intensity is plotted as height along the $z$-axis, in Figure 7.4. In these plots, the raw experimental data are not shown, but rather curves generated from parameterized fits of $\mathbf{k}_{\mathrm{F}}$, the angular intensity, and the angular width. Nevertheless, this should be a very accurate representation of the raw spectral intensity in Figure 7.3. From these plots, one can see that the angular distribution of spectral weight does not change appreciably, but it is primarily the overall intensity that scales with $x$. One can see in going from $x$ $=0.05$ to 0.12 , the overall intensity increases, but the contour of intensity remains roughly constant.

This strong anisotropy in the spectral weight distribution of $\mathrm{Ca}_{2-x} \mathrm{Na}_{x} \mathrm{CuO}_{2} \mathrm{Cl}_{2}$ is reminiscent of lightly doped $\mathrm{La}_{2-x} \mathrm{Sr}_{x} \mathrm{CuO}_{4}$ [171] and even heavily underdoped $\mathrm{Bi}_{2} \mathrm{Sr}_{2} \mathrm{CaCu}_{2} \mathrm{O}_{8+\delta}$ [106]. All lightly hole doped cuprates to date have shown that well-defined QP-like excitations first emerge near $(\pi / 2, \pi / 2)$, while the antinodal excitations appear broad and indistinct, until about optimal doping. This generic behavior across materials would also argue against a trivial matrix element effect causing this anisotropy in spectral weight, since it is known that different materials 

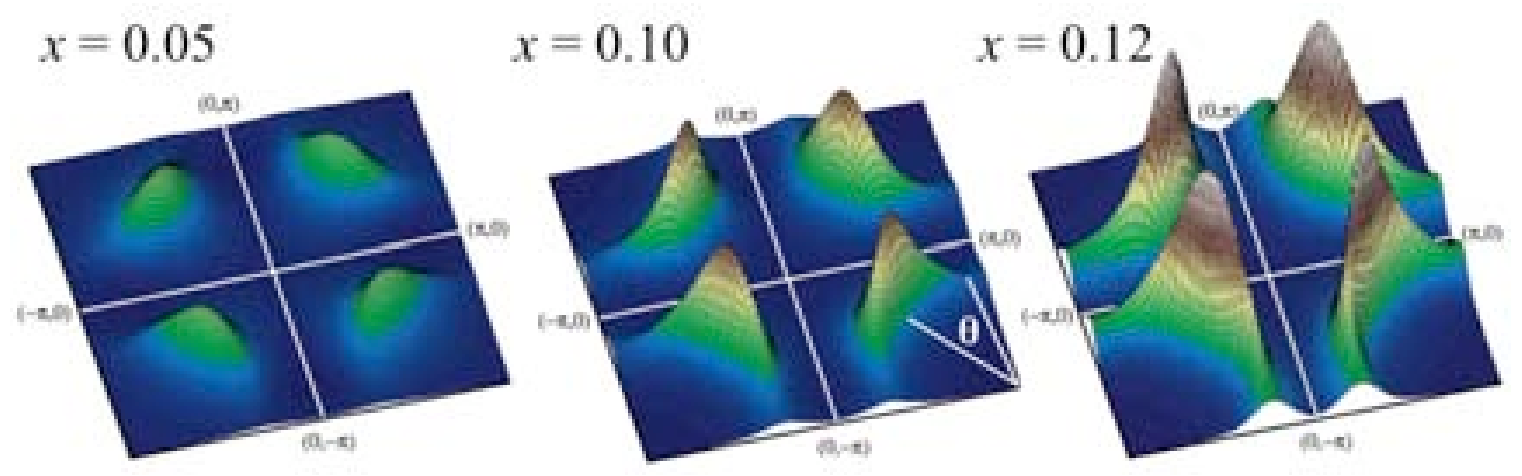

Figure 7.4: 3D bird's eye view plots of the spectral intensity within a $\pm 10 \mathrm{meV}$ window, as shown in Figure 7.3. These plots were generated by parameterizing the angular distribution of spectral weight, the width of the EDC plots, and the underlying FS. These plots demonstrate that the apparent Fermi arcs do not rigidly extend with doping, but it is more representative of an overall growth in spectral weight.

typically have very different photoelectron matrix elements which depend very sensitively on the details of the crystal structure. To better quantify this, we show the angular distribution of spectral weight along the ostensible FS, where $\theta$ is defined in Figure 7.4b. Essentially, this is the $z$-position (height) along the top of the "ridge" in Figure 7.4, as a function of $\theta$. This is shown in Figure 7.5 where the near- $\mathrm{E}_{\mathrm{F}}$ angular intensity is plotted as a function of $\theta$ for the three doping compositions. In addition, we plot similar data from $\mathrm{La}_{2-x} \mathrm{Sr}_{x} \mathrm{CuO}_{4}$ taken from [171] for the purpose of comparison. The relative intensities for the different $\mathrm{La}_{2-x} \mathrm{Sr}_{x} \mathrm{CuO}_{4}$ compositions were matched to the comparable doping levels for $\mathrm{Ca}_{2-x} \mathrm{Na}_{x} \mathrm{CuO}_{2} \mathrm{Cl}_{2}$. In Figure 7.5b, we show the intensity of the three doping levels scaled to the nodal value (normalized to one) which show the quantitatively similar angular profiles of spectral weight. In Figure 7.5c, we show the doping dependence of the total low-lying FS intensity $\left( \pm 10 \mathrm{meV}\right.$ ) for $\mathrm{Ca}_{2-x} \mathrm{Na}_{x} \mathrm{CuO}_{2} \mathrm{Cl}_{2}$ and $\mathrm{La}_{2-x} \mathrm{Sr}_{x} \mathrm{CuO}_{4}$. For $\mathrm{Ca}_{2-x} \mathrm{Na}_{x} \mathrm{CuO}_{2} \mathrm{Cl}_{2}$, we calibrated the near- $\mathrm{E}_{\mathrm{F}}$ weight by normalizing against the valence band, or the flat background at $\sim-1 \mathrm{eV}$, as described earlier. At low dopings, the FS intensity appears to be $I \propto x$, but appears to increase superlinearly with $x$ at higher dopings. Recent work on $\mathrm{Bi}_{2} \mathrm{Sr}_{2} \mathrm{CaCu}_{2} \mathrm{O}_{8+\delta}$ [106] also appears to be roughly consistent with $\mathrm{La}_{2-x} \mathrm{Sr}_{x} \mathrm{CuO}_{4}$ and $\mathrm{Ca}_{2-x} \mathrm{Na}_{x} \mathrm{CuO}_{2} \mathrm{Cl}_{2}$. These similarities would appear to suggest an 

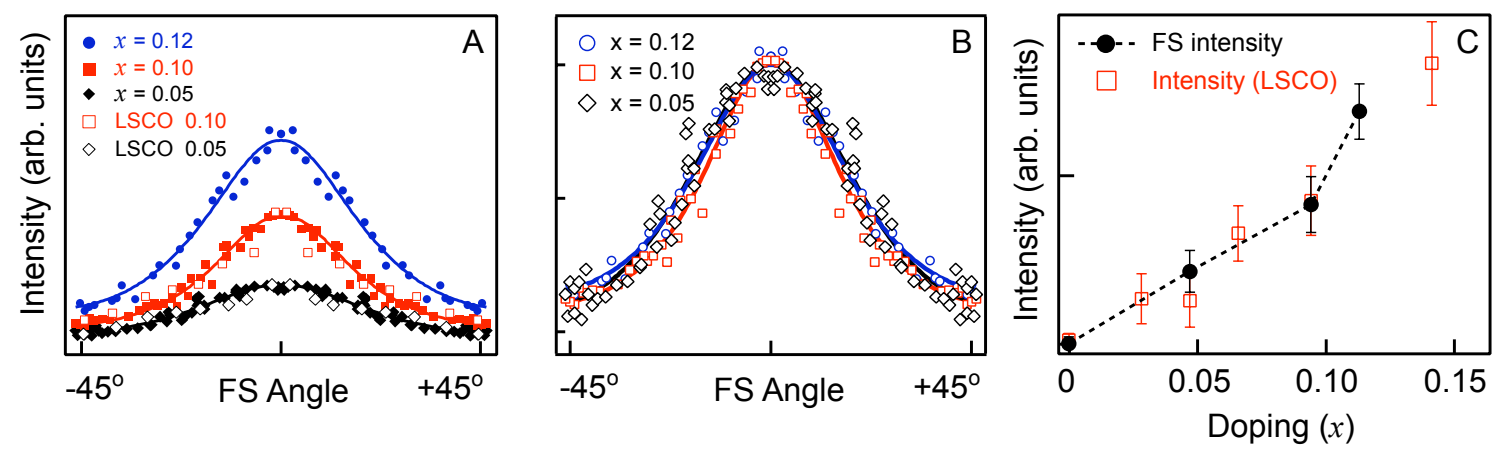

Figure 7.5: (a) Angular distribution of spectral weight as a function of angle along the FS. Data from $\mathrm{Ca}_{2-x} \mathrm{Na}_{x} \mathrm{CuO}_{2} \mathrm{Cl}_{2}$ are plotted as solid symbols, while data from $\mathrm{La}_{2-x} \mathrm{Sr}_{x} \mathrm{CuO}_{4}$, from [171], are plotted as open symbols. (b) Same angular distributions from $\mathrm{Ca}_{2-x} \mathrm{Na}_{x} \mathrm{CuO}_{2} \mathrm{Cl}_{2}$ in (a), but normalized to the same intensity, showing the quantitatively similar angular distributions. (c) Doping dependence of FS intensity which as a function of $x$ for $\mathrm{Ca}_{2-x} \mathrm{Na}_{x} \mathrm{CuO}_{2} \mathrm{Cl}_{2}$ (black circles) and $\mathrm{La}_{2-x} \mathrm{Sr}_{x} \mathrm{CuO}_{4}$ (red squares).

intrinsic commonality in the low-lying excitations between different cuprate families. This might also imply a generic microscopic origin for the low-lying nodal states irrespective of material-specific parameters and ordering tendencies (such as the tendency to form one-dimensional "static stripes" [29]).

As discussed earlier, this generic suppression of antinodal weight would again suggest that this is an intrinsic property of the single-electron spectral function of the lightly doped cuprates and not a trivial matrix element effect or a material-specific feature. We can also demonstrate this by showing EDCs around the FS, as shown in Figure 7.6 for all three doping levels. From the EDCs, it becomes apparent that the anisotropy observed in Figure 7.3 arises not from overall matrix element suppression or a pure $d$-wave gap, but the lack of coherent QP weight at the antinodes, and we will discuss this nodal/antinodal dichotomy in further detail. 


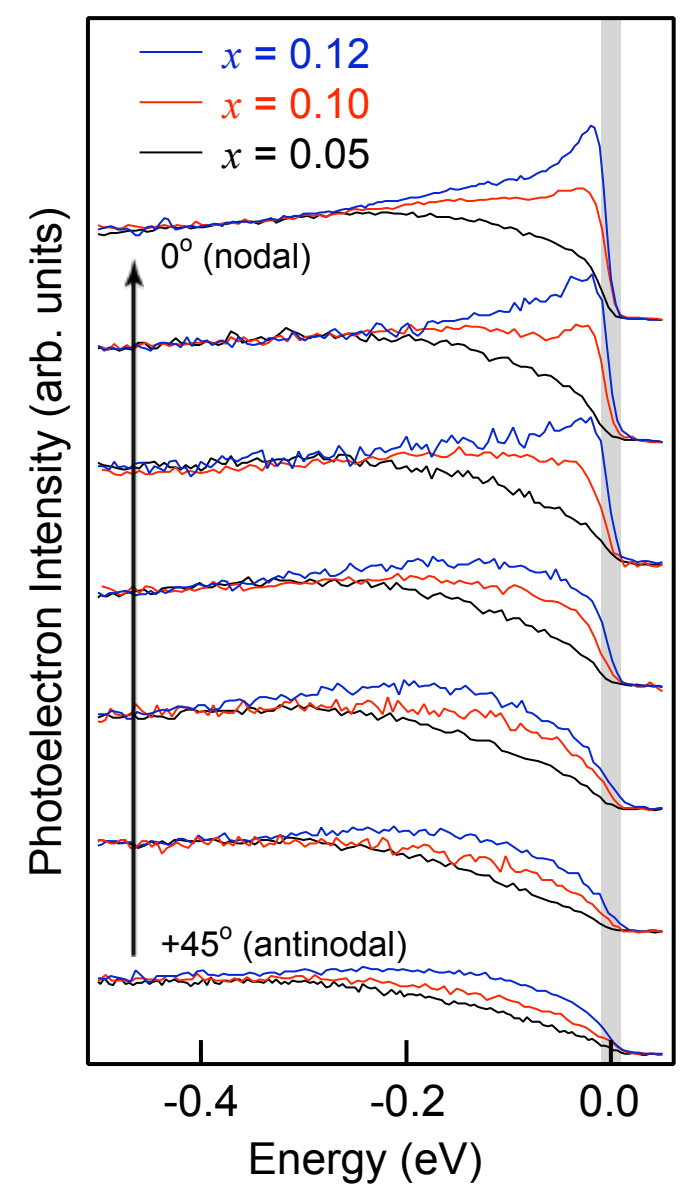

Figure 7.6: EDCs as a function of angle (as defined in Figure 7.4) around the Fermi surface of $\mathrm{Ca}_{2-x} \mathrm{Na}_{x} \mathrm{CuO}_{2} \mathrm{Cl}_{2}$ at $x=0.05,0.10$, and 0.12 .

\subsection{Determining the "Fermi Surface" in $\mathrm{Ca}_{2-x} \mathrm{Na}_{x} \mathrm{CuO}_{2} \mathrm{Cl}_{2}$}

\subsubsection{Methodology for Quantifying the Fermi Surface}

In a Fermi liquid, the FS can be defined as where the momentum distribution $n(\mathrm{k})$ has a discontinutity at $\mathrm{T}=0$; the magnitude of this discontinuity corresponds to the QP residue. However, even in a Luttinger liquid (LL) where FL quasiparticles do not exist due to fractionalization of the single electron excitations into separate 

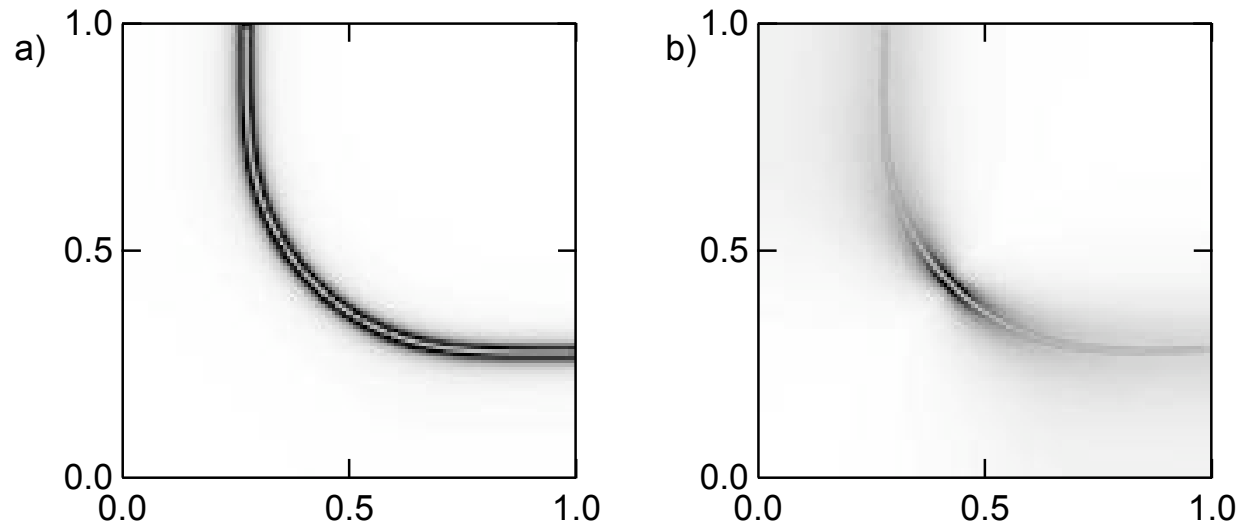

Figure 7.7: (a) Simulation of Fermi-liquid-like $\mathcal{A}(\mathbf{k}, \omega)$ (intensity plot) and MDCdetermined FS (circles), showing excellent agreement between actual FS and MDCderived $\mathbf{k}_{\mathrm{F}}$ s. (b) Simulation of same underlying FS, but with a $d$-wave BCS gap and anisotropic self-energy. MDC-determined $\mathbf{k}_{\mathrm{F}} \mathrm{S}$ still demonstrate excellent agreement with (a) and the underlying FS

topological defects, one can still define a sort of underlying FS. For the LL, one can use a criterion of $\mathbf{k}_{\mathrm{F}}$ as being where $|\nabla n(\mathrm{k})|$ diverges at $\mathrm{T}=0$. From an experimental standpoint, using $n(\mathrm{k})$ is very difficult to implement accurately from a practical point of view, although it has been employed previously [88]. There are a number of issues with using $n(\mathrm{k})$, including possible strong $\mathbf{k}$-dependence of matrix elements, the need to integrate over very wide energy ranges particularly for strongly correlated systems (impractical, because of the presence of other unrelated bands), and the influence of the background intensity. Another method to determine the position of a particular $\mathbf{k}_{\mathrm{F}}$ is to examine each EDC and estimate whether the QP pole is above or below $\mathrm{E}_{\mathrm{F}}$. This particular approach was utilized primarily in the earlier days of ARPES (before 2000) before the advent of the 2D multiplexing detectors, when EDCs were taken one at a time. The difficulty with this approach is that it either uses a somewhat arbitrary metric (such as the leading edge midpoint) or requires extensive modeling of the spectral function. As a result, the EDC method is rather subjective, although it can yield very accurate results if implemented correctly. Another practical consideration is that with modern electron analyzers producing $\sim 10^{4}$ spectra, the EDC method becomes extemely slow and time-consuming. 
Another method which is much more practical for analyzing a large array of spectra is the MDC method. The principle behind the MDC method is to look for maxima in an MDC at $\mathrm{E}_{\mathrm{F}}$ which passes through the FS, since the spectral weight at $\mathrm{E}_{\mathrm{F}}$ should be maximum when the $\mathrm{QP}$ pole sits at $\mathrm{E}_{\mathrm{F}}$. The $\mathbf{k}$ position of this maximum should then correspond to $\mathbf{k}_{\mathrm{F}}$. The benefit of this technique is that it provides a relatively unbiased and mechanical procedure for extracting the FS. In fact, in the absence of strong matrix element or other complicating effects, the MDC method can extract the underlying (normal-state) FS, even in gapped systems. In Figure 7.7, we show the spectral intensity at $\mathrm{E}_{\mathrm{F}}$ of a simulated $\mathcal{A}(\mathbf{k}, \omega)$. In Figure $7.7 \mathrm{a}$ we show the $\mathrm{E}_{\mathrm{F}}$ intensity of our simulation assuming a Fermi liquid-like self-energy and a finite impurity scattering term. The white circles are the values of $\mathbf{k}_{\mathrm{F}}$ determined from MDC extraction. The values of $\mathbf{k}_{\mathrm{F}}$ from the MDC extraction agree exactly with the actual FS, as can be seen directly. In Figure $7.7 \mathrm{~b}$, we show another simulated $\mathcal{A}(\mathbf{k}, \omega)$ where we use the same FS, but add a $d$-wave gap (of the BCS form) into $\mathcal{A}(\mathbf{k}, \omega)$, along with a strongly $\mathbf{k}$-anisotropic self-energy. This was intended to approximate the experimental situation as closely as possible. We then implemented the MDC $\mathbf{k}_{\mathrm{F}}$ extraction method on this simulated data and obtained essentially the same noninteracting FS shown in Figure 7.7a. However, for Figure 7.7b, this is not the "true" FS (for the $d$-wave gapped system, the FS is simply a nodal point), but rather the underlying normal-state FS. The reason for this is that the MDC maximum corresponds to the position where the gapped band approaches most closely to $\mathrm{E}_{\mathrm{F}}$, and for the BCS case, this position corresponds to $\mathbf{k}_{\mathrm{F}}$ for the normal-state FS. In addition to our simulations, the MDC determination method has also proven very successful for determining the FS in simpler systems, such as the idealized 2D Fermi liquid system (above $1.5 \mathrm{~K}$ ), $\mathrm{Sr}_{2} \mathrm{RuO}_{4}[121,122,203]$. $\mathrm{In}_{\mathrm{Sr}_{2}} \mathrm{RuO}_{4}$, there are 4 electrons in $3 \mathrm{Ru} 4 d$ $t_{2 g}$ bands. Calculating the Luttinger volume enclosed by the $\alpha, \beta$, and $\gamma$ FS sheets, we find excellent agreement of our MDC-derived volumes to within $2 \%$ of the known value of 4 electrons / unit cell. Nevertheless, the situations outlined above for Figure 7.7 and $\mathrm{Sr}_{2} \mathrm{RuO}_{4}$ are examples where $Z>0$, i.e. where the quasiparticle concept is still applicable. It remains to be seen how appropriate the MDC methodology is for the case of non-Fermi liquids or polaronic systems where $Z \ll 1$, such that the 


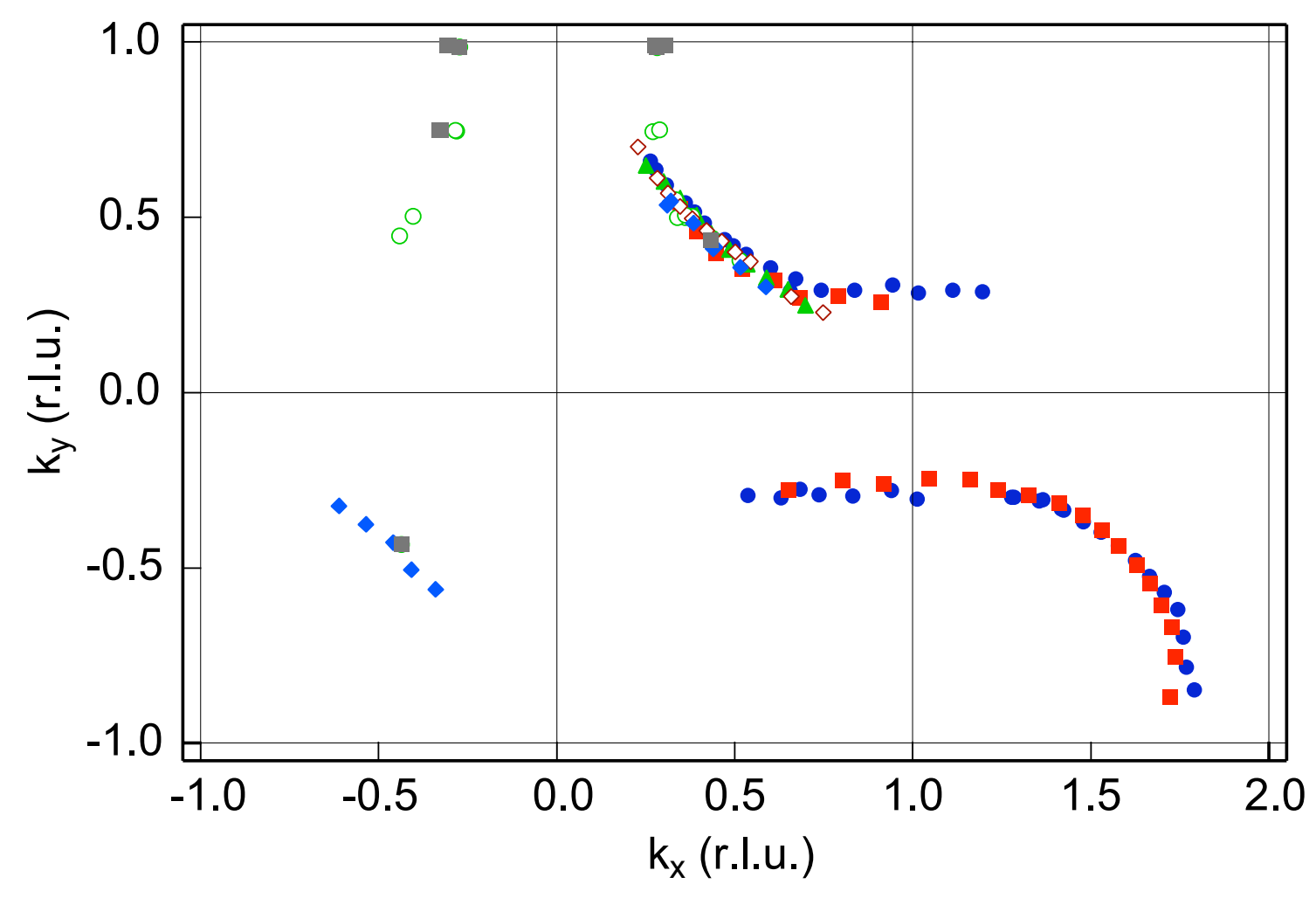

Figure 7.8: Extracted $\mathbf{k}_{\mathrm{F}} \mathrm{s}$ from $\mathrm{Ca}_{1.9} \mathrm{Na}_{0.10} \mathrm{CuO}_{2} \mathrm{Cl}_{2}$ using the MDC methodology. Data were collected on multiple samples (shown in different symbols) over multiple Brillouin zones.

incoherent spectral weight dominates, even near $\mathrm{E}_{\mathrm{F}}$.

\subsubsection{Application of MDC Methodology to $\mathrm{Ca}_{2-x} \mathrm{Na}_{x} \mathrm{CuO}_{2} \mathrm{Cl}_{2}$}

In Figure 7.8, we show in multiple Brillouin zones $\mathbf{k}_{\mathrm{F}} \mathrm{s}$ determined from the MDC methodology, taken from multiple samples of $\mathrm{Ca}_{1.9} \mathrm{Na}_{0.10} \mathrm{CuO}_{2} \mathrm{Cl}_{2}$. All data were acquired at $\mathrm{T}=15 \mathrm{~K}$, but under different experimental conditions. Spectra were taken with the polarization either parallel to or at $45^{\circ}$ to the $\mathrm{Cu}-\mathrm{O}$ bonds, and with the Scienta analyzer slits either along the $\mathrm{Cu}-\mathrm{O}$ bond direction or along the $\mathrm{Cu}-\mathrm{O}$ diagonal. Data were also acquired at different photon energies in the range of 16.5 - $28 \mathrm{eV}$, although most data were acquired using $h \nu=21.2$ or $25.5 \mathrm{eV}$, and include data both from a He I plasma discharge lamp and (primarily) synchrotron radiation. 
Because of errors in sample alignment and matrix element effects, we combined $\mathbf{k}_{\mathrm{F}} \mathrm{s}$ measured from multiple samples under different experimental conditions to attempt to minimize the aforementioned sources of error. We believe the effects due to $\mathbf{k}_{z}$ dispersion are minimal, since the resistivity anisotropy $\rho_{c} / \rho_{a b} \sim 10^{4}$. There has recently been work discussing the effects of $\mathbf{k}_{z}$ broadening in $\mathrm{La}_{2-x} \mathrm{Sr}_{x} \mathrm{CuO}_{4}$ and $\mathrm{Bi}_{2} \mathrm{Sr}_{2} \mathrm{CaCu}_{2} \mathrm{O}_{8+\delta}$ [204]. However, in $\mathrm{La}_{2-x} \mathrm{Sr}_{x} \mathrm{CuO}_{4}$ the anisotropy $\left(\sim 10^{-3}\right)$ is much less than in $\mathrm{Ca}_{2-x} \mathrm{Na}_{x} \mathrm{CuO}_{2} \mathrm{Cl}_{2}$, while in $\mathrm{Bi}_{2} \mathrm{Sr}_{2} \mathrm{CaCu}_{2} \mathrm{O}_{8+\delta}$, one must distinguish between intracell and intercell hopping. Nevertheless, any $\mathbf{k}_{z}$ broadening should drop to zero along the nodal direction, so it is primarily along the antinodal direction that one must be most concerned about possible $\mathbf{k}_{z}$ effects.

To quantify the ostensible "Fermi surfaces" of $\mathrm{Ca}_{2-x} \mathrm{Na}_{x} \mathrm{CuO}_{2} \mathrm{Cl}_{2}$, we took the $\mathbf{k}_{\mathrm{F}} \mathrm{S}$ from Figure 7.8 and translated them by $\mathbf{G}$-vectors into a reduced zone scheme. We then assumed tetragonal symmetry and fourfold symmetrized every data point into all four quadrants of the first Brillouin zone. Finally, we generated a mirror image by reflecting all points across the $(0,0)-(\pi, \pi)$ high symmetry line, thereby placing all $\mathbf{k}_{\mathrm{F}} \mathrm{S}$ within the irreducible wedge of the $2 \mathrm{D}$ tetragonal Brillouin zone. This procedure was repeated for $x=0.05$ and 0.12 compositions as well, yielding the FS maps shown in Figure 7.9. The data in shown in Figure 7.3 only represents data from one sample at each composition while the points in Figure 7.9 are typically representative of data from $\sim 5$ samples

From the data points in Figure 7.9, we can make a fit to the experimental $\mathbf{k}_{\mathrm{F}} \mathrm{s}$ by using a tight-binding fit to the FS. We use a functional form:

$E=-2 t\left(\cos \left(k_{x}\right)+\cos \left(k_{y}\right)\right)-4 t^{\prime}\left(\cos \left(k_{x}\right) \times \cos \left(k_{y}\right)\right)-2 t^{\prime \prime}\left(\cos \left(2 k_{x}\right)+\cos \left(2 k_{y}\right)\right)+\mu$

We give the following parameters in units of $t$, such that $t=1$. For $x=0.05, t^{\prime}$ $=-0.162, t^{\prime \prime}=0.151$, and $\mu=-0.162$. For $x=0.10, t^{\prime}=-0.276, t^{\prime \prime}=0.162$, and $\mu=$ 0.27 . For $x=0.12, t^{\prime}=-0.227, t^{\prime \prime}=0.168$, and $\mu=0.486$. We obtained these values by fitting the tight-binding model by hand, since there was no closed form analytic expression for the tight-binding FS that one could use for least-squares fitting (at 

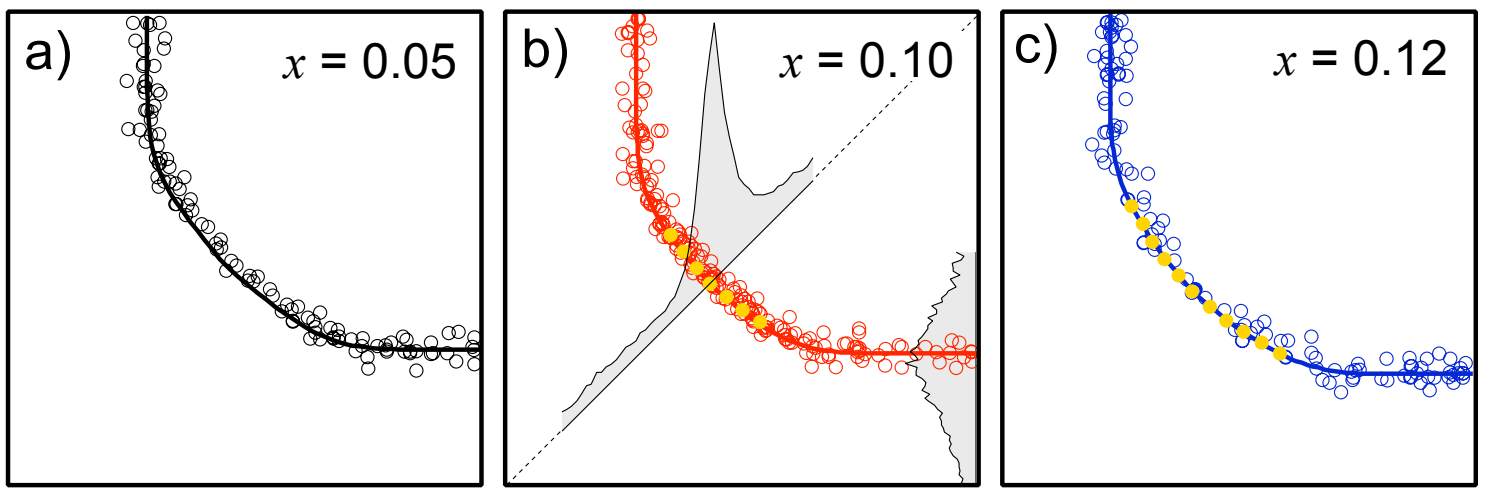

Figure 7.9: Extracted $\mathbf{k}_{\mathrm{F}} \mathrm{s}$ from $\mathrm{Ca}_{2-x} \mathrm{Na}_{x} \mathrm{CuO}_{2} \mathrm{Cl}_{2}$ using the MDC methodology. Data were collected on multiple samples over multiple Brillouin zones, and $\mathbf{k}_{\mathrm{F}} \mathrm{s}$ were translated into the irreducible octent of the 2D square unit cell (open symbols), together with a fit of the data (solid data). Yellow circles show the locations in momentum space along the FS where a near- $E_{\mathrm{F}}$ peak in the EDCs is observed. Data are shown from $x=0.05$ (a), 0.10 (b), and 0.12 (c). Example MDCs along the nodal and antinodal direction are overlaid in grey on (b).

least in two dimensions). Moreover, we did not use the QP dispersion (intensity at $\omega<\mathrm{E}_{\mathrm{F}}$ ) in the tight-binding fits, and therefore these values should be treated simply as a convenient parameterization for the shape of the FS, but not literally as physical values for the electron hopping. The results from the tight-binding fit are shown in Figure 7.10.

\subsection{Charge Ordering in $\mathrm{Ca}_{2-x} \mathrm{Na}_{x} \mathrm{CuO}_{2} \mathrm{Cl}_{2}$}

\subsubsection{Evidence from STM of Charge Ordering}

The primary motivation for the growth of $\mathrm{Ca}_{2-x} \mathrm{Na}_{x} \mathrm{CuO}_{2} \mathrm{Cl}_{2}$ in single-crystalline form was that it would allow a detailed study of the undoped and very lightly doped regime of the cuprate phase diagram by surface sensitive techniques such as ARPES and $\mathrm{STM}$, thanks to its atomically flat $\mathrm{CaCl}$ cleavage planes. Due to materials chemistry constraints, better studied and better known materials such as $\mathrm{Bi}_{2} \mathrm{Sr}_{2} \mathrm{CaCu}_{2} \mathrm{O}_{8+\delta}$ or $\mathrm{YBa}_{2} \mathrm{Cu}_{3} \mathrm{O}_{7-\delta}$ cannot yet be synthesized near the antiferromagnetic Mott insulating 
a)

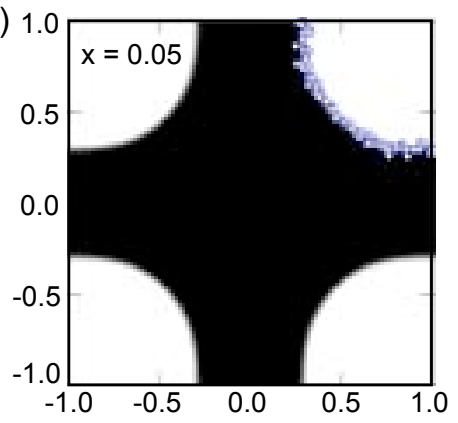

b)

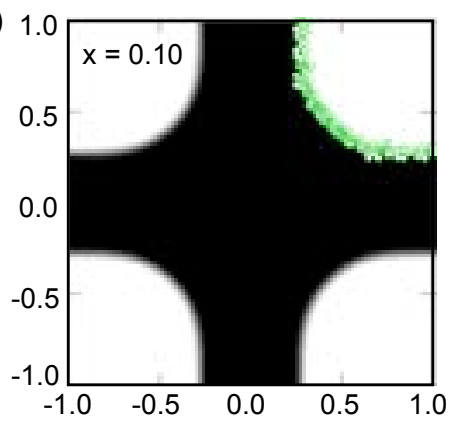

C)

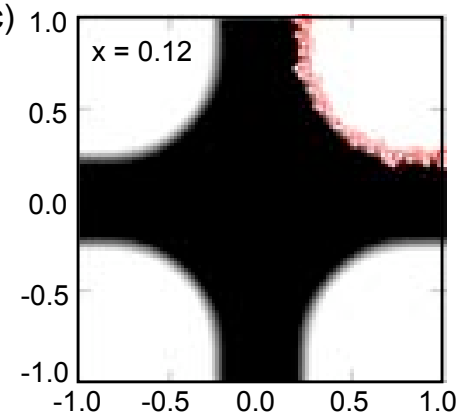

Figure 7.10: Comparison of tight-binding fits of experimental data with tight-binding fits for $x=0.05,0.10,0.12$, as shown in (a), (b), (c). Black areas represent the occupied regions inside the tight-binding FS.

phase. The only other well-known compound that can be chemically synthesized in a clean fashion at such low hole dopings is $\mathrm{La}_{2-x} \mathrm{Sr}_{x} \mathrm{CuO}_{4}$. However, ARPES studies of undoped $\mathrm{La}_{2} \mathrm{CuO}_{4}$ have never produced well-defined and dispersive LHB peaks as observed in the oxychloride compounds, $\mathrm{Ca}_{2} \mathrm{CuO}_{2} \mathrm{Cl}_{2}$ and $\mathrm{Sr}_{2} \mathrm{CuO}_{2} \mathrm{Cl}_{2}$. Moreover, the $\mathrm{La}_{2-x} \mathrm{Sr}_{x} \mathrm{CuO}_{4}$ surface has never yielded well-defined atomically resolved STM images. Therefore, no comparisons have ever been made between the ARPES and STM spectra in the lightly doped regime of the cuprates, until the successful synthesis of $\mathrm{Ca}_{2-x} \mathrm{Na}_{x} \mathrm{CuO}_{2} \mathrm{Cl}_{2}$.

The first STM measurements of $\mathrm{Ca}_{2-x} \mathrm{Na}_{x} \mathrm{CuO}_{2} \mathrm{Cl}_{2}$ were performed by Yuhki Kohsaka and Tetsuo Hanaguri at the University of Tokyo. At that time, detailed STS measurements were not performed, but the constant-current images yielded a striking pattern of nanoscale inhomogeneity. These patterns were determined not to arise from topographic effects, and were therefore strong evidence that significant nanoscale electronic inhomogeneity existed on the surface of $\mathrm{Ca}_{2-x} \mathrm{Na}_{x} \mathrm{CuO}_{2} \mathrm{Cl}_{2}$. These data from [205] are reproduced in Figure 7.11. The STM maps show long, twisting rivers of high conductance (bright) (dubbed as "kishimen", a Japanese noodle!), approximately $4-5 a_{0}$ in width. Autocorrelation maps of the STM images demonstrated anisotropy, in that the kishimen were slightly favored to run along the [100] and [010] crystallographic directions.

More detailed STM/STS measurements were performed in the group of J.C. 
a) $\mathrm{Ca}_{1.92} \mathrm{Na}_{0.08} \mathrm{CuO}_{2} \mathrm{Cl}_{2}$

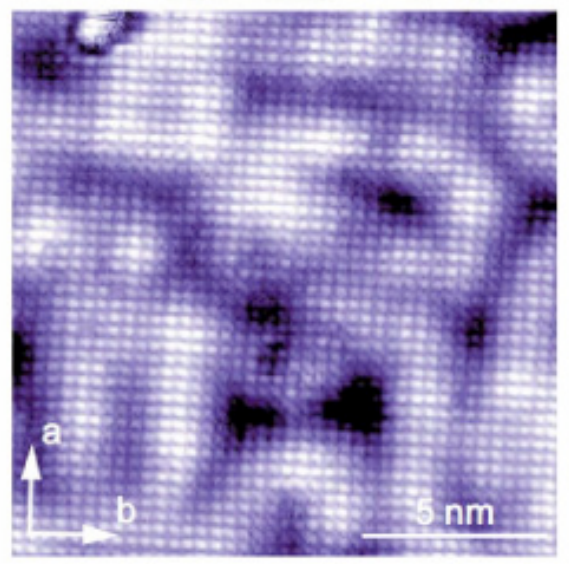

b)

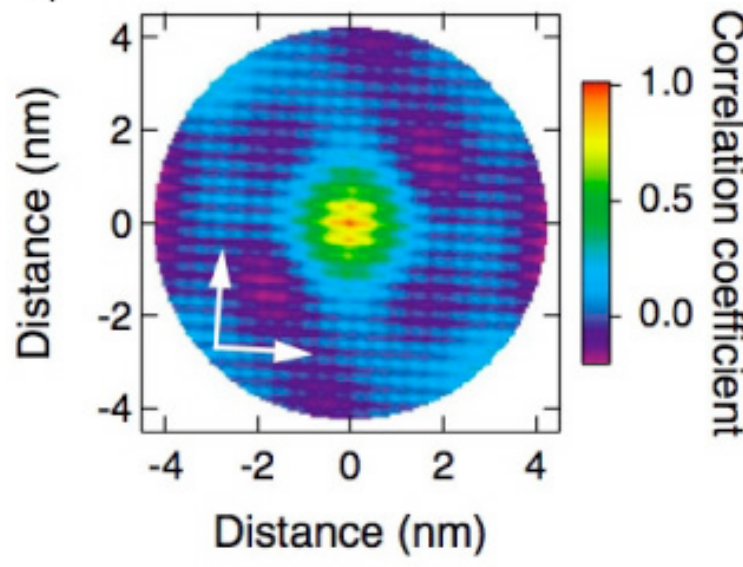

Figure 7.11: (a) $15 \mathrm{~nm} \times 15 \mathrm{~nm}$ STM images of $\mathrm{Ca}_{1.92} \mathrm{Na}_{0.08} \mathrm{CuO}_{2} \mathrm{Cl}_{2}$ taken with $V_{s}$ $=200 \mathrm{mV}$ and $I_{t}=10 \mathrm{pA}$ at $7 \mathrm{~K}$. (b) Autocorrelation patterns of data in (a), taken from Kohsaka et al. [205]

Séamus Davis at Cornell University [169]. STS maps were performed with extremely high spatial resolution, and uncovered a striking pattern of 2D charge-ordering (2DCO) hinted at by the earlier kishimen patterns. The STM data reveal a $4 a_{0} \times 4 a_{0}$ pattern in the $d I / d V$ maps, as shown in Figure 7.12, taken from Hanaguri et al. [169]. In addition, this pattern appeared to be approximately nondispersive, in that the wavevector of the $2 \mathrm{DCO}$ did not deviate from the $4 a_{0} \times 4 a_{0}$ pattern, at least within a window of $\pm 50 \mathrm{meV}$. This is in contrast to the quasiparticle interference patterns or Friedel oscillations observed in $\mathrm{Bi}_{2} \mathrm{Sr}_{2} \mathrm{CaCu}_{2} \mathrm{O}_{8+\delta}$ [206, 207]. Furthermore, the $4 a_{0} \times 4 a_{0}$ pattern in $\mathrm{Ca}_{2-x} \mathrm{Na}_{x} \mathrm{CuO}_{2} \mathrm{Cl}_{2}$ is far more distinct and pronounced than the ordering patterns reported in $\mathrm{Bi}_{2} \mathrm{Sr}_{2} \mathrm{CaCu}_{2} \mathrm{O}_{8+\delta}[208,209]$.

\subsubsection{Evidence for Charge Ordering from ARPES}

Because charge-ordered states (i.e. charge-density-waves) can arise from Fermi surface instabilities such as nesting, ARPES is an obvious and powerful tool to study these systems. To date, a substantial body of work has been performed on "conventional" CDW systems such as $\mathrm{NbSe}_{2}, \mathrm{NbSe}_{3}, \mathrm{TaSe}_{2}, \mathrm{TiSe}_{2}, \mathrm{In} / \mathrm{Cu}(001), \mathrm{Sn} / \mathrm{Ge}(111), \mathrm{CeTe}_{3}$, $\left(\mathrm{TaSe}_{4}\right)_{2} \mathrm{I}$, and $\mathrm{K}_{0.3} \mathrm{MoO}_{3}[210,211,156,157,212,213,214,215,216,217,218]$, to 
a)

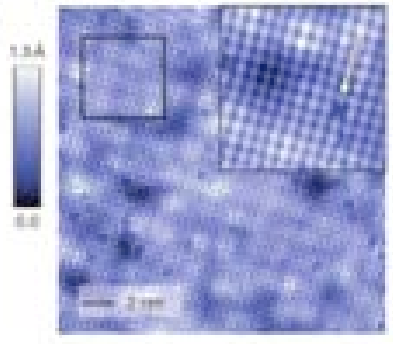

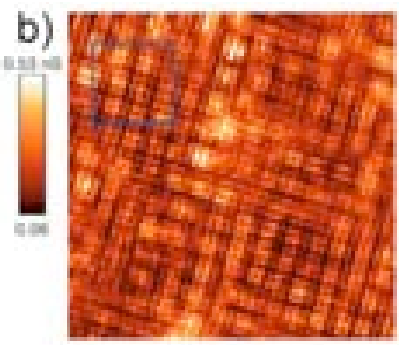

Line cuts of Fourier transtorms
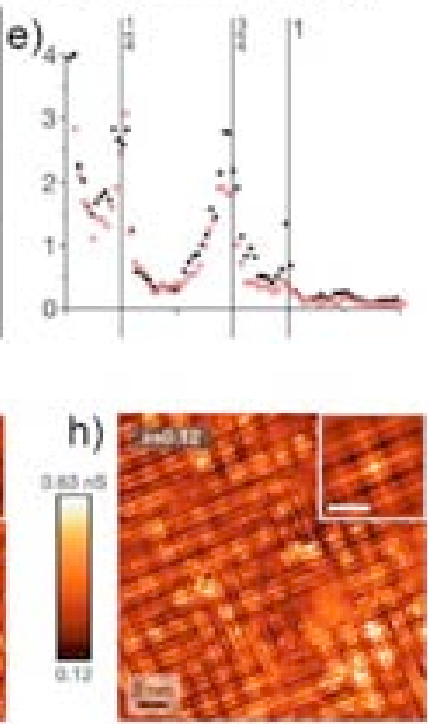

Figure 7.12: (a) STM topographs of $\mathrm{Ca}_{1.9} \mathrm{Na}_{0.10} \mathrm{CuO}_{2} \mathrm{Cl}_{2}$, showing atomic resolution. (b) $d I / d V$ map of same field of view at $E=+24 \mathrm{meV}$, showing the clear 2DCO $4 a_{0} \times 4 a_{0}$ structure. (c) $d I / d V$ map of $\mathrm{Ca}_{1.9} \mathrm{Na}_{0.10} \mathrm{CuO}_{2} \mathrm{Cl}_{2}$ taken at $E=+8 \mathrm{meV}$ and resulting Fourier transform in (d). (e) Line cut of Fourier transform in (d) showing the peaks at $1 / 4$ and $3 / 4$, and the Bragg peak. The $4 a_{0} \times 4 a_{0}$ pattern shows no appreciable doping dependence from $x=0.08,0.10$, to 0.12 (f)-(h). Figures taken from Hanaguri et al. [169] 
a)

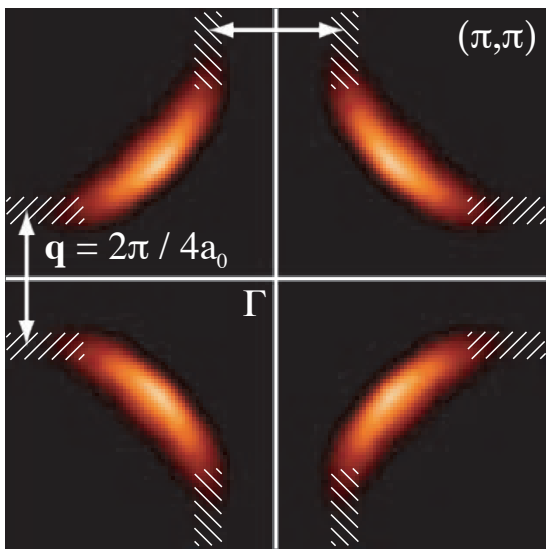

b)

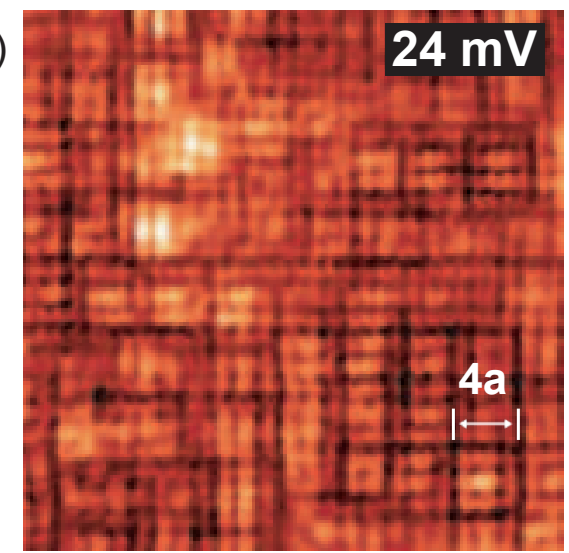

Figure 7.13: (a) Spectral intensity for $\mathrm{Ca}_{1.9} \mathrm{Na}_{0.10} \mathrm{CuO}_{2} \mathrm{Cl}_{2}$ with approximately nested antinodal segments illustrated. (b) $4 a_{0} \times 4 a_{0}$ 2DCO from STM [169].

name a few. In addition, FS nesting and charge-ordering have been reported in the cuprate superconductors [219, 220], although the experimental situations for both $\mathrm{La}_{2-x} \mathrm{Sr}_{x} \mathrm{CuO}_{4}$ and $\mathrm{Bi}_{2} \mathrm{Sr}_{2} \mathrm{CaCu}_{2} \mathrm{O}_{8+\delta}$ have changed considerably in recent years.

On the other hand, $\mathrm{Ca}_{2-x} \mathrm{Na}_{x} \mathrm{CuO}_{2} \mathrm{Cl}_{2}$ provides a much clearer examples, since both STM and ARPES can be performed at very low doping levels. An obvious question is whether one can relate the images from STM/STS to the spectral features observed in ARPES. The $4 a_{0} \times 4 a_{0}$ patterns oriented along the $\mathrm{Cu}$-O bond direction immediately suggests a q-vector that spans across the antinodal Fermi surface neck. By studying Figure 7.9, we can see that the wavevector that connects the antinodal FS segments corresponds very closely to $4 a_{0}$. However, although the q-vector from the ARPES FSes appears to match the $4 a_{0} \times 4 a_{0}$ pattern in the STM/STS images, there is very little spectral weight at $(\pi, 0)$ and $(0, \pi)$. If we used a more conventional quasiparticle scattering picture (essentially a non-interacting, band-like scenario analogous to the QP scattering model $[206,207])$ where we took the autocorrelation image of the the spectral maps shown in Figure 7.3, we would have essentially no intensity at $\mathbf{q}=\left(2 \pi / 4 a_{0}, 0\right)$ due to the lack of spectral intensity at the antinodes. Therefore, there appears to be a rather intriguing dichotomy between the case of STM, where the ostensibly antinodal $4 a_{0} \times 4 a_{0}$ ordering is dominant, and ARPES, where the nodal quasiparticles are far more pronounced than the weak antinodal segments 

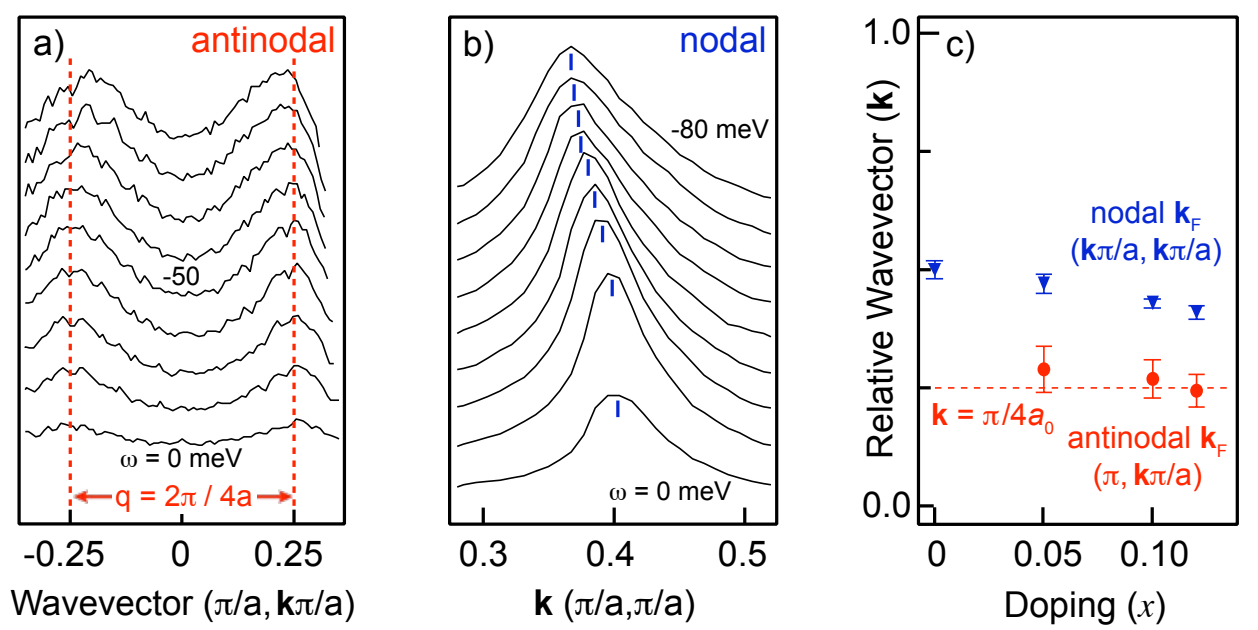

Figure 7.14: (a) Antinodal MDCs from $(\pi,-\pi)-(\pi,+\pi)$ as a function of binding energy every $10 \mathrm{meV}$ from $\mathrm{E}_{\mathrm{F}}$. (b) Nodal MDCs along the $(0,0)-(\pi, \pi)$ direction. (c) Doping dependence of the the nodal and antinodal $\mathbf{k}_{\mathrm{F}} \mathrm{s}$.

which are associated with charge ordering. In Figure 7.13, we show a schematic of the nested antinodal FS segments evidenced by ARPES and their correspondence to the $4 a_{0} \times 4 a_{0}$ checkerboard observed by STM. One picture that has been proposed to explain the observed 2DCO as a Wigner crystal driven by the long-range Coulomb repulsion of the doped holes, and may provide an appealing real-space visualization [221]. However, for the simple Wigner crystal picture, virtually all the holes should be locked into an insulating superlattice and the low-energy QPs would arise from the overflow or deficit of holes away from favored commensurate dopings. This simple picture would be rather difficult to reconcile with our observation of nodal QPS whose intensity appears to increase monotonically with doping. Furthermore, it is not altogether clear how naturally one can obtain nodal QPs in one-dimensional stripe models, although it has been argued that they may arise from disordered stripes [200], but then may only be peripheral states.

In addition to the correspondence of the wavevectors, the energy dependence of both the $4 a_{0} \times 4 a_{0}$ pattern and the antinodal states also share a close resemblance. This is shown in Figure 7.14a, where MDCs along the antinodal direction are plotted 
as a function of binding energy. While the antinodal excitations are broad in momentum space, the peak position of the MDCs shows very weak dependence on $\omega$, which would correspond to a very steep dispersion. This is in contrast to the nodal MDCs shown in Figure 7.14b, where the nodal peaks are much more sharply defined in kspace and exhibit considerable dispersion. This dichotomy between the nodal and antinodal dispersion is particularly unusual given the fact that band theory predictions give the bottom of the band at $(\pi, 0)(\sim-0.25 \mathrm{eV})$ is predicted to be $\sim 8$ times below that of the bottom of the band at $(0,0)(\sim-2 \mathrm{eV})$, and therefore the band velocity along the nodal direction should be even larger than the antinodal direction, in contrast to what is observed experimentally. The doping dependent values of the antinodal and nodal $\mathbf{k}_{\mathrm{F}}$ are given in Figure 7.14c.

As mentioned earlier, the values for the antinodal $\mathbf{k}_{\mathrm{F}} \mathrm{S}$ appear to match rather closely with $\mathbf{k}=2 \pi / 4 a_{0}$, shown as the dashed red line, consistent with $4 a_{0} \times 4 a_{0}$ order. The error bars for the antinodal wavevectors are larger, primarily due to the difficulty in accurately determining the MDC peak maximum along the antinodal direction. In addition, this antinodal wavevector appears to be quite weakly doping dependent, especially given that the van Hove singularity at $(\pi, 0)$ is predicted to be rather shallow below $\mathrm{E}_{\mathrm{F}}$, and therefore changes in $\mu$ due to hole doping should result in relatively large changes in the antinodal $\mathbf{k}_{\mathrm{F}}$. However, this would also be consistent with the fact that the $4 a_{0} \times 4 a_{0}$ patterns observed by STM appear to be essentially doping independent, as shown in Figure 7.12. The antinodal $\mathbf{k}_{\mathrm{F}}$ still appears to exhibit some weak dependence on doping, and appears to close as a function of hole concentration. In principle, this could still be consistent with data from Hanaguri et al. [169], if one considers that the slightly incommensurate wavevectors may arise from a macroscopic superposition of microscopically commensurate regions $\left(4 a_{0} \times 4 a_{0}\right)$, and other incommensurate regions or regions with slightly smaller ordering vectors, q, resulting in an average incommensurate wavevector. The images shown in Figure 7.12 do not exhibit long-ranged $4 a_{0} \times 4 a_{0}$ ordering over the entire field of view. Taking this $\mathbf{k}$-space view of the $4 a_{0} \times 4 a_{0}$ order observed in STM, one can then explain the strong peaks seen in the FT-STS at $\mathbf{q}=(3 / 4 \pi, 0)$ in Figure 7.12 as an umklapp process from a section of FS in the first zone to the second zone. Finally, we should 
emphasize that the evidence for static $2 \mathrm{DCO}$ in $\mathrm{Ca}_{2-x} \mathrm{Na}_{x} \mathrm{CuO}_{2} \mathrm{Cl}_{2}$ is only available from surface-sensitive probes such as STM and ARPES. On the one hand, this fact ensures that the comparison between STM and ARPES is valid, since both probes measure effectively the same electronic states. On the other hand, it would be essential to verify that this phenomenon is also present in the bulk, using techniques such as scattering. It could also be that the observed $4 a_{0} \times 4 a_{0}$ ordering is a result of incipient or fluctuating charge ordering tendencies in the bulk, and the perturbation associated with surface termination is enough to pin or lock in the charge ordered state [222], and remains an open issue of great importance and interest.

\subsubsection{Comparison with Other Charge-Ordered Systems Studied by ARPES}

It has long been known that CDW formation and SC are competing instabilities in a wide variety of materials [223]. This is not surprising, given that the same attractive effective interactions, usually electron-phonon, can give rise to both states in many materials. In addition, the identification of similar charge modulation patters by STM in $\mathrm{Bi}_{2} \mathrm{Sr}_{2} \mathrm{CaCu}_{2} \mathrm{O}_{8+\delta}$ also suggests the possible universality of electronic ordering in all cuprates. Electronic checkerboard patterns are evident in $\mathrm{Bi}_{2} \mathrm{Sr}_{2} \mathrm{CaCu}_{2} \mathrm{O}_{8+\delta}$ when $\mathrm{SC}$ is destroyed (above $\mathrm{T}_{\mathrm{c}}$ [208], inside vortex cores [224], and in very underdoped samples [225]), and may be associated with the absence of sharp antinodal SC excitations in many cuprates studied at low doping levels and with low $\mathrm{T}_{\mathrm{c}} \mathrm{s}$, such as $\mathrm{La}_{2-x} \mathrm{Sr}_{x} \mathrm{CuO}_{4}$ and $\mathrm{Ca}_{2-x} \mathrm{Na}_{x} \mathrm{CuO}_{2} \mathrm{Cl}_{2}$, or above $\mathrm{T}_{\mathrm{c}}$ in the underdoped materials. Although many particular details of the 2DCO state may be material dependent, there appears to be a general correspondence between

In the case of $\mathrm{Ca}_{2-x} \mathrm{Na}_{x} \mathrm{CuO}_{2} \mathrm{Cl}_{2}$, both $d$-wave $\mathrm{SC}$ and $2 \mathrm{DCO}$ appear to compete for the antinodes, and the strength of one order parameter may come at the expense of the other. For instance, although $\mathrm{Ca}_{2-x} \mathrm{Na}_{x} \mathrm{CuO}_{2} \mathrm{Cl}_{2}$ is a rather poor high- $\mathrm{T}_{\mathrm{c}}$ superconductor, it exhibits very prominent modulations in the STM $d I / d V$ maps. On the other hand, $\mathrm{Bi}_{2} \mathrm{Sr}_{2} \mathrm{CaCu}_{2} \mathrm{O}_{8+\delta}$ is one of the better high- $\mathrm{T}_{\mathrm{c}} \mathrm{SCs}$ (maximum $\mathrm{T}_{\mathrm{c}}=96$ $\mathrm{K})$, but exhibits far less pronounced charge density modulations at low energies [206, 
225, 208]. Along these lines, it may be possible that critical fluctuations between the charge-ordered state and another state (such as $d$-SC) could result in the incoherent antinodal states [226], although in this case, it is not entirely clear whether the nodal QPs would also survive. A related possibility is that the 2DCO does not represent a CDW of single holes, but instead represents a density wave of preformed $d$-wave Cooper pairs, or a pair-density wave (PDW) [227].

At this point, we should make a connection between the charge ordering observed in $\mathrm{Ca}_{2-x} \mathrm{Na}_{x} \mathrm{CuO}_{2} \mathrm{Cl}_{2}$ and other more conventional charge-density wave systems [228]. In the conventional CDW picture, a Peierls instability arises when FS nesting leads to a divergence of the Lindhard susceptibility $\chi_{\mathbf{q}}$ at the nesting wavevector, $\mathbf{q}_{C D W}$. Due to this divergent susceptibility, perturbations in the charge density arising from phonons at a wavevector $\mathbf{q}_{C D W}$, are strongly amplified, and act to screen the interionic restoring Coulomb forces. This leads to a softening of the phonon branch at $\mathbf{q}_{C D W}$, otherwise known as a Kohn anomaly. When the softening of the phonon reaches $\omega=0$, a static lattice distortion becomes frozen in at $\mathbf{q}_{C D W}$, leading to the CDW or Peierls state [228].

In the CDW state, there are now two competing periodicities, the original lattice wavevector and $\mathbf{q}_{C D W}$. In the general incommensurate case, this means that the crystal no longer possesses a long range translational symmetry, since a simple translation operation can never bring the lattice back into itself. In the conventional weak-coupling CDW picture, even in the regions of the FS gapped by the CDW formation (i.e. the regions nested by $\mathbf{q}_{C D W}$ ), QP excitations still exist. This is analogous to Bogoliubov quasiparticle excitations in the gapped regions of a superconductor, where well-defined single-particle excitations exist, but are now of a mixed particlehole character. In the CDW case, the quasiparticles are instead of mixed particleparticle character (i.e. electrons from opposite sides of the nested FS), but sharp single-electron excitations still are present. This certainly appears to be the case in materials such as $\mathrm{CeTe}_{3}$ [218] on $\mathrm{In} / \mathrm{Cu}(001)$ [217]. In contrast, in $\mathrm{Ca}_{2-x} \mathrm{Na}_{x} \mathrm{CuO}_{2} \mathrm{Cl}_{2}$, there are no well-defined QP states at the antinodal zone face, raising the question of how the apparently incoherent states $(Z \rightarrow 0)$ can give rise to the 2DCO observed by STM. In the simple FS nesting picture, one starts from a non-interacting 
band picture to calculate the charge (two-particle) susceptibility, $\chi$. However, it is difficult to generalize between single-particle excitations and two-particle susceptibilities, as these two properties only have a straightforward correspondence in the non-interacting picture.

In contrast to $\mathrm{CeTe}_{3}$, some other CDW systems apparently do not exhibit welldefined QPs in the nested regions. For instance, recent work on the classic 1D Peierls systems suggests that a combination of small polaron formation and a Peierls instability, arising from strong electron-phonon coupling, is responsible for the apparent pseudogap and the spectral features observed by ARPES $[156,157]$. Very similar behavior was also observed in the colossal magnetoresistive compound $\mathrm{La}_{1.2} \mathrm{Sr}_{1.8} \mathrm{Mn}_{2} \mathrm{O}_{7}$ by Chuang et al. [150]. In this case, the broad antinodal features tie in neatly to the discussion of small polaron physics in Chapter 5. As shown in Figure 5.10, the effective electron-phonon coupling appears to be significantly larger at $(\pi, 0)$ than at $(\pi / 2, \pi / 2)$. If we extended this strongly anisotropic coupling to the doped case, we might expect to find that the antinodal excitations are much more strongly coupled to the bosonic field, while the nodal excitations are more weakly coupled, resulting in a situation where $Z_{\text {nodal }} \gg Z_{\text {anti }}$, possibly consistent with our experimental observations. In this case, the antinodal states already have strong electron-phonon coupling inherently built into the undoped insulator, potentially favoring a CDW-like state, and the nesting simply locks in and stabilizes the static charge-ordered state.

\subsubsection{Luttinger Volumes in $\mathrm{Ca}_{2-x} \mathrm{Na}_{x} \mathrm{CuO}_{2} \mathrm{Cl}_{2}$}

Although we have colloquially referred to the contours of near- $E_{\mathrm{F}}$ spectral weight as "Fermi surfaces" (FS), this would imply that well-defined, quasiparticle-like $(Z>0)$ excitations exist all across this contour. This would have definite connotations as to certain properties of the FS. For instance, Luttinger's theorem (also known as the Luttinger sum rule [16]) states that the volume enclosed by the FS should be independent of the strength of electron-electron interactions. Depending on how the FS is defined, the Luttinger theorem can be even more robust than Fermi liquid theory, as discussed for the 1D Luttinger liquids. Moreover, ARPES has been demonstrated to 

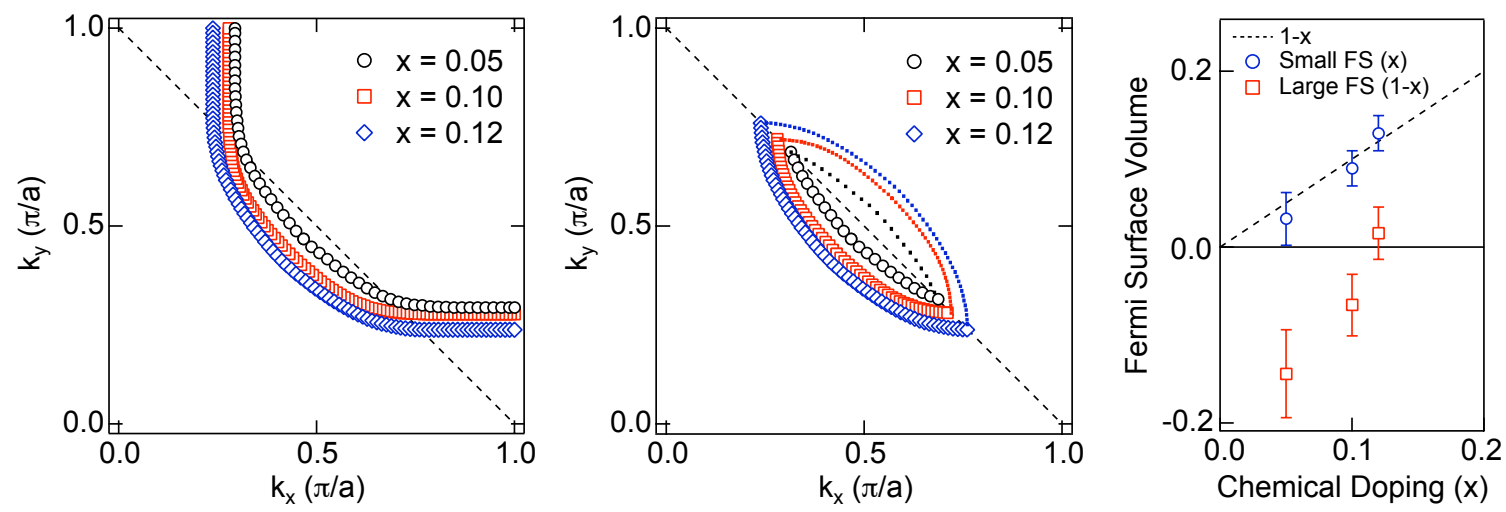

Figure 7.15: (a) Fitted contours to the experimentally determined FSes for $x=0.05$, $0.10,0.12$. (b) Hole pocket centered at $(\pi / 2, \pi / 2)$ generated by reflecting the FSes in (a) about the AF zone boundary. (c) Comparison of experimentally determined Luttinger volumes versus the carrier concentration expected from chemical doping, for a large $1-x$ FS (red squares) and a small $x$ hole pocket (blue circles).

be able to test the Luttinger sum rule quite stringently, as demonstrated for $\mathrm{Sr}_{2} \mathrm{RuO}_{4}$. Therefore, it is of great interest whether or not the lightly doped cuprates near the Mott insulating state obey the Luttinger sum rule. There is the issue of whether one should count " $1-x$ " (taking the view that doping the Mott insulator results in a case similar to doping a band insulator) or " $x$ " (strong correlations, doping rigidly into the LHB), or if neither viewpoint is sufficient.

Using the ostensible FSes described above, we have calculated the Luttinger volume enclosed within these contours by fitting the experimental $\mathbf{k}_{\mathrm{F}} \mathrm{s}$ and calculating the area enclosed in two ways. The first is simply to calculate the entire FS volume $(1-x)$, as shown in Figure 7.15a. The second is to assume that the correct Brillouin zone is the antiferromagnetic Brillouin zone (which is $1 / \sqrt{2} \times 1 / \sqrt{2}$ the size of the crystallographic BZ) and reflect the FS across the AF zone boundary. This would result in a small hole pocket centered around $(\pi / 2, \pi / 2)$, and we neglect the two electron pockets centered around $(\pi, 0)$ and $(0, \pi)$, assuming that the low-lying excitations emerge as one expect from Figure 7.1b, and that we count as $x$. This is shown in Figure 7.15b. The extracted Luttinger volumes are plotted against the expected carrier concentration from the chemical composition (the Na-for-Ca substitution, $x$ ). 
If one assumes a $1-x$ FS, then one obtains the points in red squares in Figure 7.15c; correspondence to the expected Luttinger sum rule for non-interacting electrons is shown as the dashed line. On the other hand, if one counted from $x$, the situation shown in Figure 7.15b, then one would obtain the blue circles shown in Figure 7.15c. This scenario would be consistent with a FS of volume $x$, i.e. doping rigidly into the LHB, while keeping the UHB entirely unoccupied, and seems to agree very well with the expected volume of the hole pocket (dashed line) from the chemical composition. We also note that the values of the area of the small $x$ hole pocket, assuming $x$ carriers / hole will also correspond to a $1-x$ FS, assuming that the starting FS is the AF "diamond" (running from $(0, \pi)$ to $(\pi, 0)$ ), and that the Luttinger volume of this diamond is simply reduced by the nodal arc "biting" a section out of the diamond. Taking this FS (essentially replacing the antinodal straight sections with the AF zone boundary), one can count from $1-x$ and also obtain the blue circles. This type of scenario was described by Furukawa, Rice, and Salmhofer [197] in the context of a 2D Fermi liquid with saddlepoints at $(\pi, 0)$ and $(0, \pi)$, leading to four disconnected "Fermi arcs", ending where the FS intersected the AF zone boundary.

The fact that the apparent FS extracted by the MDC method does not agree with the expectations from Luttinger's sum rule suggests that either this material is non-Fermi liquid-like, or that the observable spectral weight does not provide a good measure of the true underlying FS. From our work, we would conclude that the Luttinger volumes (taking the $1-x$ approach) would correspond to $V_{A R P E S}=$ $-0.14,-0.07$, and 0.02 for the $x=0.05,0.10$, and 0.12 samples, respectively. In fact, simply by measuring the nodal and antinodal $\mathrm{k}_{\mathrm{F}} \mathrm{S}$, assuming the correct symmetry for a square Brillouin zone, and assuming a relatively smooth contour between the node and antinode, one can confirm that Luttinger's sum rule is violated. This is shown below in Figure 7.16, where for $x=0.10$, the enclosed grey area corresponds to a doping of $V=-0.05$ (i.e. 0.05 electron doped). Therefore, if we count from $1-x$, it appears that we badly violate the Luttinger sum rule, making a simple Fermi liquid interpretation problematic. On the other hand, this might imply that while $Z$ might be finite in the nodal arc, it may drop abruptly to zero outside the arc region (for instance, potentially where a QP peak is not seen along the FS, i.e. in 


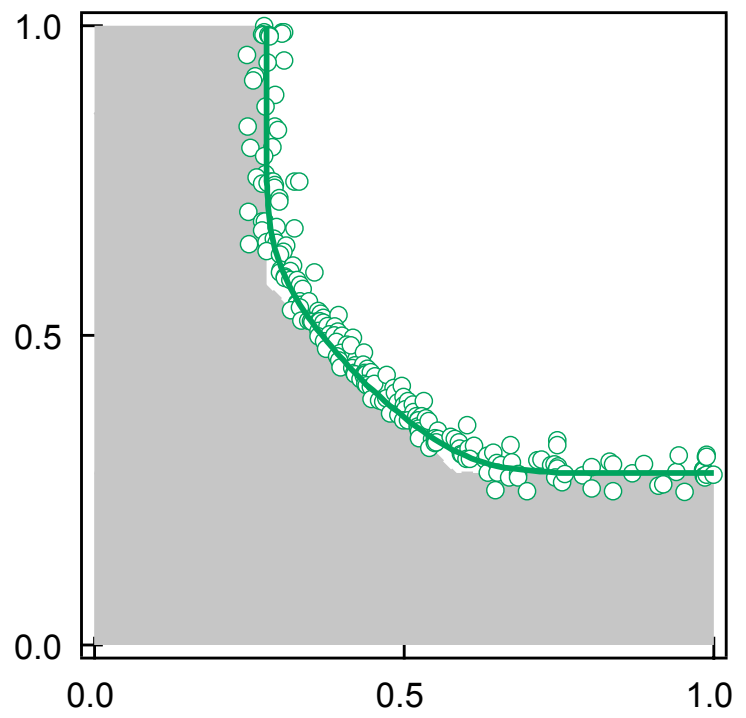

Figure 7.16: Extracted FS for $\mathrm{Ca}_{1.9} \mathrm{Na}_{0.10} \mathrm{CuO}_{2} \mathrm{Cl}_{2}$ and the minimal $1-x$ Luttinger volume area obtained by using straight lines (tangent to the nodal and antinodal contours), shown in grey, giving a $V_{\min }=-0.05$ (electron doped)

Figure 7.9 outside the region with the yellow circles), as suggested in the "hot spot" theories advanced by Rice and Furukawa. If $Z=0$ in the antinodal regions, the Luttinger sum rule can potentially be violated in this system. Although one might expect non-Fermi liquid behavior near the Mott insulating state, it is rather strange to have $Z$ finite in certain regions in $\mathbf{k}$-space, and $Z=0$ in other regions in $\mathbf{k}$-space. Typically, Fermi liquid theory should be an "all-or-nothing" proposition, where $Z$ would be finite everywhere or zero everywhere. Although our previous analysis in Figure 7.5 might imply that $Z$ varies continously across the FS, we cannot rule out the possibility that at some (possibly doping-dependent) $\theta, Z$ drops abruptly to zero (perhaps where a well-defined peak in the EDCs disappears), but the spectral weight at $\mathrm{E}_{\mathrm{F}}$ remains finite.

Along these lines, a recent theoretical work based on dynamical mean-field theory has attempted to calculated $\mathcal{A}(\mathbf{k}, \omega=0)$, or in other words, our low-lying intensity maps. Recent work by Civelli et al. appears to suggest that a situation strikingly similar to our experiments [229]. Specifically, at low dopings, the antinodes have 

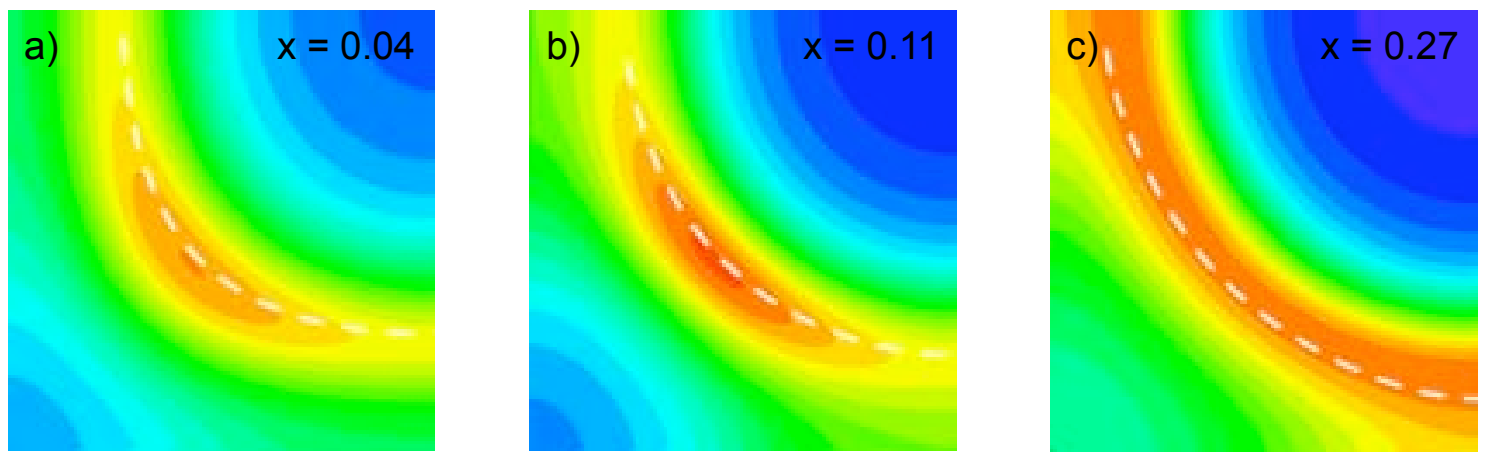

Figure 7.17: Spectral maps for $\mathcal{A}(\mathbf{k}, \omega=0)$ for $x=0.04,0.11$, and 0.27 from Civelli et al. [229].

very weak spectral weight, and moreover, the contours of maximum intensity do not appear to obey the Luttinger sum rule for the calculated hole concentrations. Civelli et al. interpret this as a dynamical breakup of the Fermi surface in "cold" sections (nodal) and "hot" sections (antinodal). Analyzing the contours from [229] shown in Figure 7.17 by taking the MDC maximum as the value for $\mathbf{k}_{\mathrm{F}}$, we would obtain a volume of $V_{D M F T}=-0.04,0.01$, and 0.10 for the $x=0.04,0.11$, and 0.27 calculations shown, demonstrating that the volumes extracted from ARPES and the effective volumes extracted in a similar fashion from DMFT behave in a very similar fashion in terms of underestimating the hole concentration. Therefore, the apparent violation of Luttinger's theoren may arise due to the strong electron correlation effects inherent to the problem of the doped Mott insulator. This was also pointed out earlier in the work of Maier et al. using a dynamical cluster approximation (DCA) to calculate the ARPES spectra of the Hubbard model [230]. In addition, very recent work using variational cluster perturbation theory have obtained rather similar results to those discussed above [231]. However, the fact that the DMFT technique is based on starting around a Fermi liquid fixed point brings into question how meaningful the DMFT results are when they calculate seemingly non-Fermi liquid behavior.

A similar "violation" of the Luttinger sum rule was also seen in very lightly doped $\mathrm{Bi}_{2} \mathrm{Sr}_{2} \mathrm{CaCu}_{2} \mathrm{O}_{8+\delta}$ [106], although studies of the FS in $\mathrm{Bi}_{2} \mathrm{Sr}_{2} \mathrm{CaCu}_{2} \mathrm{O}_{8+\delta}$ were less comprehensive. On the other hand, very detailed studies of $\mathrm{La}_{2-x} \mathrm{Sr}_{x} \mathrm{CuO}_{4}$ from the 
work of Xingjiang Zhou [232] appear to suggest that the FS of $\mathrm{La}_{2-x} \mathrm{Sr}_{x} \mathrm{CuO}_{4}$ appears to coincide rather closely with the expected Luttinger volume from counting $1-x$. This is shown in Figure 7.18a, where the measured volume as a function of $x$ is plotted. This approximate agreement with the expected $1-x$ volume is highly unlike the situation for $\mathrm{Ca}_{2-x} \mathrm{Na}_{x} \mathrm{CuO}_{2} \mathrm{Cl}_{2}$. This can also be seen in the behavior of the nodal and antinodal $\mathbf{k}_{\mathrm{F}} \mathrm{s}$. Upon doping the first holes into $\mathrm{La}_{2-x} \mathrm{Sr}_{x} \mathrm{CuO}_{4}$, the nodal $\mathbf{k}_{\mathrm{F}}$ appears to "jump" discontinuously away from $(\pi / 2, \pi / 2)$ (the maximum of the LHB) to a value more consistent with expectations from one-electron band theory, as shown in Figure 7.18b. On the other hand, the nodal $\mathbf{k}_{\mathrm{F}}$ for $\mathrm{Ca}_{2-x} \mathrm{Na}_{x} \mathrm{CuO}_{2} \mathrm{Cl}_{2}$ seems to evolve smoothly away from $(\pi / 2, \pi / 2)$ as a function of doping, more consistent with the picture of doping a Mott insulator. In addition, the antinodal $\mathbf{k}_{\mathrm{F}} \mathrm{S}$ for $\mathrm{La}_{2-x} \mathrm{Sr}_{x} \mathrm{CuO}_{4}$ appear to be smaller than $\mathrm{Ca}_{2-x} \mathrm{Na}_{x} \mathrm{CuO}_{2} \mathrm{Cl}_{2}$ at any given doping, as shown in Figure 7.18c. At this stage, we cannot ascertain what the origin of this difference is between $\mathrm{Ca}_{2-x} \mathrm{Na}_{x} \mathrm{CuO}_{2} \mathrm{Cl}_{2}$ and $\mathrm{La}_{2-x} \mathrm{Sr}_{x} \mathrm{CuO}_{4}$, although it could potentially be related to the different behaviors of $\mu$ in the two different systems. The pinned behavior of $\mu$ in $\mathrm{La}_{2-x} \mathrm{Sr}_{x} \mathrm{CuO}_{4}$ may suggest the presence of phase separation, in the sense that the system has separated into antiferromagnetic hole-poor regions, and hole-rich regions. Then, the measured FS in $\mathrm{La}_{2-x} \mathrm{Sr}_{x} \mathrm{CuO}_{4}$ might correspond only to the hole-rich regions, therefore accounting for the discrepancy between $\mathrm{Ca}_{2-x} \mathrm{Na}_{x} \mathrm{CuO}_{2} \mathrm{Cl}_{2}$ and $\mathrm{La}_{2-x} \mathrm{Sr}_{x} \mathrm{CuO}_{4}$. On the other hand, one would expect in the case of phase separation a preferred density of holes, whereas in the case of $\mathrm{La}_{2-x} \mathrm{Sr}_{x} \mathrm{CuO}_{4}$, the density of holes would have to change continuously to match $1-x$. In other words, in the case of simple phase separation, one might not expect the Fermi surface wavevectors $\mathbf{k}_{\mathrm{F}} \mathrm{S}$ to change with doping, but simply the overall FS intensity to increase with $x$. However, this intensity increase would be consistent with the observed growth in intensity of the FS, $I_{F S} \propto x$, for both $\mathrm{La}_{2-x} \mathrm{Sr}_{x} \mathrm{CuO}_{4}$ and $\mathrm{Ca}_{2-x} \mathrm{Na}_{x} \mathrm{CuO}_{2} \mathrm{Cl}_{2}$.

Another interesting question that remains to be resolved is whether one can relate the nested antinodal FS segments in $\mathrm{La}_{2-x} \mathrm{Sr}_{x} \mathrm{CuO}_{4}$ to the stripe ordering observed in those compounds, and more generally, the nature of the relationship between the ordering observed in $\mathrm{Ca}_{2-x} \mathrm{Na}_{x} \mathrm{CuO}_{2} \mathrm{Cl}_{2}$ and $\mathrm{La}_{2-x} \mathrm{Sr}_{x} \mathrm{CuO}_{4}$. In LSCO, the tendency to possibly form one-dimensional stripes may arise from the orthorhombic structural 

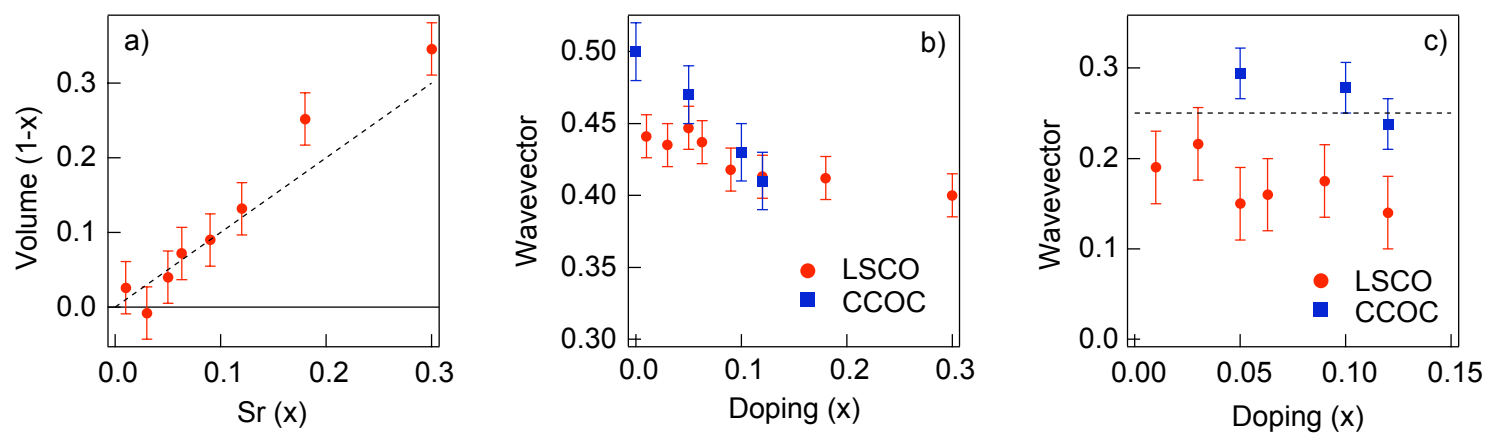

Figure 7.18: (a) Extracted volume from FS mappings of $\mathrm{La}_{2-x} \mathrm{Sr}_{x} \mathrm{CuO}_{4}$ from Zhou et al. [232] versus the nominal Sr concentration, showing approximate agreement of $1-x$ counting. (b) Doping dependence of the nodal $\mathbf{k}_{\mathrm{F}}$ from $\mathrm{Ca}_{2-x} \mathrm{Na}_{x} \mathrm{CuO}_{2} \mathrm{Cl}_{2}$ and $\mathrm{La}_{2-x} \mathrm{Sr}_{x} \mathrm{CuO}_{4}$. (c) Doping dependence of the antinodal $\mathbf{k}_{\mathrm{F}}$.

distortions (LTO-to-LTT) which may pin charge order along certain lines, or "creases" in the lattice. On the other hand, it might be the case that one-dimensional ordering is naturally favored, even in $\mathrm{Ca}_{2-x} \mathrm{Na}_{x} \mathrm{CuO}_{2} \mathrm{Cl}_{2}$. At this point the correlation lengths observed in STM experiment are short enough that some directional anisotropy might exist over short length scales, but over large fields of view, the $a / b$ anisotropy becomes completely averaged out. Therefore, one needs to more carefully examine the STM images for short-ranged anisotropy. Certainly in our ARPES experiments, one averages over $\sim$ mm-sized areas, and thus we obtain a spatial average, thereby leaving open the possibility for one-dimensional anisotropy.

At present, a promising potential avenue to explain the spectral features in the undoped and lightly doped Mott insulator may be an approach based on DMFT + phonons. On one level, small polaron physics and strong electron-phonon coupling appears to explain very nicely many of the spectral features observed in the undoped insulator, and potentially the strong and anisotropic electron-boson coupling. At another level, the DMFT may well account for additional features beyond the conventional small polaron model due to strong electronic correlations, such as the Hubbard gap, the very strongly temperature dependent LHB width, and the apparent violation of the Luttinger sum rule. However, the computational power needed to 
perform such calculations is extremely large, and therefore may prove highly challenging. Nevertheless, such computations may shed crucial insights into the nature of the interplay between strong electron correlations and lattice coupling in the transition metal oxides. 


\section{Chapter 8}

\section{Conclusions and Future Prospects}

\subsection{Conclusions}

In this dissertation, we have presented a new and unified view of the hole doping evolution of the cuprate $\mathrm{Ca}_{2-x} \mathrm{Na}_{x} \mathrm{CuO}_{2} \mathrm{Cl}_{2}$ from an undoped Mott insulator to a high- $\mathrm{T}_{\mathrm{c}}$ superconductor. In the past, our tentative understanding of the undoped and lightly doped regimes meant that issues such as the evolution of the chemical potential, the spectral lineshape, the emergence of quasiparticle states, and the transfer of spectral weight were all understood in a piecemeal and inconsistent fashion. Our work, which introduces a novel approach to understanding the single-particle spectra of the Mott insulator in a consistent fashion, provides for the first time a phenomenological picture in which one can explain the evolution of $\mu$, the doping dependence of the spectral lineshape, the emergence of quasiparticle states, and the doping dependence of the Fermi wavevector $\mathbf{k}_{\mathrm{F}}$ and the Fermi velocity $v_{\mathrm{F}}$. Although this is still a heuristic scenario and does not explain the microscopic details, it still provides a coherent framework for understanding and interpreting the ARPES spectra.

The key advance in this understanding was the reinterpretation of the ARPES spectra from the undoped Mott insulator in terms of a small polaron / Franck-Condon broadening scenario. This meant revising the previous interpretation: instead of the spectral peak in the Mott insulator representing a QP pole, we assign the peak to be 
the maximum in a broad envelope of boson shake-off sidebands, as detailed in Chapters 4 and 5. The true quasiparticle ("0-0" transition) sits far out in the low-energy tail of the broad peak, has nearly vanishing QP weight, and is effectively dispersionless. Recent theoretical work by Mishchenko [141, 173] and Rösch [170] suggests that one can also reconcile the dispersive nature of these broad sidebands and their apparent agreement with theories incorporating strong electron-electron correlations, such as $t-t^{\prime}-t^{\prime \prime}-J$ models. In this sense, one can view the spectra from the undoped Mott insulator as resulting from different interactions and energy scales: the kinetic energy, $t$, the antiferromagnetic exchange energy, $J$, and a low-energy bosonic field (either phonons or magnons). To determine whether the polaronic states arose from electron-magnon or electron-phonon interactions, we performed comparisons in Chapter 5 between the states in the lower Hubbard band, and other electronic states decoupled from the spin system (the $\mathrm{O} 2 p_{\pi}$ and Ca $3 p$ states). This work led us to conclude that a large contribution of observed polaronic broadening did indeed originate from lattice interactions.

Armed with this new understanding of the spectral features and polaron formation in the undoped system, we can now better interpret the doping evolution of the electronic states in $\mathrm{Ca}_{2-x} \mathrm{Na}_{x} \mathrm{CuO}_{2} \mathrm{Cl}_{2}$, as described in Chapter 6. First of all, the assignment of the QP pole in the insulator to the low-energy tail allows to explain why the minimum pinned position of $\mu$ in the insulator was always $\sim 400 \mathrm{meV}$ away from the broad peak maximum. Most importantly, this helps us explain why the chemical potential does not abruptly drop $400 \mathrm{meV}$ to the spectral peak maximum immediately upon doping the first hole into the system. Using our polaronic interpretation in concert with high-resolution measurements of the $\mathrm{O} 2 p$ states, we find that $\mu$ shifts smoothly and rapidly into the lower Hubbard band. In addition, we find that the changes in the Fermi wavevector $\mathbf{k}_{\mathrm{F}}$ and $\mu$ with doping are related simply through $v_{\mathrm{F}}$, suggesting almost a "rigid band" shift into the faint, low-energy quasiparticle branch. However, the real scenario is not as straightforward as a simple rigid band shift, as strong spectral weight transfer occurs at all energy scales.

Finally, we investigate the momentum dependence of the first doped holes in $\mathrm{Ca}_{2-x} \mathrm{Na}_{x} \mathrm{CuO}_{2} \mathrm{Cl}_{2}$ throughout the Brillouin zone in Chapter 7. Our work establishes 
that in the underdoped regime, the nodal quasiparticle excitations are dominant, while the antinodal excitations are very faint, resulting in an arc-like distribution of spectral weight. The overall intensity of this Fermi arc appears to grow roughly monotonically with hole doping, in a manner commensurate to our discussion of spectral weight transfer in Chapter 6. In both these aspects, this general scenario appears qualitatively similar to the situation observed in lightly doped $\mathrm{La}_{2-x} \mathrm{Sr}_{x} \mathrm{CuO}_{4}$ $[171,233]$. Despite the faint spectral weight in the antinodal regions, they exhibit a nesting wavevector $\mathbf{q}=2 \pi / 4 a_{0}$ which appears to correspond very closely to the $4 a_{0} \times$ $4 a_{0}$ checkerboard charge-ordering observed by STM, indicating that the electronic states comprising the $4 a_{0} \times 4 a_{0}$ checkerboard originate from the antinodal states. However, this should not be confused with a case of simple, weak-coupling Fermi surface nesting where quasiparticle excitations still remain well-defined even in the gapped regions, much like Bogoliubov quasiparticles in a superconductor. However, we find only indistinct, seemingly incoherent excitations in the antinodal regions. Relating this back to our work on Franck-Condon broadening in Chapter 5, this could correspond to antinodal states which are more strongly coupled to a bosonic (likely phonons) field, therefore resulting in the broad excitations.

\subsection{Future Directions}

Regarding our work on polaron formation and Franck-Condon broadening in the cuprates, one obvious question to determine precisely how much of a contribution does the lattice introduce, how much arises from antiferromagnetism, and how much is simply due to strong electron correlations. Our work in Chapter 5 provided an important first step in indicating that lattice effects comprised a substantial amount of the observed Franck-Condon broadening, but the lattice effects on the LHB were still only inferred. A true "smoking gun" experiment would be to observe a clear isotope effect (say, ${ }^{16} \mathrm{O}$ for ${ }^{18} \mathrm{O}$ ) in the width of the lower Hubbard band, as this should result in a different $\Gamma_{F W H M}$ assuming a change in the phonon frequency $\omega_{0}$. This would be even more convincing if one did not observe large changes in the lattice constants, $\mathrm{T}_{N}$, or $J$. However, due to the great chemical stability of the oxychloride 
materials, oxygen isotope exchange is extremely difficult and much more challenging than a similar procedure for $\mathrm{Bi}_{2} \mathrm{Sr}_{2} \mathrm{CaCu}_{2} \mathrm{O}_{8+\delta}$. Nevertheless, this should, in many ways, be the best method for observing lattice effects in the cuprates and establishing or disproving the importance of electron-phonon interactions.

In addition to studying isotope and polaronic effects by ARPES, our interpretation of the Franck-Condon broadened lower Hubbard bands from ARPES has important implications for the value and determination of the charge-transfer gap in. Ignoring excitonic effects, the optical conductivity should be a convolution or joint density of states of the valence and conduction bands at $\mathbf{q}=0$. In conventional one-electron pictures, the main peak in the optical conductivity spectrum should then correspond to transitions between the quasiparticle poles in the valence and conduction bands. However, in a highly polaronic picture, the broad multi-boson sidebands dominate the spectral weight, and actual quasiparticle has vanishingly small weight. Therefore the actual quasiparticle (or polaron) gap in the cuprates could be dramatically reduced from the typical experimentally deduced value of the charge-transfer gap. Again this is a direct consequence of the fundamental difference between the position of the quasiparticle poles and the spectral weight maximum in highly polaronic systems. This should also be observable from an isotope effect and would again be a "smoking gun" for proving or disproving lattice polaron formation. Moreover, such effects in the upper Hubbard band alone might also be observable from x-ray absorption spectroscopy (XAS) and inverse photoemission (although practically speaking, the counting rates and energy resolution for inverse photoemission make this practically infeasible).

Finally, extending the measured doping range of $\mathrm{Ca}_{2-x} \mathrm{Na}_{x} \mathrm{CuO}_{2} \mathrm{Cl}_{2}$ would be of critical importance, if single crystals can be synthesized. For instance, extremely lightly doped samples would be highly desired $(x \sim 0.01)$, to see the evolution of the Franck-Condon sidebands and $\mu$ at very low dopings, as currently the gap between $x=0$ and the first $x>0$ composition is rather sizable $(\Delta x=0.05)$. Perhaps more importantly, an investigation of the optimally and overdoped compositions would be extremely valuable to contrast against the undoped and lightly doped materials. For instance, in $\mathrm{La}_{2-x} \mathrm{Sr}_{x} \mathrm{CuO}_{4}$, it has been observed that the anisotropy in the angular 
distribution of low-energy spectral weight "flip-flops" very abruptly from being maximal along the nodal direction in the underdoped samples to being maximal at the antinodes in the overdoped samples. Furthermore, it would be extremely interesting to see whether such a crossover is also seen in $\mathrm{Ca}_{2-x} \mathrm{Na}_{x} \mathrm{CuO}_{2} \mathrm{Cl}_{2}$ and whether this corresponds to the emergence of well-defined antinodal quasiparticles as well as the disappearance of charge-ordering (i.e. the competing order parameter). 


\section{Appendix A}

\section{Fully Gapped Excitations in the Lightly Doped Cuprates}

\section{A.1 Background}

While accurately describing the single particle excitations of the undoped insulator remains a theoretical challenge, this problem becomes far more daunting upon the addition of even a small number of holes or electrons ${ }^{1}$. The exotic properties exhibited by these underdoped cuprates have led to numerous inquiries and debates over the physics of the insulator-superconductor transition, the presence of competing phases, precursor superconductivity, and electronic phase separation. While popular theoretical models (i.e. $t-J$ or Hubbard models) predict the formation of metallic states even at infinitesimally small doping concentrations [140], antiferromagnetic Néel order has been experimentally found to persist up to finite doping levels. Moreover, the doping range in which low temperature insulating behavior is observed has been universally found in the cuprates to extend well past the disappearance of Néel order [234]. This naturally raises the question of the respective roles played by order (i.e. Néel order or alternative competing orders) and disorder (chemical or electronic inhomogeneity), on the low lying electronic states derived from doping the parent insulator.

\footnotetext{
${ }^{1}$ The vast majority of the work discussed in this chapter has been published in K.M. Shen et al., Physical Review B 69, 054503 (2004).
} 
To date, angle-resolved photoemission spectroscopy (ARPES) has been the premier tool for the direct study of the electronic structure of the near-optimally doped cuprates [40]. However, its contributions to our understanding of the lightly doped regime have been extremely limited. The main reason for this disparity is that the bismuth based cuprates, the archetypal materials for ARPES, are naturally grown within a limited range around optimal doping, and thus it becomes necessary to study other families in order to access lighter dopings. Because of the extreme dearth of ARPES data in the lightly doped regime, a serious gap exists in our experimental understanding of the doping evolution of the electronic structure. Unfortunately, this deficiency in knowledge occurs where the physics of the cuprates is generally acknowledged to be most complex, further complicating attempts at understanding the physics of high-temperature superconductivity. It has been very recently shown that upon the addition of even a small concentration of carriers to the parent insulator, finite spectral weight develops near the chemical potential [26, 171], as expected for a compressible thermodynamic system. While consistent with the high temperature metallic behavior seen at low concentrations $[234,36]$, this near- $E_{F}$ weight has serious conflicts with the low temperature insulating behavior observed by charge and thermal transport $[234,36,235,236]$. It is therefore important to ask whether this near- $\mathrm{E}_{\mathrm{F}}$ weight is additionally gapped at low energies. An energy gap along the $d$ wave node has not yet been observed, as previous work in the pseudogap regime was restricted to higher dopings where the nodal states were found to be ungapped. The presence of a gap along the nodal direction will clearly demonstrate the effects of disorder or additional orders on the ostensibly $d$-wave-like low energy states.

Here we report an extensive ARPES study of various lightly doped cuprates, where we find a small but apparently finite excitation gap in the normal state over the entire Brillouin zone. This result is observed for a variety of compounds and carrier concentrations, including hole-doped $\mathrm{Ca}_{2-x} \mathrm{Na}_{x} \mathrm{CuO}_{2} \mathrm{Cl}_{2}(x=0.05,0.10), \mathrm{La}_{2-x} \mathrm{Sr}_{x} \mathrm{CuO}_{4}$ $(x=0.01,0.02)$, and electron-doped $\mathrm{Nd}_{2-x} \mathrm{Ce}_{x} \mathrm{CuO}_{4}(x=0.025,0.04)$. This study reports the lowest doping concentrations ever studied by ARPES for each of the respective compounds, including $\mathrm{La}_{2-x} \mathrm{Sr}_{x} \mathrm{CuO}_{4}$ with $x \leq 0.02$, where Néel order persists. The widespread absence of ungapped excitations in this regime suggests 
that this behavior may be generic to the cuprate superconductors instead of being a material-specific phenomenon. We consider a number of scenarios, including the presence of disorder and electronic inhomogeneity, as well as a possible competing order.

\section{A.2 Experimental Details and Results}

Measurements were performed with both synchrotron radiation and a He discharge lamp with typical energy and angular resolutions of 10 to $14 \mathrm{meV}$ and $0.3^{\circ}$, respectively. Even with an energy resolution of $14 \mathrm{meV}$, edge positions could be measured accurately and reproducibly to within $1 \mathrm{meV}$. The Fermi energy $\left(\mathrm{E}_{\mathrm{F}}\right)$ was determined from a polycrystalline Au target in direct electrical contact with the sample. Single crystals of $\mathrm{La}_{2-x} \mathrm{Sr}_{x} \mathrm{CuO}_{4}$ and $\mathrm{Nd}_{2-x} \mathrm{Ce}_{x} \mathrm{CuO}_{4}$ were grown using the travelling-solvent floating zone method, while single crystals of $\mathrm{Ca}_{2-x} \mathrm{Na}_{x} \mathrm{CuO}_{2} \mathrm{Cl}_{2}$ were grown using a self-flux method [114]. Samples were first aligned by Laue diffraction ex situ, and cleaved and measured at a base temperature of $15 \mathrm{~K}$ at a pressure of better than $5 \times 10^{-11}$ torr.

As previously observed in $\mathrm{La}_{2-x} \mathrm{Sr}_{x} \mathrm{CuO}_{4}$ and $\mathrm{Bi}_{2} \mathrm{Sr}_{2} \mathrm{CaCu}_{2} \mathrm{O}_{8+\delta}[172,171]$, the first hole addition states emerge near $\left(\frac{\pi}{2}, \frac{\pi}{2}\right)$, the top of the lower Hubbard band of the undoped parent insulator. At relatively low concentrations, the locus of low-lying spectral weight is confined to a small arc. Outside this sector, a large pseudogapped region devoid of well-defined low energy excitations persists around $(\pi, 0)$. As an example, we show spectra from $\mathrm{Ca}_{1.9} \mathrm{Na}_{0.1} \mathrm{CuO}_{2} \mathrm{Cl}_{2}$ in Figure A.1 whose electronic structure has been shown to be consistent with the behavior described throughout this thesis. A dispersive excitation branch can be observed along the $(0,0)-(\pi, \pi)$ line in Figure A.1a. In Figure A.1b, all spectra have been collapsed together and there exists a clear shift of all leading edge midpoints away from the chemical potential which we call a leading edge gap (LEG). As mentioned above, the locus of low-lying excitations in this compound is confined to an arc-like segment spanning approximately $\pm 20^{\circ}$ measured radially from $(0,0)$, as discussed in Chapter 7 . The angular dependence of this gap, $\Delta_{\mathrm{LEG}}$, shown in Figure A.1c, exhibits weak anisotropy within this arc. 


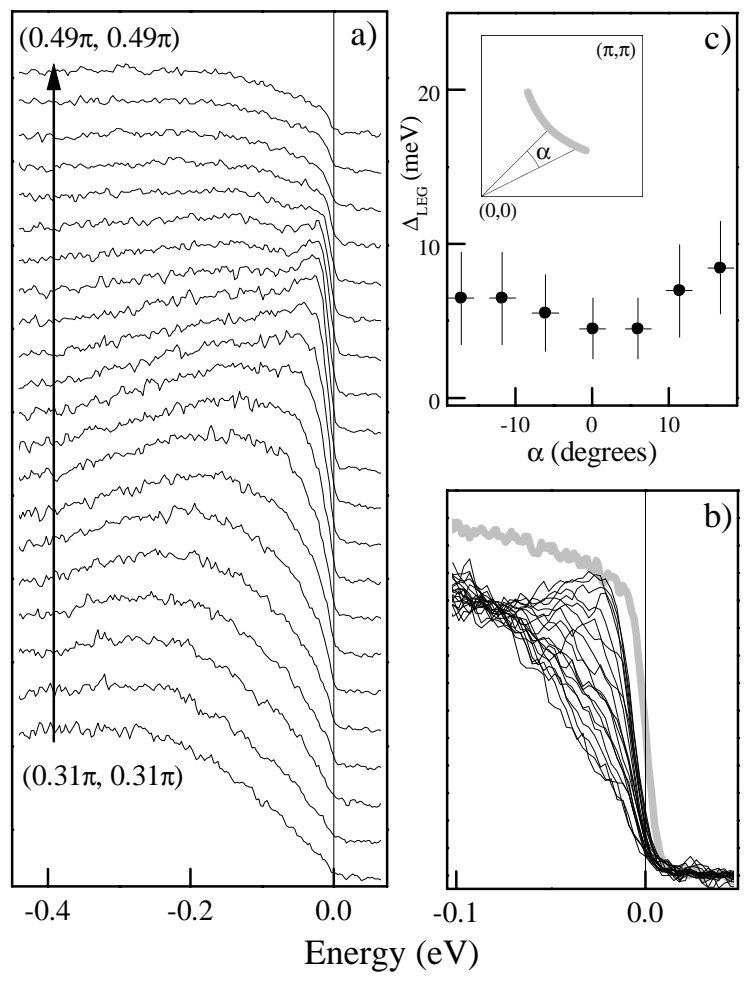

Figure A.1: (a) Spectra from $\mathrm{Ca}_{1.9} \mathrm{Na}_{0.1} \mathrm{CuO}_{2} \mathrm{Cl}_{2}$ taken along $(0,0)$ to $(\pi, \pi)$. (b) Collapsed spectra from (a) with a finite leading edge gap of $5 \mathrm{meV}$ as deduced from comparison with $\mathrm{Au}$ (grey). (c) Momentum dependence of this leading edge gap along the low-energy arc as a function of angle $\alpha$.

However, it is difficult to ascertain whether this apparent anisotropy is intrinsic, or due to the loss of spectral weight and broader lineshapes away from the nodal line, as illustrated by the larger error bars.

Spectra from non-superconducting $(x=0.05)$ and underdoped $\left(x=0.10, \mathrm{~T}_{\mathrm{c}}=\right.$ $\left.13 \mathrm{~K} ; x=0.12, \mathrm{~T}_{\mathrm{c}}=22 \mathrm{~K}\right)$ compositions of $\mathrm{Ca}_{2-x} \mathrm{Na}_{x} \mathrm{CuO}_{2} \mathrm{Cl}_{2}$ are shown at the bottom of Figure A.2. While no well-defined peak is visible for $x=0.05$, there exists a distinct edge structure with $\Delta_{\mathrm{LEG}}=7 \mathrm{meV}$. This effect decreases with hole doping and appears to close by $x=0.12$. To demonstrate that this effect is generic to all cuprates, we also present results from very lightly doped, non-superconducting $\mathrm{La}_{2-x} \mathrm{Sr}_{x} \mathrm{CuO}_{4}$ and $\mathrm{Nd}_{2-x} \mathrm{Ce}_{x} \mathrm{CuO}_{4}$ in Figure A.2, summarizing our findings regarding this LEG. For $\mathrm{La}_{2-x} \mathrm{Sr}_{x} \mathrm{CuO}_{4}$, the topology of low-lying excitations is qualitatively 


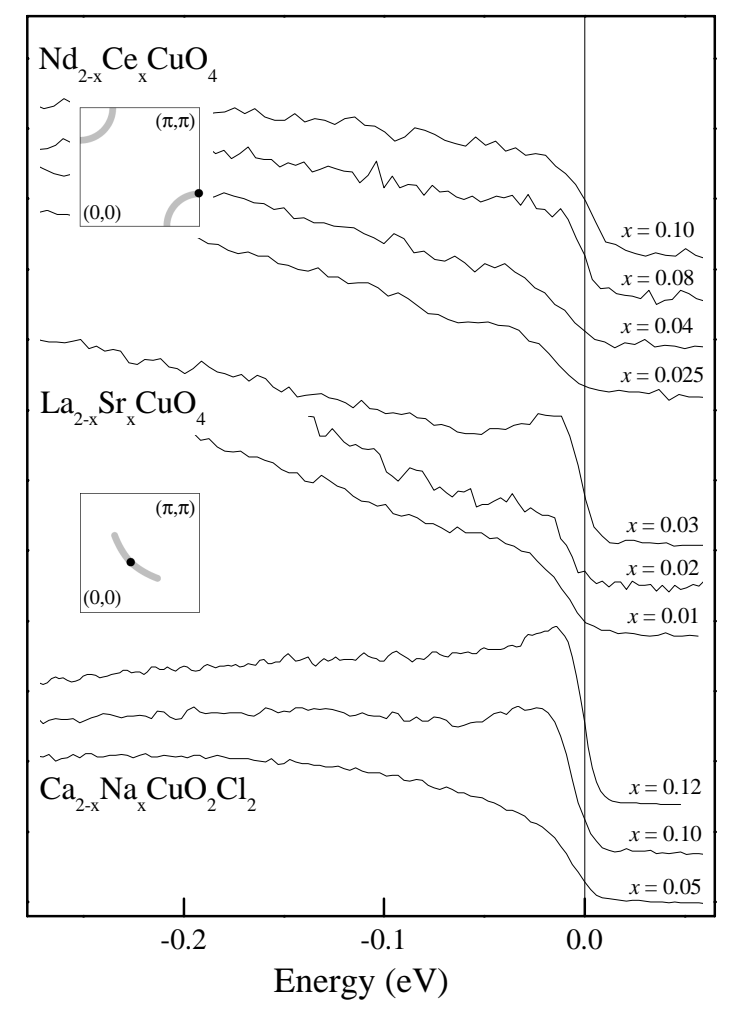

Figure A.2: LEG spectra from hole-doped $\mathrm{Ca}_{2-x} \mathrm{Na}_{x} \mathrm{CuO}_{2} \mathrm{Cl}_{2}$ and $\mathrm{La}_{2-x} \mathrm{Sr}_{x} \mathrm{CuO}_{4}$, and electron-doped $\mathrm{Nd}_{2-x} \mathrm{Ce}_{x} \mathrm{CuO}_{4}$. The bottom inset shows the wavevector of the spectra taken from $\mathrm{Ca}_{2-x} \mathrm{Na}_{x} \mathrm{CuO}_{2} \mathrm{Cl}_{2}$ and $\mathrm{La}_{2-x} \mathrm{Sr}_{x} \mathrm{CuO}_{4}$; the top shows $\mathrm{Nd}_{2-x} \mathrm{Ce}_{x} \mathrm{CuO}_{4}$. All data were taken at $15 \mathrm{~K}$.

similar to $\mathrm{Ca}_{2-x} \mathrm{Na}_{x} \mathrm{CuO}_{2} \mathrm{Cl}_{2}$, and the spectra are likewise taken from the $d_{x^{2}-y^{2}}$ nodal line. At a doping concentration of $x=0.01$, dispersive low-energy states are observed with a $\Delta_{\mathrm{LEG}}=9 \mathrm{meV}$. However, by $x=0.03$, this LEG has closed, to within our experimental resolution, and remains as such for higher concentrations, as studied in detail in [171] (which focuses on the metallic behavior for $x \geq 0.03$ ). In the case of lightly electron-doped $\mathrm{Nd}_{2-x} \mathrm{Ce}_{x} \mathrm{CuO}_{4}$, the first electron addition states appear as small electron pockets near $(\pi, 0)$ [26], in contrast to the hole-doped cuprates. In this case, we have observed the gap along these electron pockets with $\Delta_{\text {LEG }} \sim 16 \mathrm{meV}$ for $x=0.025$, while for $x=0.04, \Delta_{\mathrm{LEG}} \sim 8 \mathrm{meV}$, and closes to nearly zero by $x=0.08$; at higher concentrations $(x>0.10)$, the Fermi surface crosses over to a hole pocket centered at $(\pi, \pi)$. 
The measurement of a LEG is the canonical scheme by which excitation gaps have been typically determined by photoemission spectroscopy. It is difficult to ascertain the precise value of any excitation gap from the measurement of the gap without $a$ priori knowledge of the single-particle spectral function, $\mathcal{A}(k, \omega)$, making lineshape modelling potentially suspect. However, the LEG criterion has typically been successful in identifying the $d$-wave gap in the superconducting cuprates [46], charge density wave (CDW) gaps [215], and even small superconducting gaps in photoemission studies of conventional BCS materials such as $\mathrm{V}_{3} \mathrm{Si}$, $\mathrm{Nb}$, and $\mathrm{Pb}$ [237, 238]. Furthermore, our observation of finite LEGs in a wide variety of lineshapes and compounds suggests that this effect is not a misidentification due to a peculiar lineshape profile. Nevertheless, we should note that it is not inconceivable that in particular special instances, an ungapped spectral function may possibly give rise to a finite LEG in the ARPES lineshape (e.g. Luttinger liquids). All results were confirmed by multiple measurements on different sample batches. We have also utilized the method of symmetrization where $I_{\text {sym }}(\mathbf{k}, \omega)=I(\mathbf{k}, \omega)+I(\mathbf{k},-\omega)$, which has been demonstrated to be an effective procedure for determining the presence of Fermi crossings [239]. The results obtained from this method were qualitatively consistent with values obtained by taking the leading edge midpoints of the spectra.

Particular care was taken to avoid any electrostatic charging, a possible consideration due to the low-temperature insulating tendencies of these lightly doped samples. No change in $\Delta_{\text {LEG }}$ was observed when the photon flux was varied by a factor of 3 or greater. Also, the macroscopic sample surface quality for the lower doping concentrations was found to be comparable to more heavily doped samples, as determined from inspection by optical microscope and laser reflection. Finally, all three studied families are chemically pristine when undoped and must be alloyed towards higher doping levels. Therefore, the opening of this gap towards lower concentrations cannot be associated with a degradation in crystal quality, as would be the case for the bismuth based cuprates. We note that ARPES studies of irradiated $\mathrm{Bi}_{2} \mathrm{Sr}_{2} \mathrm{CaCu}_{2} \mathrm{O}_{8+\delta}$ have also shown a demonstrable effect of induced disorder on the low energy spectral lineshape [240].

The compositional and doping dependence of $\Delta_{\mathrm{LEG}}$ for all samples studied is 
summarized in Figure A.3 and is shown to be reproducible over numerous measurements. In all compounds, $\Delta_{\mathrm{LEG}}$ is largest at the lowest concentrations and closes with increasing doping. Despite the universal presence of this phenomenon, there exist obvious differences in the detailed behavior of each particular compound. In particular, the gap appears to close rapidly in $\mathrm{La}_{2-x} \mathrm{Sr}_{x} \mathrm{CuO}_{4}$. Another intriguing point is that for $\mathrm{Ca}_{1.9} \mathrm{Na}_{0.1} \mathrm{CuO}_{2} \mathrm{Cl}_{2}$, which has a $\mathrm{T}_{\mathrm{c}}$ onset of $13 \mathrm{~K}$, there still exists a small but finite $\Delta_{\mathrm{LEG}} \sim 3 \mathrm{meV}$ above $15 \mathrm{~K}$, the base temperature of our experiments. Interfamily variations in the behavior of $\Delta_{\text {LEG }}$ may not be unexpected, as many other physical properties exhibit considerable material-specific differences, including superconductivity and antiferromagnetism, and may depend on factors such as the chemical composition and crystal structure.

\section{A.3 Discussion and Implications of Experimental Results}

We now speculate on possible origins of the observed normal state gap. One very important consideration is that disorder is inherently manifest in the cuprates, as chemical substitution or intercalation is necessary for introducing carriers. At sufficiently low concentrations, the poor screening of these impurities should cause a strong disorder potential which may result in localization. The combination of disorder with long-range Coulomb interactions can produce a depression in the density of single-particle excitations at the chemical potential known as a Coulomb or "soft" gap [241], where the presence of repulsive electron-electron interactions necessitates a vanishing density of states at $\mathrm{E}_{\mathrm{F}}$ to ensure against an instability towards an excitonic ground state. The existence of disorder and localization may also be consistent with the reasonably broad peaks and edges, suggesting short lifetimes and/or breaking of translational symmetry resulting in poorly defined momentum eigenstates. Within this scenario, the reduction of $\Delta_{\mathrm{LEG}}$ with doping can be explained by the enhancement of screening. It should be emphasized that the presence of a Coulomb gap even in the presence of disorder is still a non-trivial result, since gapless insulating 


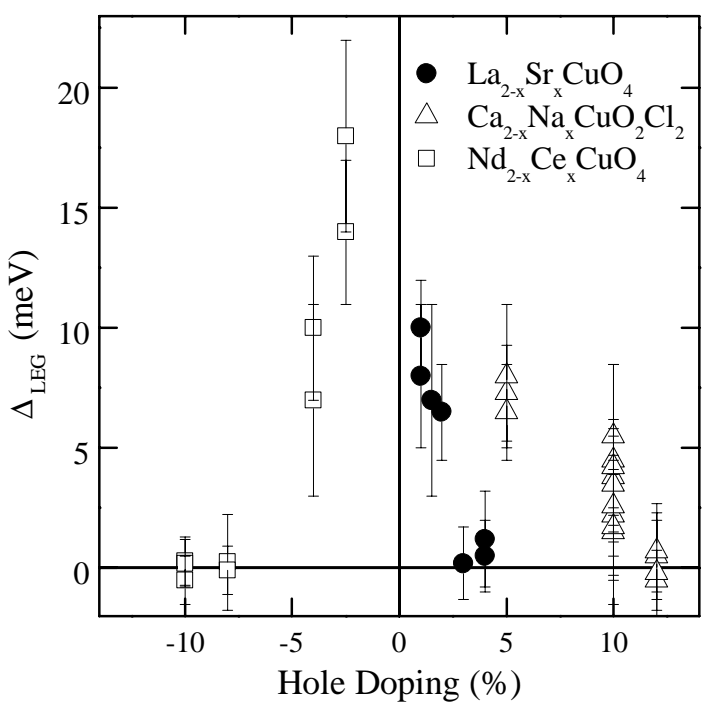

Figure A.3: Compilation of $\Delta_{\text {LEG }}$ measurements taken from all samples. Data from hole-doped samples were taken near $\left(\frac{\pi}{2}, \frac{\pi}{2}\right)$ while data from electron-doped samples were taken near $(\pi, 0)$, as shown in the insets in Figure A.2. All measurements were performed at $15 \mathrm{~K}$.

behavior can also occur (i.e. weak localization); a Coulomb gap in the lightly doped cuprates would be a clear indication of the strong electron-electron interactions in these systems. It is also possible that a Coulomb gap may exist in the lightly doped cuprates without the aid of chemical disorder.

Recent STM studies have found considerable electronic inhomogeneity in the cuprate superconductors [242, 205, 243]. In particular, results from Kohsaka et al. on $\mathrm{Ca}_{1.92} \mathrm{Na}_{0.08} \mathrm{CuO}_{2} \mathrm{Cl}_{2}$ have shown that this inhomogeneity persists to high energy scales [205], implying that the distribution of carriers varies strongly on nanometer length scales. A recent neutron scattering study of lightly doped $\mathrm{La}_{2-x} \mathrm{Sr}_{x} \mathrm{CuO}_{4}$ [244] also suggests the presence of electronic phase separation below $x=0.02$, close to where $\Delta_{\text {LEG }}$ vanishes. It is then possible that Coulomb blockade effects in mesoscopic systems such as granular metals [245] may be germane to this discussion. Photoemission results from ultrathin granular Pb films [246] and segmented one-dimensional systems [247], have been interpreted within such a framework. We note that the 
observed values of $\Delta_{\text {LEG }}$ appear rather small for Coulomb blockade in the nanometersized patches proposed for the cuprates, although additional effects such as the mutual screening of patches and photohole relaxation [248] may be mitigating factors. Moreover, it is unlikely that the charge modulations in the cuprates are so severe as to cause separation of the holes into truly insulating and metallic patches, and is more plausible that there are only small charge density modulations, which would then not result in such a large (or any) Coulomb gap. Whether this inhomogeneity is driven solely by the presence of chemical disorder or is an inherent property of the pristine $\mathrm{CuO}_{2}$ plane is still unclear. Nevertheless, both chemical disorder and/or the presence of intrinsic electronic inhomogeneity are plausible origins for a Coulomb gap which may account for our results. We also note that the presence of $\Delta_{\text {LEG }}$ naturally reconciles the existence of an insulating ground state, as determined from collective transport properties, with the development of finite spectral weight near $\mathrm{E}_{\mathrm{F}}$ as measured from single-particle spectroscopy. It is now clear that although the spectral intensity of excitations near $\mathrm{E}_{\mathrm{F}}$ grows as a function of doping, these low-energy states are additionally gapped, resulting in a charge and thermal insulator [234, 235].

Another intriguing possibility is that this may represent a signature of an alternate phase of matter. It has been proposed that the exotic normal state properties of the heavily underdoped cuprates may signify the presence of a competing order, such as a staggered flux phase [33] or charge/spin stripes [29]. It has also been established from neutron scattering that spin density wave (SDW) order exists in $\mathrm{La}_{1.6-x} \mathrm{Nd}_{0.4} \mathrm{Sr}_{x} \mathrm{CuO}_{4}$ [29] and $\mathrm{La}_{2-x} \mathrm{Sr}_{x} \mathrm{CuO}_{4+\delta}[249,250]$. The fact that we have consistently observed this effect in multiple families suggests that if $\Delta_{\text {LEG }}$ is due to a competing order, this order should be generic to the cuprate superconductors. In a competing order scenario, the doping dependence in Figure A.3 suggests that the strength of the competing phase decreases rapidly as a function of doping, similar to the behavior of the pseudogap. We note that the presence of chemical or electronic disorder does not necessarily preclude the existence of a competing order. Future experiments may help to clarify this situation. For instance, a systematic study of $\Delta_{\text {LEG }}$ with increasing chemical impurities, such as Zn or Ni substitution, or induced disorder [240], would elucidate the effects of disorder on this gap, and help to distinguish between a soft gap or a 
competing order scenario.

In summary, we have presented ARPES results revealing the existence of a finite gap over the entire Brillouin zone of the lightly doped cuprates in the lowtemperature normal state. This phenomenon was observed in both electron and hole-doped cuprates and was found to decrease as a function of carrier doping. We believe this effect is one of the keys underlying the novel superconductor-insulator transition in the lightly doped region of the phase diagram and may represent electronic inhomogeneity/disorder effects or a competing order parameter in the lightly doped regime. It is hoped that these results will spur future activity into developing a better understanding of the properties of the lightly doped cuprates. 


\section{Appendix B}

\section{Anomalous Photon Energy Dependence of ARPES Spectra from $\mathrm{Ca}_{2-x} \mathrm{Na}_{x} \mathrm{CuO}_{2} \mathrm{Cl}_{2}$}

\section{B.1 Background}

The very presence of the strong correlations which give rise to fascinating phenomena such as the Mott insulating state, the high transition temperature, pseudogap behavior, and electronic phase separation, may also provide significant complications for interpreting the ARPES spectra ${ }^{1}$. To date, the simplified approach outlined in Chapter 2 been employed for the analysis of ARPES data where it is assumed that the photoemission intensity from the $\mathrm{CuO}_{2}$ plane is directly proportional to the single particle spectral function, $\mathcal{A}(\mathbf{k}, \omega)$, and particularly that the coherent and coherent parts of the spectral function have the same photoelectron matrix elements. However, given their strong correlations and remarkable properties, the cuprates provide a unique opportunity to observe variations away from this conventional paradigm. Salient discrepancies may indicate the presence of previously overlooked phenomena. For instance, strong antiferromagnetic correlations are known to coexist with metallic

\footnotetext{
${ }^{1}$ This work forms the basis of a manuscript originally written in 2002, and will be modified and submitted to the Physical Review sometime in 2005.
} 
behavior and superconductivity [251,36], raising the question of whether the single particle spectrum is concurrently representative of both traits. It becomes imperative to develop a comprehensive understanding of precisely how such correlations can affect the photoemission spectra, especially as the technique progresses towards even higher levels of precision.

Here we report anomalous behavior of the ARPES spectra of the cuprate superconductor $\mathrm{Ca}_{2-x} \mathrm{Na}_{x} \mathrm{CuO}_{2} \mathrm{Cl}_{2}$ as a function of incident photon energy. In addition to strong modifications in the global momentum distribution of the intensity, $I(\mathbf{k}, \omega)$, we find inconsistent variations in extracted parameters such as the renormalized velocity and self-energy. More remarkably, we find that the photoemission lineshape itself exhibits prominent variations at low energies, contradictory to any simplified spectral function interpretations. Because these effects cannot be explained within the existing framework, we propose a new phenomenological dual-component model where the single particle spectrum is composed of distinct high and low energy excitations. The high energy excitations reflect the remnant incoherent spectral weight from the parent insulator (i.e. Zhang-Rice singlet), while the coherent low energy branch is derived from the more mobile doped hole states. These variations progress systematically with doping, further supporting our hypothesis. The physical basis for these effects is related to the sensitivity of the photoelectron matrix elements to disparities in the orbital configurations of the different initial state wavefunctions. Finally, we consider the potential influence of electronic inhomogeneity and how these findings may be generalized to other correlated systems.

\section{B.2 Experimental Details and Results}

Measurements were performed using synchrotron radiation with typical energy and angular resolutions of $13 \mathrm{meV}$ and $0.3^{\circ}$, respectively. The polarization of the photons had an in-plane component at $45^{\circ}$ to the $\mathrm{Cu}-\mathrm{O}$ bonds. Single crystals of $\mathrm{Ca}_{2-x} \mathrm{Na}_{x} \mathrm{CuO}_{2} \mathrm{Cl}_{2}(x=0,0.05,0.10,0.12)$ were grown using a high pressure flux method [114]. The $x=0.05$ composition was non-superconducting, while $x=0.10$ and 0.12 had $\mathrm{T}_{\mathrm{c}}$ 's of 13 and $22 \mathrm{~K}$, respectively $\left(\mathrm{T}_{\mathrm{c}, \mathrm{opt}}=28 \mathrm{~K}\right)$. Samples were cleaved 


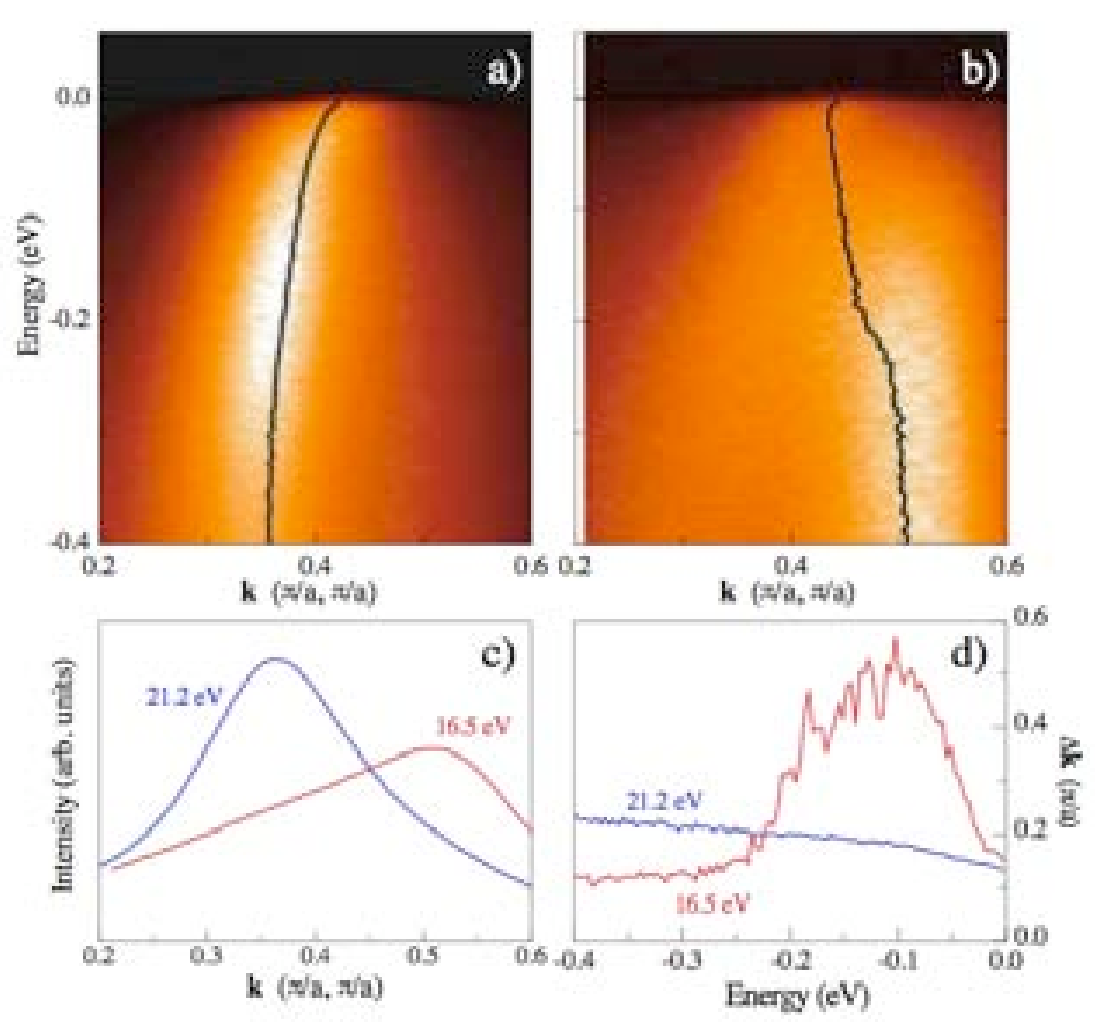

Figure B.1: Two-dimensional ARPES spectra are shown from $x=0.10$ along the $(0,0)-(\pi, \pi)$ direction at $h \nu=21.2$ (a) and $16.5 \mathrm{eV}(\mathrm{b})$. The MDC renormalized dispersion is overlaid in black. $\Delta k=\Sigma^{\prime \prime}(\mathbf{k}, \omega) / v_{\mathrm{F}}^{0}$ determined from (a) and (b) are shown in panel (c). The $\omega$-integrated intensity $(-500 \mathrm{meV}<\omega<0)$ as a function of wavevector is shown in panel (d), along with that from $x=0$ (dashed).

and measured at pressures better than $5 \times 10^{-11}$ torr at a base temperature of $15 \mathrm{~K}$. The results in this study did not exhibit any strong temperature dependence and were observed in both the normal and superconducting states. Results were reproducible over multiple samples from different growth batches.

In Figures B.1a and B.1b, we present raw data taken from underdoped $\mathrm{Ca}_{1.90} \mathrm{Na}_{0.10} \mathrm{CuO}_{2} \mathrm{Cl}_{2}$ along the $(0,0)-(\pi, \pi)$ symmetry line at $h \nu=21.2$ and $16.5 \mathrm{eV}$ on the same cleaved surface. Measurements were also performed with other photon energies between 16.5-32 eV, but for the purpose of clarity, only results from 21.2 and 16.5 $\mathrm{eV}$ will be discussed; other energies yielded results similar or intermediate to either 21.2 or $16.5 \mathrm{eV}$. The qualitative variations between Figures B.1a and B.1b are striking, 
as the $\mathbf{k}$-distribution of spectral weight is nearly reversed about $\mathbf{k}_{\mathrm{F}}=(0.43 \pi, 0.43 \pi)$. The momentum dependence of the integrated spectral weight $(-500 \mathrm{meV}<\omega<0)$ is shown in Figure B.1c and quantifies this global redistribution which was also observed for $x=0.05$ and 0.12 . In addition, while a well-defined low-energy branch is visible at $21.2 \mathrm{eV}$, this feature is obscured at $16.5 \mathrm{eV}$. Under the sudden approximation, the photoemission intensity can be expressed as $I(\mathbf{k}, \omega)=\mathrm{M}_{0}(\mathbf{k}, \mathbf{A}) f(\omega, \mathrm{T}) \mathcal{A}(\mathbf{k}, \omega)$ where $f(\omega, \mathrm{T})$ is the Fermi-Dirac distribution and $\mathrm{M}_{0}(\mathbf{k}, \mathbf{A})$ is the square of the oneelectron photoelectron matrix element, $\left|\left\langle\psi_{i}|\mathbf{A} \cdot \mathbf{p}| \psi_{f}\right\rangle\right|^{2}$, where $\psi_{i}$ and $\psi_{f}$ are the initial and final state wavefunctions. The direct proportionality between $I(\mathbf{k}, \omega)$ and $\mathcal{A}(\mathbf{k}, \omega)$, is what makes ARPES a powerful technique for studying many-body physics and in principle allows the extraction of physical quantities [82].

The apparent renormalized dispersion $\left(v=\frac{d \omega}{d k}\right)$ is determined from fitting the momentum distribution curves or MDCs $\left(I\left(\mathbf{k}, \omega_{0}\right)\right)$ using a standard procedure used in the cuprates outlined in [57] and [252] and overlaid as solid black lines in Figures 1a and 1b. The results obtained at the two photon energies differ dramatically; at $16.5 \mathrm{eV}$, the band appears to disperse inwards from $(\pi, \pi)$, contrary to data from 21.2 $\mathrm{eV}$ and theoretical and experimental expectations [40]. Additionally, the momentum uncertainty can be related to the imaginary part of the self-energy, $\Sigma^{\prime \prime}(\mathbf{k}, \omega)$, by $\Delta k=\Sigma^{\prime \prime}(\mathbf{k}, \omega) / v_{\mathrm{F}}^{0}$, where $v_{\mathrm{F}}^{0}$ is the unrenormalized band velocity, and $\Sigma^{\prime \prime}(\mathbf{k}, \omega)$ represents the inverse lifetime of quasiparticle excitations [82]. From the dramatic and unphysical changes in the extracted values of both $\Sigma^{\prime \prime}$ (Figure B.1d) and $v$, it becomes clear that some assumptions underpinning this basic analysis scheme possess serious inadequacies. For instance, the conversion of the raw intensity, $I(\mathbf{k}, \omega)$, to quantities such as $v$ and $\Sigma^{\prime \prime}$ relies on the assumption of a negligibly weak $\mathbf{k}$-dependence of $\mathrm{M}_{0}(\mathbf{k}, \mathbf{A})$. However, the dramatic variations in Figure B.1c clearly demonstrate the considerable momentum dependence of $\mathrm{M}_{0}$. An analogous redistribution of weight between $h \nu=21.2$ and $16.5 \mathrm{eV}$ was also observed in undoped $\mathrm{Ca}_{2} \mathrm{CuO}_{2} \mathrm{Cl}_{2}$ [253], and such similar behavior implies that the electronic character of the superconducting compound retains strong similarities to the undoped compound [253]. This suggests that the strong correlations inherent in the Mott insulator persist for $x>0$ and implies that our rudimentary analysis framework may be inadequate to accurately 
describe even the doped superconductors.

While the preceding discussion has focussed on the strong $\mathbf{k}$-dependence of $\mathrm{M}_{0}$, we now investigate the energy distribution curves or $\operatorname{EDCs}\left(I\left(\mathbf{k}_{0}, \omega\right)\right)$ and find unequivocal evidence for a multi-component electronic structure. Because EDCs are measured at single wavevector, their shapes are insensitive to any $\mathbf{k}$-dependence of $\mathrm{M}_{0}$, unlike the MDCs. The EDCs can then be expressed as $I\left(\mathbf{k}_{0}, \omega\right) \propto \mathcal{A}\left(\mathbf{k}_{0}, \omega\right)$ for the occupied states, after factoring in thermal and resolution broadening. The validity of this assumption is essential for theoretical attempts to model ARPES spectra and implies that $I\left(\mathbf{k}_{0}, \omega\right)$ should be invariant under changes in experimental conditions. To date, detailed ARPES studies regarding the photon energy $(h \nu)$ dependence of the doped cuprates have been performed only on bilayer $\mathrm{Bi}_{2} \mathrm{Sr}_{2} \mathrm{CaCu}_{2} \mathrm{O}_{8+\delta}$. Although sizable variations have been found in both the EDCs [254, 111] and Fermi surface [255, 256], these effects were explained by the presence of bonding and antibonding bilayer bands whose existence was already predicted by early band structure calculations for $\mathrm{Bi}_{2} \mathrm{Sr}_{2} \mathrm{CaCu}_{2} \mathrm{O}_{8+\delta}$ [257]. Nevertheless, all aforementioned studies implicitly assumed that the photoemission intensity from the single $\mathrm{CuO}_{2}$ sheet was comprised of only a solitary electronic component. $\mathrm{Ca}_{2-x} \mathrm{Na}_{x} \mathrm{CuO}_{2} \mathrm{Cl}_{2}$, with its single $\mathrm{CuO}_{2}$ layer per unit cell and simple tetragonal structure devoid of superlattice modulations, structural distortions, or surface reconstructions [114], provides us with an ideal model system with which to test this most fundamental assumption.

In Figure B.2, we present the $h \nu$ dependence of the EDC at $\mathbf{k}_{\mathrm{F}} \sim(0.43 \pi, 0.43 \pi)$ for $x=0.05,0.10$, and 0.12 . The discrepancy between $h \nu=21.2$ and $16.5 \mathrm{eV}$ is striking and provides direct evidence that the excitation spectrum of the $\mathrm{CuO}_{2}$ plane includes multiple components. From the raw data, it is clear that the low energy spectral weight $(\omega>-100 \mathrm{meV})$ is substantially enhanced at $h \nu=21.2 \mathrm{eV}$ over $16.5 \mathrm{eV}$ at all dopings. This substantial change is best exemplified at $x=0.10$ in Figure B.2b, where the small peak near $\mathrm{E}_{\mathrm{F}}$ is evident at $21.2 \mathrm{eV}$, but vanishes at $16.5 \mathrm{eV}$. The comparisons shown in each panel were performed on the same cleave and reproduced on other samples. It is evident from the raw data that the excitation spectrum from the $\mathrm{CuO}_{2}$ plane can no longer be considered as originating from a single initial state. However, in order to quantify these changes, we employ a simple phenomenological model. The 


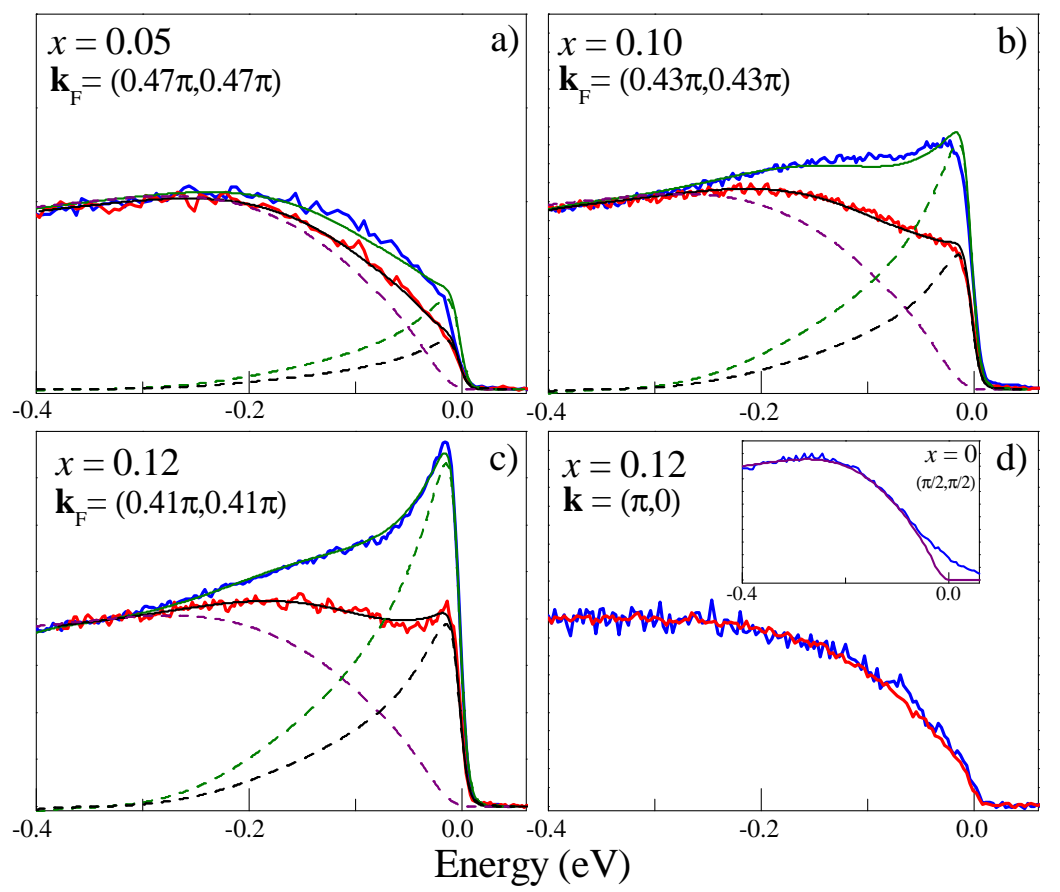

Figure B.2: EDC lineshapes for $x=0.05,0.10$, and 0.12 , with $h \nu=21.2$ (blue) and $16.5 \mathrm{eV}$ (red), are shown in (a-d). Fits for $21.2 \mathrm{eV}$ (solid green) and $16.5 \mathrm{eV}$ (solid black) are overlaid, along with the individual $\mathcal{L}_{\mathrm{p}}$ (dashed) and $\mathcal{L}_{\mathrm{h}}$ (dashed purple) components. In panel $(\mathrm{d})$, spectra at $(\pi, 0)$ from $x=0.12$ is shown. A comparison of $\mathcal{L}_{\mathrm{h}}$ with the shifted lineshape at $\left(\frac{\pi}{2}, \frac{\pi}{2}\right)$ from $x=0$ is shown in the inset.

lineshape can be approximately decomposed into two principal components: a broad hump at high energies, $\mathcal{L}_{\mathrm{h}}$, and a peak at low energies, $\mathcal{L}_{\mathrm{p}} \cdot \mathcal{L}_{\mathrm{h}}$ was derived from the lineshape of the Zhang-Rice singlet of the undoped insulator at $\mathbf{k}=\left(\frac{\pi}{2}, \frac{\pi}{2}\right)$ (Figure B.2d inset), while $\mathcal{L}_{\mathrm{p}}$ was a low-energy peak with a residual tail, both multiplied by $f(\omega, \mathrm{T})$. In the context of our work in Chapters 4 and $6, \mathcal{L}_{\mathrm{h}}$ would approximately correspond to the high-energy incoherent spectral weight (multi-boson sidebands), and $\mathcal{L}_{\mathrm{p}}$ is the low-energy coherent $\mathrm{QP}$ weight. To estimate the doping and photon energy dependence, we employ a basic two parameter fit where we use the same model lineshapes for $\mathcal{L}_{\mathrm{h}}$ and $\mathcal{L}_{\mathrm{p}}$ (dashed lines) and approximate the total lineshape as a linear superposition of the two components, $\mathcal{F}(\omega)=a \mathcal{L}_{\mathrm{h}}(\omega)+b \mathcal{L}_{\mathrm{p}}(\omega)$, where the scaling factors $a$ and $b$ are the only free parameters. The total fits are shown as black $(16.5 \mathrm{eV})$ and green $(21.2 \mathrm{eV})$ lines in Figure B.2 and agree well with the 
data. We should note that in this simplified analysis, we assume that $\mathcal{L}_{\mathrm{h}}$ and $\mathcal{L}_{\mathrm{p}}$ are doping independent. However, we know this is technically inaccurate from our work in Chapter 6. The strength of the electron-boson coupling constant should change strongly with doping, and $\mu$ also shifts, leading to strong changes in $\mathcal{L}_{\mathrm{h}}$ which have not been taken into account in this simplified model, therefore, these quantities should not be taken literally, but simply as an illustrative guide. Results from other photon energies $(18,19,25.5,28,32 \mathrm{eV})$ were not shown, but yielded comparable results. We note that some potentially relevant factors have been neglected in this simple model, such as the doping dependent changes in $\mathcal{L}_{\mathrm{h}}$ and $\mathcal{L}_{\mathrm{p}}$, or additional components. Nevertheless, we believe the reasonable agreement of this crude model with experiment further supports the general validity of our hypothesis.

The data in Figure B.2 suggests that both doping and photon energies play similar roles in determining the overall lineshape. This is evident from the similarity between the data from $x=0.10$ at $16.5 \mathrm{eV}$ with that from $x=0.05$ at $21.2 \mathrm{eV}$, as well as the resemblance between the spectra from $x=0.12$ at $16.5 \mathrm{eV}$ with that from $x=$ 0.10 at $21.2 \mathrm{eV}$. For that reason, we also assume a simple decomposition with $a=$ $\mathrm{M}_{\mathrm{h}} \cdot n_{\mathrm{h}}$ and $b=\mathrm{M}_{\mathrm{p}} \cdot n_{\mathrm{p}}$, where $\mathrm{M}_{\mathrm{i}}$ represents the doping independent, $h \nu$ dependent matrix element for component $\mathcal{L}_{\mathrm{i}}$, while $n_{\mathrm{i}}$ represents a doping dependent scaling factor. In Figure B.3a, we show the ratio, $r$ of $\frac{b}{a}=\frac{\mathrm{M}_{\mathrm{p}} n_{\mathrm{p}}}{\mathrm{M}_{\mathrm{h}} n_{\mathrm{h}}}$ (the weight of the peak over the hump) as a function of doping. It is striking that the doping dependence of $r(16.5)$ and $r(21.2)$ are nearly identical to within a factor. This is expected within our scenario where the doping dependence of $r$ should depend only on $n_{\mathrm{p}} / n_{\mathrm{h}}$. In the inset of Figure B.3a, we show $r(21.2) / r(16.5)$, which appears doping independent, consistent with our model.

\section{B.3 Discussion of Experimental Results}

These variations are direct evidence of a discrepancy in the microscopic character of the high and low energy $(\omega>-100 \mathrm{meV})$ excitations. At low doping, the high energy excitations comprise the large majority of the occupied spectral weight; in Fermi liquid terminology, the coherent fraction of $\mathcal{A}(\mathbf{k}, \omega)$ is small, or $Z \ll 1$. We 

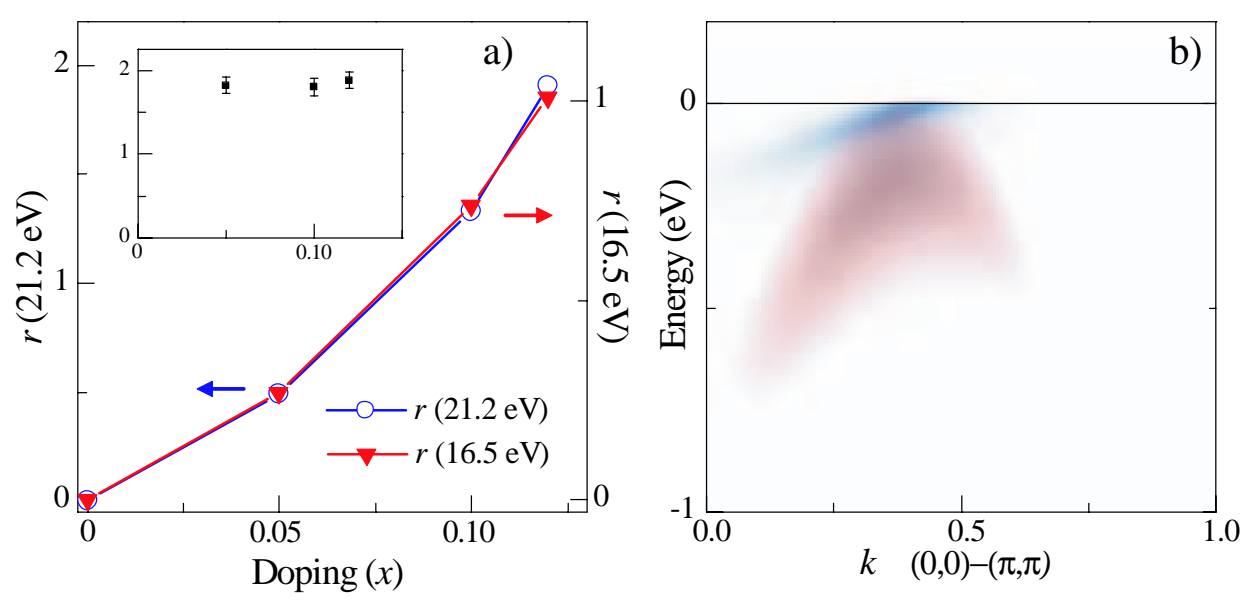

Figure B.3: Panel (a) shows $r=\frac{b}{a}$ as a function of $x$ for data at 21.2 and $16.5 \mathrm{eV}$. The ratio $\frac{r(21.2)}{r(16.5)}$ is shown in the inset. A momentum-dependent cartoon schematic of $\mathcal{L}_{\mathrm{h}}\left(\right.$ red) and $\mathcal{L}_{\mathrm{p}}$ (blue) is shown in (b).

also find a doping induced transfer of spectral weight from high to low energies, resulting in an increase in the peak with $x$, as shown in Figure B.3a. These results may also be consistent with the coexistence of Drude weight $\left(\mathcal{L}_{\mathrm{p}}\right)$ and residual chargetransfer excitations (originating from $\mathcal{L}_{\mathrm{h}}$ ) in optical conductivity measurements of the underdoped cuprates [37]. A cartoon schematic of this two-component model is shown in Figure B.3b, where the excitation branches for $\mathcal{L}_{\mathrm{h}}$ and $\mathcal{L}_{\mathrm{p}}$ are shown in red and blue, respectively. The shape and dispersion of $\mathcal{L}_{\mathrm{h}}$ was found to closely match the Zhang-Rice singlet in the undoped insulator [258], while the sharp peak $\mathcal{L}_{\mathrm{p}}$ is reminiscent of a more quasiparticle-like state. Our model is also consistent with the lack of $h \nu$ dependence at $(\pi, 0)$ in Figure B.2d, where there are no well-defined low energy excitations. In this case, the $(\pi, 0)$ lineshape is better described with a single component demonstrating that the low energy spectral weight displays strong anisotropy, seen in studies of the "nodal metal" in $\mathrm{La}_{2-x} \mathrm{Sr}_{x} \mathrm{CuO}_{4}$ [171].

The physical origin of the discrepancies in $\mathrm{M}_{h}$ and $\mathrm{M}_{p}$ is likely the different wavefunctions of the states comprising $\mathcal{L}_{\mathrm{p}}$ and $\mathcal{L}_{\mathrm{h}}$. While early work by Zhang and Rice showed that the $\mathrm{Cu} 3 d_{x^{2}-y^{2}}$ and $\mathrm{O} 2 p_{x, y}$ states could be effectively reduced to a single band [24], this band still possesses strong hybridization between the $\mathrm{Cu} 3 d_{x^{2}-y^{2}}$ and $\mathrm{O} 2 p_{x, y}$ states. Because the initial state wavefunction, $\psi_{i}$ is composed of a linear 
combination of orbitals, $\left|\left\langle\psi_{i}|\mathbf{A} \cdot \mathbf{p}| \psi_{f}\right\rangle\right|^{2}$ will depend crucially on their hybridized configuration. Even subtle changes in the coefficients and relative phases of the orbitals becomes extremely important due to the interference of cross-terms the computation of the squared matrix element. Therefore, the difference between $\mathcal{L}_{\mathrm{h}}$ being a more correlated, Zhang-Rice singlet-like state and $\mathcal{L}_{\mathrm{p}}$ being a more QP-like state can likely result in the observed discrepancy in $\mathrm{M}_{h}$ and $\mathrm{M}_{p}$. Simple changes in the atomic crosssections alone cannot explain these effects, as the total $\mathrm{Cu} 3 d$ and $\mathrm{O} 2 p$ cross-sections change only negligibly within the relevant photon energy range [259]. Moreover, the suppression of $\mathcal{L}_{\mathrm{p}}$ at lower photon energies is unlikely to be due to a breakdown of the sudden approximation; upon approaching the adiabatic limit, the spectral weight accumulates into a single peak, opposite to what is observed

One natural explanation for our observations may be the presence of electronic phase separation where effectively undoped regions give rise to the broad hump reminiscent of the parent insulator, while hole-rich regions produce the dispersive nodal peak responsible for transport properties. Moreover, the doping dependent transfer of spectral weight to $\mathcal{L}_{\mathrm{p}}$ is consistent with this model, as one expects the fractional area occupied by hole-rich states to increase with doping. Although this may offer one straightforward premise, other possibilities should not be excluded.

In the context of our work in Chapter 6, one natural possibility is that the wavefunction heavily dressed, "polaronic" quasiparticle becomes heavily mixed with the bosonic field dressing the photohole. The quasiparticle is still fermionic in nature, but it may begin to take on certain aspects of the bosonic field, for instance, in terms of its symmetry, and this may be then be evidenced through the photoelectron matrix elements. It is possible that this is somewhat analogous to the observation of "shape resonances" in molecular photoemission, where the photoelectron intensity profile has a strong dependence on the incoming photon energy, and this is a possibility that needs more investigation given the potential for very strong electron-boson coupling in many strongly correlated systems.

Another possibility is based not on electron-boson coupling but on calculations from dynamical mean-field theory which predict that upon doping a Mott insulator, the spectral function can possess both delocalized quasiparticle excitations as well as 
broader localized (i.e. Hubbard-like) features [189]. The transition from insulator to metal is then signified by a transfer of spectral weight between the two, and has been borne out in the present study. We believe that these results can be generalized to other systems. Since the $\mathrm{CuO}_{2}$ planes are generic to the high-temperature superconductors, similar effects should be expected in the other cuprates, although they might be difficult to distinguish from other complications (bilayer splitting, superstructure, orthrhombicity) in more structurally complex compounds. In fact, we believe these findings to be generic to many strongly correlated materials where Mott-Hubbard physics and phase separation are believed to be important, such as the nickelates and manganites, as well as systems undergoing metal-insulator transitions.

In conclusion, we have demonstrated for the first time that complex effects exist in the photoemission intensity arising from the single $\mathrm{CuO}_{2}$ plane. We propose that the effects of correlation result in the presence of two disparate excitation branches, a lower Hubbard band-like feature at high energies and a more well-defined delocalized state at low energy. Given the amount of effort currently employed in modelling ARPES spectra and extracting quantitative parameters, discovering fundamental deviations and failings of the existing framework are imperative for all future studies. We postulate that similar complications may be generic to ARPES studies of other strongly correlated systems, and systems with strong electron-boson coupling (and therefore small $Z$ ). It is hoped that the findings from this study provide a more mature understanding of the complex nature of the photoemission process in the cuprates and other correlated systems. 


\section{Appendix $\mathrm{C}$}

\section{Surface Electronic Structure of $\mathrm{Sr}_{2} \mathrm{RuO}_{4}$}

\section{C.1 Background}

Following the discovery of superconductivity $(\mathrm{SC})$ at $1 \mathrm{~K}$ in the layered perovskite $\mathrm{Sr}_{2} \mathrm{RuO}_{4}$, [260] the exact nature of its $\mathrm{SC}$ pairing mechanism has attracted a great deal of interest. ${ }^{1}$ While it shares the same structure as the archetypal cuprate parent compound $\mathrm{La}_{2} \mathrm{CuO}_{4}, \mathrm{RuO}_{2}$ planes replace the $\mathrm{CuO}_{2}$ planes thus resulting in an anisotropic Fermi liquid [261] instead of a strongly correlated charge transfer insulator. Furthermore, there is evidence that $\mathrm{Sr}_{2} \mathrm{RuO}_{4}$ exhibits spin-triplet pairing with a $p$-wave order parameter,[262] as opposed to the spin-singlet, $d$-wave symmetry found in the cuprates. Although it is now widely believed that the unconventional nature of $\mathrm{SC}$ in this compound is mediated by spin fluctuations, the exact nature of this interaction is still unresolved. Originally, it was suggested that ferromagnetic (FM) spin fluctuations were responsible for mediating the SC as inferred from theoretical calculations, [263] NMR measurements,[264] and ferromagnetism in closely related $\mathrm{SrRuO}_{3}$. However, more recent evidence has suggested that this simple picture may

\footnotetext{
${ }^{1}$ The vast majority of this appendix has been published in K.M. Shen et al., Physical Review B 64, 180502(R) (2001).
} 
be incomplete. Antiferromagnetism (AFM) in $\mathrm{Ca}_{2} \mathrm{RuO}_{4}$, the observation of incommensurate peaks at $\mathbf{Q}=(0.6 \pi, 0.6 \pi, 0)$ by neutron scattering, [265] and calculations which show strong nesting at $\mathbf{Q}=(2 \pi / 3,2 \pi / 3,0)$ [263] all seem to imply AFM correlations should not be neglected, leaving the nature of SC open to speculation.

Recently, an analysis of low-energy electron diffraction data from $\mathrm{Sr}_{2} \mathrm{RuO}_{4}$ indicated that a $\sqrt{2} \times \sqrt{2}$ reconstruction was induced by the freezing of a soft zone boundary phonon into a static lattice distortion at the surface, and comparisons with band structure calculations predicted that the resulting surface was FM. [120] This possibility was also speculated upon in a recent ARPES study by our group, [121] as well as in earlier theoretical calculations.[266] If FM does exist on the surface of $\mathrm{Sr}_{2} \mathrm{RuO}_{4}$, such a result should be indicative of strong ferromagnetic tendencies in the bulk and thus possibly relevant to microscopic theories which describe the mechanism of SC. This speculation becomes even more intriguing in light of recent STM measurements[267] which suggest the opening of a superconducting gap with $\mathrm{T}_{c}=$ $1.4 \mathrm{~K}$, perhaps hinting that the surface layer may be superconducting, and raises the possibility that the surface of $\mathrm{Sr}_{2} \mathrm{RuO}_{4}$ may exhibit the rare coexistence of $\mathrm{SC}$ and FM. However, as this proposed surface FM has never been confirmed, it becomes imperative to reinvestigate the surface electronic structure to definitively verify or exclude surface FM.

Here, we present a detailed, high-resolution ARPES study of the surface electronic structure of $\mathrm{Sr}_{2} \mathrm{RuO}_{4}$. While our earlier work [121] ascertained that the bulk Fermi surface (FS) topology extracted by ARPES was indeed in excellent agreement with both theory [268, 269] and de Haas-van Alphen (dHvA) results, [261] the precise nature of the surface-derived states, which could be nonmagnetic (NM) or FM, remained ambiguous. In particular, our earlier depiction of the surface electronic structure failed to explicate the presence of the intense, surface-derived peak at $(\pi, 0)$, leaving us to speculate that its existence could be a possible manifestation of surface FM. To clarify this uncertainty, we have performed a comprehensive ARPES study with various photon energies and polarizations in conjunction with detailed band structure calculations which now account for the surface reconstruction. By comparing these calculations with our ARPES data, we conclude that our results are consistent with 

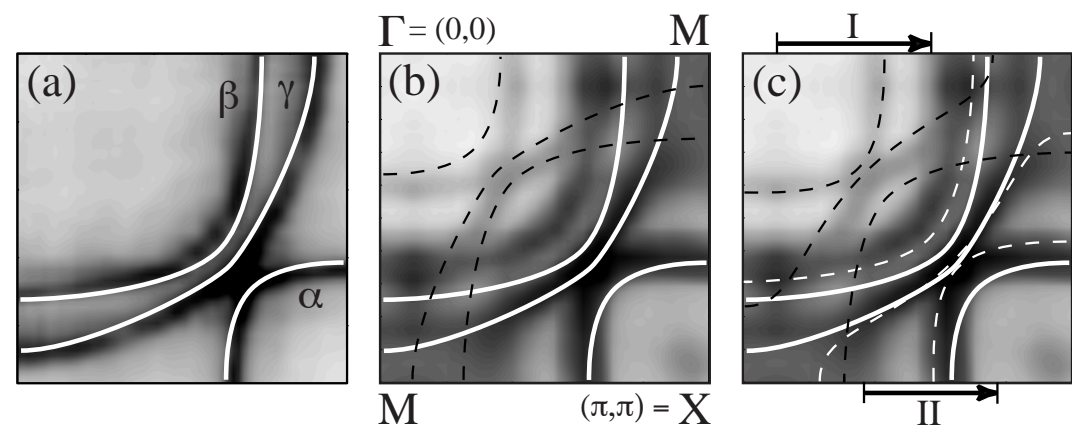

Figure C.1: (a) $\mathrm{E}_{F}$ intensity map of $\mathrm{Sr}_{2} \mathrm{RuO}_{4}$ cleaved at $180 \mathrm{~K}$ and measured at 10 $\mathrm{K}$ with $h \nu=28 \mathrm{eV}$ and overlaid theoretical FSes. (b) and (c) show an intensity map from a sample cleaved and measured at $10 \mathrm{~K}$. (b) shows calculated bulk FSes (white) with trivial folded FSes (dashed), while (c) shows calculated $6^{\circ} \mathrm{NM}$ reconstruction with both primary and folded surface FSes (dashed white and dashed black).

the NM scenario and exhibit no experimentally detectable trace of surface FM down to $10 \mathrm{~K}$.

\section{C.2 Experimental Details and Results}

Data was taken at the synchrotron with typical resolutions of $\Delta \mathrm{E}<13 \mathrm{meV}$ and $\Delta \theta \approx 0.2^{\circ} . \mathrm{Sr}_{2} \mathrm{RuO}_{4}$ single crystals were first aligned by Laue diffraction and then cleaved in situ at a pressure of better than $5 \times 10^{-11}$ torr and at various temperatures described below. All spectra were measured at $10 \mathrm{~K}$ in both the first and second Brillouin zones; surface features showed slight enhancement in the second zone.

Figure C.1a shows an $\mathrm{E}_{F}$ intensity map (integrated signal within $\mathrm{E}_{F} \pm 5 \mathrm{meV}$ ) of a sample cleaved at $180 \mathrm{~K}$ and measured at $10 \mathrm{~K}$. As discussed in [121], cleaving the sample at elevated temperatures preferentially suppresses the surface intensity; we speculate that the increased rate of thermally activated oxygen diffusion results in a more disordered surface layer. The resulting intensity map thereby primarily reflects the bulk contribution, and the calculated bulk FSes from [269] are overlaid and in excellent agreement. When cleaving at lower temperatures, the surface states were well preserved and also apparent in our data, in addition to the bulk states. This additional surface contribution is clearly visible in the intensity maps in Figures 
C.1b and C.1c and somewhat complicates the situation. Our original conjecture, in [121] and shown in Figure C.1b, accounted for the surface states by considering them to be the same as those of the bulk, except for a rigid folding due to the $\sqrt{2} \times \sqrt{2}$ surface reconstruction; the reconstruction arises from rotations of the $\mathrm{RuO}_{6}$ surface octahedra which cause a doubling of the surface unit cell.[120] Despite the approximate agreement, this overly simplistic picture fails to explain the origin of the strong peak (bottom of Figure C.2b) at M, which influenced earlier ARPES reports to erroneously designate the bulk $\gamma$-FS as hole-like.[270, 271] This apparent discrepancy also led us to initially posit that surface FM might be responsible for this state at M. However, after calculating the precise effects of the surface distortion on the band structure, we find that the NM reconstruction alone can potentially drive the surface $\gamma$-FS hole-like, thus explaining the peak at M; this more accurate NM scenario is depicted in Figure C.1c.

Nonetheless, since surface FM might still account for some of the experimentally observed features, it becomes crucial to examine the surface states in greater detail. In particular, surface FM would cause the surface states to split into minority and majority bands, effectively doubling the number of surface-derived bands. In order to distinguish between the NM and FM scenarios, we focus on ARPES spectra taken along lines I and II in Figure C.1c. For the NM surface, we would expect to see one band, $\alpha_{F}$, crossing along I and two crossings, $\alpha_{S}$ and $\alpha_{B}$, along II. Any additional bands beyond those predicted for the NM surface would be strong evidence for surface FM, and should be readily apparent in the ARPES data.

To address this issue, we first focus on spectra taken along I, shown in Figure C.2a, using $24 \mathrm{eV}$ photons polarized along the $\mathrm{Ru}-\mathrm{O}$ bond direction; different photon energies and polarizations yielded similar results. This region is particularly suitable for an investigation into potential surface FM since it is far removed from the bulk electronic states. Examining the energy distribution curves (EDCs) in Figure C.2a, we see only a single electronic state from the folded $\alpha$-FS, denoted as $\alpha_{F}$, as is expected from the NM scenario shown in Figure C.1c. Conversely, additional bands reflecting the exchange splitting would be expected for a FM surface. In Figure C.2d, we show a momentum distribution curve (MDC) of data from C.2a, where the photoemitted 

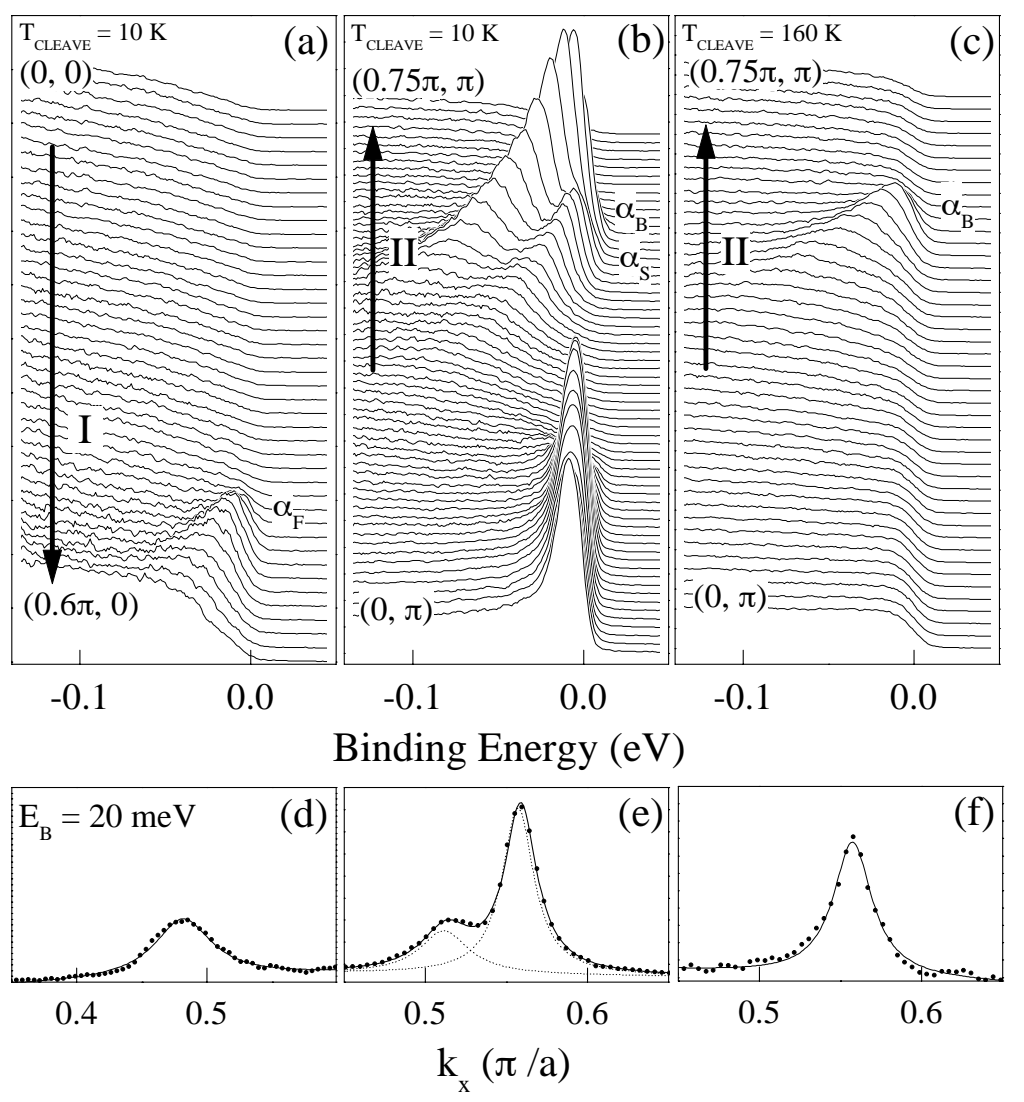

Figure C.2: EDCs in panels (a), (b), and (c) with along cuts I and II shown in Figure C.1. Corresponding MDCs at $\mathrm{E}_{B}=20 \mathrm{meV}$ taken from (a), (b), and (c) are shown in panels (d), (e) and (f), respectively. Data from (a), (b), (d), and (e) were taken on a sample cleaved at $10 \mathrm{~K}$, while data from (c) and (f) were taken on a sample cleaved at $160 \mathrm{~K} . \alpha_{F}, \alpha_{S}$, and $\alpha_{B}$ refer to folded, surface, and bulk $\alpha$ bands, respectively.

electron intensity is displayed as a function of momentum at a fixed binding energy of $\mathrm{E}_{B}=20 \mathrm{meV}$ and fitted to a single Lorentzian lineshape on a linear background. By analyzing our data in this fashion, we are able to track the dispersion of $\alpha_{F}$ yielding $v_{F}^{F}=0.7 \mathrm{eV} \cdot \AA$. Therefore our measurements along I yield only a single surface band, consistent with the nonmagnetic scenario of Figure C.1c.

To further reinforce this result, we now consider data along II shown in Figure C.2b, taken under the same conditions as I, but in the second zone. In both the EDCs and MDCs, one can clearly observe two distinct peaks. By fitting the MDCs to a double-Lorentzian form, shown in Figure C.2e, and tracking their dispersion to 


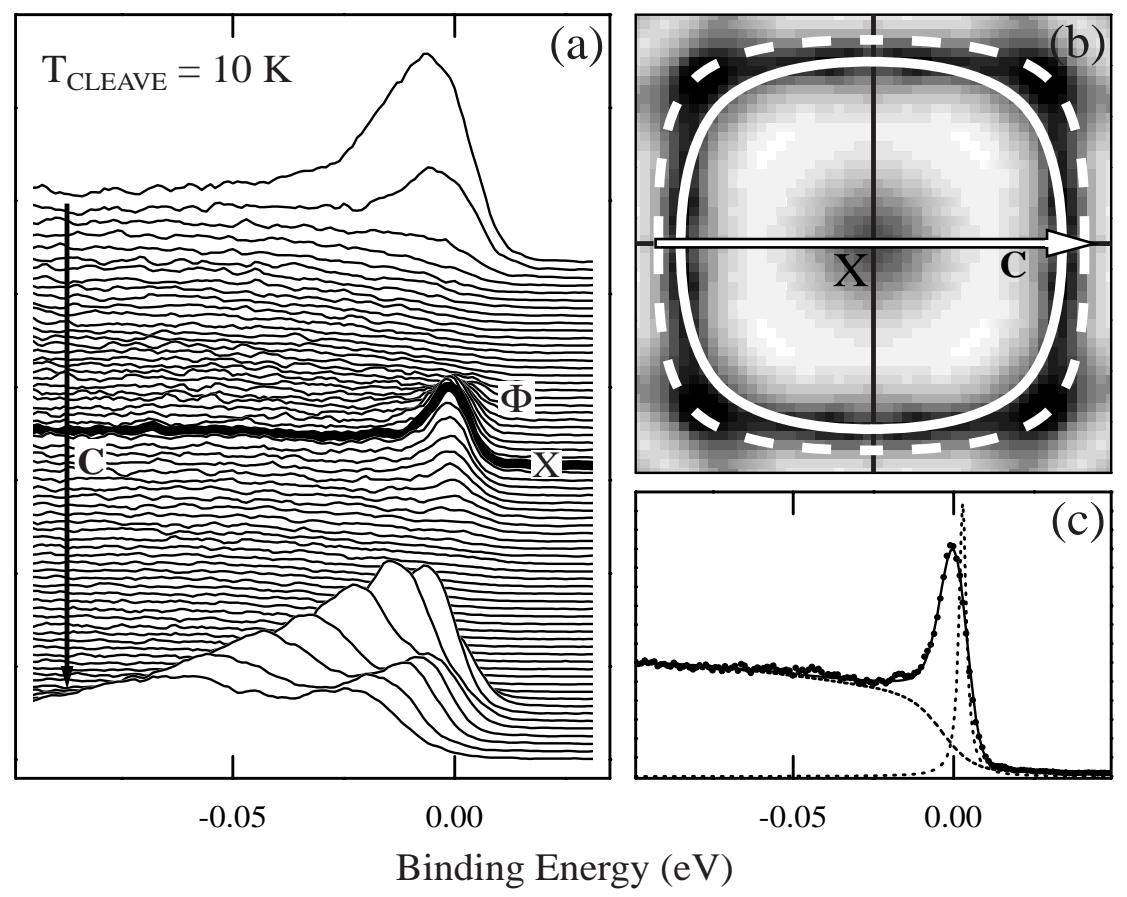

Figure C.3: ARPES data taken near $\mathrm{X}$ with $h \nu=24 \mathrm{eV}$ at $10 \mathrm{~K}$ on a sample cleaved $10 \mathrm{~K}$. EDCs in (a) were taken along cut C. (b) shows an $\mathrm{E}_{F}$ intensity map $( \pm 5 \mathrm{meV})$ around the region of the bulk and surface $\alpha$-FSes, shown in solid and dashed white, respectively. (c) shows the EDC from X and the corresponding fit. The background curve and the Lorentzian used in fitting are also shown as dotted lines.

$\mathrm{E}_{F}$, one can determine the Fermi velocities of the two bands. From this analysis, we determine the velocity of the first band, $\alpha_{B}$, to be $v_{F}^{B}=1.1 \mathrm{eV} \cdot \AA$, while for the second, $\alpha_{S}, v_{F}^{S}=0.7 \mathrm{eV} \cdot \AA$. On another sample cleaved at $160 \mathrm{~K}$ with the measurement taken in otherwise identical conditions, $\alpha_{S}$ is suppressed, as shown in Figure C.2c, and the remaining state is the bulk-derived $\alpha_{B}$; both $\alpha_{B}$ features in C.2b and C.2c have the same $v_{F}$ and Fermi crossing position. Also note that cleaving at elevated temperatures completely suppresses the strong peak at the bottom of C.2b, which is also responsible for the weight at $\mathrm{M}$ in Figures C.1b and C.1c. Furthermore, we are able to conclude that $\alpha_{F}$ is simply the folded counterpart of the surface-derived $\alpha_{S}$, and not the counterpart of the bulk-derived $\alpha_{B}$, since $\alpha_{S}$ and $\alpha_{F}$ have matching $v_{F}$ and Fermi crossings in the reduced zone. Therefore, the absence of additional bands along II is consistent with our results from I and our conclusion of a NM surface. 
Examining EDCs taken over the entire zone, virtually all observed surface states can be well accounted for by considering only a NM surface. The only unexpected feature was a small peak localized around $\mathrm{X}$, hereafter denoted as $\Phi$, as shown in Figures C.3a and C.3b, which was most strongly enhanced at $h \nu=24 \mathrm{eV}$. Close inspection of the spectrum at $\mathrm{X}$ in Figure C.3c reveals that the peak position is located at $\mathrm{E}_{F}( \pm 1 \mathrm{meV})$ and the leading edge is $6 \mathrm{meV}$ above $\mathrm{E}_{F}$, indicating that this peak originates from above $\mathrm{E}_{F}$; the peak in the EDCs is the product of the rising tail of the quasiparticle peak and the falling edge of the Fermi-Dirac function. This was confirmed by fitting the data using a background taken from $(0.8 \pi, \pi)$ and a Lorentzian peak, both multiplied by a Fermi-Dirac function and convolved with our resolution $(\Delta \mathrm{E}=8 \mathrm{meV})$, allowing us to estimate a peak position of $3 \mathrm{meV}$ above $\mathrm{E}_{F}$ and an intrinsic FWHM of $3 \mathrm{meV}$. Although there may be some slight inaccuracies in the fitting procedure, all attempts to fit the spectra to a below- $\mathrm{E}_{F}$ peak proved wholly unsuccessful. Moreover, since no corresponding band can be seen to disperse from below $\mathrm{E}_{F}$, we can conclude that $\Phi$ arises from an unoccupied band whose minimum at $\mathrm{X}$ almost grazes $\mathrm{E}_{F}$. Naively, one might infer that this somewhat unexpected feature could be interpreted as evidence for surface FM. However, as will be discussed below, our calculations, in fact, even predict the appearance of $\Phi$, which arises from the distortion of the surface layer.

\section{C.3 Discussion and Comparison with Theory}

In order to gain deeper insight into the effects of the surface reconstruction, we have performed both NM and FM calculations similar to those reported in [269], but including rotations by a fixed angle in all $\mathrm{RuO}_{2}$ planes, resulting in a monoclinic $C 2 / m$ symmetry. We will hereafter refer to these rotated bulk calculations as pertaining to the surface, and this assumption can be justified because of the extremely 2D nature of the electronic structure; any effects from the surface termination should be far weaker than those of the rotations of the octahedra, and is demonstrated by the excellent agreement of our NM rotated calculations with the corresponding surface calculations performed by Fang. [120, 272] 
Figure C.4a shows the results of NM calculations along the tetragonal M-X direction for rotations of $\theta=0^{\circ}$ (bulk) and $\theta=6^{\circ}$ (NM surface). An angle of $6^{\circ}$ was chosen since it is within the error bars of the structural data [120] and also gives good agreement with our ARPES data, especially the placement of $\Phi$. The NM surface and bulk bands are shown in Figure C.4a, and the experimentally determined values are overlaid and in good agreement with theory. We also note that our estimate of the quasiparticle renormalization $v_{F}^{\text {calc }} / v_{F}^{B}=3.2$ for the bulk $\alpha$-FS along M-X is in excellent agreement with $\mathrm{m}^{*} / m_{\text {band }}=3.3$ from dHvA [261], and also close to theoretical estimates of $\approx 2.5$. [273] Calculations for $\theta=6^{\circ}$ produce the dashed FSes in Figure C.1c and the rotation induces various effects.

First, the extended van Hove singularity (evHs) at $\mathrm{M}$, which is $60 \mathrm{meV}$ above $\mathrm{E}_{F}$ in the bulk calculations, is pushed $40 \mathrm{meV}$ below $\mathrm{E}_{F}$ due to the repulsion between the $d_{x y}$ and $d_{3 z^{2}-r^{2}}$ bands, which is allowed only in the lowered symmetry of the distorted surface. This results in the topology of the surface $\gamma$-FS changed from electron-like to hole-like, as shown in Figure C.1c, also concurring with independent calculations from [274]. Furthermore, the dispersion of this feature, in agreement with [270] and [271], is consistent with the saddlepoint topology predicted by theory.

Secondly, the lower symmetry on the surface also allows for hybridization between the $d_{x y}$ and $d_{x^{2}-y^{2}}$ bands forbidden in the tetragonal symmetry. In the distorted structure, these two states are both at the now-equivalent $\Gamma / \mathrm{X}$ point of the downfolded zone and repel each other. Furthermore, rotations disrupt the $\mathrm{Ru}-\mathrm{O} p d \sigma$ hopping more strongly than the $p d \pi$ hopping and thus the $d_{x^{2}-y^{2}}$ band moves down relative to the $d_{x y}$ band. Both effects together lead to the formation of a strongly mixed state at the $\Gamma / \mathrm{X}$ point which moves down rapidly and gains more $d_{x^{2}-y^{2}}$ character with rotation angle. While it is $300 \mathrm{meV}$ above $\mathrm{E}_{F}$ for $\theta=0^{\circ}$, it crosses $\mathrm{E}_{F}$ for $\theta$ $=7^{\circ}$, and is the origin of $\Phi$. Although $\Phi$ was not observed at $\Gamma$, this absence is not surprising when considering the unfavorable photoemission matrix elements due to the significant $d_{x^{2}-y^{2}}$ and $d_{x y}$ character of this state.

The effects of FM on the surface electronic structure were evaluated by performing constrained fixed spin moment calculations for the $6^{\circ}$ surface with an imposed magnetization of $1 \mu_{B} / \mathrm{Ru}$, a value consistent with [120]. The corresponding FM surface 

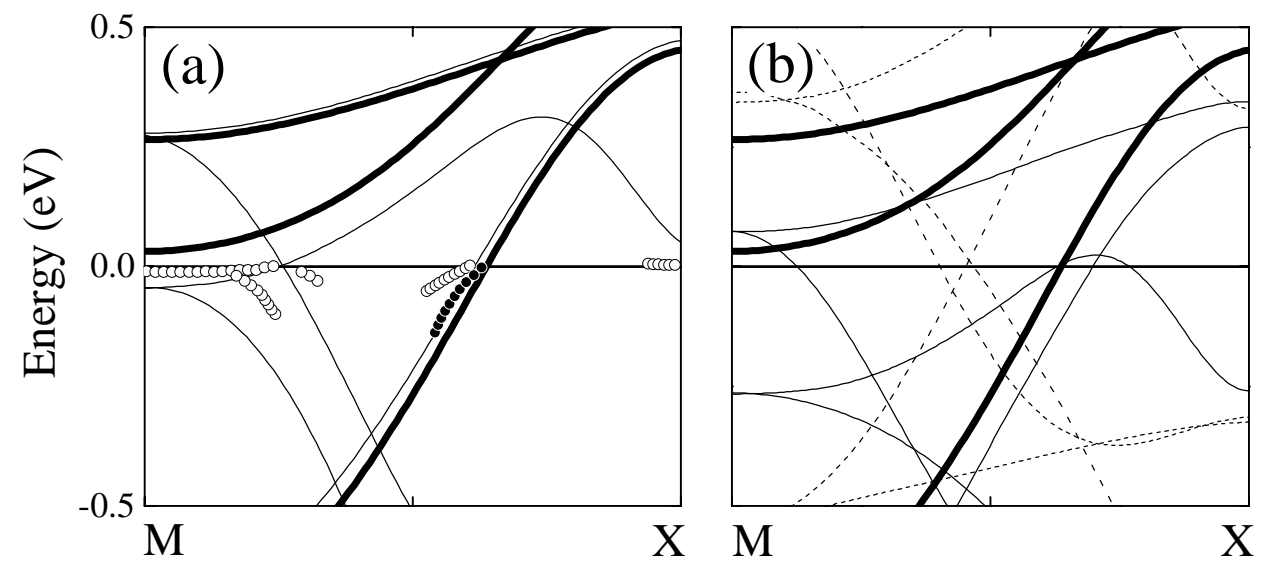

Figure C.4: (a) : Band structure calculations along M-X for bulk and $6^{\circ} \mathrm{NM}$ surface (thick and thin lines) along with ARPES dispersion for bulk and surface states (solid and open circles). (b) : Bulk, minority surface, and majority surface bands in thick, thin, and dashed lines for a $6^{\circ} \mathrm{FM}$ surface.

calculation is shown in Figure C.4b and is radically different from what is measured experimentally; for instance, both the evHs at M and the bottom of the $d_{x y} / d_{x^{2}-y^{2}}$ band at X are absent. Regardless of the particular details of the calculations, such as the position of the chemical potential and the bands, even the number of bands expected and measured is in disagreement, thus favoring the NM scenario, in contrast to the earlier speculation by our group [121] and Matzdorf et al. [120]

Although exact comparisons between the theoretical calculations and the ARPES data can be somewhat difficult due to the significant electron-electron interactions, the qualitative comparison of the ARPES data with the general behavior of the calculated electronic structure should be robust. The earlier conclusion of surface FM [120] was based on the comparison of structural data $\left(\theta=9^{\circ} \pm 3^{\circ}\right)$ to magnetic band structure calculations. However, the error bars in the structural data are comparable to the spread in the calculated rotation angles for a NM (6.5 ), AFM (6.5 ), and FM surface $\left(9^{\circ}\right)$, leaving room open for alternative interpretations of the data. Furthermore, the generalized gradient approximation employed in the aforementioned calculations may be inclined to overestimate the tendency towards magnetism, and even incorrectly predicts ferromagnetism in bulk $\mathrm{Sr}_{2} \mathrm{RuO}_{4}$. [266] We can place a maximum upper 
bound on the strength of any existing FM by considering our experimental resolution and the width of the quasiparticle peaks. If we assume that both $\alpha_{S}$ and $\alpha_{F}$ were comprised of a pair of extremely weakly split FM bands, we are able to put an upper bound of $\mathrm{E}_{\text {exch }} \approx 15 \mathrm{meV}$, which is much smaller than the predicted FM exchange splitting of $\approx 500 \mathrm{meV}$. [266, 272] Using this value of $\mathrm{E}_{\text {exch }} \approx 15 \mathrm{meV}$ results in an upper bound for the spin polarization of $<0.05 \mu_{B} / \mathrm{Ru}$, much weaker than predicted theoretically.

In conclusion, we have isolated and directly studied the surface-derived electronic states in $\mathrm{Sr}_{2} \mathrm{RuO}_{4}$ by ARPES. By comparison with detailed band structure calculations, we find that the origin of the ARPES features can be simply explained by considering the effect of a nonmagnetic surface reconstruction on the electronic structure, with no evidence of surface FM. 


\section{Appendix D}

\section{Electronic Structure of the high-temperature BCS superconductor $\mathrm{MgB}_{2}$}

\section{D.1 Background}

The discovery of superconductivity in $\mathrm{MgB}_{2}$, with the surprisingly high critical temperature $\left(T_{c}\right)$ of $39 \mathrm{~K}[7]$, has garnered a tremendous amount of interest $[275]^{1}$. Concerning the nature of the superconducting pairing mechanism, there has been considerable speculation, particularly regarding whether superconductivity could be explained within the Bardeen-Cooper-Schrieffer framework [6]. On the one hand, on the basis of experiments performed on isotope substituted material it has been shown that the electron-phonon interaction is indeed important to the pairing in $\mathrm{MgB}_{2}$ $[276,277]$; on the other hand, $T_{c}$ is considerably higher than what many would have originally believed possible as a result of conventional electron-phonon interaction alone $[278,279]$. This has prompted the question of whether additional factors, such as electron-electron correlations [280, 281, 282], may conspire to raise the superconducting transition temperature. In order to quantitatively address this important

\footnotetext{
${ }^{1}$ The vast majority of this appendix has been published in H. Uchiyama, K.M. Shen, et al., Physical Review Letters 88, 157002 (2002).
} 
issue, an experimental determination of the electronic structure of $\mathrm{MgB}_{2}$ is crucial. In fact, the strength of the electronic correlations can be estimated from the renormalization of the overall electronic bandwidth, Fermi velocity, and effective mass with respect to the values predicted by band theory (in the extreme case, electronic correlations could result in the opening of a so-called Mott-Hubbard gap in systems that on the basis of the bare electron counting are expected to be metallic [283]; this corresponds to the complete breakdown of the independent particle picture which, however, is clearly not realized in $\mathrm{MgB}_{2}$ ).

Here we report the first ARPES measurements performed on the first synthesized single crystals of $\mathrm{MgB}_{2}$ [284]. We find a good overall agreement between the experimental data and the results of existing band structure calculations [285, 286, 287, 288, 289], which gives a good basis for understanding the fundamental properties of this material. Furthermore, the good agreement indicates that electron-electron correlation effects are very weak. In turn, this implies that the electronic structure of $\mathrm{MgB}_{2}$ is of a conventional nature and electron-electron interactions are of little importance to superconductivity.

\section{D.2 Experimental Details and Results}

Single crystals of $\mathrm{MgB}_{2}$ were grown in the quasi-ternary $\mathrm{Mg}-\mathrm{MgB}_{2}-\mathrm{BN}$ system under 5-6 GPa at $1600{ }^{\circ} \mathrm{C}$. Several single crystals with typical dimensions of $0.3 \times 0.3 \times 0.1$ $\mathrm{mm}^{3}$ were selected for this study. The single-crystallinity was confirmed by four circle x-ray diffraction. Both resistivity and magnetization measurements verified that the crystals exhibit superconductivity at $38 \mathrm{~K}$ with a narrow transition width of $0.3 \mathrm{~K}$ [284]. The ARPES measurements were performed at the synchrotron with a total energy resolution of better than $40 \mathrm{meV}$ and an angular resolution of $0.3^{\circ}$. In this study, higher energy resolution was sacrificed in order to obtain reasonable counting statistics on the small single crystals. The samples were first aligned by Laue diffraction and then cleaved in situ along the $a-b$ plane at a pressure better than $5 \times 10^{-11}$ torr and a temperature of about $10 \mathrm{~K}$. Due to the observed rapid degradation of the sample surface, all data were taken within 5 hours of cleaving. 
Reproducible results were obtained on different cleaves.

The ARPES spectra were collected parallel to the high symmetry directions $\Gamma M$ and $\Gamma \mathrm{K}$ [where $\Gamma=(0,0,0), \mathrm{M}=(\pi, 0,0)$, and $\mathrm{K}=(2 / 3 \pi, 2 / 3 \pi, 0)]$, using a photon energy of $h \nu=28 \mathrm{eV}$ and incident electric-field polarization perpendicular to each respective symmetry direction (see Figure D.1a). Along $\Gamma M$, the polar emission angle was changed from $0^{\circ}$ (normal emission) to $28^{\circ}$ in the plane defined by the surface normal and the [100] axis; along $\Gamma \mathrm{K}$ it was changed from $0^{\circ}$ to $33^{\circ}$ in the plane defined by the surface normal and the [110] axis. The normal to the sample surface was determined by a standard laser-reflection procedure, and the fine alignment of the sample with respect to the electron analyzer was verified by checking the symmetry of the detected electronic bands with respect to the normal-emission direction. Since $\mathrm{MgB}_{2}$ is characterized by a three dimensional electronic structure and the electronic bands are predicted to show strong dispersion along the $z$ axis $[285,286]$, we performed photon-energy dependence measurements at normal emission in order to estimate the corresponding $k_{z}$ value for each given incident photon energy [72]. The photoemission cross section was found to be maximum at $h \nu=28 \mathrm{eV}$ in the explored range of 17-28 $\mathrm{eV}$, and decreased rapidly and monotonically upon progressively lowering the photon energy. However, due to the combination of large background and low intensity it was not possible to observe a clear electronic dispersion as a function of incident photon energy at normal emission. Therefore, we could not determine experimentally the exact $k_{z}$-coordinates for the ARPES data presented here.

The spectra in Figures D.1b and D.1c are representative energy distribution curves (EDCs) taken along the $\Gamma(\mathrm{A})-\mathrm{M}(\mathrm{L})$ and $\Gamma(\mathrm{A})-\mathrm{K}(\mathrm{H})$ directions, respectively. Several dispersive bands can be observed, as emphasized by the colored dots. The positions of these markers were determined from the combined analysis of EDCs and second derivative plots, which will be described in more details below. Although the data have been taken well below $T_{c}$, in which case a few meV superconducting gap should be open along the normal state Fermi surface, due to the insufficient energy resolution used in the present experiment the gap has not been resolved and all the detected bands appear to reach and cross the Fermi energy. Near the $\mathrm{K}(\mathrm{H})$ and $\mathrm{M}(\mathrm{L})$ points, we observed a strong dispersive peak that approaches $E_{F}$, as emphasized by the blue 


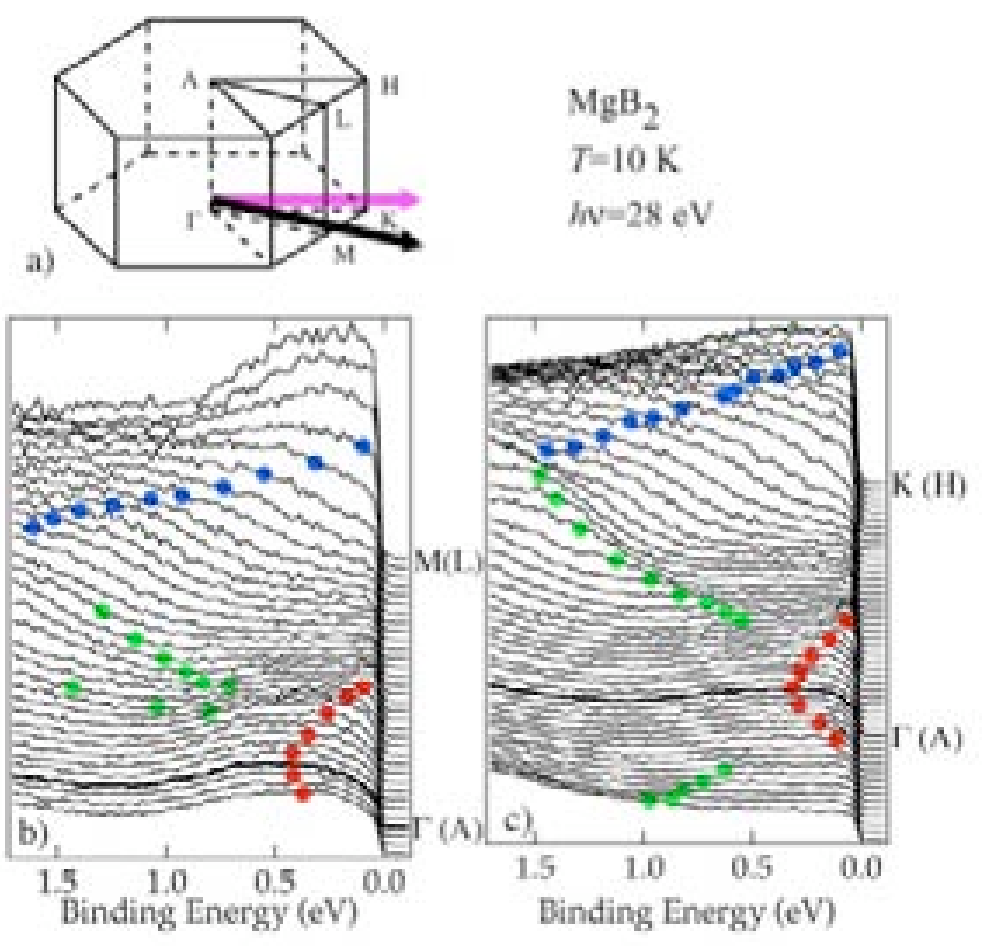

Figure D.1: (b,c) $\mathrm{MgB}_{2}$ ARPES spectra from along the $\Gamma(\mathrm{A})-\mathrm{M}(\mathrm{L})$ and $\Gamma(\mathrm{A})-\mathrm{K}(\mathrm{H})$ directions, as indicated by the arrows in the Brillouin zone sketch (a). The B $2 p_{z}$ and $2 p_{x, y}$ bands are marked in blue and green, respectively, while the red dots denote the surface state centered around $\Gamma(\mathrm{A})$.

dots. Along $\Gamma(\mathrm{A})-\mathrm{K}(\mathrm{H})$ another feature, marked in green, is approaching $E_{F}$ near the $\Gamma(\mathrm{A})$ point. Along the $\Gamma(\mathrm{A})-\mathrm{M}(\mathrm{L})$ direction the corresponding feature is very weak, although subtle changes in the lineshape can be discerned and are marked in green. Finally, along both the $\Gamma(\mathrm{A})-\mathrm{K}(\mathrm{H})$ and $\Gamma(\mathrm{A})-\mathrm{M}(\mathrm{L})$ directions, a small parabolic-like band is centered at the $\Gamma(\mathrm{A})$ point and reaches $E_{F}$ near $\left(\pi / 4,0, k_{z}\right)$ and $\left(\pi / 6, \pi / 6, k_{z}\right)$, respectively.

To more effectively visualize the ARPES data in the context of band dispersions, the image plots of the second derivative of the EDCs are shown in Figure D.2a. By taking the second derivative with respect to the binding energy of the raw ARPES data shown in Figures D.1b and D.1c, the relative contrast of the detected features can be enhanced, especially for those bands which are characterized by a very broad 
structure in the EDCs. The spurious intensity in the second derivative plots, which does not have a correspondent in the raw data and therefore does not represent any true feature, is likely due to higher sensitivity to statistical noise. The bands identified in Figures D.1b and D.1c are evident in the image plots of Figure D.2a, where the overlaid colored solid lines emphasize the experimentally determined electronic bands. In particular, the two weak features in the raw data along the $\Gamma(\mathrm{A})-\mathrm{M}(\mathrm{L})$ direction are much more pronounced in the second derivative image plots. Note that the EDCs and second derivative image plots emphasize different aspects of the data; in Figure D.1 what catches one's eye is the intensity of the EDCs, while in Figure D.2a is the change in slope of the EDCs, independent of the relative intensity. Together with the enhancement of the photoemission intensity at the M point most probably due to matrix element effects, this is the reason for the apparent disagreement in Figure D.1b between the dispersion of the B $2 p_{z}$ band as inferred from the EDCs and from their second derivative (blue dots).

A comparison of the features observed along $\Gamma \mathrm{M}$ and $\Gamma \mathrm{K}$ with the results of band structure calculations $[285,286]$ for $k_{z} \approx 0$ is presented in Figure D.2b, which shows a remarkable agreement between experiment and theory in the whole studied energy range (i.e., up to $2.5 \mathrm{eV}$ binding energy). To account for the $k_{z}$ uncertainty, our results are compared to calculations projected in $k_{z}$ between $k_{z}=0$ and $k_{z}= \pm 0.14 \pi$, which are represented by broad lines in Figure D.2b (it is worth emphasizing that, within the emission-angle range used in the present experiment, the percentage change in $k_{z}$ is considerably smaller than in $k_{\|}$). Since the agreement is completely lost for other values of $k_{z}$, we conclude that at normal emission with $28 \mathrm{eV}$ photons we are close to the plane containing the $\Gamma$ point, as far as the $z$-dispersion is concerned. Thus, our angular cuts lay close to the $\Gamma \mathrm{M}$ and $\Gamma \mathrm{K}$ lines. From Figure D.2b, we can clearly assign the feature marked in blue to the B $2 p_{z}(\pi)$ band, and those marked in green to the $\mathrm{B} 2 p_{x, y}(\sigma)$ bands. Of the two $\sigma$-bands predicted along $\Gamma \mathrm{K}$, only one is experimentally observed. However, as in the calculations the two bands lie close in energy, the broad feature we observe may likely result from the superposition of the two. In addition, although the $2 p_{x, y}$ bands along $\Gamma \mathrm{M}$ are weak in both the EDCs and the second derivative image plots, the close agreement of these features with the 


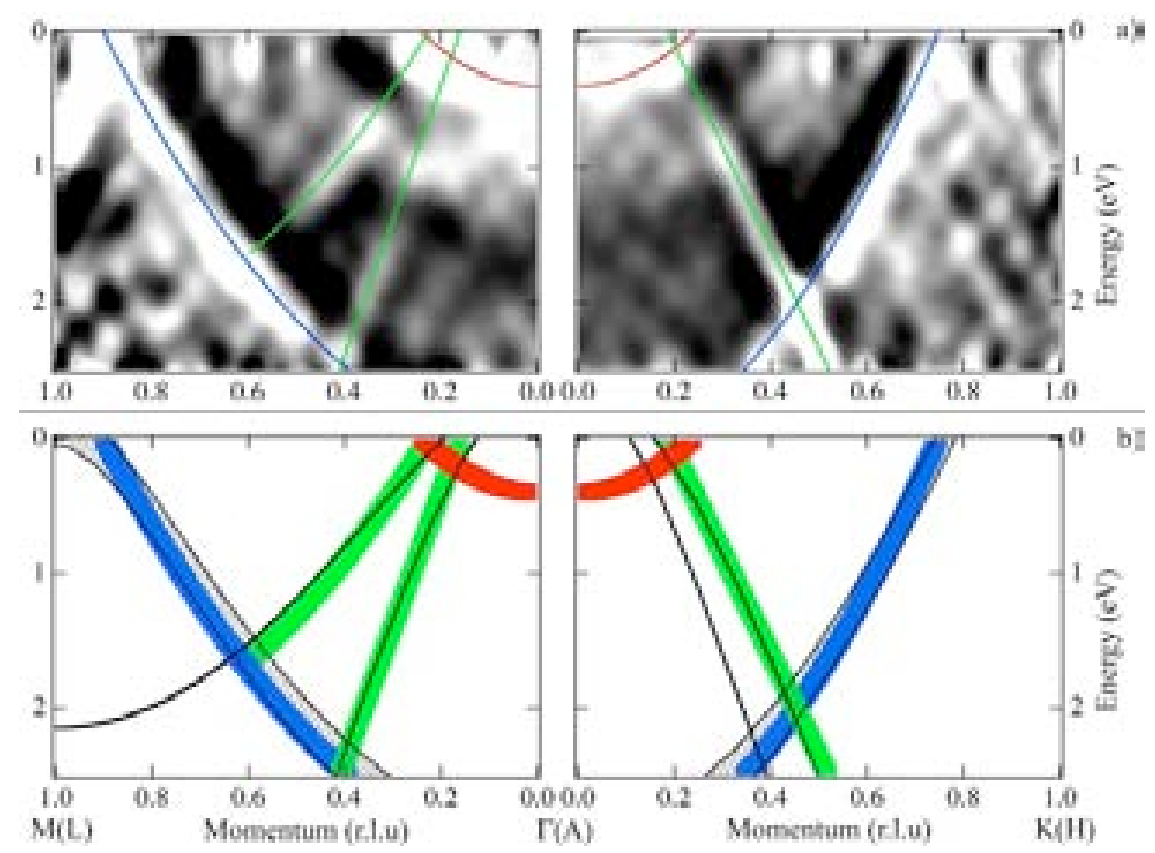

Figure D.2: (a) Second-derivative plots of the energy distribution curves (EDCs) shown in Figure D.1. The data were smoothed in both energy and momentum before taking the second derivative with respect to the binding energy. Colored lines emphasize the detected electronic bands, consistently with Figure D.1. (b) Comparison between theoretical (black) and experimental (color) results. The width of the theoretical lines represents the projection of $k_{z}$ values from 0 to $\pm 0.14 \pi$.

theoretical calculations lends strong support to our identification. We note that a similar contrast in the photoemission intensity for $\sigma$ and $\pi$ bands is observed also on graphite [290], which possesses an electronic structure somewhat similar to the one of $\mathrm{MgB}_{2}$, and appears to be a consequence of matrix element effects.

Finally, we turn our attention to the electronic state centered around the $\Gamma$ point and marked in red in Figures D.1, D.2, and D.3. From the comparison with the results of band structure calculations, one can conclude that there is no theoretically predicted bulk band which would correspond to this particular feature. It is entirely possible that this feature originates from a surface electronic state. The existence of surface states, which is a consequence of the breaking of translational symmetry at the crystal surface, is a rather universal phenomenon and occurs in many simple materials such as $\mathrm{Au}, \mathrm{Ag}, \mathrm{Cu}, \mathrm{Si}$, and graphite [72]. Therefore, the existence of such 


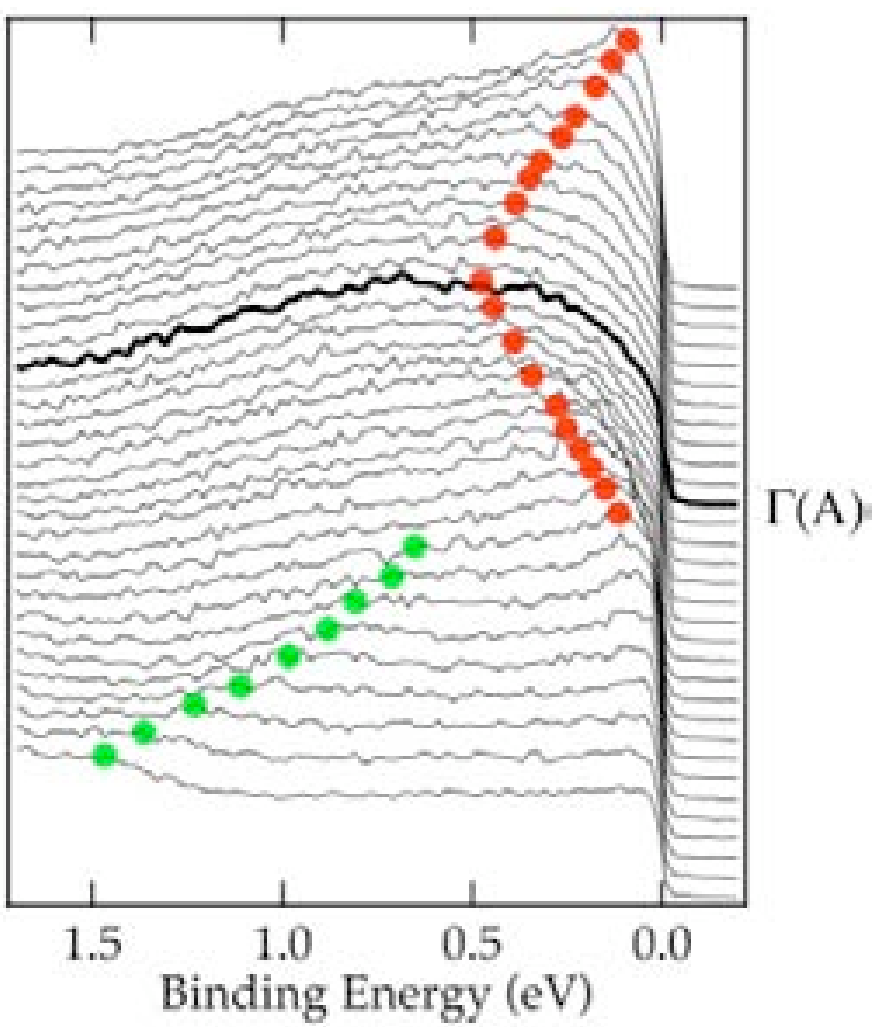

Figure D.3: Enlarged view of the EDCs taken close to the $\Gamma$ point, along the $\Gamma \mathrm{K}$ direction (see also Figure D.1b), which shows the surface state (red) and the B $2 p_{x, y}$ band (green).

a surface state in $\mathrm{MgB}_{2}$ is not surprising, especially around the $\Gamma$ point where there is a gap in the $k_{z}$-projected bulk band structure [285, 286]. The close agreement between all the other detected features and the results of band structure calculations also lends credence to our assignment of this feature to a surface state, as opposed to an unexpected bulk band. In addition, calculations for the electronic structure of the $\mathrm{MgB}_{2}(0001)$ surface predicted the existence of several surface and image potential states for both B- and Mg-terminated surfaces [287, 288, 289]. In particular, there is an overall good agreement between ARPES and theoretical results for the Mg-terminated surface, as indicated by self-consistent ab initio calculations of the electronic structure $[287,288]$, as well as one-step model calculations of the ARPES 
intensity in which the position of the surface potential is treated as a phenomenological parameter [289]. At this point, it should be noted that many measurements of the superconducting gap magnitude in $\mathrm{MgB}_{2}$ were performed by surface-sensitive techniques such as tunnelling [291, 292, 293, 294] and angle-integrated photoemission spectroscopy [295, 296]; the presence of a surface state could potentially affect the interpretations of these results, especially as far as the issue of a multiple gap in the bulk electronic structure is concerned.

\section{D.3 Discussion}

The close overall agreement of our experimentally determined band dispersions with the theoretical calculations seems to indicate that the effects of electron-electron correlations in this material are weak. In particular, electronic correlations would typically result in an overall renormalization or narrowing of the total electronic bandwidth as compared to the calculated value, and in an effective mass larger than the expected band mass. Clearly, as indicated by our investigation, this behavior is not observed in the ARPES data up to binding energies as high as $2.5 \mathrm{eV}$, thus suggesting that electronic correlations in $\mathrm{MgB}_{2}$ are fairly unimportant and that this material can be well-described by conventional band theory. At low binding energies, it is in principle possible to directly estimate from the ARPES spectra the interaction, if any, of the quasiparticles with collective modes such as, for example, lattice vibrations in electron-phonon coupled systems [297]. This interaction might result in a change of the electronic velocity along the quasiparticle dispersion and/or in an additional pole structure in the EDCs at the characteristic phonon energies [297]. These effects might actually be expected in the present case, given that $\mathrm{MgB}_{2}$ is a relatively strongcoupling superconductor in which the phonon density of states extends up to $100 \mathrm{meV}$ [298]. However, no clear behavior of this kind was observed in our measurements. On the other hand, no definitive conclusion can be drawn on this issue on the basis of the present data because the signatures of an interaction between quasiparticles and collective modes could be easily masked by the broadness of the experimental lineshapes. This, in turn, might be a consequence of the finite $k_{z}$ dispersion and/or of a 
considerable disorder of the cleaved surface at the atomic scale. Further scrutiny of this issue is therefore required.

In conclusion, we have studied the electronic structure of $\mathrm{MgB}_{2}$ by ARPES focusing, in particular, on the electronic dispersion near the Fermi level. Two $\sigma$-bands and one $\pi$-band were observed, as expected for the bulk electronic structure. The presence of multiple bands must be the origin of the complicated physical properties of $\mathrm{MgB}_{2}$, such as the extremely small but remarkably temperature dependent Hall coefficient [275]. An additional band was observed around the $\Gamma$ point and assigned to a surface state of the Mg-terminated cleaved surface. The close agreement between experimental and theoretical results for all detected features supports the view that $\mathrm{MgB}_{2}$ is a conventional metal in which electronic correlations are weak and superconductivity is likely of conventional origin. 


\section{Bibliography}

[1] R.B. Laughlin and D. Pines. Proc. Natl. Acad. Sci., 97:28, 2000.

[2] H.K. Onnes. Leiden Comm., 120b, 122c, 124c, 1911.

[3] W. Meissner and R. Ochsenfeld. Naturwissenschaften, 21:787, 1933.

[4] F. London and H. London. Proc. Roy. Soc., A149:71, 1935.

[5] V.L. Ginzburg and L.D. Landau. Zh. Eksperim. i Teor. Fiz., 20:1064, 1950.

[6] J. Bardeen, L.N. Cooper, and J.R. Schrieffer. Phys. Rev., 108:1175, 1957.

[7] J. Nagamatsu et al. Nature, 410:63, 2001.

[8] J.G. Bednorz and K.A. Mueller. Z. Phys. B, 64:189, 1986.

[9] M.K. Wu et al. Phys. Rev. Lett., 58:908, 1987.

[10] Y. Tokura, H. Takagi, and S. Uchida. Nature, 337:345, 1989.

[11] F. Bloch. Z. Phys, 52:555, 1928.

[12] L.D. Landau. Sov. Phys. JETP, 3:920, 1956.

[13] L.D. Landau. Sov. Phys. JETP, 5:101, 1957.

[14] L.D. Landau. Sov. Phys. JETP, 8:70, 1958.

[15] A.J. Schofield. Contemp. Phys., 40:95, 1999.

[16] J.M. Luttinger et al. Physical Review, 119:1153, 1960. 
[17] J.H. deBoer and E.J.E. Verwey. Proc. Phys. Soc. London, Sect. A, 49:59, 1937.

[18] N.F. Mott. Proc. Phys. Soc. London, Ser. A, 49:72, 1937.

[19] E. Wigner. Physical Review, 46:1002, 1934.

[20] N.F. Mott. Proc. Phys. Soc. London, Ser. A, 62:416, 1949.

[21] N.F. Mott. Can. J. Phys., 34:1356, 1956.

[22] N.F. Mott. Philos. Mag., 6:287, 1961.

[23] J. Zaanen, G. A. Sawatzky, and J. W. Allen. Phys. Rev. Lett., 55:418, 1985.

[24] F.C. Zhang and T.M. Rice. Phys. Rev. B, 37:3759, 1988.

[25] N.B. Brookes et al. Phys. Rev. Lett., 87:237003, 2001.

[26] N.P. Armitage et al. Phys. Rev. Lett., 88:257001, 2002.

[27] N.P. Armitage. Doping the Copper-Oxygen Planes with Electrons : The View with Photoemission. PhD thesis, Stanford University, 2001.

[28] M. Kastner et al. Rev. Mod. Phys., 70:897, 1998.

[29] J. M. Tranquada et al. Nature, 375:561, 1995.

[30] J. Zaanen and O. Gunnarsson. Phys. Rev. B, 40:7391, 1989.

[31] V.J. Emery, S.A. Kivelson, and H.Q. Lin. Phys. Rev. Lett., 64:475, 1990.

[32] S.A. Kivelson et al. Rev. Mod. Phys., 75:1201, 2003.

[33] I. Affleck and J.B. Marston. Phys. Rev. B, 37:3774, 1988.

[34] T. Timusk and B. Statt. Rep. Prog. Phys., 62:61, 1999.

[35] G.S. Boebinger et al. Phys. Rev. Lett., 77:5417, 1996.

[36] Y. Ando et al. Phys. Rev. Lett., 87:017001, 2001. 
[37] S. Uchida et al. Phys. Rev. B, 43:7942, 1991.

[38] C.C. Tsuei and J.R. Kirtley. Rev. Mod. Phys., 72:969, 2000.

[39] Y.J. Uemura et al. Phys. Rev. Lett., 66:2665, 1991.

[40] A. Damascelli, Z. Hussain, and Z.-X. Shen. Rev. Mod. Phys., 75:473, 2003.

[41] N.E. Hussey et al. Nature, 425:814, 2003.

[42] M. Plate et al. cond-mat/0503117, 2005.

[43] C.G. Olson et al. Science, 245:731, 1989.

[44] T. Takahashi et al. Nature, 334:691, 1988.

[45] B.O. Wells et al. Phys. Rev. B, 46:11830, 1992.

[46] Z.-X. Shen et al. Phys. Rev. Lett., 70:1553, 1993.

[47] W.N. Hardy et al. Phys. Rev. Lett., 70:3999, 1993.

[48] A. G. Loeser et al. Science, 273:325, 1996.

[49] H. Ding et al. Nature, 382:51, 1996.

[50] B.O. Wells et al. Phys. Rev. Lett., 74:964, 1995.

[51] L.H. Tjeng, N.B. Brookes, and B. Sinkovic. J. Electron Spectrosc. Relat. Phenom., 117-118:189, 2001.

[52] J.W. Allen et al. Phys. Rev. Lett., 64:595, 1990.

[53] A. Ino et al. Phys. Rev. Lett., 79:2101, 1997.

[54] Z.-X. Shen et al. Phys. Rev. B, 44:12098, 1991.

[55] N. Harima et al. Phys. Rev. B, 67:172501, 2003.

[56] P.G. Steeneken et al. Phys. Rev. Lett., 90:247005, 2003. 
[57] T. Valla et al. Science, 285:2110, 1999.

[58] P.V. Bogdanov et al. Phys. Rev. Lett., 85:2581, 2000.

[59] A. Lanzara et al. Nature, 412:510, 2001.

[60] C.N. Berglund and W.E. Spicer. Physical Review, 136 (4A):1030, 1964.

[61] G.W. Gobeli, E.O. Kane, and F.G. Allen. Phys. Rev. Lett., 12:94, 1964.

[62] E.O. Kane. Phys. Rev. Lett., 12:97, 1964.

[63] N.V. Smith and L.F. Mattheiss. Phys. Rev. B, 9:1341, 1974.

[64] N.V. Smith. Phys. Rev. B, 9:1365, 1974.

[65] N.V. Smith, M.M. Traum, and F.J. DiSalvo. Solid State Comm., 15:211, 1974.

[66] M.M. Traum, N.V. Smith, and F.J. DiSalvo. Phys. Rev. Lett., 32:1241, 1974.

[67] N.V. Smith and M.M. Traum. Phys. Rev. B, 11:2087, 1975.

[68] F. Reinert et al. Phys. Rev. B, 63:115415, 2001.

[69] T. Kiss et al. Phys. Rev. Lett., 94:057001, 2005.

[70] T. Shimojima et al. Phys. Rev. B, 71:020505, 2005.

[71] S. Tsuda et al. cond-mat/0409219, 2004.

[72] S. Hufner. Photoelectron Spectroscopy : Principles and Applications. SpringerVerlag, 1995.

[73] A. Ino. Photoemission Study of the High-Temperature Superconductor $\mathrm{La}_{2-x} \mathrm{Sr}_{x} \mathrm{CuO} \mathrm{O}_{4}$. PhD thesis, University of Tokyo, 1999.

[74] G.D. Mahan. Phys. Rev. B, 2:4334, 1970.

[75] W.L. Schaich and N.W. Ashcroft. Phys. Rev. B, 3:2452, 1971. 
[76] P.J. Feibelman and D.E. Eastman. Phys. Rev. B, 10:4932, 1974.

[77] A. Bansil and M. Lindroos. Phys. Rev. Lett., 83:5154, 1999.

[78] M. Lindroos, S. Sahrakorpi, and A. Bansil. Phys. Rev. B, 65:054514, 2002.

[79] T. Miller, W.E. McMahon, and T.C. Chiang. Phys. Rev. Lett., 77:1167, 1996.

[80] M.R. Norman et al. Phys. Rev. B, 59:11191, 1999.

[81] M.P. Seah and W.A. Dench. Surf. Interface Anal., 1:2, 1979.

[82] L. Hedin and S. Lundqvist. Solid State Physics : Advances in Research and Applications, volume 23. Academic, New York, 1969.

[83] Kevan S.D. Angle Resolved Photoemission - Theory and Current Applications. Elsevier Science, Amsterdam, 1992.

[84] L. Hedin and J.D. Lee. J. Electron Spectrosc. Relat. Phenom., 124:289, 2002.

[85] M. Randeria and J.C. Campuzano. cond-mat/9709107, 1997.

[86] G.D. Mahan. Many-Particle Physics. Kluwer Academic, 3rd edition, 2000.

[87] M. Randeria et al. Phys. Rev. Lett., 74:4951, 1995.

[88] F. Ronning et al. Science, 282:2067, 1998.

[89] K. Okazaki. Photoemission Studies of Temperature-Induced Metal-Insulator Transitions in Transition-Metal Oxides. PhD thesis, University of Tokyo, 2002.

[90] S. LaShell, E. Jensen, and T. Balasubramanian. Phys. Rev. B, 61:2371, 2000.

[91] M. Hengsberger et al. Phys. Rev. B, 60:10796, 1999.

[92] S. Engelsberg and J.R. Schrieffer. Physical Review, 131:993, 1963.

[93] J.M. Rowell et al. Phys. Rev. Lett., 10:334, 1963.

[94] D.J. Scalapino et al. Physical Review, 148:263, 1966. 
[95] G. Grimvall. The Electron-Phonon Interaction in Metals. North-Holland, New York, 1981.

[96] J. Shi et al. Phys. Rev. Lett., 92:186401, 2004.

[97] X.J. Zhou et al. cond-mat/0405130, 2004.

[98] V.N. Strocov, R. Claessen, and P. Blaha. Phys. Rev. B, 68:144509, 2003.

[99] V.A. Gavrichkov, A.A. Borisov, and S.G. Ovchinnikov. Phys. Solid State, 43:1876, 2001.

[100] A.S. Moskvin, E.N. Kondrashov, and V.I. Cherepanov. Phys. Solid State, 43:823, 2001.

[101] D.S. Dessau et al. Phys. Rev. Lett., 71:2781, 1993.

[102] F. Kohsaka et al. in preparation 2005.

[103] N. Mannella et al. J. Electron Spectrosc. Relat. Phenom., 141:45, 2004.

[104] N.J.C. Ingle et al. in preparation 2005.

[105] T. Fujii and I. Terasaki. Physica C, 392-396:238, 2003.

[106] K. Tanaka et al. in preparation 2005.

[107] K. Tanaka. Photoemission Study of Bi-cuprate high- $T_{c}$ superconductors in the lightly-doped to underdoped regions. PhD thesis, University of Tokyo, 2004.

[108] H. Eisaki et al. Phys. Rev. B, 69:064512, 2004.

[109] D.L. Feng et al. Phys. Rev. Lett., 86:5550, 2001.

[110] Y.D. Chuang et al. Phys. Rev. Lett., 87:117002, 2001.

[111] A.A. Kordyuk et al. Phys. Rev. Lett., 89:077003, 2002.

[112] D. Vaknin et al. Phys. Rev. B, 56:8351, 1997. 
[113] G. Blumberg et al. Phys. Rev. B, 53:R11930, 1996.

[114] Y. Kohsaka et al. J. Am. Chem. Soc, 124:12275, 2002.

[115] L.L. Miller et al. Phys. Rev. B, 41:1921, 1990.

[116] Z. Hiroi, N. Kobayashi, and M. Takano. Nature, 371:139, 1994.

[117] K. Waku et al. Phys. Rev. B, 70:134501, 2004.

[118] A.F. Ioffe and A.R. Regel. Prog. Semicond., 4:237, 1960.

[119] K. Ohishi et al. cond-mat/0412313, 2004.

[120] R. Matzdorf et al. Science, 289:746, 2000.

[121] A. Damascelli et al. Phys. Rev. Lett., 85:5194, 2000.

[122] K.M. Shen et al. Phys. Rev. B, 64:180502(R), 2001.

[123] M.A. VanHove and S.Y. Tong. Surface Crystallography by LEED. SpringerVerlag, Heidelberg, 1979. (http://electron.lbl.gov/leedpack/).

[124] L.F. Mattheiss. Phys. Rev. B, 42:354, 1990.

[125] R. Hayn et al. Phys. Rev. B, 60:645, 1999.

[126] J.J.M. Pothuizen et al. Phys. Rev. Lett., 78:717, 1997.

[127] F. Ronning et al. Phys. Rev. B, 67:165101, 2003.

[128] T. Boske et al. Phys. Rev. B, 56:3438, 1997.

[129] T. Tohyama and S. Maekawa. Supercond. Sci. Technol., 13:R17, 2000.

[130] C. Kim et al. Phys. Rev. B, 65:174516, 2002.

[131] H. Eskes, L.H. Tjeng, and G.A. Sawatzky. Phys. Rev. B, 41:288, 1990.

[132] D.W. Turner. Molecular Photoelectron Spectroscopy. Wiley, New York, 1970. 
[133] R.P. Feynman. Phys. Rev., 97:660, 1955.

[134] T. Holstein. Ann. Phys., 8:325, 1959.

[135] T. Holstein. Ann. Phys., 8:343, 1959.

[136] D. Emin and T. Holstein. Ann. Phys., 53:439, 1969.

[137] B.S. Shastry and D.C. Mattis. Phys. Rev. B, 24:5340, 1981.

[138] A. Auerbach and B.E. Larson. Phys. Rev. Lett., 66:2262, 1991.

[139] S.G. Ovchinnikov et al. J. Phys. Cond. Mat., 16:L93, 2004.

[140] E. Dagotto. Rev. Mod. Phys., 66:763, 1994.

[141] A. S. Mishchenko and N. Nagaosa. Phys. Rev. Lett., 93:036402, 2004.

[142] R. Kilian and G. Khaliullin. Phys. Rev. B, 60:13458, 1999.

[143] J. van der Brink, P. Horsch, and A.M. Oles. Phys. Rev. Lett., 85:5174, 2000.

[144] J. Schafer et al. Phys. Rev. Lett., 92:094205, 2004.

[145] P.H. Citrin, P. Eisenberger, and D.R. Hamann. Phys. Rev. Lett., 33:965, 1974.

[146] P.H. Citrin, P. Eisenberger, and D.R. Hamann. Phys. Rev. B, 15:2923, 1977.

[147] P.H. Citrin, G.K. Wertheim, and Y. Baer. Phys. Rev. B, 16:4256, 1977.

[148] P.H. Citrin, G.K. Wertheim, and Y. Baer. Phys. Rev. B, 35:885, 1975.

[149] D.S. Dessau et al. Phys. Rev. Lett., 81:192, 1998.

[150] Y.D. Chuang et al. Science, 292:1501, 2001.

[151] A. J. Millis, B. I. Shraiman, and R. Mueller. Phys. Rev. Lett., 77:175, 1996.

[152] V. Perebeinos and P.B. Allen. Phys. Rev. Lett., 85:5178, 2000.

[153] K. Okazaki et al. Phys. Rev. B, 69:140506(R), 2004. 
[154] K. Okazaki et al. Phys. Rev. B, 69:165104, 2004.

[155] D. Schrupp et al. cond-mat/0405623, 2004.

[156] L. Perfetti et al. Phys. Rev. Lett., 87:216404, 2001.

[157] L. Perfetti et al. Phys. Rev. B, 66:075107, 2002.

[158] Y. Shibata, T. Tohyama, and S. Maekawa. Phys. Rev. B, 59:1840, 1999.

[159] K. Huang and A. Rhys. Proc. Roy. Soc., Ser. A, 208:352, 1951.

[160] C. Kittel. Quantum Theory of Solids. John Wiley and Sons, 2nd edition, 1987.

[161] J.P. Falck et al. Phys. Rev. Lett., 69:1109, 1992.

[162] C.Y. Chen et al. Phys. Rev. B, 43:392, 1991.

[163] M.C. Schabel et al. Phys. Rev. B, 57:6090, 1998.

[164] A. Koitzsch et al. Phys. Rev. B, 66:024519, 2002.

[165] C.P. Flynn. Phys. Rev. Lett., 37:1445, 1975.

[166] R. Joynt. Science, 284:777, 1999.

[167] K. Schulte et al. Phys. Rev. B, 63:165429, 2001.

[168] F. Moser and F. Urbach. Physical Review, 102:1519, 1956.

[169] T. Hanaguri et al. Nature, 430:1001, 2004.

[170] O. Rosch and O. Gunnarsson. Eur. Phys. J. B, 43:11, 2005.

[171] T. Yoshida et al. Phys. Rev. Lett., 91:027001, 2003.

[172] D.S. Marshall et al. Phys. Rev. Lett., 76:4841, 1996.

[173] A.S. Mishchenko. in preparation 2005.

[174] F. Ronning et al. Phys. Rev. B, 71:094518, 2005. 
[175] C. Dahnken et al. Phys. Rev. B, 70:245110, 2004.

[176] H. Eskes and R. Eder. Phys. Rev. B, 54:R14226, 1996.

[177] C. Grober, R. Eder, and W. Hanke. Phys. Rev. B, 62:4336, 2000.

[178] S. Pairault, D. Senechal, and A.-M. S. Tremblay. Eur. Phys. J. B, 16:85, 2000.

[179] E.C. Carter and A.J. Schofield. Phys. Rev. B, 70:045107, 2004.

[180] H. Eskes and G.A. Sawatzky. Phys. Rev. B, 44:9656, 1991.

[181] B.I. Shraiman and E.D. Siggia. Phys. Rev. Lett., 61:467, 1988.

[182] D.N. Sheng, Y.C. Chen, and Z.Y. Weng. Phys. Rev. Lett., 77:5102, 1996.

[183] T.D. Stanescu and P. Phillips. Phys. Rev. Lett., 91:017002, 2003.

[184] T.D. Stanescu and P. Phillips. Phys. Rev. B, 69:245104, 2004.

[185] P. Phillips, D. Galanakis, and T.D. Stanescu. Phys. Rev. Lett., 93:267004, 2004.

[186] H. Yagi. private communication 2005.

[187] X.J. Zhou et al. Nature, 423:398, 2003.

[188] M. Imada, A. Fujimori, and Y. Tokura. Rev. Mod. Phys., 70:1039, 1998.

[189] A. Georges et al. Rev. Mod. Phys., 68:13, 1996.

[190] A. Georges. cond-mat/0403123, 2004.

[191] T. Pruschke, D.L. Cox, and M. Jarrell. Phys. Rev. B, 47:3553, 1993.

[192] Y. Kohsaka. in preparation 2005.

[193] O. Rosch et al. cond-mat/0504660, 2005.

[194] X.-G. Wen and P.A. Lee. Phys. Rev. Lett., 76:503, 1996.

[195] S. Maekawa et al. Physica C, 152:133, 1988. 
[196] G. Kotliar and J. Liu. Phys. Rev. Lett., 61:1784, 1988.

[197] N. Furukawa, T.M. Rice, and M. Salmhofer. Phys. Rev. Lett., 81:3195, 1998.

[198] N. Furukawa and T.M. Rice. J. Phys. : Condens. Matt., 10:L381, 1998.

[199] C.M. Honerkamp et al. Phys. Rev. B, 63:035109, 2001.

[200] M.L. Salkola, V.J. Emery, and S.A. Kivelson. Phys. Rev. Lett., 77:155, 1996.

[201] R.S. Markiewicz et al. Phys. Rev. B, 62:1252, 2000.

[202] Y. Kohsaka et al. Journal of the Physical Society of Japan, 72:1018, 2003.

[203] K.M. Shen. in preparation 2005.

[204] R.S. Markiewicz et al. cond-mat/0503064, 2005.

[205] Y. Kohsaka et al. Phys. Rev. Lett., 93:097004, 2004.

[206] J.E. Hoffman et al. Science, 297:1148, 2002.

[207] K. McElroy et al. Nature, 422:592, 2003.

[208] M. Vershinin et al. Science, 303:1995, 2004.

[209] C. Howald et al. Phys. Rev. B, 67:014533, 2003.

[210] Y. Aiura et al. Phys. Rev. Lett., 91:256404, 2003.

[211] T.E. Kidd et al. Phys. Rev. Lett., 88:226402, 2002.

[212] R. Liu et al. Phys. Rev. Lett., 80:5762, 1998.

[213] J. Schafer et al. Phys. Rev. Lett., 87:196403, 2001.

[214] Th. Straub et al. Phys. Rev. Lett., 82:4504, 1999.

[215] T.E. Kidd et al. Phys. Rev. Lett., 85:3684, 2000.

[216] S. Hatta et al. Phys. Rev. B, 71:041401(R), 2005. 
[217] T. Nakagawa et al. Phys. Rev. B, 67:241401(R), 2003.

[218] V. Brouet et al. Phys. Rev. Lett., 93:126405, 2004.

[219] D.L. Feng et al. cond-mat/9908056, 1999.

[220] X.J. Zhou et al. Science, 286:268, 1999.

[221] H.C. Fu, J.C. Davis, and D.-H. Lee. cond-mat/0403001, 2004.

[222] E. Fradkin S.E. Brown and S.A. Kivelson. cond-mat/0501654, 2005.

[223] A.M. Gabovich et al. Supercond. Sci. Tech., 14:R1, 2001.

[224] J.E. Hoffman et al. Science, 295:466, 2002.

[225] K. McElroy et al. cond-mat/0404005, 2004.

[226] S. Sachdev. Science, 288:475, 2000.

[227] H.-D. Chen et al. Phys. Rev. Lett., 93:187002, 2004.

[228] G. Gruner. Density Waves in Solids. Addison Wesley Longman, 1st edition, 1994.

[229] M. Civelli et al. cond-mat/0411696, 2004.

[230] T.A. Maier, T. Pruschke, and M. Jarrell. Phys. Rev. B, 66:075102, 2002.

[231] D. Senechal et al. Phys. Rev. Lett., 94:156404, 2005.

[232] X.J. Zhou et al. in preparation 2005.

[233] X.J. Zhou et al. Phys. Rev. Lett., 92:187001, 2004.

[234] Y. Iye. Properties of High Temperature Superconductors, volume 3. World Scientific, Singapore, 1992.

[235] D.G. Hawthorn et al. Phys. Rev. Lett., 90:197004, 2003. 
[236] X.F. Sun et al. Phys. Rev. Lett., 90:117004, 2003.

[237] F. Reinert et al. Phys. Rev. Lett., 85:3930, 2000.

[238] A. Chainani et al. Phys. Rev. Lett., 85:1966, 2000.

[239] J. Mesot et al. Phys. Rev. B, 63:224516, 2001.

[240] J. Mesot et al. Phys. Rev. B, 61:11248, 2000.

[241] A.L. Efros and B.I. Shklovskii. J. Phys. C, 8:L49, 1975.

[242] S.H. Pan et al. Nature, 413:282, 2001.

[243] C.M. Howald, P. Fournier, and A. Kapitulnik. Phys. Rev. B, 64:100504, 2001.

[244] M. Matsuda et al. Phys. Rev. B, 65:134515, 2002.

[245] E. Cuevas, M. Ortuno, and J. Ruiz. Phys. Rev. Lett., 71:1871, 1993.

[246] D.J. Huang, G. Reisfeld, and M. Strongin. Phys. Rev. B, 55:R1977, 1997.

[247] P. Starowicz et al. Phys. Rev. Lett., 89:256402, 2002.

[248] H. Hovel et al. Phys. Rev. Lett., 81:4608, 1998.

[249] B.O. Wells et al. Science, 277:1067, 1997.

[250] K. Yamada et al. Phys. Rev. B, 57:6165, 1998.

[251] T. R. Thurston et al. Phys. Rev. B, 40:4585, 1989.

[252] N.P. Armitage et al. Phys. Rev. Lett., 68:064517, 2003.

[253] F. Ronning et al. Phys. Rev. B, 67:035113, 2003.

[254] D.L. Feng et al. Phys. Rev. B, 65:220501(R), 2002.

[255] P.V. Bogdanov et al. Phys. Rev. B, 64:180505(R), 2001.

[256] M.C. Asensio et al. Phys. Rev. B, 67:014519, 2003. 
[257] S. Massidda, J.J. Yu, and A.J. Freeman. Physica C, 152:251, 1988.

[258] K.M. Shen et al. Phys. Rev. Lett., 93:267002, 2004.

[259] J.J. Yeh and I. Lindau. At. Data Nucl. Data Tables, 32:1, 1985.

[260] Y. Maeno et al. Nature, 372:532, 1994.

[261] A.P. Mackenzie et al. Phys. Rev. Lett., 76:3786, 1996.

[262] K. Ishida et al. Nature, 396:658, 1998.

[263] I.I. Mazin and D.J. Singh. Phys. Rev. Lett., 82:4324, 1999.

[264] T. Imai et al. Phys. Rev. Lett., 81:3006, 1998.

[265] Y. Sidis et al. Phys. Rev. Lett., 83:3320, 1999.

[266] P.K. de Boer and R.A. de Groot. Phys. Rev. B, 59:9894, 1999.

[267] A. Morpurgo. private communication 2001.

[268] T. Oguchi. Phys. Rev. B, 51:1385, 1995.

[269] D.J. Singh. Phys. Rev. B, 52:1358, 1995.

[270] T. Yokoya et al. Phys. Rev. B, 54:13311, 1996.

[271] D.H. Lu et al. Phys. Rev. Lett., 76:4845, 1996.

[272] Z. Fang. private communication 2001.

[273] A. Liebsch and A. Lichtenstein. Phys. Rev. Lett., 84:1591, 2000.

[274] A. Liebsch. private communication 2001.

[275] C. Buzea and T. Yamashita. Supercond. Sci. Technol., 14:R115, 2001.

[276] S.L. Budko et al. Phys. Rev. Lett., 86:1877, 2001.

[277] D.G. Hinks, H. Claus, and J.D. Jorgensen. Nature, 411:457, 2001. 
[278] W.L. MacMillan. Phys. Rev., 167:331, 1968.

[279] P.B. Allen and R.C. Dynes. Phys. Rev. B, 12:905, 1975.

[280] J.E. Hirsch. Phys. Lett. A, 282:392, 2001.

[281] M. Imada. J. Phys. Soc. Jpn., 70:1218, 2001.

[282] N. Furukawa. J. Phys. Soc. Jpn., 70:1483, 2001.

[283] N.F. Mott. Metal-Insulator Transitions. Taylor and Francis, London, 1990.

[284] S. Lee et al. J. Phys. Soc. Jpn., 70:2255, 2001.

[285] J. Kortus et al. Phys. Rev. Lett., 86:4656, 2001.

[286] J.M. An and W.E. Pickett. Phys. Rev. Lett., 86:4366, 2001.

[287] V.M. Silkin, E.V. Chulkov, and P.M. Echenique. Phys. Rev. B, 64:172512, 2001.

[288] Z. Li et al. Phys. Rev. B, 65:100507, 2002.

[289] V.D.P. Servedio, S.-L. Drechsler, and T. Mishonov. cond-mat/0111434, 2001.

[290] A.R. Law, M.T. Johnson, and H.P. Hughes. Phys. Rev. B, 34:4289, 1986.

[291] G. Rubio-Bollinger, H. Suderow, and S. Vieira. Phys. Rev. Lett., 86:5582, 2001.

[292] G. Karapetrov et al. Phys. Rev. Lett., 86:5582, 2001.

[293] F. Giubileo et al. Phys. Rev. Lett., 87:177008, 2001.

[294] P. Szabo et al. Phys. Rev. Lett., 87:137005, 2001.

[295] T. Takahashi et al. Phys. Rev. Lett., 86:4915, 2001.

[296] S. Tsuda et al. Phys. Rev. Lett., 87:177006, 2001.

[297] S.D. Kevan and E. Rotenberg. J. Electron Spectrosc. Relat. Phenom., 117118:57, 2001.

[298] R. Osborn et al. Phys. Rev. Lett., 87:17005, 2001. 